\title{
Complementary Inferences on Theoretical Physics and Mathematics
}

\author{
Mesut KAVAK
}

\begin{abstract}
I have been working for a long time about basic laws which direct existence [1], and for some mathematical problems which are waited for a solution. I can count myself lucky, that I could make some important inferences during this time, and I published them in a few papers partially as some propositions. This work aimed to explain and discuss these inferences all together by relating them one another by some extra additions, corrections and explanations being physical phenomena are prior. There are many motivation instruments for exact physical inferences.
\end{abstract}

\section{Introduction}

It seems, that existence cannot be separated from each other because of an absolute entanglement. Even if it had already happened or is going to happen in future, each influence which exists within possibility border, already had been existed in imaginary time which is an extremely interesting time concept. Absolute space which has infinite dimension and volume, and emergence area which appears over the absolute space, were always together but a special condition in the imaginary time. This appearance emerges as a dimensional drop and an acceleration decrease from infinite value by using from the absolute space as a part of it. Each small point of the emergence area which is the result of this drop gets different emergence time priority even for any short time interval of any short time interval as it has to exist as a virtual part of the absolute space. The emergence in this way renders impossible to exist of emergence area or emergence area objects constantly without inner or outer space displacement, and to move in a linear direction. Right this point, infinite number of virtual small forces which emerge as a result of the infinite time differences and make matter move to emerge of motion, render impossible to apply force linearly and without dimension; hence the emerging force is an area force as a distributed force, and matter is its result as an uncertain matter which all measurable values of it change over time.

As matter is virtual part of absolute space, uncertainty level increases or decreases between absolute absence and absoluteness. Because of the emerging uncertainty [2], matter is only able to emerge by some periods. This phenomenon renders matter illusion, and also renders it pretty process-able as there is no alternative. Uncertain matter can only be formed over absolute space as waves by forcing each second and actually even in each small period of time as well. It gains energy equal to the work done of this forcing against tendency to surrender of the absolute space; but as the work done is not done untimely manner, matter does not gain infinite work potential. These conditions cause that matter has been emerging by infinite number of side effects on itself, and you cannot know that this is an order or is chaos.

\section{Theoretical Analysis of Motion}

Motion is change in position of an object over time; so to emerge of a motion certainly; distance, velocity, time and time-driven inertia namely latency have to emerge all together without any priority. As it can get a scalar value for its all elements which create it out, also it can be defined as vector quantity if there is a reference because of emerging directions, and magnitude of motion is not independent of magnitude of its elements.

\subsection{The Geometric Rules of Physical Medium}

There exist many exact proofs about the rules of right triangle in abstract math; but what are the actual geometry and mechanism in real physical medium and for real math? What an undertaking can give a triangle about existence to understand it? Actually, it is able to explain everything.

\subsubsection{X-Ray Images of the Secret Geometry}

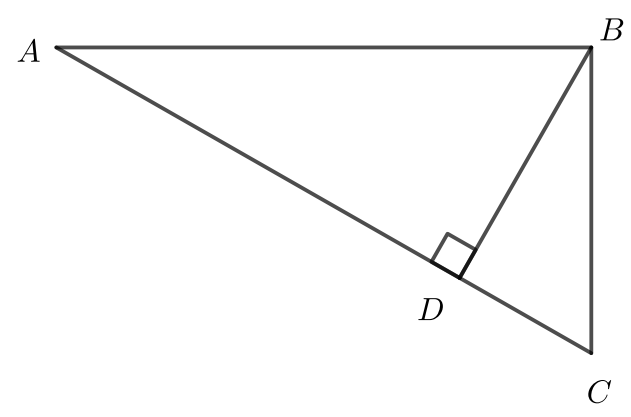

Fig. 1: This is an Euclidean right triangle. Euclidean geometry is the geometry which areas, angles and lengths are related as equality together with whole and certain numbers and there are no complex, uncertain or irrational numbers, irrational numbers are with a limit. Instead of this right triangle, it can be used a triangle which there is no right angle occurs in it. The shape is random. It does not mean actual geometry. For example, BD length can be longer than AD length in the below stated calculations.

There is a representation of an Euclidean geometry. In this case, being $\mathrm{BD}$ and $\mathrm{BC}$ are fixed, assume that $\mathrm{AB}$ is lengthened to any other $\mathrm{AB}_{2}$ length. Here $A$ point can be assumed as moving body in free space, $\mathrm{B}$ point is a fixed point which its coordinate is known in space and $\mathrm{C}$ is an observer.

For the lengthened hypotenuse on Fig. 1, over the inequality and definition of $\mathrm{AB}_{2}>\mathrm{AB}$, it becomes (1) over the inequality of $\mathrm{BD}_{2}^{2}-\mathrm{BD}^{2}>\mathrm{AD}^{2}-\mathrm{AD}_{2}^{2}$,

$$
1>\frac{\mathrm{AD}^{2}-\mathrm{AD}_{2}^{2}}{\mathrm{BD}_{2}^{2}-\mathrm{BD}^{2}}
$$

where $\mathrm{BD}^{2}+\mathrm{AD}^{2}=\mathrm{AB}^{2}$ and $\mathrm{BD}_{2}^{2}+\mathrm{AD}_{2}^{2}=\mathrm{AB}_{2}^{2}$ are the equations over Pythagorean theorem. In the same manner, it 
becomes (2),

$$
\mathrm{BD}^{2}+\mathrm{DC}^{2}=\mathrm{BD}_{2}^{2}+\mathrm{DC}_{2}^{2}
$$

where $\mathrm{h}^{2}+\mathrm{t}^{2}=\mathrm{y}^{2}$ and $\mathrm{h}_{2}^{2}+\mathrm{t}_{2}^{2}=\mathrm{y}^{2}$ are the equations over Pythagorean theorem. If (2) is edited, it becomes $\mathrm{DC}^{2}-$ $\mathrm{DC}_{2}^{2}=\mathrm{BD}_{2}^{2}-\mathrm{BD}^{2}$; thus if $\mathrm{DC}^{2}-\mathrm{DC}_{2}^{2}$ is used instead of $\mathrm{BD}_{2}^{2}-\mathrm{BD}^{2}$ on (1), also it becomes (3) over $\mathrm{DC}^{2}-\mathrm{DC}_{2}^{2}>$ $\mathrm{AD}^{2}-\mathrm{AD}_{2}^{2}$ inequality.

$$
1>\frac{\mathrm{AD}^{2}-\mathrm{AD}_{2}^{2}}{\mathrm{DC}^{2}-\mathrm{DC}_{2}^{2}}
$$

Now the actual displacement inequalities have been determined. Right this point, assume that there is no displacement, namely there is no lengthening. For this condition, it becomes $\mathrm{AB}_{2}=0, \mathrm{DC}_{2}=0$ and $\mathrm{AD}_{2}=0$; thus (3) becomes (4),

$$
\mathrm{DC}^{2}>\mathrm{AD}^{2}
$$

and (1) becomes (5).

$$
-\mathrm{BD}^{2}>\mathrm{AD}^{2}
$$

The inequality of (5) actually means it is impossible to be $\mathrm{AD}=\mathrm{DC}$ that means perpendicularity is impossible in universe. This also means that at the same time no lengths can be the same. From physical perspective, it means each point of free space has the same speed and energy magnitude at the end of 1 second but the same time, and emergence is one by one for the total of universe. Each point emerges by order. This also means, for any force-applied, since there is no middle point the natural motion is always circular as there is no alternative. Because of circular displacement, centrifugal force is always together with motion.

The inequality of (5) has another results, and are as the below stated ones.

- Exactly there is no middle point place for any size part of area; so a motion is always circular for any forceapplied.

- A right angle cannot emerge. It is only close to right angle due to the energy which area holds.

- It cannot be drawn two line segments which have the same length from a point in space to other two points. Namely, 3 or more objects cannot take place in space being the distance between each of them is the same. There is a time difference between each point of space at the same assumed global time.

- For $\mathrm{AD} \neq \mathrm{DC}$, it also becomes $\mathrm{AB} \neq \mathrm{BC}, \mathrm{BD} \neq \mathrm{DC}$, $\mathrm{BD} \neq \mathrm{AD}, \mathrm{BD} \neq \mathrm{AB}$ and more. Namely, while $\mathrm{AB}$ is lengthened, $\mathrm{BC}$ or the other lengths cannot protect own actual length. The medium is conservative. This also means, that length and thus 1 dimension do not exist alone; because 2 objects cannot take place at a distance relatively to each other. Namely, length is not absolute since is relative. Length occurs in a limited time interval and gets lost constantly, and cannot be independent on speed. Higher dimension parts of lower dimensions have different size than lower dimensional parts. Namely a 2 dimensional square cannot be used by the same size to create a 3 dimensional cube.

- The shortest distance between 2 objects is not a line segment. This distance is an arc so close to a line segment.

- Parallel two line segment cannot be drawn beyond drawing line segment. They are exactly intersected, and the intersection point occurs according to area multitude of conservative area. By this way, no object can protect its speed. There is always an opposite space force to decelerate. By this way, as the same is acceptable for the existent smallest infinite part as well, constant speed motion or constant acceleration motion is not possible. Motion emerges by parts, and is always with small acceleration parts has frequency changing over time, and the result is average as assumed constant speed, and is seen as constant speed.

- A closed curve is not possible. Only infinite space closes curve. Limited space is not closed but is conservative.

- Motion cannot be independent of medium whatever the distance between moving bodies. This means, that there is an absolute entanglement between area and lengths in area although there is time differences. It shows one-piece behavior. This is only possible with emergence one by one. Namely, all mass is a single mass with emergence priority. Each point occurs by order. In this way, information is conserved forever; because flexible collision is not possible. There never occurs a mutual extinction which is because of decrease in the energy and mass by certain, whole numbers. Instead of it, during collisions, energy and mass values get smaller to infinite small part as irrational numbers; but it never disappears.

- There is always an emerging centrifugal force for motion because of circular displacement. In 3D, this may cause an outer space motion because of

$$
\mathrm{F}=\frac{\mathrm{mv}^{2} \sin (\alpha)}{\mathrm{r}}
$$

This motion can emerge because of an energy reduction which causes imbalance in motion which is the source of current total energy of objects.

- No object can be static. They always experience a potential difference since there is time difference and thus different densities over time, and move in the direction of lower density, more ordered area from disordered area. Also they always move in the directions of the one by one emergence since to be one of them before or after of the same two Ft work for the same force and time values as $+\mathrm{Ft}$ and $-\mathrm{Ft}$ changes displacement even for conservative energy and momentum.

- Over the law of induction, because of the time differences since any value of matter is different in each time interval during emergence constantly, the equation of

$$
\varepsilon=\mathrm{N}_{2}-\mathrm{N}_{1} \frac{\Phi_{2}-\Phi 1}{\mathrm{t}_{2}-\mathrm{t}_{1}}
$$

shows, that it is always $\varepsilon>0$ where $\varepsilon \in \mathbb{R}$. It means, that a coil even placed at infinite distance from a magnetic field has a voltage difference between the two top of the wire. If there is a fixed matter in the core, voltage will increase. If there is a fixed magnet in the core, then even if also the coil is fixed, the voltage is going to greatly increase; so it means, that even by switching two fixed coils have permanent magnet core and have different resistances will cause magnetic flux change; because electrons' orbital will slide even a current will not emerge, that it can emerge by required assembly and switching repeat. Maybe we can create a micro generator like producing a CPU that sheets between two permanent magnets will be connected serially to create high voltage difference to create electrical current beyond sliding orbitals. Free energy and thus self-running 
devices are possible since total energy of the medium is conserved. This is a focus, and you make matter worked by its own total energy. You must use more material. Namely, the same amount material generate more electricity by classical methods which require an external energy resource.

\subsubsection{Progressive Image}

For the lengthened hypotenuse of Fig. 1, over the inequality of $\left(\mathrm{AD}_{2}+\mathrm{DC}_{2}\right)^{2}>(\mathrm{AD}+\mathrm{DC})^{2}$ where $\mathrm{AB}_{2}^{2}+\mathrm{BC}_{2}^{2}=$ $\left(\mathrm{AD}_{2}+\mathrm{DC}_{2}\right)^{2}$ and $\mathrm{AB}^{2}+\mathrm{BC}^{2}=(\mathrm{AD}+\mathrm{DC})^{2}$, it becomes (6) for $\mathrm{AB}_{2}=0$ that means no lengthening.

$$
0>(\mathrm{AD}+\mathrm{DC})^{2}
$$

It means, that $\mathrm{AD}+\mathrm{DC} \notin \mathbb{R}$. If you assume, that $\mathrm{AD}+$ $\mathrm{DC}=0$, it becomes $0>0$; thus actually non of them can be 0 . It means, that even if there would be no lengthening and thus no motion, there were already area and motion. They are deterministic and cannot be $0 . \mathrm{AD}+\mathrm{DC}$ is always an imaginary number for the condition of (6).

In accordance with conservative space, over the components of the right triangle, it becomes $\mathrm{AB}+\mathrm{BC}+\mathrm{m}=\mathrm{n}$ and $\mathrm{AB}^{2}+\mathrm{BC}^{2}=\mathrm{m}^{2}$ where $\mathrm{m}=\mathrm{AD}+\mathrm{DC}$; thus becomes $\tau+\mathrm{DC}+\mathrm{m}=\mathrm{n}$ and $\tau^{2}+\mathrm{t}^{2}=\mathrm{m}^{2}$ where $\tau$ and $\mathrm{t}$ are time here, and is $\mathrm{m}=\sqrt{\tau^{2}+\mathrm{t}^{2}}$. It seems, that $\mathrm{AB}$ and $\mathrm{BC}$ or $\tau$ and t cannot take random values, that they take certain values in accordance with a rule. If $\tau+\mathrm{t}+\mathrm{m}=\mathrm{n}$ is edited, it becomes $(\tau+\mathrm{t})^{2}=(\mathrm{n}-\mathrm{m})^{2}$ and thus it becomes $\tau^{2}+\mathrm{t}^{2}+2 \tau \mathrm{t}=\mathrm{n}^{2}-2 \mathrm{~nm}+\mathrm{m}^{2}$. Since it is $\tau^{2}+\mathrm{t}^{2}=\mathrm{m}^{2}$, finally it becomes (7),

$$
2(\tau \mathrm{t}-\mathrm{nm})-\mathrm{n}^{2}=0
$$

where $\mathrm{n}=\tau+\mathrm{t}+\sqrt{\tau^{2}+\mathrm{t}^{2}}$. For this equation of (7), the roots become (8) as imaginary time since it cannot be 0 over (6).

$$
\tau=\mp i t
$$

As constant speed and so $\mathrm{x}=\mathrm{vt}$ is not possible, because of $\mathrm{x}=\mathrm{at}^{2}$, when real time is emerged, the distance and time emerge in opposite ways over the time on $\mathrm{x}=\mathrm{at}^{2}$, and know, that image appears as illusion in 3D because of the complex roots and thus complex plane which is perpendicular to $(\mathrm{x}, \mathrm{y})$ plane. After that, if you want to express the motion as average, you do not have to use imaginary time since is real time after this, just use its multitude on $\mathrm{x}=\mathrm{vt}$ and it becomes $\mathrm{x}=-\mathrm{vt}$. Riemann Surfaces are the exact medicine for this disease.

Assume, that $\mathrm{AB}=\mathrm{x}$ is lengthened by $\mathrm{k}$, and then also $\mathrm{DC}=\mathrm{t}$ is lengthened automatically because of changing geometry on Fig. 1. For this condition, as is $x \neq t$, also it becomes (9),

$$
\frac{\mathrm{n}_{2}-\mathrm{n}_{1}}{\mathrm{x}} \neq \frac{\mathrm{n}_{2}-\mathrm{n}_{1}}{\mathrm{t}}
$$

for two consecutive increase in $\mathrm{k}$, where $\frac{\mathrm{x}+\mathrm{k}}{\mathrm{x}}, \frac{\mathrm{t}+\mathrm{k}}{\mathrm{t}}, \mathrm{x}_{2}=\mathrm{x}+\mathrm{k}$ and $\mathrm{t}_{2}=\mathrm{t}+\mathrm{k}$. Namely, suck an increase is not possible. If $\mathrm{x}$ changes by a number like $\mathrm{k}$, then $\mathrm{t}$ is going to change by different number. It does not happen in the same ratio. Namely, it accelerates in a conservative area. As the exact opposite is also possible, constant speed motion is not possible. Namely, while you are assuming $\mathrm{x}$ is lengthened by constant speed, since $t$ is going to accelerate, for an assumed small time interval, also you can assume that I did not want to move x, I just wanted to move $t$, and it accelerated. By this acceleration amount, you will see that also $\mathrm{x}$ is accelerated. Namely, these happen at the same time, by acceleration. One element of area cannot be independent of another. namely, if you say $\mathrm{x}$ moved at constant speed, this is only assumption and is an average speed to calculate.

According to these information, it can be said

- There is a single work done to create all the area because of the entanglement and single mass; so each part of space has the same speed as average. None of them can go faster than another one. No point could gain infinite energy because of the time differences, since the work done is not infinite, is done over time, is done at a frequency, as the work done is equal to kinetic energy.

- There is always a space tension since points experience potential difference which is because of emerging different densities over time. Points want to move towards lower density; so also matter is incompressible. It cannot be compressed down to infinite small part. Speed of the work done and frequency of emerging density difference determines this base compressing limit. More energy is required to create denser and thus more disordered area. We can explain it such, that in accordance with conservative area, over the triangle, for a function of $\mathrm{f}(\mathrm{x})=\mathrm{ax}$, it becomes (10) over (10a) where $\mathrm{x}_{2}=\mathrm{x} \mp \mathrm{k}$

$$
\begin{gathered}
\mathrm{x}=\frac{\mp \mathrm{k}}{\sqrt{2}-1} \\
\int \mathrm{axdx}=\int_{\mathrm{x}}^{\mathrm{x}_{2}} \mathrm{ax} \mathrm{dx}
\end{gathered}
$$

Namely, the increase does not happen randomly. You need more speed of the work done to create the area and so need taller $\mathrm{x}$. This creates a space tension always for both compressing and lengthening. There is going to be always a resistance and a limit for the both.

Here, important thing is to change the geometry of the triangle into another shape being the area is the same; so even if this calculation is going to create a trapezoid and a triangle in a wider triangle, both of them are going to have the same area being $\mathrm{x}$ is a constant. There is no difference.

\section{Warning}

- Over (10), it can be said, that $\mathrm{x}$ is always irrational. This is pretty suitable since there are always potential and time differences even for any short interval of any short interval. If also matter could not gain infinite energy and area because of the finite work done, it means, finite matter is virtual part of infinite or infinite information.

All infinite information is certain only. This irrationality cannot happen at the same time. Namely an irrational number cannot be inclusive of works with its all infinite digits after the comma; but its limit may constantly change. Any existent point at different number after comma by increasing, decreasing and getting lost numbers over time. Matter appears at this times. This creates time and thus motion again and again, and also you cannot say is constant speed since is uncertain. The below stated sentences and words like infinite frequency, infinite small mass part or uncertainty etc. must be understood in this way. These mean for example saying like has the smallest mass magnitude. Already otherwise matter could not appear. 
Warning

\subsubsection{The Result}

In accordance with these information, as a result, matter is deterministic. Also it is uncertain as its any measurable value is also uncertain. It has no infinite energy and thus volume. It emerges at a frequency. Right this point, to calculate, there emerge some local and global min. and max. points and threshold values as a reference. Namely, if you make a calculation for centrifugal force over circular displacement, you cannot say 'Alas! The force became infinite, hide behind a stone!' since $\tan (\alpha)$ became 90 according to a reference. It does not happen such. Angle is always fixed even if is irrational; but the same assumed small motion as a reference and threshold value repeats. There is no infinite mass or standing time in black holes or somewhere else in the universe.

For example, since kinetic energy is equal to work done, a stopper opposite limited work on the infinite brings matter total energy out at the amount of this opposite enforcement. Since matter always experiences potential differences, it always moves to lower density and thus more ordered place, and then it again experiences a denser space. This always repeats itself as long as there is a motion and area. Since to be one of them before or after of the same two opposite Ft works in space as $+\mathrm{Ft}$ and $-\mathrm{Ft}$ changes the displacement even momentum and energy will be conserved at that time, if matter is formed by a circular motion, there must be always a potential difference or initial movement for the resultants on Fig. 2 to emerge of motion if you assume the motion closes a perfect circle, that actually does not close, that is not important since the basic logic is going to be the same.

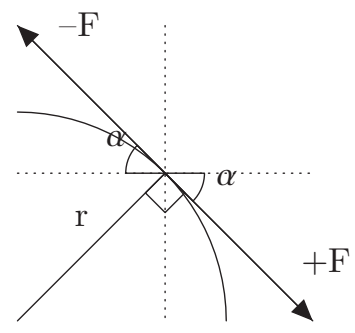

Fig. 2: The resultants

Any way, according to the above stated information, we must leave one of the resultants like on Fig. 2 to emerge of matter to calculate it. It will be enough for the calculation. Being $\mathrm{mvr} / \mathrm{r}=\tan (\alpha)$, for $\operatorname{mvr} / \mathrm{r}=\int \tan (\alpha)$ equation it becomes Eq. (11) for 1 second.

$$
\log _{\mathrm{e}} \cos \alpha=-\mathrm{mv}
$$

As required by uncertainty and one by one emergence, mass decreases at the same ratio with time [1] for the smaller slices of time directly proportional; because wave packet or in the other name assumed existent smallest part of matter repeats itself; but the smallest part may emerge in different ratios with time; so for $\cos ^{-1}\left(\mathrm{e}^{-\mathrm{mvt}}\right)=\alpha$ by decreased mass, $\frac{2 \pi \mathrm{r} \alpha}{360}=\mathrm{vt}$ and $\mathrm{h} v=\mathrm{mv}^{2}$ equations, Eq. (11) turns into Eq. (12) as the below,

$$
\log _{\mathrm{e}} \cos \alpha=-\frac{\pi \mathrm{r} \alpha \mathrm{m}}{180}
$$

where $\mathrm{v}$ is any multitude formation speed as light speed, and it can only be solved by solving equation as an irrational value, as actually this irrationality is required by uncertainty as well.
This angle on Eq. (12) is the angle emerges in the smallest time as required by uncertainty as a threshold value. For this angle, the smallest time in the other name the Planck Time can be found out over $\frac{2 \pi \mathrm{r} \alpha}{360}=\mathrm{vt}$ and the smallest distance can be found out over $\frac{2 \pi \mathrm{r} \alpha}{360}$ as the Planck Length. As you can see, the angle changes according to the multitude of Planck constant but being zero or 90 .

Or for example, if you leave a handful of electrons to a nucleus, it only choose some of them, and the electrons only get certain orbitals or actually get some orbitals they exist more. They cannot exist there randomly. In the same manner, galaxy spiral arms do not take place accidentally. If you place some marbles which have the same mass and density in space being the distance between them is the same, you will see, that they do not come together at a point. Instead of it, according to emerging way which is because of the time differences, they are grouped; but maybe according to emergence way or in the other name spin of the universe, some of them are grouped slower. At the end, again each group create a second group. This continues until coming together. Namely as a result, like in the double slit experiment, whatever the frequency or type of the rays, if light passes through such a slit, the image appears at the screen being some areas are denser even if the light will touch the other points between the denser lines as well.

Actually in topological perspective, there is no difference between a torus and a sphere since a closed curve is not possible. Actually, it means, that there is no difference between shapes. This means, relativistic topology must emerge somewhere or you cannot define shape.

\section{Warning}

\subsection{Theoretic analysis of functions of time}

Velocity is the rate of change of position with respect to a frame of reference. As it emerges in a direction in the space which has another reference, it is defined as vector quantity. It can be demonstrated as Eq. (13) in the simple form as an expression,

$$
\mathbf{v}_{\mathbf{f}}=\mathbf{v}_{\mathbf{t}} \mathrm{t}
$$

where $\mathbf{v}_{\mathbf{f}}, \mathbf{v}_{\mathbf{t}}: \mathbb{R}^{\mathrm{n}} \rightarrow \mathbb{R}^{\mathrm{n}}, \mathbf{a}=\mathbf{v}_{\mathbf{t}}$ as changing velocity over time which means acceleration, where $\mathbf{v}_{\mathbf{f}}=\mathbf{v}$. It gets place in destination equation according to time as Eq. (13a)

$$
\mathbf{x}_{\mathbf{f}}=\mathbf{v}_{\mathbf{f}} \mathbf{t}
$$

where $\mathbf{x}_{\mathbf{f}}=\mathbf{x}$ as distance taken during motion.

Even if there are some minuses according to me, I must use Hilbert Space as there is no alternative yet; so the rule of the below stated vector fields are determined by Hilbert Space. If you detect some contradiction with the fiction of the paper, do not pay attention.

\section{Warning}

Velocity is not independent of distance taken during motion, of time which passes during motion, of energy which causes and keeps motion up during course duration, of inertia which is a result of time as a latency as no motion is able to emerge without time, as also they cannot be independent of one another and velocity. They have to emerge all together without any priority; because they are natural results for each other.

Magnitude of velocity is not independent of magnitude of its elements which emerge all together with them, and magnitude of velocity vector is called as speed which is independent of direction as a scalar value. It can be demonstrated as 
Eq. (14)

$$
\mathrm{v}_{\mathrm{f}}=\left\|\mathbf{v}_{\mathbf{t}}\right\| \mathrm{t}
$$

where $\mathrm{v}_{\mathrm{f}}: \mathbb{R}^{\mathrm{n}} \rightarrow \mathbb{R}$.

\subsubsection{Work done interval}

Let an object which is doing constant speed movement to be accelerated when it had not gotten observational velocity in space before doing constant speed movement. For this condition, during the acceleration work, when the speed reached was too close to the aimed constant speed, the last part of the distance taken would be as Eq. (15),

$$
\mathrm{x}_{\mathrm{p}}=\mathrm{v}_{0} \mathrm{t}+\mathrm{at} \mathrm{t}^{2}
$$

as partial distance taken, of course if you assume that, the initial velocity does a constant speed movement without acceleration during this infinite small part. For Eq. (15a),

$$
\mathrm{v}_{0}=\lim _{\mathrm{t} \rightarrow 0} \frac{\mathrm{x}_{\mathrm{p}}-\mathrm{at} \mathrm{t}^{2}}{\mathrm{t}}
$$

as time must be the smallest time, it means that either $\mathrm{v}_{0}$ had already been absolute as a constant speed motion that means was not created and was without acceleration, or it means the distance taken had already been zero. None of them is possible; thus it means that constant speed motion and constant acceleration motion are not possible, and an object which is doing an assumed constant speed motion in space either accelerates or decelerates even if the amount is too small.

As it will require to be absolute, constant speed motion and constant acceleration motion are not possible. An object which is doing an assumed constant speed movement as dependent manner on time in space, either accelerates or decelerates even if the amount is too small.

\section{Inference: Bad boys}

The famous destination equation which is Eq. (16)

$$
\mathrm{x}(\mathrm{t})=\mathrm{v}_{0} \mathrm{t}+\frac{\mathrm{at}}{2}
$$

is pretty wrong. It is derived over Eq. (16a),

$$
\mathrm{x}(\mathrm{t})=\int\left(\mathrm{v}_{0}+\mathrm{at}\right) \mathrm{d}_{\mathrm{t}}
$$

and is wrong; because if $\mathrm{v}_{0}$ is able to be integrated like $\mathrm{v}_{0} \int \mathrm{d}_{\mathrm{t}}=\mathrm{v}_{0} \mathrm{t}$ as the same as on Eq. (16a), also as the equation is at $=\mathrm{v}$, then $\mathrm{v}$ can be integrated in the same manner. Also for $\mathrm{a}=\mathrm{x} / \mathrm{t}^{2}$, the equation will turn into $\int\left(\mathrm{v}_{0}+(\mathrm{x} / \mathrm{t})\right) \mathrm{d}_{\mathrm{t}}$, and thus turns into Eq. (16b).

$$
\mathrm{x}(\mathrm{t})=\mathrm{v}_{0} \mathrm{t}+\mathrm{x} \ln (\mathrm{t})
$$

These give different results. It is like finding out $\int \mathrm{f}(\mathrm{x}) \mathrm{x} \mathrm{d}_{\mathrm{x}}$, and already integration does not mean sum always. The main expectation for the sum must be $\int \mathrm{dn}$. These are not able to be integrated but specific conditions which have no formulaic qualify as they are only valid for some certain values.

Matter has emergence priority. Each point emerges in order. Each point mass decelerates from an infinite value down to an infinite small value by converging; but as matter has many these small point masses since it emerges along
1 second, actually mass increases over time even if each point mass decreases. Even if constant speed seems not possible, as each point mass in the other name infinite small part of matter has a threshold time value, you must count speed as fixed for this interval as average; so you can state that constant speed motion is possible after this to calculate.

\section{Warning}

As constant speed motion is not possible as absolute without to be created, any work that created constant speed motion is included as well, can only be done in a time interval at a frequency. As there must be infinite frequency, whatever the actual function is, the smallest $\mathrm{f}(\mathrm{x})$ part gains infinite sub parts, and the repeating thing is this the same magnitude value. As a result of this, functions of time as $\mathrm{x}(\mathrm{t})=\mathrm{at}^{2}, \mathrm{v}(\mathrm{t})=\mathrm{at}$, $\mathrm{x}(\mathrm{t})=\mathrm{vt}$ and $\mathrm{v}^{2}=$ ax get these simple forms. Even if observational outer space motion is done one by one, by different time intervals or one-piece without hand taking, there is no difference between them; because already the work is done one by one.

The formulas are not created specifically. There is only 1 math and 1 physics for existence. Even if a universe is a separate existence, all infinite information is one-piece since created together in imaginary time at the same time by the same rule even if a part has been visible in real time; because only density changes; so when I say infinite frequency here, it can be used for any information created or not yet. There is a difference with imaginary time, that in real time, the functions of time remain as catalytic. Namely, they are only for our understanding of relativistic energy and mass formulas, and then they leave. They do not work for real time as also they cannot be integrated since they do not interpret change in time or mass of existence not time or mass change in motion namely in displacement. If you change all existent areas by multiplying by 2 , you changed nothing; but if you change only area of 1 thing, you must proof where did you take it; even so for low speeds, as the difference is going to be a little, we use that classic mechanic formulas as subterfuge; but this should not disrupt our understandings. For an object has v constant speed in space, if you ask the distance taken for a $t$ time you use $d=v t$; but if you draw a graph being $t_{0}=0$ which is the time when you ask and if state that for $\mathrm{x}=\mathrm{t}$ the distance taken is the area between $x$ axis and $f(t)$ function since for each small time, a perpendicular line emerges between $f(t)$ function and $x$ axis namely $t$ axis and thus the sum of the lines is an area, you are going to be wrong; because matter is not continuous in each small time. For your assumption, you accepted the limited area is divided by infinite parts and thus times. You calculated the motions which matter did not experience. The graph already shows you for each time how many distance had the object taken on $y=f(t)$ axis. The classic formulas are counted as integration of many derivatives or the sum of each integration of a derivative. Namely nothing changes. Right this point you can ask like also we divide the limited area for integration of for example formulas of changing masses even if also the work is not done at that times, I can say that it is not the same thing; because by this way the process becomes 
convergence, and the result is still uncertain.

Warning

For the functions of time over $\mathrm{f}^{\prime}\left(\mathrm{t}_{0}\right)=\frac{\mathrm{f}(\mathrm{t})-\mathrm{f}\left(\mathrm{t}_{0}\right)}{\mathrm{t}-\mathrm{t}_{0}}$ by using derivative at a certain point as required by sum of infinite the same work parts, velocity will be as Eq. (17),

$$
\text { at }=\lim _{\mathrm{t}_{0} \rightarrow 0} \frac{\mathrm{at} \mathrm{t}^{2}-\mathrm{at} \mathrm{t}_{0}^{2}}{\mathrm{t}-\mathrm{t}_{0}}
$$

where $f(x)=a x^{2}$. Also it will be as Eq. (18),

$$
\mathrm{v}=\lim _{\mathrm{t}_{0} \rightarrow 0} \frac{\mathrm{vt}-\mathrm{vt} 0}{\mathrm{t}-\mathrm{t}_{0}}
$$

where $f(x)=$ ax. Acceleration will be Eq. (19),

$$
a=\lim _{t_{0} \rightarrow 0} \frac{a t-a t_{0}}{t-t_{0}}
$$

where $f(x)=a x$. Because of these reasons, if it is required to integrate Eq. (16a), it will be Eq. (18).

$$
\mathrm{x}(\mathrm{t})=\int\left(\mathrm{v}_{0}+\mathrm{at}\right) \mathrm{d}_{\mathrm{t}}=\int\left(\mathrm{v}_{0}+\frac{\mathrm{d}}{\mathrm{d}_{\mathrm{t}}} \mathrm{x}\right) \mathrm{d}_{\mathrm{t}}=\mathrm{x}_{0}+\mathrm{x}=\Delta \mathrm{x}
$$

Eq. (17), Eq. (18) and Eq. (19) are the most basic pillars for like these calculations. As constant acceleration as absolute is not possible, a $\int \mathrm{t} \mathrm{d} t=a t^{2} / 2$ is not possible, and there is one more option as well as Eq. (21).

$$
\mathrm{x}(\mathrm{t})=\int\left(\mathrm{v}_{0}+\mathrm{at}\right) \mathrm{d}_{\mathrm{t}}=\int\left(\mathrm{v}_{0}+\mathrm{v} \frac{\mathrm{d}}{\mathrm{d}_{\mathrm{t}}} \mathrm{t}\right) \mathrm{d}_{\mathrm{t}}=\mathrm{x}_{0}+\mathrm{x}=\Delta \mathrm{x}
$$

\subsubsection{Timeless velocity equations}

Handle the motions which have no observational outer space initial velocity. If the time over Eq. (13) is placed on Eq. (14) for their magnitudes, the timeless velocity equation becomes Eq. (22).

$$
\mathrm{v}^{2}=\mathrm{ax}
$$

Also it can be presented as Eq. (22a).

$$
\mathrm{v}^{2}=(\mathbf{a} \cdot \mathbf{x})
$$

Thereupon $\mathrm{v}^{2}=2$ ax equation is wrong as well.

Handle the motions with initial velocity. Torricelli equation $v_{\mathrm{f}}^{2}=\mathrm{v}_{\mathrm{i}}^{2}+2 \mathrm{ax}$ gives wrong results because of $\mathrm{v}^{2}=2 \mathrm{ax}$ equation. If $\mathrm{v}_{\mathrm{i}}$ is the initial velocity, $\mathrm{v}_{\mathrm{g}}$ is the gained velocity different than the first velocity, $v_{f}$ is the final velocity; then the equations must be as $\mathrm{v}_{\mathrm{f}}=\mathrm{v}_{\mathrm{i}}+\mathrm{v}_{\mathrm{g}}$ and $\mathrm{v}_{\mathrm{g}}=\mathrm{a}_{\mathrm{g}} \mathrm{t}$ equations. If they are the assembly elements, then for $v_{f}^{2}=\left(v_{i}+a_{g} t\right)^{2}$, it becomes Eq. (23).

$$
\mathrm{v}_{\mathrm{f}}^{2}=\mathrm{v}_{\mathrm{i}}^{2}+2 \mathrm{v}_{\mathrm{i}} \mathrm{ag}_{\mathrm{g}} \mathrm{t}+\mathrm{a}_{\mathrm{g}}^{2} \mathrm{t}^{2}
$$

If the total distance taken is $\mathrm{x}_{\mathrm{f}}, \mathrm{x}_{\mathrm{i}}$ is the distance taken during the same $t$ time by the initial velocity, $x_{g}$ is the distance taken by the gained velocity which is different than the first velocity during the same t time, then the equations must be $\mathrm{x}_{\mathrm{f}}=\mathrm{x}_{\mathrm{i}}+\mathrm{x}_{\mathrm{g}}, \mathrm{x}_{\mathrm{g}}=\mathrm{ag}_{\mathrm{g}} \mathrm{t}^{2}$ and thus $\mathrm{t}^{2}=\left(\mathrm{x}_{\mathrm{f}}-\mathrm{x}_{\mathrm{i}}\right) / \mathrm{ag}_{\mathrm{g}}$ equations. If this time is placed on the place on Eq. (23), then the equation becomes $\mathrm{v}_{\mathrm{f}}^{2}=\mathrm{v}_{\mathrm{i}}^{2}+2 \mathrm{v}_{\mathrm{i}} \mathrm{ag}_{\mathrm{g}} \mathrm{t}+\mathrm{a}_{\mathrm{g}} \mathrm{x}_{\mathrm{f}}-\mathrm{ag}_{\mathrm{g}} \mathrm{x}_{\mathrm{i}}$. For $\mathrm{v}_{\mathrm{i}} \mathrm{t}=\mathrm{x}_{\mathrm{i}}$ equation, it will be as $2 \mathrm{v}_{\mathrm{i}} \mathrm{ag}_{\mathrm{g}} \mathrm{t}=2 \mathrm{x}_{\mathrm{i}} \mathrm{a}_{\mathrm{g}}$, and the timeless velocity equation which is with an initial velocity will be as Eq. (23a),

$$
\mathrm{v}_{\mathrm{f}}^{2}=\mathrm{v}_{\mathrm{i}}^{2}+\mathrm{ag}_{\mathrm{g}}\left(\mathrm{x}_{\mathrm{i}}+\mathrm{x}_{\mathrm{f}}\right)
$$

or will be as Eq. (23b) the below.

$$
\mathrm{v}_{\mathrm{f}}^{2}=\mathrm{v}_{\mathrm{i}}^{2}+\mathrm{ag}_{\mathrm{g}}\left(2 \mathrm{x}_{\mathrm{i}}+\mathrm{x}_{\mathrm{g}}\right)
$$

In the same manner, over $v_{f}^{2}$, it will be as Eq. (24),

$$
\mathrm{v}_{\mathrm{f}}^{2}=\mathrm{v}_{0}^{2}+\mathrm{v}_{\mathrm{g}}^{2}+2\left(\mathbf{x}_{\mathbf{0}} \cdot \mathbf{v}_{\mathrm{g}}\right)
$$

where $\mathbf{v}_{\mathbf{g}}=\mathbf{a}_{\mathbf{g}} \mathrm{t}, \mathbf{x}_{\mathbf{0}}=\mathbf{v}_{\mathbf{0}} \mathrm{t}$ and the other equations are as follows.

$$
\begin{gathered}
\mathbf{v}_{\mathbf{f}} \cdot \mathbf{v}_{\mathbf{f}}=\left(\mathbf{v}_{\mathbf{0}}+\mathbf{a}_{\mathbf{g}} \mathrm{t}\right) \cdot\left(\mathbf{v}_{\mathbf{0}}+\mathbf{a}_{\mathbf{g}} \mathrm{t}\right) \\
\mathrm{v}_{\mathrm{f}}^{2}-\mathrm{v}_{0}^{2}=2 \mathrm{t}\left(\mathbf{a}_{\mathrm{g}} \cdot \mathbf{v}_{\mathbf{0}}\right)+\mathrm{a}_{\mathrm{g}}^{2} \mathrm{t}^{2}
\end{gathered}
$$

\subsubsection{Deceleration}

As constant acceleration motion is not possible, then for two different $\mathrm{t}$ values in $\mathrm{x}(\mathrm{t})=\mathrm{at}^{2}$ and $\mathrm{v}(\mathrm{t})=$ at functions, will change functions even for the smallest time change [1]. For $\mathrm{f}(\mathrm{x})=\mathrm{ax}^{2}$ where $\mathrm{a}$ is any acceleration which is fixed or not, over $\mathrm{f}\left(\mathrm{x}_{\mathrm{n}+1}\right)=\mathrm{f}\left(\mathrm{x}_{\mathrm{n}}\right)$ equation where $\mathrm{x}_{\mathrm{n}+1}>\mathrm{x}_{\mathrm{n}}$ and for the same distance taken magnitude between two different times, the equation becomes Eq. (25).

$$
\frac{a_{n}}{a_{n+1}}=\frac{t_{n+1}^{2}}{t_{n}^{2}}
$$

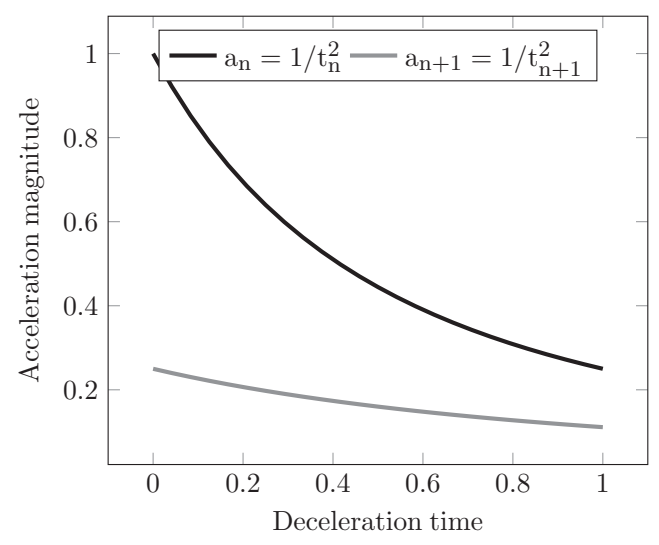

Fig. 3: Deceleration for a limited interval

There is a presentation of the motion as Fig. 3. Hence it can be said that, $a_{n}$ which is one of the previous acceleration magnitudes is always bigger than the next $a_{n+1}$ magnitude; therefore the universe is decelerating from an infinite value as a virtual part of absolute space, in a time interval and by having emergence time priority for each small point of emergence area. It has to emerge by frequency, and there cannot emerge a point which has absolute emptiness namely nothingness. Already acceleration is not possible from zero point namely from nothingness because of previous motion, and already if constant speed and acceleration motion are not possible, it means that already motion emerges by parts over time at infinite frequency which means no constant speed even for infinite small work part. Otherwise, it requires to be absolute. 
An uncertain matter can only emerge in a time interval by a deceleration from infinity, as a part of infinity, by repeating the same magnitude assumed infinite small motion. As a result of the deceleration, there cannot emerge an absolute emptiness. Matter emerges over this emergence space. Because of emerging uncertainty by an infinite frequency, matter basically does not have work potential. An absolute must work constantly even in the smallest time, instead of uncertain worker.

\section{Inference}

\subsection{Imaginary time requirement}

\subsubsection{Excessive time}

Handle an equation like Eq. (26).

$$
\infty+\mathrm{t}-\mathrm{t}=\infty
$$

It means that an $E$ energy is created over absolute space in $t$ time as virtual part of space, and it got lost after that in the same magnitude t time. It seems that there is no problem; but actually when the event's actual emerging function is written as Eq. (27)

$$
\infty+\sum_{n=1}^{n}\left(t_{n}-t_{n-1}\right)-t=\infty
$$

where $t_{n}=t$, there will emerge two different options according to the calculation method. The sum's result is either $t-t_{0}$ where $t_{0}=0$ or $t-t_{0}$ where $t_{0}$ is a threshold value. For $\mathrm{t}_{0}=0$, it can be said, that it is impossible; because there are infinite time intervals even for each magnitude time interval; so if the result of $t_{1}-t_{0}$ is not 0 , then it means even $t_{1}$ has sub times, and the same sum must be repeated for $t_{1}$ and even for its infinite parts; so the equation turns into Eq. (28).

$$
\mathrm{t}-\mathrm{t}_{0}=\mathrm{t}
$$

Hence time is only defined if it is

$$
\lim _{t_{0} \rightarrow 0}\left(t-t_{0}\right)=t
$$

Over Eq. (28), if both of the sides are squared, it becomes $\mathrm{t}_{1}=\frac{\mathrm{t}_{0}}{2}$. If it is put on Eq. (28), the other root becomes $\mathrm{t}_{2}=-\frac{\mathrm{t}_{0}}{2}$ over $\frac{\mathrm{t}_{0}}{2}-\mathrm{t}_{0}=\mathrm{t}$. When the both roots are multiplied by each other to create the main function which has these two roots, it becomes $-\mathrm{t}_{0}^{2} / 4=\mathrm{t}$ and finally becomes Eq. (30).

$$
\mathrm{t}_{0}=2 \mathrm{i} \sqrt{\mathrm{t}}
$$

It can be said that time is always negative for any positive or negative $t_{0}$. If it is put on Eq. (28), it becomes Eq. (31),

$$
-\mathrm{t}_{0}^{2}-4 \mathrm{t}_{0}=4 \mathrm{t}
$$

where

$$
\begin{gathered}
\mathrm{t}_{0}=-2 \mathrm{n} \\
\mathrm{t}=2 \mathrm{n}-\mathrm{n}^{2}
\end{gathered}
$$

being $\mathrm{n} \in \mathbb{Z}$, for $\mathrm{t} / \mathrm{t}_{0}=-1$ instead of $0 / 0$ uncertainty, where $\mathrm{n}=0$.

If the same is applied for velocity, the distance taken of $\mathrm{x}_{0}$ becomes

$$
\mathrm{x}_{0}=-4 \sqrt{\mathrm{vt}}
$$

where $\mathrm{v}_{0}=2 \mathrm{i} \sqrt{\mathrm{v}}$. It is defined; so it means that whatever already there was motion as uncertain motion. This requires an imaginary time; because existence can only be either absolute or uncertain.

Hence, to be defined of $t$, it is required that $t_{0}$ must be 0 ; but when it is done, also $t$ becomes 0 . For $t=0$, also velocity and distance taken become zero; therefore, $t$ must has another root that if Eq. (31) is checked, when it becomes $n=0$, then $t_{0}$ and $t$ become 0 ; but also for $n=2$, $t$ becomes zero; but there is an imaginary time that there is a motion in a time interval. This untimely manner creates a second time beyond to be suddenly of it since it is a root of time; so it can be said that, even there was no motion in outer space, there was a repeated motion in imaginary time which is not absolute; but also is not the same with time. Right this point, deterministic image in the imaginary time appears, is lengthened by a repeat frequency according to using energy by creating real time. Each previous work is done timeless according to $t-t_{0}=t$; because here, it is $t_{n}-t_{n-1}=t_{0}$; so each consecutive time is equal to each other and 0 ; so also is $t_{0}=t$.

Imaginary time is a potential; you know everything is there but there is no motion. Below stated calculations for imaginary time analysis by graphs to understand there must be information before it created. Namely numbers are not important. The basic logic is important and they are just representation.

\section{Warning}

\subsubsection{Derivative at infinite}

Motion emerges by parts over time at infinite frequency which does not bring matter out infinite energy but renders uncertain as a virtual part. It could be in this way. Otherwise, it requires to be absolute; then the functions of time as equations of motion emerge as Eq. (33), Eq. (33a) and Eq. (33b). They give a single result for a single infinite derivation which is done untimely manner as one-piece by visiting the different functions they work over them. Here, actual functions of time can be any function if it is compatible with the rule of displacement; because the smallest same part will be used for each infinite small frequency again and again.

$$
\begin{aligned}
& \mathrm{x}=\mathrm{vt}=0=\lim _{\mathrm{n} \rightarrow \infty} \frac{\mathrm{d}^{\mathrm{n}}}{\mathrm{d}_{\mathrm{t}}^{\mathrm{n}}}(\mathrm{x}(\mathrm{t})) \\
& \mathrm{v}=\mathrm{at}=0=\lim _{\mathrm{n} \rightarrow \infty} \frac{\mathrm{d}^{\mathrm{n}}}{\mathrm{d}_{\mathrm{t}}^{\mathrm{n}}(\mathrm{v}(\mathrm{t}))} \\
& \mathrm{x}=\mathrm{at}^{2}=0=\lim _{\mathrm{n} \rightarrow \infty} \frac{\mathrm{d}^{\mathrm{n}}}{\mathrm{d}_{\mathrm{t}}^{\mathrm{n}}}(\mathrm{x}(\mathrm{t}))
\end{aligned}
$$

where $\mathrm{x}(\mathrm{t})=\mathrm{vt}, \mathrm{x}(\mathrm{t})=\mathrm{at} \mathrm{t}^{2}, \mathrm{v}(\mathrm{t})=\mathrm{at}$, and a and $\mathrm{v}$ are constants as a number; therefore it means time has some roots. Otherwise, it would not become like vt $=0$, $\mathrm{at}^{2}=0$ or at $=$ 0 . Even if actual functions of velocity, distance taken and acceleration were different, frequency function would be the same as $f(t)=1 / t$. For this equation, it becomes Eq. (33c),

$$
1 / t=\lim _{n \rightarrow \infty} \frac{d^{n}}{d_{t}^{n}}(f(t))
$$

If it is edited, it becomes Eq. (33d) over Eq. (33e) where $n \in \mathbb{Z}$ and $n>0$.

$$
\begin{gathered}
\mathrm{t}=\lim _{\mathrm{n} \rightarrow \infty}(-1)^{\mathrm{n}+1} \sqrt[\mathrm{n}+1]{(\mathrm{n}+1) !} \\
\frac{1}{\mathrm{t}}=\frac{(\mathrm{n}+1) !(-1)^{\mathrm{n}+1}}{\mathrm{t}^{\mathrm{n}+2}}
\end{gathered}
$$


Here, $(-1)^{\mathrm{n}+1}$ cannot be taken to the outside like -1 or +1 ; because $n$ always changes. The difference is that it does not turn it into complex number.

Matter emerges from imaginary time by increasing frequency from its information state. Actually, each small wave part decreases from an infinite value; but as there are numerous wave parts which emerge and increase over time until 1 second, as a result, its frequency or repeat is going to be increased.

\section{Warning}

Here, $\lim _{n \rightarrow \infty}\left(\log _{e} n-\log _{e} t\right)=1$ when $(-1)^{n+1}$ is ignored It is interesting, that actually $t$ gives positive and negative values according to being positive or negative of $n$; but the function converges to the Euler number as irrational value by

$$
\mathrm{e}=\lim _{\mathrm{n} \rightarrow \infty} \frac{\mathrm{n}}{\sqrt[n+1]{(\mathrm{n}+1) !}}
$$

$(-1)^{\mathrm{n}+1}$ on Eq. (33d) means that acceleration direction would change in time if it was done in a time interval; however there is no time interval to emerge of this because of infinite derivatives which are done untimely manner; so it gives its absolute characteristic. Even if there is no derivative done in a time interval, it acts such that for partial information namely for finite matter, our universe; so by using ratio test for both $\mathrm{t}_{+}=\lim _{\mathrm{n} \rightarrow \infty}+\sqrt[2 \mathrm{n}]{(2 \mathrm{n}) !}$ and $\mathrm{t}_{-}=\lim _{\mathrm{n} \rightarrow \infty}-\sqrt[2 \mathrm{n}-1]{(2 \mathrm{n}-1) !}, \mathrm{t}_{-}$diverges in absolute value from the result of $t_{+}$for the same derivation number; therefore when an addition is done for the assumed smallest two consecutive parts, time seems as always negative, and the equation of velocity turns into Eq. (33f).

$$
\mathbf{v}=-\mathbf{a t}
$$

The actual reason of that negativity of the formulas like $\mathrm{F}=-\mathrm{ma}$ is secret element of the formula. $\mathrm{F}=$ ma or $\mathrm{F}=-\mathrm{ma}$ is general formula of the motion as presentation that actually it is like a closed presentation of a chain. Because of uncertainty, actually there is no absolute threshold value. Existent smallest force can move existence biggest mass instantly even if the emerging acceleration value will too small; so if there is a motion, there are minimum 3 elements emerge in such a formula like $\mathrm{F}=-\mathrm{m}_{1} \mathrm{a}_{1}+\mathrm{m}_{2} \mathrm{a}_{2}$ or $-\mathrm{F}=\mathrm{m}_{1} \mathrm{a}_{1}-\mathrm{m}_{2} \mathrm{a}_{2}$ because of that reason; because nothing can apply force without fulcrum. If you blow air, then air will move; but also you will move the earth instantly. 1 of the 3 elements is ignored since will get a negligible value; but actually it is always there and renders formulas in the simplest forms like $\mathrm{F}=-$ ma.

\section{Warning}

\subsubsection{Presentation by the relation between the functions of time}

As analysis together to learn the nature of the formation by a different way, as $\mathrm{v}(\mathrm{t})=$ at and $\mathrm{x}(\mathrm{t})=\mathrm{at}^{2}$ functions change by acceleration; to know velocity, distance taken and acceleration change by which ratio, we must follow the area between the two functions; because a is an intersection point on $y$ axis for both of the functions.

The area for changing time and acceleration values is
Eq. (34).

$$
\mathrm{A}=\int\left(\mathrm{ax}-\mathrm{ax} \mathrm{x}^{2}\right) \mathrm{d}_{\mathrm{x}}=\frac{\Delta \mathrm{vt^{2 }}}{2}-\frac{\Delta \mathrm{vt}^{3}}{3}
$$

The ratio between the area and distance taken becomes Eq. (35).

$$
\mathrm{A} / \Delta \mathrm{x}=\frac{\mathrm{t}}{2}-\frac{\mathrm{t}^{2}}{3}
$$

For these values, the graph becomes Fig. 4. To determine the

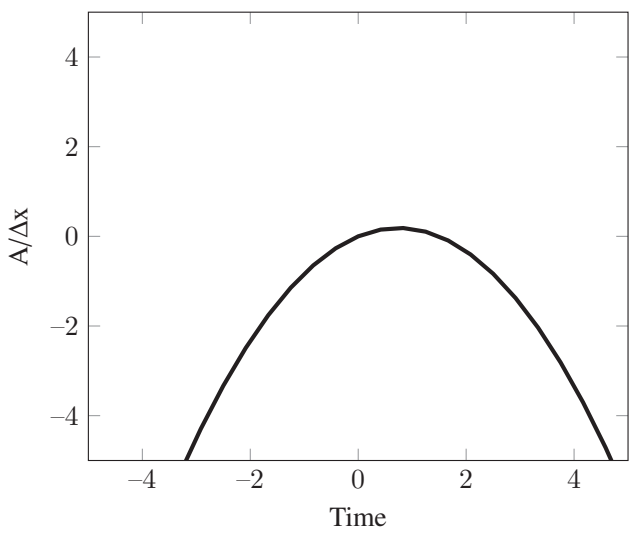

Fig. 4: $\mathrm{A} / \Delta \mathrm{x}$ function

intersection point of $\mathrm{A}$ and $\Delta \mathrm{x}$, for $\mathrm{A}-\Delta \mathrm{x}=0$ equation, it will be as Eq. (35a).

$$
\mathrm{A}-\Delta \mathrm{x}=2 \mathrm{t}^{2}-3 \mathrm{t}+6
$$

Over the standard quadratic equation of $\mathrm{ax}^{2}+\mathrm{bx}+\mathrm{c}$, for the roots over $\mathrm{x}_{1,2}=\frac{-\mathrm{b} \pm \sqrt{\Delta}}{2 \mathrm{a}}$ equation where $\Delta=\mathrm{b}^{2}-4 \mathrm{ac}$, the discriminant is as $\Delta=-39$ for Eq. (35a). For $\Delta<0$, there are no real roots and the quadratic equation has two complex roots as Eq. (35b).

$$
\mathrm{x}_{1,2} \approx+0.7500 \pm 1.5612 \mathrm{i}
$$

According to Eq. (35a), there is no positive real number intersection point; so $\mathrm{A}$ and $\Delta \mathrm{x}$ never intersect.

The ratio between the area and velocity is Eq. (36).

$$
\mathrm{A} / \Delta \mathrm{v}=\frac{\mathrm{t}^{2}}{2}-\frac{\mathrm{t}^{3}}{3}
$$

For these values, the graph is Fig. 5.

To determine the intersection point of $\mathrm{A}$ and $\Delta \mathrm{v}$, for $\mathrm{A}-$ $\Delta \mathrm{v}=0$ equation it will be as Eq. (36a).

$$
\mathrm{A}-\Delta \mathrm{v}=2 \mathrm{t}^{3}-3 \mathrm{t}^{2}+6
$$

By using Cardano's formula for the standard equation of $\mathrm{ax}^{3}+\mathrm{bx}^{2}+\mathrm{cx}+\mathrm{d}=0$, when the cubic equation as $2 \mathrm{t}^{3}-3 \mathrm{t}^{2}+6=0$ is divided by $\mathrm{a}=2$ as Eq. (36b),

$$
\mathrm{t}^{3}-\frac{3 \mathrm{t}^{2}}{2}+3=0
$$

and it is substituted $t=y-\frac{b}{3 a}$, where $p=-\frac{b^{2}}{3 a^{2}}+\frac{c}{a}$ and $q=\frac{2 b^{3}}{27 a^{3}}-\frac{b c}{3 a^{2}}+\frac{d}{a}$, the discriminant of the cubic equation 


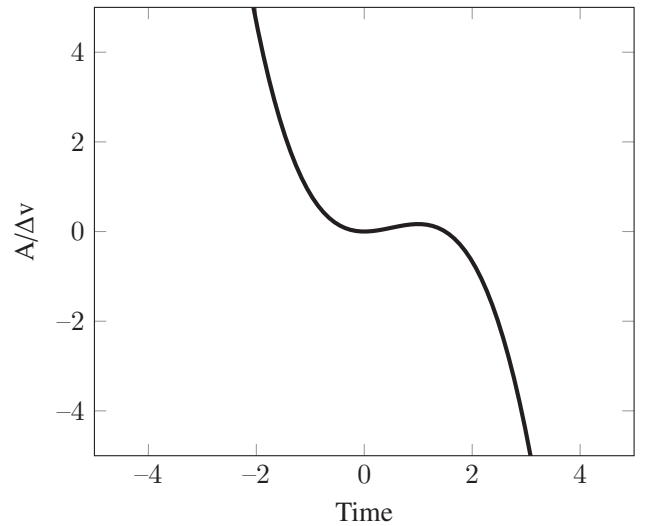

Fig. 5: $\mathrm{A} / \Delta \mathrm{v}$ function

which is $\Delta=\left(\frac{\mathrm{p}}{3}\right)^{3}+\left(\frac{\mathrm{q}}{2}\right)^{2}$ becomes $\Delta=15 / 8$. As it is $\Delta>0$, one root is real and two are complex conjugates. The real root is as Eq. (36c) for $\mathrm{t}_{\mathrm{r}}=\alpha+\beta$,

$$
\mathrm{t}_{\mathrm{r}} \approx-1.0786
$$

where $\alpha=\sqrt[3]{-\frac{\mathrm{q}}{2}+\sqrt{\Delta}}$ and $\beta=\sqrt[3]{-\frac{\mathrm{q}}{2}-\sqrt{\Delta}}$. The other two roots of the cubic equation are Eq. (36d) for $\mathrm{t}_{2,3}=-\frac{\alpha-\beta}{2} \pm$ $\frac{\mathrm{i} \sqrt{3}(\alpha-\beta)}{2}$.

$$
\mathrm{t}_{2,3} \approx+1.2893 \pm 1.0578 \mathrm{i}
$$

According to Eq. (36a), there is no positive real number intersection point; so $\mathrm{A}$ and $\Delta \mathrm{v}$ never intersect at the positive side.

The ratio between the area and acceleration is Eq. (37).

$$
\mathrm{A} / \Delta \mathrm{a}=\frac{\mathrm{t}^{3}}{2}-\frac{\mathrm{t}^{4}}{3}
$$

For these values, the graph is Fig. 6. To determine the inter-

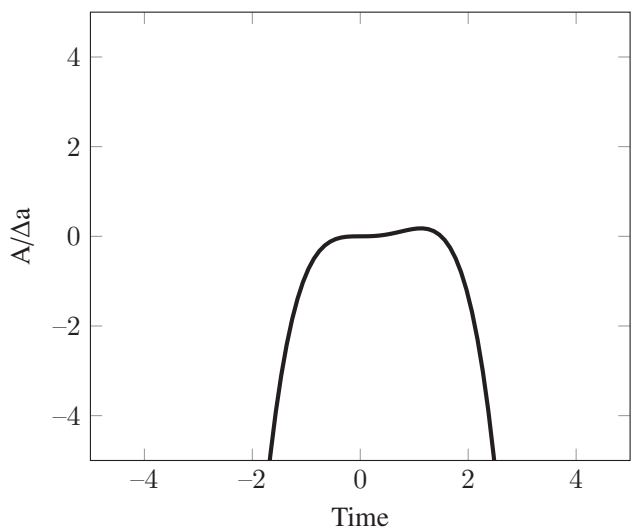

Fig. 6: $\mathrm{A} / \Delta \mathrm{a}$ function

section point of $\mathrm{A}$ and $\Delta \mathrm{a}$, for $\mathrm{A}-\Delta \mathrm{a}=0$ equation it will be as Eq. (37a).

$$
\mathrm{A}-\Delta \mathrm{a}=2 \mathrm{t}^{4}+3 \mathrm{t}^{3}+6
$$

Over the standard quartic equation of $a x^{4}+b^{3}+c x^{2}+$ $\mathrm{dx}+\mathrm{e}=0$ by using the substitution $\mathrm{t}=\frac{\mathrm{b}}{4 \mathrm{a}}$, we get the depressed equation $\mathrm{t}^{4}+\mathrm{pt}^{2}+\mathrm{qt}+\mathrm{r}=0$ where $\mathrm{p}=\frac{8 \mathrm{ac}-3 \mathrm{~b}^{2}}{8 \mathrm{a}^{2}}$, $q=\frac{8 a^{2} d+b^{3}-4 a b c}{8 a^{3}}, r=\frac{16 a b^{2} c-64 a^{2} b d-3 b^{4}+256 a^{3} e}{256 a^{4}}$. As it is $\mathrm{q}=0$ for Eq. (37a), $\mathrm{t}^{4}+\mathrm{pt}^{2}+\mathrm{qt}+\mathrm{r}=0$ becomes $\mathrm{a}$ bi-quadratic equation as $\mathrm{t}^{4}+\mathrm{pt}^{2}+\mathrm{r}=0$. The roots of the quartic equation are Eq. (37b) for $\mathrm{t}_{1,2}= \pm \sqrt{\frac{-\mathrm{p}-\sqrt{\mathrm{p}^{2}-4 \mathrm{r}}}{2}}$, and are Eq. (37c) for $\mathrm{t}_{3,4}= \pm \sqrt{\frac{-\mathrm{p}+\sqrt{\mathrm{p}^{2}-4 \mathrm{r}}}{2}}$.

$$
\begin{aligned}
& \mathrm{t}_{1,2} \approx-0.65934 \pm 0.866004 \mathrm{i} \\
& \mathrm{t}_{3,4} \approx+1.40935 \pm 0.738938 \mathrm{i}
\end{aligned}
$$

According to Eq. (37a), there is no positive real number intersection point; so $\mathrm{A}$ and $\Delta \mathrm{a}$ never intersect.

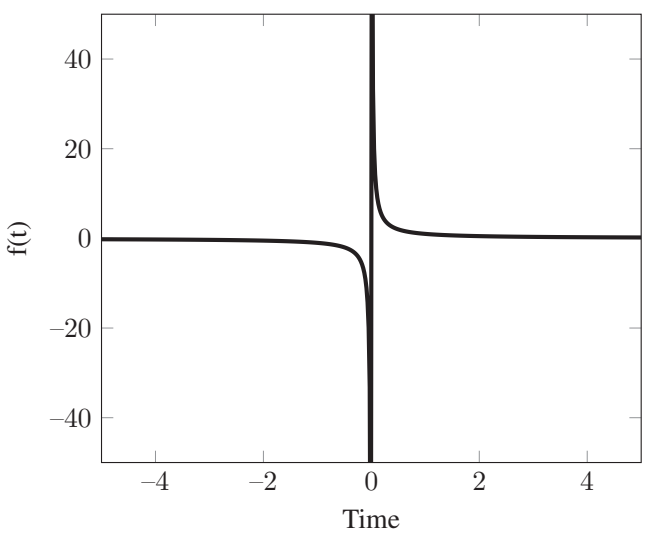

Fig. 7: $f(t)$ function for $x: 1$ and $y: 10$ scale

As constant speed movement is not possible, motion emerges by infinite small parts in a time interval. It causes frequency. For $(\mathrm{A} / \Delta \mathrm{v}) /(\mathrm{A} / \Delta \mathrm{x})$ equation, it will become Eq. (38) as frequency since frequency is $\mathrm{v} / \mathrm{x}=1 / \mathrm{t}=\mathrm{f}$.

$$
f(t)=\frac{3-2 t}{3 t-2 t^{2}}
$$

The graph of $f(t)$ becomes Fig. 7.

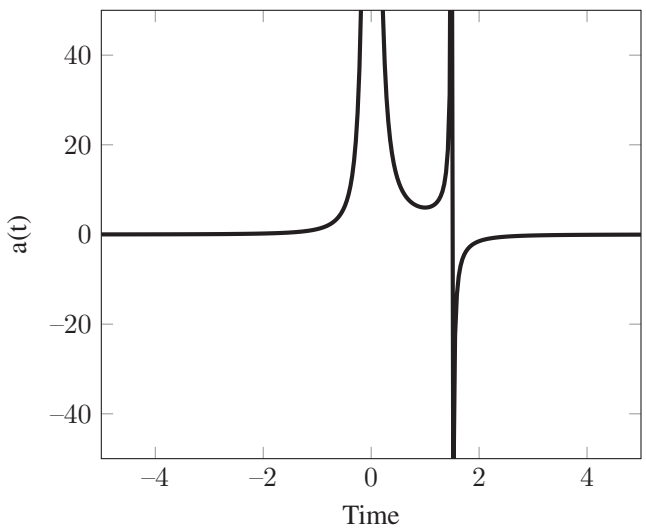

Fig. 8: a(t) function for $\mathrm{x}: 1$ and $\mathrm{y}: 10$ scale

According to $\Delta \mathrm{a} / \Delta \mathrm{t}=(1 / \mathrm{f}) /(\mathrm{A} / \Delta \mathrm{a})$ equation, $\Delta \mathrm{a} / \Delta \mathrm{t}$ becomes Eq. (39), and know that it is self-inverse function and 
$\mathrm{a}^{-1}(\mathrm{t})$ renders possible a closed curve.

$$
a(t)=\frac{6}{3 t^{2}-2 t^{3}}
$$

The graph of $\mathrm{a}(\mathrm{t})$ becomes Fig. 8 .

The other functions of time turn into the following equations according to changing rule in time of acceleration which is Eq. (39). Velocity function turns into Eq. (40).

$$
\mathrm{v}(\mathrm{t})=\frac{6}{3 \mathrm{t}-2 \mathrm{t}^{2}}
$$

The graph of $v(t)$ becomes Fig. 9.

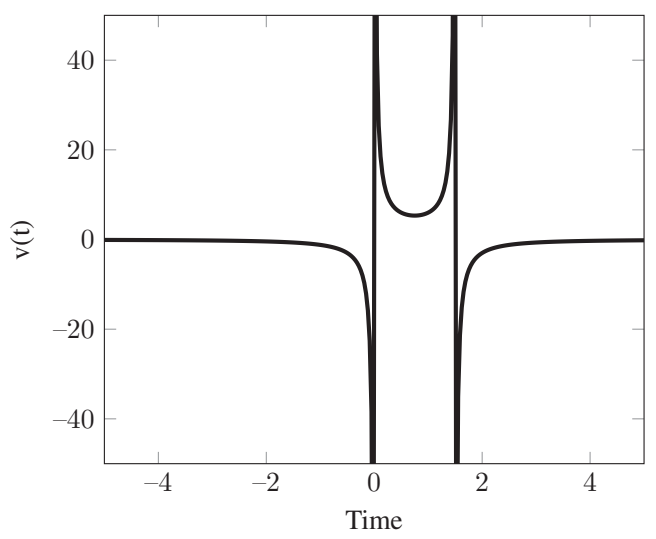

Fig. 9: v(t) function for $\mathrm{x}: 1$ and $\mathrm{y}: 10$ scale

Distance taken function turns into Eq. (41), and know that it is self-inverse function and $\mathrm{x}^{-1}(\mathrm{t})$ renders possible a closed curve.

$$
\mathrm{x}(\mathrm{t})=\frac{6}{3-2 \mathrm{t}}
$$

The graph of $\mathrm{x}(\mathrm{t})$ becomes Fig. 10 .

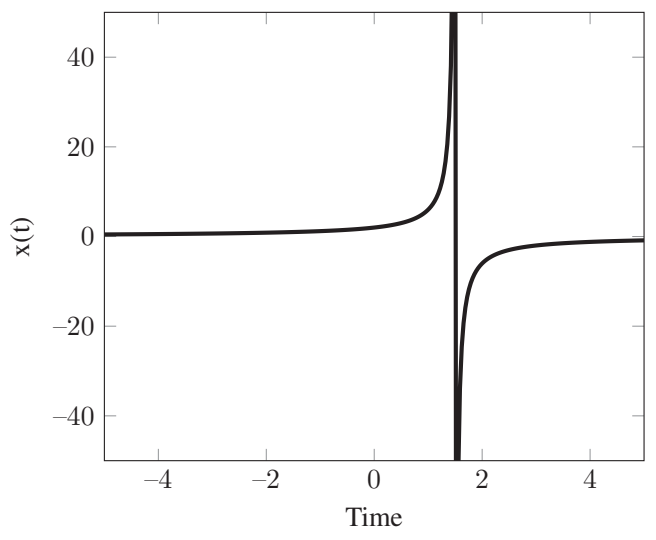

Fig. 10: $\mathrm{x}(\mathrm{t})$ function for $\mathrm{x}: 1$ and $\mathrm{y}: 10$ scale

According to $\mathrm{x}(\mathrm{t})$ graph, it seems that at the moment of $\mathrm{t}=0$ second, already there had been motion. Motion had not been emerged from nothingness. To analysis the condition, we must analysis the roots of the functions. Eq. (40) and Eq. (41) have the same roots with Eq. (35) and Eq. (36); so they have no real root. Also Eq. (39) has the same roots with Eq. (37); so only a(t) has real root, and it has a single root as $t_{i} \approx-1.0786$. It seems that according to a(t) function, there is an imaginary time $t_{i}$ as the single root for any function of motion as irrational number $t \approx-1.0786$. Also it means that as the time is uncertain, it was not an absolute energy and motion; therefore it was also created but was without time. At this time, acceleration is 1 ; so velocity becomes $\mathrm{v}(\mathrm{t}) \approx-1.0786$. For positive acceleration as vector quantity, if velocity is negative, then already there is an imaginary time mentioned as there shall no place. There is no time difference between two motions even as an threshold value. Absolute 0 point seems impossible and means that matter always together with heat. For this imaginary time, also distance taken is positive as vector quantity. It means that distance taken and velocity were in reverse directions. Frequency for these values will be $v(\mathrm{t}) \approx-0.92712$ for $v=1 / \mathrm{t}$ that it seems also time is vector quantity.

As the infinite never works for any size work, as any act is together with probability, also it does not matter for the infinite, that probability is high or low. The infinite can create the existent highest or lowest probability at the same difficulty; so do not say, that its probability is very low for something while you are reading the mind of God.

\section{Warning}

As it can be seen over Fig. 10, distance taken does not reach infinite for $\left[\mathrm{t}_{\mathrm{i}}, 0\right]$; whereas $\mathrm{a}(\mathrm{t}), \mathrm{v}(\mathrm{t})$ and $\mathrm{f}(\mathrm{t})$ which are the other functions of time increase into infinite value for the same time interval. This seems as paradox; but it is because of the creation of infinite. As everything is element of infinite, since we cannot add anything in or detract from infinite, the infinite does not work for any size work; so work done is always zero for the infinite. The smallest work and the biggest work are at the same difficulty; so at $t=0$ second point, the infinite creates after the thinking which causes a potential difference without space wave. Creating from nothingness requires this. You need infinite energy even for the smallest mass part; so the infinite uses all the energy it has. As frequency does not become 0 for any work after $t=0$ second, it means that the motion does not be forgotten. Creation always only happens at $t=0$ second by infinite frequency, by infinite precision and by the simple functions like $\Delta \mathrm{x}=\Delta \mathrm{v} \Delta \mathrm{t}$ and $\Delta \mathrm{v}=\Delta \mathrm{a} \Delta \mathrm{t}$; because this is rendering visible process; but even in imaginary time, matter was not absolute. This seems as paradox but it is not. There is a third option as imaginary time.

\subsection{Kinetic energy and momentum equations}

\subsubsection{Theoretic analysis of area}

\section{a) Dimensional emergence}

To analysis dimensional density, for Eq. (42),

$$
\mathrm{d}(\mathrm{n})=\sum_{\mathrm{t}=1}^{\infty} \frac{\mathrm{A}(\mathrm{n})}{\mathrm{A}(\mathrm{n}+1)}
$$

the equation turns into (42a),

$$
\mathrm{d}(\mathrm{n})=\frac{\zeta(1)}{\mathrm{v}}
$$

where $\mathrm{A}(\mathrm{n})=\mathrm{r}^{\mathrm{n}}(\mathrm{t}), \mathrm{n}>0, \mathrm{~d}(\mathrm{n})$ is density of existence area of a dimension in the next dimension, and $\mathrm{r}(\mathrm{t})=\mathrm{vt}$ as time is restrictive to exist at anywhere at the same time along infinite space, for a fixed and limited velocity. $d(n)$ by this rule is always divergent; so over Eq. (42b),

$$
\lim _{n \rightarrow \infty} \sum_{t=1}^{\infty} \frac{A(n)}{A(n+1)}=\infty
$$


it can be said that any previous dimension which is assumed as it creates out next one exists at infinite density in the next dimension by a limited velocity; but as the relation between different dimensions, the equation turns into (43),

$$
\mathrm{d}(\mathrm{n})=\sum_{\mathrm{t}=1}^{\infty} \frac{\mathrm{A}(\mathrm{n})}{\mathrm{A}(\mathrm{n}+\mathrm{m})}
$$

where $m>1$, and it turns into (43a),

$$
\mathrm{d}(\mathrm{n})=\frac{\zeta(\mathrm{m})}{\mathrm{v}^{\mathrm{m}}}
$$

and is convergent. It means that a sub-dimension exists. As a result, for (43b) over (43a) where $\mathrm{n}=1$ as 1 dimension,

$$
\lim _{\mathrm{m} \rightarrow \infty} \frac{\zeta(\mathrm{m})}{\mathrm{v}^{\mathrm{m}}}=0
$$

any dimension or actually 1 dimension has no density in absolute space, and so cannot exist in infinite dimensional space. Also lower dimensions' density decreases for higher dimensions that if a next dimension was created out by the previous one or previous ones, this condition would be in the exact opposite way. This means that emergence area and its elements emerge on their own space, that it requires to be determined before. If it cannot create a next dimension out, and also if it cannot be a part of infinite dimensional space as a formation part of infinite space, it means that, area is absolute. Subdimension cannot form the next one. It was always there, and there is an appearance, and drop from infinity. There is no 0 dimension, and the other dimension appears as relatively in different dimension by having different density.

By another method, if area is conservative, then for two dimensional medium, it becomes Eq. (44),

$$
\mathrm{dxdy}=0
$$

where $\mathrm{ds}=\mathrm{dx}+\mathrm{dy}, \mathrm{ds}^{2}=\mathrm{dx}^{2}+\mathrm{dy}^{2}$ and thus $\mathrm{dx}^{2}+\mathrm{dy}^{2}=$ $(d x+d y)^{2}$. Here, even if the two components always change, sum of them is always conserved for any physical value for example as $\mathrm{p}=\mathrm{p}_{\mathrm{x}}+\mathrm{p}_{\mathrm{y}}$ and $\mathrm{p}^{2}=\mathrm{p}_{\mathrm{x}}^{2}+\mathrm{p}_{\mathrm{y}}^{2}$. It means two dimensional space is not possible.

For the above stated conditions, it seems that dimension is movement way which does not mean area as it is not abstract, and is vector quantity. Each dimension has a unique way relatively to any other dimension. As area, it is sum of dimensional positive and negative movement lengths, and also is vector quantity. Displacement vector is only able to emerge in different ways as much as dimension numbers which area has them; therefore area is defined if and only if is $\mathbf{A}: \mathbb{R}^{\mathrm{n}} \rightarrow \mathbb{R}^{\mathrm{n}}$ as vector quantity or if is $\mathbf{A}: \mathbb{R}^{\mathrm{n}} \rightarrow \mathbb{R}$ as scalar magnitude, where $\mathrm{n}$ is dimension number which the vector field $\mathbf{A}$ has; thus $\mathbf{A}$ emerges as $\mathrm{n}$ dimensional area which means allows objects to move in different ways on displacement vector line; otherwise objects can only move to the aimed point by wandering and by a big latency.

As motion emerges in a time interval, it must always has limited speed. As each dimension has unique way, emergence of a rotation and its quickness are directly depended on dimension number. For example, in 2 dimensional space, the rotation path is always perpendicular to the course direction before the rotation even for each thin space part. There cannot emerge a soft rotation in 2 dimensional space because of limited and at last fixed speed, as a shortcut in a different direction will not emerge with an angle. To reduce rotational latency and hardness, and thus to reduce emerging centrifugal force because of emerging distance taken length, there must always exist enough number of dimensions. Higher dimen- sional objects do faster displacement. Only infinite dimensional space allows movements to be in any direction of them all together untimely manner without any angle, latency and thus a sensed inertia.

For these reasons, because of its existence which means the area covered, an object has to do a circular motion with an angle by passing all the limited dimensions which are a result of time to be formed since any point of space is intersection point of dimensions as a part of space, even when matter is static which means has no observational outer space motion at that time. Right this point, it can be said that more speed means more dimension scanning. Thereupon, density of an object in emergence space which is the space emerges over absolute space as a virtual part of it in a time interval by repeating the same motion with a frequency during passing the limited dimensions, cannot exist in absolute space, emergence area itself is included as well. It is in a closed and limited area for any selected dimension.

As it can be seen, nothing lived forever during infinite time period since nothing can be distributed as a density upon infinite dimensional space, and it says that any act and phenomenon are included in existence density; they emerge, act and get lost together. To understand this better manner,

$$
\sum_{\mathrm{t}=1}^{\infty} \frac{\mathrm{mc}^{2}}{\frac{4 \pi \mathrm{r}^{3}}{3}}
$$

this operation can be used for $\mathrm{r}=\mathrm{ct}$ when you assume, that the mass has just emerged at a point; because gravity is always active in any direction and also no mass has infinite energy to provide attraction from infinity.

As gravity is always active in all directions in space, for an object which has just emerged with $\mathrm{mc}^{2}$ energy, the energy density where as far as $r$ from the object can only change by $\mathrm{r}=$ ct since the object cannot exist at every point of space untimely manner. All works can be done as much as total energy; so for an object which has energy density equal to its total energy after $t$ time, over $\frac{\mathrm{mc}^{2}}{\frac{4 \pi^{3}}{3}}$ for $\mathrm{r}=\mathrm{ct}$,

$$
\mathrm{mc}^{2}=\frac{3 \mathrm{~m}}{4 \pi \mathrm{c}}\left(\sum_{\mathrm{t}=1}^{\infty} \frac{1}{\mathrm{t}^{3}}\right)
$$

(46) can be written; but according to this equality, the condition must be $\mathrm{t} \leq$; thus being the initial time is $\mathrm{t}_{0}$,

$$
\mathrm{mc}^{2}=\frac{3 \mathrm{~m}}{4 \pi \mathrm{c}}\left(\sum_{\mathrm{t}=\mathrm{t}_{0}}^{1} \frac{1}{\mathrm{t}^{3}}\right)
$$

over (47), for the time intervals which the time interval between each consecutive interval is the same, it can be written as (47a).

$$
\mathrm{s}=\sum_{\mathrm{t}=\mathrm{t}_{0}}^{1} \frac{1}{\mathrm{t}^{3}}=\frac{1}{\left(\frac{\mathrm{k}}{\mathrm{c}}\right)^{3}}+\frac{1}{\left(\frac{2 \mathrm{k}}{\mathrm{c}}\right)^{3}}+\frac{1}{\left(\frac{3 \mathrm{k}}{\mathrm{c}}\right)^{3}}+\ldots+\frac{1}{\left(\frac{\mathrm{ak}}{\mathrm{c}}\right)^{3}}
$$

Here, as a is very big number, it can be ignored; so

$$
\mathrm{s}=\left(\frac{\mathrm{c}}{\mathrm{k}}\right)^{3}\left(1+\frac{1}{2^{3}}+\frac{1}{3^{3}}+\frac{1}{4^{3}}+\ldots+\frac{1}{\mathrm{a}^{3}}\right)
$$

(47c) can be used instead of (47b). 


$$
\mathrm{s}=\left(\frac{\mathrm{c}}{\mathrm{k}}\right)^{3}\left(\sum_{\mathrm{b}=1}^{\infty} \frac{1}{\mathrm{~b}^{3}}\right)
$$

Here, being it is $\zeta(3)=\sum_{\mathrm{b}=1}^{\infty} \frac{1}{\mathrm{~b}^{3}}$, over $\frac{3 \mathrm{~ms}}{4 \pi \mathrm{c}}=\mathrm{mc}^{2}$, it becomes (48).

$$
\mathrm{s}=\frac{4 \pi \mathrm{c}^{3}}{3}
$$

If this is the equation, then over $\frac{4 \pi \mathrm{c}^{3}}{3}=\frac{\zeta(3) \mathrm{c}^{3}}{\mathrm{k}^{3}}$, it becomes (49).

$$
\mathrm{k}=\sqrt[3]{\frac{3 \zeta(3)}{4 \pi}}
$$

As $t_{0}$ is $(\mathrm{k} / \mathrm{c})^{3}$ in $\mathrm{s}$, it becomes (50) the below.

$$
\mathrm{t}_{0}=\frac{3 \zeta(3)}{4 \pi \mathrm{c}^{3}}
$$

Over (46) and thus $\frac{3 \mathrm{~m}_{1} \zeta(3)}{4 \pi \mathrm{c}}=\mathrm{m}_{2} \mathrm{c}^{2}$, it becomes (51),

$$
\frac{\mathrm{m}_{1}}{\mathrm{~m}_{2}}=\frac{1}{\mathrm{t}_{0}}
$$

where $v=\frac{1}{t_{0}}$ as frequency.

$v$ is the maximum natural frequency which space can hold because of the incompressibility property of matter. To have a limit of detected gamma frequencies is not accident according to me as matter has no external supporter energy even if there is no limit theoretically. Matter vibrates as much as this frequency. Matter always uses its total energy which gained during the creation.

$\mathrm{m}_{1} / v$ gets bigger due to the particle number and thus mass which $\mathrm{m}_{1}$ has; but if the particles are handled separately, each one of them is reduced into a threshold mass value. A force is distributing mass on space over time; so after a time, there will only exist information even the mass is still there as distributed extremely as its density in an unit of volume extremely will decrease. Nothing gets lost.

Actually, there is no perpendicularity. Perpendicularity changes over the physical values of the area holds. Namely, you can only get close to perpendicularity; so actually, you can draw extra numerous perpendicular lines to 3 lines which are perpendicular to each other of 3D plane axis, and all of them can be perpendicular to each other; but this happens only over time. Namely, while one of them exists, the other one is not there. Actually it is more suitable to say, that while one of them becomes more certain by extremely getting closer to 90 , the other one becomes uncertain by extremely getting closer to 0 . This extra perpendicular lines emerge due to circular frequency. If frequency increases, then you can detect more of them in a time interval. Namely, a space which has more energy and thus has more frequency is higher dimensional space, and allows objects to move and turn faster.

\section{Warning}

\section{b) Absolute observer requirement}

Handle a fixed object in a limited area in space. A fixed observer does observation in a limited free space which has
$\mathrm{E}_{3}$ energy, and has $\mathrm{E}$ total energy with its all elements. The observer detected the object at $\mathrm{d}_{1}$ length which has $\mathrm{E}_{1}$ total energy without reference; then assume that, $\mathrm{d}_{1}$ length is lengthened to $\mathrm{d}_{2}$ length but the observation angle $\angle \mathrm{BAC}$ does not change. There is a presentation of this event on Fig. 11.

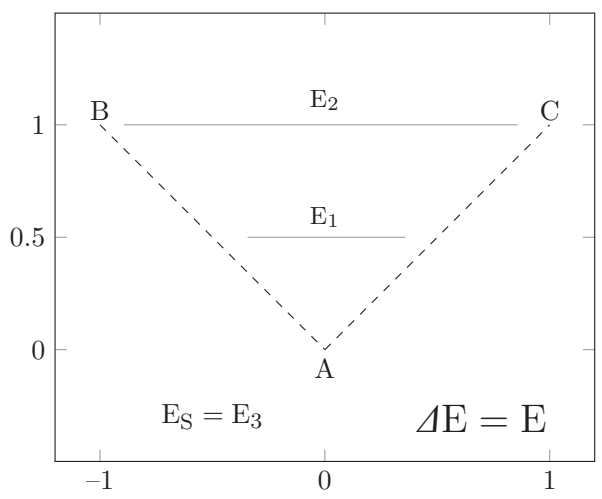

Fig. 11: Change in existence in a limited free space

In this condition, to gain $\mathrm{E}_{2}$ energy of $\mathrm{d}_{2}$ length where $\mathrm{E}_{2}>\mathrm{E}_{1}$, this object has to use free space's energy; therefore at the end of the lengthening, the energy of free space absolutely decreases. If it is assumed that the same operation is done gradually forever, all the energy which the free space has must be used for the observed object that the observer's energy is included as well; hence even if it is assumed that the observation angle does not change, even for the smallest change, as the area covered and its density will change, also the angle has to change; so the equation becomes Eq. (52),

$$
\lim _{\mathrm{n} \rightarrow \infty} \sum \mathrm{E}_{\mathrm{n}}=\mathrm{E}
$$

where $n$ is element number of free space, and $\mathrm{E}$ is total energy of free space with its all elements; so if you increase the energy of the observed object, the observer loses energy; but actually may the observer get lost completely?

If you assume that the space may be able to be bended; but to realize this, more energy is required; because if you want to compare this, handle two different independent space that one of them has $\mathrm{A}$ area has $2 \mathrm{E}$ total energy and $\mathrm{d}=$ $2 \mathrm{E} / \mathrm{Av}^{2}$ density, and the other one has $\mathrm{A} / 2$ area has $\mathrm{E}$ total energy and $\mathrm{d}=2 \mathrm{E} / \mathrm{Av}^{2}$ density which is the same density with the other one. Since $\mathrm{m}$ is dependent of the speed which creates it, it cannot exist such as. As it can be seen for both of them, to create both space, the required energy amount is the same as $\mathrm{E}=\mathrm{dAv}^{2} / 2$; hence, it can be said that more denser space requires more energy; because even if you assume like one of them has X energy, it does not occur such that. For a formation speed of $\mathrm{v}$, if you create more denser space, you must change the work's speed. Force cannot be applied to compress space for the same time. $\mathrm{Ft} / \mathrm{t}_{\mathrm{a}}$ ratio always has to change where $t=1$ second or another reference time which creates the force, and $t_{a}$ is the application time of Ft. This creates different speeds and so creates different space energy as work done is equal to kinetic energy. We can show this phenomenon as Eq. (53),

$$
\frac{\Delta \mathrm{E}}{\Delta \mathrm{E}-\mathrm{E}}>1
$$

where $\Delta \mathrm{E}$ is total energy of the medium and $\mathrm{E}$ is partial energy of any point in this medium.

Hence if you handle a limited space which has a fixed formation speed, if a denser area emerges, as the speed is fixed 
as there is always single work to create all the space and its elements, there will always emerge a resistance which means wish to have more speed to get denser; otherwise this density wants to get lower density and be more ordered; so matter always wants to be distributed; so while $d_{1}$ length is lengthening for a limited energy, space cannot be bended more than enough; so still the angle will change even for the smallest change. Just change amount of the angle for the same time interval will be different relatively to the other if there is more tension; but at a point, it must be recovered by a bigger change relatively to lower stress space.

There cannot be a zero resistance even for before the beginning of time. Matter always wants to be distributed. Denser area is always more disordered, and more energy is required to create denser area.

\section{Inference: Stress}

Hence, even if you increase the energy of the observed object for any magnitude limited formation speed, the observer cannot be lost; because space and matter have an incompressibility feature. At a point, it requires more energy than total energy of free space with its all elements. If you increase the observed object's energy together with total energy of the space by a new formation speed gradually forever being the observer's energy is fixed even for changing formation speed, you must be decrease density of the observer as fixed area cannot lift more than enough density. This is the same with fixed speed space's compress; so if there is no observer, there is no matter. Actually it means if there is no matter, there is no matter; because there is no difference between observation energy and matter. Observation energy is also matter, and has $\mathrm{E} / \mathrm{v}^{2}$ mass for a formation speed. As matter is a virtual part of infinity, as required by infinite frequency, matter has no work ability. Infinity must work instead of any worker; so also observation is done by observation of infinity; hence the right expression is that if there is no absolute observer, there is no matter.

There would no matter if there was no observer. Observer is absolute. The observers which are in a limited uncertain emergence space which has limited formation speed, cannot be lost.

\section{Inference: Eye of God}

Because of the incompressibility feature, it can be said that even for the smallest existent work, work can only be done against recovery wish of space; so matter cannot create itself as a forcing each small time interval of each small time interval is required which brings matter out total energy. Already, matter is uncertain. It means that it must be repeated as a part of infinity. Uncertainty increases or decreases between absolute absence and absoluteness but its loss; so a worker is required for each small time interval instead of any worker. Also as it was said in excessive time section, any motion has to be deterministic. They are created untimely manner. There is no time difference between two information in imaginary time. Anything is element of infinity. If there shall emerge a work, already it must be a part of infinity. Neither anything can be added in nor detracted from infinity. Already this condition requires to be deterministic of any act. If everything is element of infinity, and also if infinity forces matter as there is no alternative to create it as matter is absolute inert because of a required absolute worker instead of any worker in each small time interval, then to be absolute requires to have consciousness. Nothing can be random. The infinity's infinite act is also determined and is included its infinite information. Emerging things are the results.

Uncertain observers may affect matter. To see, please check 'A time concept' section below.

\section{Inference}

\subsubsection{Momentum}

As area had been always existed, that already the above stated time functions as velocity, acceleration and distance taken functions are dependent on this space as well; so as they cannot be separated from time, also they cannot be separated from inertia or latency which is the result of motion.

The latency here I used is due to resistance of free space which matter emerges over it. For a fixed magnitude influence potential that this potential does not have to be space-dependent being created over the same space since it can be God or infinity itself, work can be done at different speeds according to the resistance of the space. If there was no resistance, the existent smallest force would move the existent biggest mass at infinite speed; but it does not happen such. Even if the influence has a potential like this, the space always delays it. For another example, light speed is also limited because of this reason; because light moves from disordered area to ordered area by acceleration during its movement; but it cannot find more ordered space to go faster since disordered points block it.

\section{Warning}

As matter has infinite frequency as a requirement of to be virtual part of absolute space, inertia emergence and its magnitude can only emerge and increase in time as dependent of time in $0 \leq \mathrm{t}<\infty$ second interval; then the equation for latency in $[0, \infty)$ interval becomes Eq. (54)

$$
\frac{\mathrm{L}_{\mathrm{r}}\left(\mathrm{t}_{\mathrm{n}+1}-\mathrm{t}_{\mathrm{n}}\right)}{\mathrm{t}}=\mathrm{L}_{\mathrm{d}}
$$

where $\mathrm{L}_{\mathrm{r}}$ is reference latency, $\mathrm{L}_{\mathrm{d}}$ is detected latency. Even the reference latency has sub-latency because of emerging infinite frequency; so here it must be always $\left(t_{n+1}-t_{n}\right) / t$, and it means that which latency interval is used since there cannot be a motionless interval. It does not mean there is a time slip. It means that there was a potential which changes over time whatever the emergence type; but it changed by an influence and is detected as different during change in t time. Emergence motion moves at that time. The equation will always provide the changing which occurs over time where L can be mass, distance taken, velocity, force or acceleration as elements of motion even as relatively to each other or in itself that if Eq. (54) does not calculate potential change or influence potential change, $\mathrm{L}_{\mathrm{r}}$ and $\mathrm{L}_{\mathrm{d}}$ are always two parts of the same L latency as kind; but if it calculates influence potential which causes potential change or stays as influence potential, then they are different to categorize.

As emergence area and its elements have infinite frequency, mass which is the place that space exists denser there as there cannot be an absolute emptiness because of deceleration from infinity, can only be shown as Kgs which means "Kilogram per second". For a mass which increases in time, it becomes Eq. (55) if it calculates potential change,

$$
\frac{m_{r}\left(t_{n+1}-t_{n}\right)}{t}=m_{d}
$$

where $\left(t_{n+1}-t_{n}\right) / t=a$; so finally it becomes Eq. (56),

$$
\mathbf{F}=-\mathrm{ma}
$$

if it calculates influence potential where $\mathrm{F}$ is influence potential even if it is used or not, $\mathrm{m}$ is potential and $\mathrm{a}=\|-\mathbf{a}\|$. It 
can be transformed into Eq. (56a), over $\mathbf{v}=-\mathbf{a t}$.

$$
\mathbf{F t}=\mathrm{mv}
$$

Here $m v$ is momentum. Momentum means mass relative to time. It is still mass in short. If $\mathrm{m}$ moves here, then $\mathrm{v}$ is the speed of the moving mass. Otherwise already this speed is the speed of mass or force change.

$\mathrm{m}_{\mathrm{r}}$ is detected as $\mathrm{F}$ force or mass during the latency. This is inertia. Any work can only be done in a time interval, and so worker or the thing which is made it worked does work by some periods as active, and does not do work by some periods as inert. Actual result of this is to be absolute inert of matter since an absolute energy has to do work instead of all another uncertain workers even for each small time period. Matter never gains a work potential, and it seems that everything has to be determined before to be created; but still it is calculable like it already had an influence potential like infinite although it is not.

Also latency equality can be shown as Eq. (57)

$$
\mathrm{i}_{\mathrm{e}}\left(\frac{\frac{\mathrm{x}_{2}}{\mathrm{t}_{2}}-\frac{\mathrm{x}_{1}}{\mathrm{t}_{1}}}{\mathrm{t}}\right)=\Delta \mathrm{i}
$$

since space cannot be independent of infinite. There is no absolute emptiness; so if you assume that 1 dimensional space part of x started vibration at a frequency by a natural latency in a time interval as nothing is continuous, emerging inertia $i_{e}$ which is always together with motion even for the smallest time turns into $\Delta \mathrm{i}$ for any time interval during the formation or even during an observational outer space motion which is after the emerging. As the result, mass equation turns into Eq. (56) again.

Also over uncertainty, if $\mathrm{m}_{\mathrm{b}}$ is the assumed basic mass when uncertainty warns, and $\mathrm{m}_{\mathrm{d}}$ is the mass detected at the end of a $t_{r}$ reference time; by the defined elements, emergence of a mass can be expressed as Eq. (58).

$$
\mathrm{m}_{\mathrm{d}}=\mathrm{m}_{\mathrm{b}} \int_{0}^{\mathrm{t}_{\mathrm{r}}} \mathrm{d}_{\mathrm{t}_{\mathrm{r}}}
$$

The same $m_{d}$ mass has to get different magnitudes for any time between $\left[0, t_{r}\right]$ or $\left[t_{r}, 1\right]$ and "Kilogram per second" unit that can be shorten as (Kgs) unit for 1 second.

$$
\mathrm{v}_{\mathrm{f}}=\mathrm{m}_{\mathrm{d}} / \mathrm{m}_{\mathrm{b}} \mathrm{t}_{\mathrm{r}}
$$

Eq. (59) is the change in mass for a formation velocity of $v_{f}$, and it is equal to some variable values according to emergence values; so it can be said that mass is a varying effect which is detected within displacement time.

Mass is variable effect which is detected in displacement time. It is actually magnitude of change in influence potential.

\section{Warning}

As displacement of the mass which moves in outer space after the emerging increases for the reference time of absolute upper limit of 1 second; so also its mass magnitude must increase absolutely; because a motion in outer space cannot be independent of emergence just as emergence cannot be. As matter is always together a motion and so energy, when it moved, then it means formation motion moved; thus mass of the moving objects absolutely increases as matter gains mass by the same way.

For $m_{d}(\mathrm{Kgs})$ mass which had been accelerated after it emerged, as reference time of $t_{r}$ is 1 second and $m_{S}$ is an outer space motion mass, it turns into Eq. (60).

$$
\mathrm{v}=\mathrm{m}_{\mathrm{S}} \mathrm{t} / \mathrm{m}_{\mathrm{d}} \mathrm{t}_{\mathrm{r}}
$$

$\mathrm{m}_{\mathrm{d}}(\mathrm{Kgs})$ mass is perceived as $\mathrm{m}_{\mathrm{s}}(\mathrm{Kgs})$ mass according to the acceleration time $t$ by the reason of inertia. There is no other mass. During the acceleration work, $\mathrm{m}_{\mathrm{d}}$ (Kgs) can be assumed as the basic mass. The velocity here is equal to the velocity of the mass which moves in observational outer space if the mass moves. If the mass does not move, this velocity is already the velocity of the change in the mass over time as a perceived mass. For $\mathrm{m}_{\mathrm{d}}=\mathrm{m}$ to be more clear of it, this condition can be maintained as Eq. (61),

$$
\mathbf{F t}=\mathrm{mv}
$$

as $\mathrm{m}_{\mathrm{S}}(\mathrm{Kgs})$ mass will get its standard mass magnitude if it slows down; thus this mass can be assumed as a force as it is variable, temporary.

Actually, as matter emerges over time, for smaller application time interval than absolute upper time limit which matter gains its maximum mass magnitude value, the mass which the force is applied is smaller than general mass magnitude as the force is smaller as well; so actual equation for this condition is $\mathrm{Ft}^{2}=\mathrm{mtv}$ where $t<t_{u}$ being $t_{u}$ upper limit which means creation time that we accept it as 1 second. Additionally, there is one more option that as motion is deterministic, when you wish to use more energy by the same force by using the force in a smaller time, there will no magnitude difference; thus $\mathrm{Ft}^{2}=\mathrm{mtv}$ becomes invalid. This requires relative time. Time passes different for different motions. Even so, the energy is still used from total energy of universe. If this is the situation, then there are infinite times that is more suitable with my theory since there are uncertainty and time differences. You cannot say which time is the beginning for a part off all mass of the universe. Namely, you cannot catch the times smaller then 1 second since frequency is so high. Even its mass increases when you moved at that time.

Warning

\subsubsection{Kinetic energy}

As kinetic energy, use $\mathrm{x}=\mathrm{vt}$ on $\mathrm{Ft}=\mathrm{mv}$ or use $\mathrm{v}^{2}=\mathrm{ax}$ on $\mathrm{F}=\mathrm{ma}$. They turn into $\mathrm{Fx}=\mathrm{mv}^{2}$ and so into Eq. (62).

$$
\mathrm{W}=\mathrm{mv}^{2}
$$

Also over Eq. (63), it becomes Eq. (62) again,

$$
\mathrm{W}=\int \mathrm{mad}_{\mathrm{x}}
$$

where $\mathrm{a}=\frac{\mathrm{v}}{\mathrm{t}}$ and $\mathrm{x}=\mathrm{vt}$. Energy means mass relative to velocity, and is actual momentum. This is energy; because actually on $\mathbf{F t}=\mathrm{mv}$ equation, for one fixed time or velocity value, the other one of these two time or velocity can take any different value. Right this point, the reference is distance taken.

When a mass moves from A point to B point in space, it means formation motion moved. As mass cannot be independent of speed, the equation becomes Eq. (64); because matter can be used as much as its total energy. All works are as much as total energy and existence of matter; so last moving 
total energy at the last condition of matter as a result must be equal to sum of observational outer space kinetic energy and static total energy.

$$
\mathrm{m}_{0} \mathrm{c}^{2}+\mathrm{mv}^{2}=\mathrm{mc}^{2}
$$

For Eq. (64), change in mass becomes Eq. (65).

$$
\mathrm{m}=\frac{\mathrm{m}_{0}}{1-\frac{\mathrm{v}^{2}}{\mathrm{c}^{2}}}
$$

This mass is the mass which already had been accelerated and is moving at a constant speed of v. For acceleration work, if $\mathrm{p}_{0}=\mathrm{m}_{0} \mathrm{c}$ and $\mathrm{v}=\mathrm{Ft} / \mathrm{m}_{0}$, then for $\int \mathrm{md}_{\mathrm{v}}$, it will be Eq. (66).

$$
\mathrm{m}=\mathrm{p}_{0} \tanh ^{-1}\left(\frac{\mathrm{Ft}}{\mathrm{p}_{0}}\right)
$$

As you can see on Eq. (65) or Eq. (66), the limit is c for v = $\mathrm{Ft} / \mathrm{m}_{0}$ equation; but actually work can be done by any big multiples of $\mathrm{c}$ for $\mathrm{Ft} / \mathrm{m}_{0}$ equation and $\mathrm{Ft} / \mathrm{m}_{0}>\mathrm{c}$ status even if the resulting velocity does not increase so much. We must re-determine the condition. For Eq. (67) the below,

$$
\mathrm{m}_{\mathrm{n}+1}=\mathrm{p}_{\mathrm{n}} \tanh ^{-1}\left(\frac{\left(\mathrm{Ft} / \mathrm{p}_{0}\right)-\left\lfloor\mathrm{Ft} / \mathrm{p}_{0}\right\rfloor}{\mathrm{p}_{\mathrm{n}}}\right)
$$

it will be Eq. (68) for $\mathrm{p}_{\mathrm{n}}=\mathrm{m}_{\mathrm{n}} \mathrm{c}$ as

$$
\mathrm{m}=\sum_{\mathrm{n}=0}^{\left\lfloor\mathrm{Ft} / \mathrm{p}_{0}\right\rfloor} \mathrm{m}_{\mathrm{n}+1}
$$

for the work's whole value as there is no difference for an one-piece work without hand taking or a work in pieces since already the work is done one by one. For Ft $/ \mathrm{m}_{0}<\mathrm{c}$ status, Eq. (66) can be used.

On $\mathrm{Ft}=\mathrm{mv}$ equation, the multiplication of $\mathrm{Ft}$ does not change even if the mass or the velocity on the momentum equation changes by any rule; because they are selfformed according to $\mathrm{Ft}$ work if this the equation; so over $\mathrm{Ft}=\mathrm{mv}_{\mathrm{r}}=\mathrm{m}_{0} \mathrm{v}$ equation, the actual reached velocity $\mathrm{v}_{\mathrm{r}}$ will be Eq. (69).

$$
\mathrm{v}_{\mathrm{r}}=\mathrm{v}\left(1-\frac{\mathrm{v}^{2}}{\mathrm{c}^{2}}\right)
$$

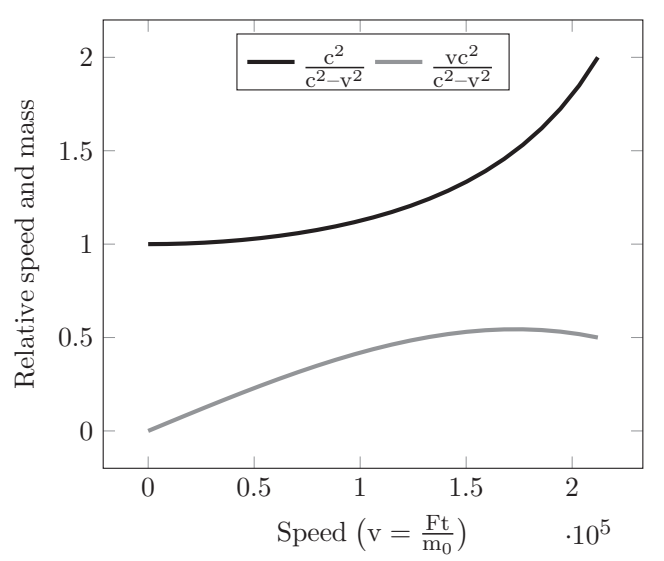

Fig. 12: Mass and speed changing graphic from $\mathrm{m}=\mathrm{m}_{0}$ to $\mathrm{m}=$ $2 \mathrm{~m}_{0}$
For Eq. (65) and Eq. (69), kinetic energy becomes Eq. (70) over $\mathrm{W}=\mathrm{mv}_{\mathrm{r}}^{2}$

$$
\mathrm{W}=\mathrm{m}_{0} \mathrm{v}^{2}\left(1-\frac{\mathrm{v}^{2}}{\mathrm{c}^{2}}\right)
$$

Total energy becomes Eq. (71).

$$
\mathrm{E}=\frac{\mathrm{m}_{0} \mathrm{c}^{2}}{1-\frac{\mathrm{v}^{2}}{\mathrm{c}^{2}}}
$$

As it can be seen over Eq. (71), mass and energy magnitudes are not conserved in focal point even if work done energy is conserved. Even if the total energy of the universe does not change, total energy and mass of the focal points of the universe can change. During this changing, the total density of the universe changes; but its total energy is always conserved.

Mass and energy magnitudes are not conserved in focal points of universe.

Inference

For the above stated equations it becomes Eq. (72) over $\mathrm{E}=\mathrm{W}+\mathrm{m}_{\mathrm{o}} \mathrm{c}^{2}$.

$$
\frac{\mathrm{v}^{2}}{\mathrm{c}^{2}}=0
$$

It means it is always $\mathrm{v}=0$ that it is always $\mathrm{c}$. This is the verifying. Light speed is the highest speed, and the lower is not possible. Matter uses the same space at the same time.

\subsection{Relative motion relations}

There are some special phenomena for relative motions like relative speed and relative size; because an object is always more uncertain at a farther distance than a closer distance size relatively to eye.

\subsubsection{Relative velocity at a perfect distance}

Relative velocity of two moving objects is the displacement of the moving one according to the resultant of the two moving objects when one of them is assumed as fixed. Relative velocity magnitude is the same for the both moving object. The thing which is not the same for each of them is the direction of the movement; because for example, for one of the infinite number relative motion combination of the two object, one of them may see itself as slowing down even that time it is not slowing down. To be positive or negative of velocities according to a reference is related with their directions.

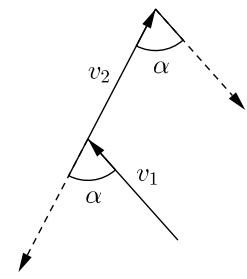

Fig. 13: Relative velocity at a perfect distance

When the velocity of the moving object which is assumed as fixed is subtracted in vector from the other moving object which is not counted as fixed, it is like distance taken in the 
direction of the resultant according to the angle emerging between them as the result of the subtracting. The observer in the other name the moving object which is not counted as fixed observes itself at the resulting speed and in that direction of the resultant as an illusion. This calculation can only be done for the objects which are at a perfect distance relative to each other. This means that when the lengths of the vector straight lines are drawn at the same ratio with the velocities, they are intersected at a point perfect manner like on Fig. 13. Otherwise, a second relative calculation must be done as the objects which are at a far distance cannot be seen or are observed as smaller than the original size, as also the velocity will be observed as static like stars.

For Fig. 13, over the law of cosines, the velocity relative to $\mathrm{v}_{2}$ means detected by $\mathrm{v}_{1}$ as an illusion is Eq. (73) over $\mathrm{v}_{\mathrm{R}}^{2}=\mathrm{v}_{1}^{2}+\mathrm{v}_{2}^{2}-2 \mathrm{v}_{1} \mathrm{v}_{2} \cos \alpha$.

$$
\mathrm{v}_{\mathrm{R}}=\sqrt{\mathrm{v}_{1}^{2}+\mathrm{v}_{2}^{2}-2 \mathrm{v}_{1} \mathrm{v}_{2} \cos \alpha}
$$

For the velocity relative to $\mathrm{v}_{1}$, as it will be $\mathrm{v}_{\mathrm{R}}^{2}=\mathrm{v}_{2}^{2}+\mathrm{v}_{1}^{2}-$ $2 \mathrm{v}_{2} \mathrm{v}_{1} \cos \alpha$, the relative velocity directly will be as Eq. (73) again. The changing thing is relative or absolute destination on space.

\subsubsection{Relative size of fixed objects}

As the size included observations which are different than the above stated one, area of an object is observed as smaller when you moved away from it, and is observed as bigger when gotten closer to it. Any object always can only exist between this two conditions. Right this point, we must comment according to a size reference. Let us assume that this reference is the length reference of the 1 dimensional object has $|\mathrm{BC}|=\mathrm{d}$ length, is as also a standard on Fig. 14. Assume that this standard is to be observed of this $|\mathrm{BC}|$ as $|\mathrm{BC}|$ from the rectangular distance of $\mathrm{A}$ being $|\mathrm{AD}|=|\mathrm{BC}|$. This $1 \mathrm{di}-$ mensional length is enough even for 3D observation; because a $2 \mathrm{D}$ area will occur, and so the same 1 dimensional calculation will be done for both vertical and horizontal components.

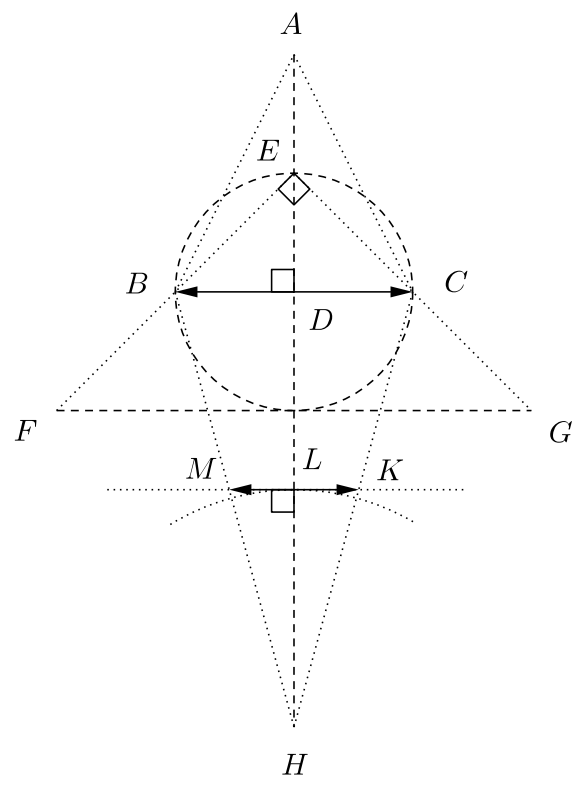

Fig. 14: Perpendicular observation of fixed objects

Right this point, there are 2 different conditions to clarify. They are perpendicular observation and angled observation.

\section{a) Perpendicular observation}

This observation is done on origin of the observed object. If the observer is closer than the determined standard like to be on E point on Fig. 14, the equations which were derived the below can calculate the observed size, and it will be like FG.

\section{First solution:}

As the equation is $|\mathrm{AD}|=|\mathrm{BC}|=|\mathrm{LH}|$, if we use $\Delta \mathrm{HLK}$ triangle which is one of two the same $\triangle \mathrm{HLK}$ and $\triangle \mathrm{HLM}$ triangles, over the Pythagoras Theorem, the equation will be as Eq. (74).

$$
|\mathrm{HK}|^{2}=|\mathrm{HL}|^{2}+|\mathrm{LK}|^{2}
$$

When $|\mathrm{HK}|^{2}$ over Eq. (74) is used on the law of cosine in the same $\triangle \mathrm{HLK}$ triangle for $\angle \mathrm{LHK}=\alpha / 2$, it will be as Eq. (74a).

$$
|\mathrm{HK}|=\frac{|\mathrm{HL}|}{\cos (\alpha / 2)}
$$

If $|\mathrm{HL}|$ over Eq. (74a) is used on Eq. (74) again, it will be as Eq. (75) being $|\mathrm{HL}|=|\mathrm{AB}|=\mathrm{d}$ is the actual size of the object, $|\mathrm{MK}|=\mathrm{d}^{\prime}$ is the observed size of the object.

$$
\mathrm{d}^{\prime}=2 \mathrm{~d} \sqrt{\frac{1}{\cos ^{2}(\alpha / 2)}-1}
$$

\section{Second solution:}

As we know emerging observation angle and standard size of the observed object, we can use one of the half angle formulas. For tangent function, being $\tan (\alpha / 2)=|\mathrm{LK}| /|\mathrm{HL}|$, over the tangent half angle formula, the equation will be Eq. (76),

$$
\tan \alpha=\frac{4 \mathrm{dd}^{\prime}}{4 \mathrm{~d}^{2}-\mathrm{d}^{\prime 2}}
$$

and it can only be solved by solving equation.

\section{Third solution:}

Being $\angle \mathrm{BHC}=\alpha$, it will be Eq. (77) over the law of cosines in $\triangle \mathrm{MHK}$ for $|\mathrm{HM}|=|\mathrm{HK}|$ equation.

$$
\cos \alpha=1-\frac{|\mathrm{MK}|^{2}}{2|\mathrm{HK}|^{2}}
$$

Also it will be Eq. (77a) for the same angle over the law of cosines in $\triangle \mathrm{BHC}$ for $|\mathrm{BH}|=|\mathrm{CH}|$ equation.

$$
\cos \alpha=1-\frac{|\mathrm{BC}|^{2}}{2|\mathrm{CH}|^{2}}
$$

Over the Pythagoras Theorem in $\triangle \mathrm{HDC}$ for $|\mathrm{HC}|=|\mathrm{HB}|$ and $|\mathrm{BD}|=|\mathrm{DC}|=|\mathrm{BC}| / 2=\mathrm{d} / 2$ equations, it will be Eq. (77b).

$$
|\mathrm{HD}|^{2}+|\mathrm{DC}|^{2}=|\mathrm{HC}|^{2}
$$

As the size reference which was determined the above is to be observed of an object at its original size when it is observed from the distance has the same length with the object, then the equations must be $|\mathrm{HL}|=|\mathrm{BC}|=\mathrm{d}$ and $|\mathrm{ML}|=|\mathrm{LK}|=$ $|\mathrm{MK}| / 2$, and so over the Pythagoras Theorem in $\triangle \mathrm{HLK}$, it will be Eq. (77c).

$$
|\mathrm{HL}|^{2}+|\mathrm{LK}|^{2}=|\mathrm{HK}|^{2}
$$


From the similarity between $\triangle \mathrm{HLK}$ and $\triangle \mathrm{HDC}$, the relation is $|\mathrm{HK}| /|\mathrm{HC}|=|\mathrm{LK}| /|\mathrm{DC}|$, and if it is squared, it will be as Eq. (77d).

$$
|\mathrm{HK}|^{2} /|\mathrm{HC}|^{2}=|\mathrm{LK}|^{2} /|\mathrm{DC}|^{2}
$$

If $|\mathrm{HC}|^{2}$ over Eq. (77b) and $|\mathrm{HK}|^{2}$ over Eq. (77c) are used on Eq. (77d), it will be Eq. (77e),

$$
\frac{\frac{\mathrm{d}^{2}}{4}}{\frac{\mathrm{d}^{\prime 2}}{4}}=\frac{\frac{\mathrm{d}^{2}}{4}+|\mathrm{HD}|^{2}}{\frac{\mathrm{d}^{\prime 2}}{4}+\mathrm{d}^{2}}
$$

and finally it will be as Eq. (77f).

$$
|\mathrm{HD}|=\frac{\mathrm{d}^{2}}{\mathrm{~d}^{\prime}}
$$

If $|\mathrm{HC}|^{2}$ over Eq. (77b) is used on Eq. (77a) for $|\mathrm{HC}|=|\mathrm{HB}|$, $|\mathrm{DC}|=\mathrm{d} / 2$ and Eq. (77f) equations, then the function according to observation angle of $\alpha$ becomes Eq. (78).

$$
\mathrm{d}^{\prime}=2 \mathrm{~d} \sqrt{\frac{1-\cos \alpha}{1+\cos \alpha}}
$$

\section{b) Angled observation}

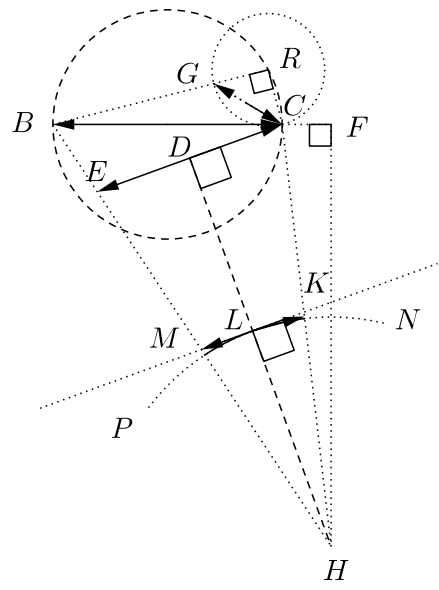

Fig. 15: Angled observation of fixed objects

For angled observations, first of all we must find out perpendicular observation size of the observed object as $|\mathrm{EC}|$ on Fig. 15. To find out it, firstly we must know the distances between the observer and the axises of the observed object. Being $|\mathrm{CF}|=\mathrm{d}_{\mathrm{x}}$ is the distance in $\mathrm{x}$ axis direction, and $|\mathrm{FH}|=\mathrm{d}_{\mathrm{y}}$ is the distance in $\mathrm{y}$ axis direction, $|\mathrm{HC}|$ is as Eq. (79).

$$
|\mathrm{HC}|=\sqrt{\mathrm{d}_{\mathrm{x}}^{2}+\mathrm{d}_{\mathrm{y}}^{2}}
$$

In $\triangle \mathrm{EHC}$, over the law of cosines being $\angle \mathrm{EHF}=\alpha$, it will be Eq. (77b) for $|\mathrm{EC}|=\mathrm{d}^{\prime}$.

$$
|\mathrm{EC}|=2(1-\cos \alpha)\left(\mathrm{d}_{\mathrm{x}}+\mathrm{d}_{\mathrm{y}}\right)
$$

If Eq. (77b) is used on Eq. (78), finally angled observation size of the observed object will be as Eq. (80).

$$
\mathrm{d}^{\prime}=4\left(\mathrm{~d}_{\mathrm{x}}+\mathrm{d}_{\mathrm{y}}\right) \sqrt{\frac{(1-\cos \alpha)^{3}}{1+\cos \alpha}}
$$

There is a special condition for angled observation that the same angle does not mean the same size. An angle can be the same with a perpendicular observation angle or observing angle of an angled observation. The difference is like the difference between $\triangle \mathrm{BRC}$ on Fig. 15 and $\triangle \mathrm{BEC}$ on Fig. 14. They have the same angle; but they cannot detect the fixed size object at the same size. Also like this observation which is done from a distance smaller than the size of the observed object, E point observes the object as $|\mathrm{FG}|$ on Fig. 14. A perpendicular observation reference cannot be determined like $|\mathrm{GC}|$ on Fig. 15.

\subsubsection{Simultaneous relative size change by velocity}

If the objects move, as $\mathrm{d}_{\mathrm{x}}$ and $\mathrm{d}_{\mathrm{y}}$ on Eq. (79) will change in time because of this movement, for the resultant velocity of the two moving objects, that in $\mathrm{x}$ direction, it is $\mathrm{v}_{\mathrm{Rx}}$, and is $\mathrm{v}_{\mathrm{Ry}}$ in y direction, Eq. (80) turns into Eq. (81).

$$
d^{\prime}=4\left(d_{x} \pm v_{R x} t+d_{y} \pm v_{R y} t\right) \sqrt{\frac{(1-\cos \alpha)^{3}}{1+\cos \alpha}}
$$

Here \pm is for direction of the movement. As change in $\alpha$ on Eq. (81), for $\angle \mathrm{BHC}=\alpha$ and $\angle \mathrm{CHF}=\theta$ equations, it will be $\tan (\alpha+\theta)=\left(\mathrm{d}+\mathrm{d}_{\mathrm{x}}\right) / \mathrm{d}_{\mathrm{y}}$ and $\tan (\theta)=\mathrm{d}_{\mathrm{x}} / \mathrm{d}_{\mathrm{y}}$. For these equations, over trigonometric sum formula of tangent, it becomes Eq. (77c),

$$
\alpha=\tan ^{-1}\left(\frac{\mathrm{d}_{\mathrm{y}} \mathrm{d}}{\mathrm{d}_{\mathrm{y}}^{2}+\mathrm{d}_{\mathrm{x}}^{2}+\mathrm{d}_{\mathrm{x}} \mathrm{d}}\right)
$$

and for the movement, it becomes Eq. (81b).

$$
\alpha=\tan ^{-1}\left(\frac{\left(d_{y} \pm v_{R y} t\right) d}{\left.\left(d_{y} \pm v_{R y} t\right)^{2}+d_{(} d_{x} \pm v_{R x} t\right)^{2}+\left(d_{x} \pm v_{R x} t\right) d}\right)
$$

We should add the energy transformations relatively to speed to these calculations as well especially for high speeds. To detect time deviation, also you must use a suitable transformation like the below.

\section{Warning}

\subsection{A time concept}

Time is quite interesting phenomenon in physics, and it seems is relative; but what does it mean to be relative of time? What does to be fixed of light speed mean? Does to be fixed of light speed require observation at light speed? What if we can observe faster than light speed because of increased frame number? Is time effective for this imaginary effect or also time itself is dependent on another actual causative phenomenon? Is it possible to make a wrong conception for time and speed even if the phenomenon we advocate is actually true?

Distribution of image at light speed brings some other phenomena out together with itself. Namely, light speed cannot change even if you take a relative speed like $2 \mathrm{c}$ which is bigger than c. It does not change due to observer, it changes due to observer as illusion. It is always fixed; but each frame of the transfer can change being the light speed or transfer speed is fixed. Namely while you are getting closer to an object which is moving in any direction, you detect it faster than 
its actual speed if you move towards it, and also the observed object gets bigger; but it has an end since the distance between you and it is limited. Namely when you get the closest distance, the relative acceleration converges to zero; because the history of the observed object is stored between you and the object according to the distance between you and the object, and you can watch its history up to $2 \mathrm{x}$ faster whatever the actual direction and speed of the object are, if you can go at speed of light towards the object.

\subsubsection{Placement for transformation}

The below stated formulas are for scalar magnitudes. You must redetermine their marks according to direction of moving bodies.

\section{Warning}

There are 3 points which are on the same line and are going to be used for something for motional transformations on Fig. 16.

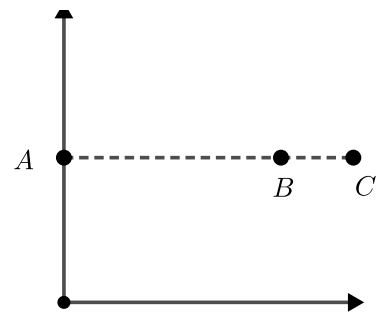

Fig. 16: 3 points in space on the same line

Here we should not intermingle outer space speed of objects and its observational transfer speed. Image transferring speed is light speed and can get any value for different universe being fixed. We accept it as c.

Now think that $\mathrm{A}$ and $\mathrm{C}$ are fixed. Naturally $\mathrm{C}$ observes $\mathrm{A}$ as fixed; but there is a special condition that is valid for any universe, $\mathrm{C}$ observes the old condition of $\mathrm{A}$ in changing times according to the distance between them; because light speed is limited. Even, if $\mathrm{A}$ and the distance between $\mathrm{A}$ and $\mathrm{C}$ are big enough, for example when A has already been disappeared by losing its all energy somehow, $\mathrm{C}$ still observes it since there is a time difference.

\subsubsection{Constant speed motion}

Now think that $\mathrm{C}$ is moving towards $\mathrm{A}$ on the same line being $\mathrm{A}$ is fixed or $\mathrm{A}$ is moving towards $\mathrm{C}$ on the same line being $\mathrm{C}$ is fixed or they both move towards each other. As we always observe image of any object, we can write it as Eq. (82) and Eq. (83).

$$
\begin{aligned}
& \mathrm{v}_{\mathrm{A}}^{\prime}=\left(\frac{\mathrm{c}+\mathrm{v}_{\mathrm{C}}}{\mathrm{c}}\right) \mathrm{v}_{\mathrm{A}} \\
& \mathrm{v}_{\mathrm{C}}^{\prime}=\left(\frac{\mathrm{c}+\mathrm{v}_{\mathrm{A}}}{\mathrm{c}}\right) \mathrm{v}_{\mathrm{C}}
\end{aligned}
$$

Here $\mathrm{v}_{\mathrm{A}}^{\prime}$ and $\mathrm{v}_{\mathrm{C}}^{\prime}$ are observed speed of $\mathrm{A}$ and C. $(\mathrm{c}+\mathrm{v}) / \mathrm{c}$ is a multiplier according to initial speeds of moving objects. As we always observe the history of objects by some frames, this is increase amount of the observed frame number in an unit of time over actual and simultaneous speed of observed object that this speed had gained somehow over $\mathrm{Ft}=\mathrm{mv}$ work or over any function without reference. Here the thing which increases the relative speed of $\mathrm{A}$ is the speed of $\mathrm{C}$ since
$\mathrm{C}$ is going to experience more frame. Also the thing which increases the relative speed of $\mathrm{C}$ is the speed of $\mathrm{A}$ since $\mathrm{A}$ is going to experience more frame as well.

As it can be seen over Eq. (82) and Eq. (83), if there are some objects which the sum of their speed as vector quantity is different than zero, then they experience different times relatively, that is always different than zero since there are time differences as emergence priority.

\section{Inference}

Frame number is partially dependent on and actually is independent of light speed. Whatever the initial velocities of objects are, image is distributed at light speed; so if it is possible to move towards an object faster then light by any multiples of c as nc, you detect the observed object as much more faster due to nc. As long as you get faster, you detect the object faster and faster; but as also you cannot move faster than light, you cannot detect objects faster than a limit. Limit of both frame number and the speed of the object which its image is transferred are naturally dependent on light speed by this way.

Right this point you can ask, that for example while $\mathrm{C}$ is observing $\mathrm{A}$, the initial speed of $\mathrm{C}$ changes the observed object's actual outer speed which emerges according to a global time and gained by $\mathrm{Ft}=\mathrm{mv}$, changes by $\left(\mathrm{c}+\mathrm{v}_{\mathrm{C}}\right) / \mathrm{c}$; but at that time is not $\mathrm{v}_{\mathrm{A}}$ is inclusive to show itself at higher speed by increasing its own initial speed? The answer is exact no; because this is an observation and cannot be realized from two points at the same time. Namely, even if $v_{A}$ increases at that time constantly, the observer detects its acceleration after required time according to its own speed but not more. The observer detects a resulting acceleration whatever happened there. As a result, you cannot include a new ratio by $\left(\mathrm{c}+\mathrm{v}_{\mathrm{A}}\right) / \mathrm{c}$ to the observer formula.

The universe emerges over time since has limited energy. It gains its total energy for 1 second. It means there are time differences for each point of space. Namely each cubic meter has time difference by $1 / \mathrm{V}$ where $\mathrm{V}$ is volume of the universe; so when I said global time, it means when that point has $\mathrm{E}_{1}$ and the other has $\mathrm{E}_{2}$ energy. Namely still each point of space get different time according to this global reference time; but as the amount is too small, we should not include it to these calculations. Time is absolute by these rules. The difference between global time of $t$ and $t^{\prime}$ is to be observational time of $t^{\prime}$. Namely all events occur in 1 second by ignored time differences due to the global time; but you cannot observe all of them as also you cannot be observed. This depends due to the distance between observation elements.

\section{Warning}

Think that $n$ times particle pass from a point at light speed. If point starts to move in the direction of particles at $\mathrm{v}$ speed, to see how many particles are detected for $c+v$, the equation becomes $n(c+v) / c$. Here $n$ can be speed of the observed object, and $\mathrm{v}$ can be speed of the observer. Even if the point has an initial speed, you cannot make a fiction such as if $n$ times particle pass at $\mathrm{c}+\mathrm{v}_{\mathrm{i}}$, then for $\mathrm{c}+\mathrm{v}_{\mathrm{i}}+\mathrm{v}_{2}$, there $\mathrm{n}(\mathrm{c}+$ $\left.\mathrm{v}_{\mathrm{i}}+\mathrm{v}_{2}\right) /\left(\mathrm{c}+\mathrm{v}_{\mathrm{i}}\right)$ times particle are detected. Image transfer speed is always fixed as light speed. You always see each frame at light speed; but as you have an initial speed, you detect it like it increased since the energy collected increases in an unite of time. Namely for initial speed, you must change either $n$ or if you accept $n$ as still fixed, it means the observed object is slower. 
You cannot accept one of the moving bodies as static to calculate speed over the other one of them. This changes everything. Namely, for two bodies move towards each other, you cannot make an addition like $\mathrm{v}_{\mathrm{A}}+\mathrm{v}_{\mathrm{C}}=\mathrm{v}_{\mathrm{A}}^{\prime}=\mathrm{v}_{\mathrm{C}}^{\prime}$ operation. Time passes different for these if they move, and speeds are detected different as relatively. Time does not change in the same ratio. Such an addition can only be made if one of them is really static according to global time.

\section{Warning}

To calculate actual independent global position of objects, first we must fix time which is detected different because of observation speed.

$$
\begin{aligned}
& \mathrm{x}_{\mathrm{A}}^{\prime}(\mathrm{t})=\mathrm{v}_{\mathrm{A}}^{\prime} \mathrm{t} \\
& \mathrm{x}_{\mathrm{C}}^{\prime}(\mathrm{t})=\mathrm{v}_{\mathrm{C}}^{\prime} \mathrm{t}
\end{aligned}
$$

Eq. (84) and Eq. (84) are relative positions. $x_{A}^{\prime}$ is the position which is estimated by $\mathrm{C}$, and also $\mathrm{x}_{\mathrm{C}}^{\prime}$ is the position which is estimated by A over the same global t; so to fix them, we must fix times first over Eq. (86) and Eq. (87).

$$
\begin{aligned}
& \mathrm{x}_{\mathrm{A}}(\mathrm{t})=\mathrm{x}_{\mathrm{A}}^{\prime}(\mathrm{t})+\mathrm{t}\left(\mathrm{v}_{\mathrm{A}}-\mathrm{v}_{\mathrm{A}}^{\prime}\right) \\
& \mathrm{x}_{\mathrm{C}}(\mathrm{t})=\mathrm{x}_{\mathrm{C}}^{\prime}(\mathrm{t})+\mathrm{t}\left(\mathrm{v}_{\mathrm{C}}-\mathrm{v}_{\mathrm{C}}^{\prime}\right)
\end{aligned}
$$

Now let us use more object to analyze if there are some special conditions. If the three points on Fig. 16 are handled together, we can write $\mathrm{x}^{\prime}=\mathrm{x}-\mathrm{v}_{\mathrm{B}} \mathrm{t}$ and $\mathrm{t}^{\prime}=\mathrm{t}$ according to the global time when $\mathrm{A}$ and $\mathrm{C}$ are fixed but $\mathrm{B}$ moves towards to $\mathrm{C}$; but is the formula or only its values change due to observation?

As $\mathrm{A}$ and $\mathrm{C}$ are fixed, they observe $\mathrm{B}$ at its actual speed. There is no time deviation, and thus $\mathrm{x}^{\prime}=\mathrm{x}-\mathrm{v}_{\mathrm{B}} \mathrm{t}$ is valid for $\mathrm{t}^{\prime}=\mathrm{t}$.

If $\mathrm{A}$ and $\mathrm{B}$ move in the direction of $\mathrm{C}$, and $\mathrm{C}$ moves in the opposite direction being all of them at the same speed. For this condition, total observation of B becomes Eq. (88).

$$
2 \mathrm{v}_{\mathrm{B}}=\frac{\left(\mathrm{c}-\mathrm{v}_{\mathrm{A}}\right) \mathrm{v}_{\mathrm{B}}}{\mathrm{c}}+\frac{\left(\mathrm{c}+\mathrm{v}_{\mathrm{C}}\right) \mathrm{v}_{\mathrm{B}}}{\mathrm{c}}
$$

It means even if the observation changes due to observer, actually there is no real deviation or delay. This happens for only partial observers which cannot detect all motions at the same time. It signs to a global time. Time is conserved.

\subsubsection{Acceleration motion}

If we apply the above stated rules to acceleration motion, it becomes Eq. (89) and Eq. (90) over Eq. (82) and Eq. (82),

$$
\begin{aligned}
& a_{A}^{\prime}=\left(\frac{c+a_{C} t}{c}\right) a_{A} \\
& a_{C}^{\prime}=\left(\frac{c+a_{A} t}{c}\right) a_{C}
\end{aligned}
$$

where $t$ is global time. For these values, to calculate actual independent global position of objects, again firstly we must fix time which is detected different because of observation speed.

$$
\begin{aligned}
& \mathrm{x}_{\mathrm{A}}^{\prime}(\mathrm{t})=\mathrm{a}_{\mathrm{A}}^{\prime} \mathrm{t}^{2} \\
& \mathrm{x}_{\mathrm{C}}^{\prime}(\mathrm{t})=\mathrm{a}_{\mathrm{C}}^{\prime} \mathrm{t}^{2}
\end{aligned}
$$

Eq. (91) and Eq. (92) are relative positions. $x_{A}^{\prime}$ is the position which is estimated by $\mathrm{C}$, and also $\mathrm{x}_{\mathrm{C}}^{\prime}$ is the position which is estimated by A over the same global t; so to fix them, we must fix times first over Eq. (93) and Eq. (94).

$$
\begin{aligned}
& \mathrm{x}_{\mathrm{A}}(\mathrm{t})=\mathrm{x}_{\mathrm{A}}^{\prime}(\mathrm{t})+\mathrm{t}^{2}\left(\mathrm{a}_{\mathrm{A}}-\mathrm{a}_{\mathrm{A}}^{\prime}\right) \\
& \mathrm{x}_{\mathrm{C}}(\mathrm{t})=\mathrm{x}_{\mathrm{C}}^{\prime}(\mathrm{t})+\mathrm{t}^{2}\left(\mathrm{a}_{\mathrm{C}}-\mathrm{a}_{\mathrm{C}}^{\prime}\right)
\end{aligned}
$$

Let us analyze the acceleration condition somehow.

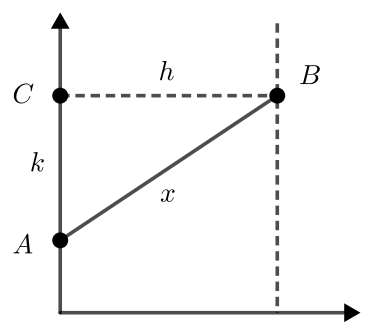

Fig. 17: Satellite

Here on Fig. 17 there is a satellite at $\mathrm{B}$ point moves on the parallel line to $\mathrm{y}$ axis. $\mathrm{CB}=\mathrm{h}$ is the height, $\mathrm{C}$ is a point which its coordinate is known, $\mathrm{A}$ is an object which moves on the $\mathrm{y}$ axis. $\mathrm{AB}=\mathrm{x}$ is the distance between the object and the satellite.

Now assume that $B$ moves in the direction of $+y$ axis and A moves in the direction of $-y$ axis. Namely time slows down for the both relatively and they detect each other as slowing down since they move away from each other, and thus frame number is going to decrease for an unite of time. $\mathrm{k}$ is dependent on the velocities of both A and B; so it becomes Eq. (95)

$$
\mathrm{k}=\left(\mathrm{v}_{\mathrm{A}}+\mathrm{v}_{\mathrm{B}}\right) \mathrm{t}
$$

The time here must be the same time, and is the time of acceleration if the speed is not fixed. Also it starts after a calibration for the current values at $\mathrm{t}_{0}=0$ moment and $\mathrm{k}=0$ point. $\mathrm{x}$ becomes for this as

$$
\mathrm{x}=\sqrt{\left(\mathrm{v}_{\mathrm{A}}+\mathrm{v}_{\mathrm{B}}\right)^{2} \mathrm{t}^{2}+\mathrm{h}^{2}}
$$

Now the condition has been turned into the condition of a linear motion over linear $\mathrm{x}$ distance; but the change emerges over the rule of $\mathrm{x}$ as Eq. (96). It means the motion is like acceleration even for the fixed speeds; so it can written as $\mathrm{x}=\mathrm{at}^{2}=\mathrm{a}_{\mathrm{A}} \mathrm{t}^{2}+\mathrm{a}_{\mathrm{B}} \mathrm{t}^{2}$ and thus over this as $\mathrm{x} / \mathrm{t}^{2}=\mathrm{a}=$ $\mathrm{a}_{\mathrm{A}}+\mathrm{a}_{\mathrm{B}}$ since is not observation yet, and is due to global time as independent. Hence it becomes Eq. (97) over Eq. (89) or Eq. (90),

$$
\mathrm{a}_{\mathrm{A}}^{\prime}=\left(\frac{\mathrm{c}+\mathrm{a}_{\mathrm{B}} \mathrm{t}}{\mathrm{c}}\right)\left(\mathrm{a}-\mathrm{a}_{\mathrm{B}}\right)
$$

where $\mathrm{a}-\mathrm{a}_{\mathrm{B}}=\mathrm{a}_{\mathrm{A}}$ or becomes Eq. (98),

$$
\mathrm{a}_{\mathrm{A}}^{\prime}=\left(\frac{\mathrm{c}+\left(\mathrm{a}-\mathrm{a}_{\mathrm{A}}\right)}{\mathrm{c}}\right)\left(\mathrm{a}_{\mathrm{A}}\right)
$$

where $\mathrm{a}-\mathrm{a}_{\mathrm{B}}=\mathrm{a}_{\mathrm{A}}$ over the same Eq. (89) or Eq. (90). If they are equalized to each other, it becomes Eq. (99),

$$
\mathrm{a}=\frac{\mathrm{a}_{\mathrm{B}}^{2}(1-\mathrm{t})+2 \mathrm{a}_{\mathrm{B}} \mathrm{c}}{2 \mathrm{c}}
$$


and over Eq. (99) it becomes Eq. (100) where $a=a_{A}+a_{B}$.

$$
\mathrm{a}_{\mathrm{A}}=\frac{\mathrm{a}_{\mathrm{B}}^{2}(1-\mathrm{t})}{2 \mathrm{c}}
$$

As we know a over Eq. (96) as $\mathrm{x} / \mathrm{t}^{2}$, we can find $\mathrm{a}_{\mathrm{B}}$ over Eq. (99) and then $a_{A}$ over Eq. (100). Hence, we can find the deviation or actual positions over Eq. (89), Eq. (90) and Eq. (93), Eq. (94).

It seems that time is relative; but actually this is not against to be absolute of time. There is a single time which is creation time of any bodies in the universe for 1 second. The relativistic time emerges because of distance and image transfer speed. We always observe the history of objects in changing times due to distance; but actually this does not mean a time travel or staying as a young person is possible since is only relativistic effect. Age, entropy, total energy etc. are dependent on global time.

Warning

\subsubsection{Energy transformation}

As stated the above, formation motion in the other name the motion which emerges to create matter and outer space observational motion are accepted together. There is no other motion. When mass moves from $\mathrm{A}$ to $\mathrm{B}$ in space, it means creation motion which is because of its existence moves from A to B. Matter uses the same space at the same time for any motion and work. For this condition, assume that kinetic energy is dependent of an operator like $\mathrm{Nmv}^{2}$ that $\mathrm{N}$ can be a rational number as also can be an integer. Now total kinetic energy which matter has for its existence as $\mathrm{Nmc}^{2}$ must be equal to the sume of observational kinetic energy $\mathrm{Nmv}^{2}$ and static kinetic energy $\mathrm{Nm}_{0} \mathrm{c}^{2}$ in the other name static total energy before gaining observational kinetic energy. For this definition, it becomes Eq. (101).

$$
\mathrm{Nm}_{0} \mathrm{c}^{2}+\mathrm{Nmv}^{2}=\mathrm{Nmc}^{2}
$$

Over this the above stated energy transformations occur.

For these energy transformations, the situation becomes more interesting; because Eq. (82) and Eq. (83) must turn into Eq. (102) and Eq. (103) over the energy transformation.

$$
\begin{aligned}
& \mathrm{v}_{\mathrm{A}}^{\prime}=\left(1+\frac{\mathrm{v}_{\mathrm{C}} \mathrm{c}^{2}-\mathrm{v}_{\mathrm{C}}^{3}}{\mathrm{c}^{3}}\right)\left(1-\frac{\mathrm{v}_{\mathrm{A}}^{2}}{\mathrm{c}^{2}}\right) \mathrm{v}_{\mathrm{A}} \\
& \mathrm{v}_{\mathrm{C}}^{\prime}=\left(1+\frac{\mathrm{v}_{\mathrm{A}} \mathrm{c}^{2}-\mathrm{v}_{\mathrm{A}}^{3}}{\mathrm{c}^{3}}\right)\left(1-\frac{\mathrm{v}_{\mathrm{C}}^{2}}{\mathrm{c}^{2}}\right) \mathrm{v}_{\mathrm{C}}
\end{aligned}
$$

For this final condition when you assume that they move towards each other at the same speed, a table emerges like the below.

Table 1: Observational speed change of objects over relativistic energy transformations

\begin{tabular}{c|c|c|c|c}
$\mathrm{v}_{\mathrm{A}}$ & $\mathrm{c} / 4$ & $\mathrm{c} / 3$ & $\mathrm{c} / 2$ & $3 \mathrm{c} / 4$ \\
\hline $\mathrm{v}_{\mathrm{A}}^{\prime}$ & $0.290 \mathrm{c}$ & $0.384 \mathrm{c}$ & $0.516 \mathrm{c}$ & $0.436 \mathrm{c}$
\end{tabular}

The results are quite interesting; because as long as you get faster, you detect the objects slower; because when you get closer light speed during observational motion not creation that already creation is at light speed, observational ability decreases; because you create an imaginary history in the environment between objects since you get closer to image transfer speed. Your appearance time to existence and image transfer time close to each other since there is a single motion for creation and other space motions; but it is more interesting, after $\mathrm{c} / 2$ in the table, again observational speed decreases. The following is the graph of the same rule.

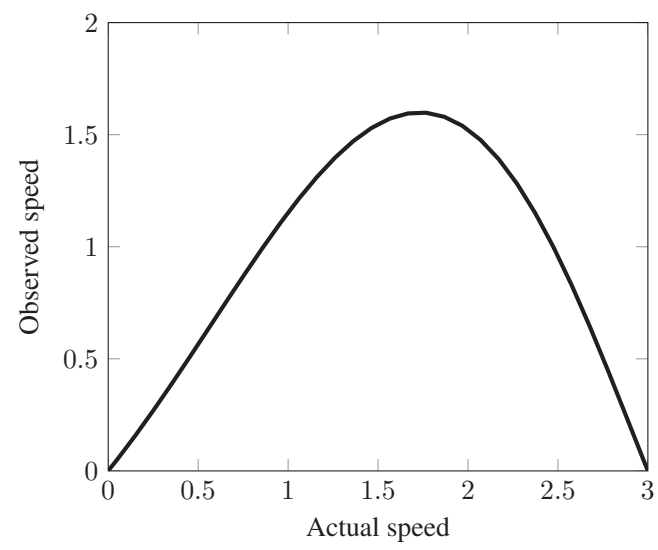

Fig. 18: Observational speed over actual speed where $x=c / 10^{8}$ and the speeds are the same

When you assume that they move towards each other being the observer $\mathrm{v}_{\mathrm{C}}$ has $1 \mathrm{~m} / \mathrm{s}$ speed and the other one has changing speeds, a graph emerges like the below.

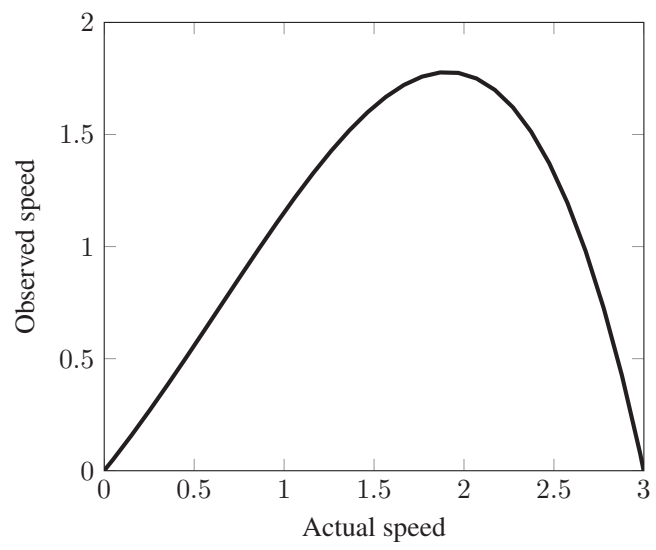

Fig. 19: Observational speed over actual speed where $x=c / 10^{8}$ and the speed of the observer is fixed

In the same manner it is interesting, after c/2 in the table, again observational speed decreases that actually it is not related with $\mathrm{c} / 2$ since also starts decrease before it. It seems, always we must accept total speed of the objects as c.

Table 2: Another table for almost static objects

\begin{tabular}{c|c|c|c|c}
$\mathrm{v}_{\mathrm{A}}$ & $\mathrm{c} / 4$ & $\mathrm{c} / 3$ & $\mathrm{c} / 2$ & $3 \mathrm{c} / 4$ \\
\hline $\mathrm{v}_{\mathrm{A}}^{\prime}$ & $0.234 \mathrm{c}$ & $0.296 \mathrm{c}$ & $0.375 \mathrm{c}$ & 0.328
\end{tabular}




\section{Formation of matter}

\subsection{Mass emergence and area force}

As required by emerging uncertainty which actually is equivalent of Heisenberg's Uncertainty Principle, there cannot exist an absolute part of matter. There must always be infinite frequency which renders matter as an illusion, and does not bring infinite work potential out for matter as kinetic energy is equal to work done. Existence can only exist either as absolute or uncertain which is part of absolute. As matter is uncertain emerges over absolute space, for this condition, force cannot be applied without area. Force is only defined when it is related with application area. Emerging variable values of $\mathbf{P}=\mathbf{F}$ A equation which relates force and area, increase or decrease between absolute absence and absoluteness but their losses. In the same manner, distributed load is mass or force effect as well.

Force cannot be applied to the surface which has no area covered. $\mathbf{F A}=\mathbf{P}$ equation which is the relation between force and pressure, is always valid.

\section{Inference}

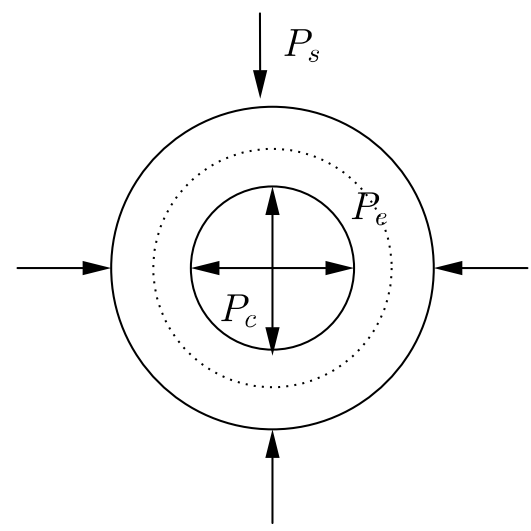

Fig. 20: Mass emergence

Handle an imaginary area on horizon like on Fig. 20 on absolute space, and assume that it expanded. When it expanded, the pressure of the outer space must increase as inner density will decrease since the density of absolute space is fixed. At this condition,

$$
\mathrm{W}_{\mathrm{e}}=\left(\mathrm{P}_{\mathrm{s}}-\mathrm{P}_{\mathrm{e}}\right) \mathrm{x}_{\mathrm{e}} / \mathrm{A}_{\mathrm{e}}=\mathrm{m}_{\mathrm{e}} \mathrm{v}_{\mathrm{e}}^{2}
$$

is the work done where $\mathrm{P}_{\mathrm{S}}$ is the outer space pressure, $\mathrm{P}_{\mathrm{e}}$ is the inner pressure of the expansion, $\mathrm{x}_{\mathrm{e}}$ is the distance taken, $\mathrm{A}_{\mathrm{e}}$ is the surface area of the expansion volume, $\mathrm{v}_{\mathrm{e}}$ is the velocity of the work, and $\mathrm{m}_{\mathrm{e}}$ is the emerging mass as the result of this action in the volume.

When it collapsed, the work done becomes

$$
\mathrm{W}_{\mathrm{c}}=\left(\mathrm{P}_{\mathrm{c}}-\mathrm{P}_{\mathrm{s}}\right) \mathrm{x}_{\mathrm{c}} / \mathrm{A}_{\mathrm{c}}=\mathrm{m}_{\mathrm{c}} \mathrm{v}_{\mathrm{c}}^{2}
$$

where $\mathrm{P}_{\mathrm{S}}$ is the outer space pressure, $\mathrm{P}_{\mathrm{c}}$ is the inner pressure of the collapse, $\mathrm{x}_{\mathrm{c}}$ is the distance taken, $\mathrm{A}_{\mathrm{e}}$ is the surface area of the collapse volume, $\mathrm{v}_{\mathrm{c}}$ is the velocity of the work, and $\mathrm{m}_{\mathrm{C}}$ is the emerging mass as the result of this action in the volume.

For the both conditions, emerging mass as the result is dependent of pressure increases for collapse since $\mathbf{F}$ from $\mathbf{F A}=\mathbf{P}$ increases even if the pressure is in reverse direction, and decreases for expansion because of the same reason. Whatever the direction, if there is an opposite work, the work done is the work done against this opposite work. As the result, it can be said that if mass will emerge, it should be created by a collapse. Whatever which one was used to create it, as constant speed movement is not possible and as the work cannot be done timeless, also the work is done one by one at a frequency. For this reason, any point of the emerging space and mass have different priority since they are the result of the action and are dependent of the work done; so they have different mass magnitudes even the difference can be ignored because of its small magnitude.

Again for the both conditions, as the new emerging area has time difference at any point, when a force is applied to a mass, the area which the force was applied on the mass changes, as the emerging area of the force will change as well; so a linear motion is not possible since an angle must emerge during the work as you cannot determine a middle point. It always slides, and this causes a type of circular motion that actually causes a motion closes a curve as there is no alternative.

As motion, it always emerges in one direction as a resultant; because it can be counted that, on a mass, there are infinite number of forces which create motion in different directions; so as a resultant, motion only emerges in one direction since there cannot be an absolute zero resultant because of emerging infinite time differences and to be latency of mass by a limited speed. Motion is not independent of mass, as mass cannot be. A mass which has no velocity cannot exist. As motion emerges in one direction, whatever losing and emerging frequency of space, and even whatever in how many direction the force was applied, as a result of the action, the resultant space force or mc momentum for a limited and fixed speed since limited universe must have according to work done, must be alone by an angle emerging between the movement direction of the emerging mass and the opposite space force.

Hence it can be said, that an uncertain matter always does a circular motion as there is no alternative. Already, for a limited universe, speed can be counted as fixed; so for a fixed speed, different mass magnitudes are only possible by a circular motion; because different masses means different displacements, and thus means different speeds. If radius increases, then for the same velocity, mass decreases as detected mass in an unit of volume will decrease. The actual reason of the circular motion is to be virtual part of matter; because anything can neither be added in nor be detracted from infinity, as anything is element of infinity. Thereupon, the angle which must emerge between force and mass which is aimed to create also emerges one by one. Force cannot be applied from random points. It has to have an order since force and mass are together in $\mathbf{F}=-$ ma equation, and space emerges one by one in a priority order. Mass can only emerge over space, by using space. Mass turns into space, space turns into mass as a result of the deceleration from infinite, as there cannot be an absolute emptiness.

Emergence is directly depended on $\mathbf{F}=-$ ma equation; so space cannot apply force from random points to form mass. Force is there where mass exists, even if there will emerge an angle.

Inference

\subsubsection{Resultant force}

If area is conservative then for 2 dimensional medium, it becomes Eq. (106),

$$
\mathrm{dxdy}=0
$$

where $\mathrm{ds}=\mathrm{dx}+\mathrm{dy}, \mathrm{ds}^{2}=\mathrm{dx}^{2}+\mathrm{dy}^{2}$ and thus $\mathrm{ds}=\mathrm{ds}^{2}$ and $\mathrm{ds}=1$. Here, even if the two components always change, sum of them is always conserved for any physical value for 
example as $\mathrm{p}=\mathrm{p}_{\mathrm{x}}+\mathrm{p}_{\mathrm{y}}$ and $\mathrm{p}^{2}=\mathrm{p}_{\mathrm{x}}^{2}+\mathrm{p}_{\mathrm{y}}^{2}$. It means 2D motion is not possible that was proven at the beginning of the article over impossibility of perpendicularity.

There are some different possible motion types which have different $3 \mathrm{D}$ motion resultants. To determine which one is possible, again conservation of mass and energy must be used.

As it can be seen over the above stated conditions, if a mass emerges in 3D, first of all, force and speed always must be fixed as the result of finite work done to create universe. A fixed force is applied, and then space gets shape in itself somehow. Even if force changes over time, the rule will be the same as each decrease interval will be assumed as fixed; therefore the equation is always $\mathbf{F}=$ ma. If this is the condition, then it can be said that motion can only emerge if $\mathrm{ds}=\mathrm{dx}+\mathrm{dy}+\mathrm{dz}$ equation is provided; because there is no external mass. It is like it that mass itself creates itself; so $\mathrm{mc}=\mathrm{m}_{\mathrm{x}} \mathrm{c}+\mathrm{m}_{\mathrm{y}} \mathrm{c}+\mathrm{m}_{\mathrm{z}} \mathrm{c}$ equation is always provided. Here, the three component always change; but sum of them is always conserved; therefore the equation becomes Eq. (107),

$$
\mathrm{dx}\left(\frac{1}{\mathrm{dy}}+\frac{1}{\mathrm{dz}}\right)=-\frac{1}{2}
$$

where $\mathrm{ds}^{2}=\mathrm{dx}^{2}+\mathrm{dy}^{2}+\mathrm{dz}^{2}$ and $\mathrm{ds}=\mathrm{dx}+\mathrm{dy}+\mathrm{dz}$.

There are five possibilities for motion's emergence type constantly or partially by some intervals as $\mathrm{dx}=\mathrm{dy}=\mathrm{dz}$, $\mathrm{dx}=\mathrm{dy}, \mathrm{dx}=\mathrm{dz}$ and $\mathrm{dy}=\mathrm{dz}$ or none of them. $\mathrm{dx}=\mathrm{dy}=$ $\mathrm{dz}$ equation is not possible when it is checked over Eq. (107). For the others, if dx is taken from Eq. (107), and then if it is put on its place on mass conservation equation which is $\mathrm{ds}=\mathrm{dx}+\mathrm{dy}+\mathrm{dz}$, the equation becomes Eq. (107a).

$$
\mathrm{ds}=\mathrm{dy}+\mathrm{dz}-\frac{\mathrm{dy} \cdot \mathrm{dz}}{2(\mathrm{dy}+\mathrm{dz})}
$$

Here, assume that it is $\mathrm{ds}^{2}=\mathrm{dx}^{2}+\mathrm{dy}^{2}$ which is the projection of the same ds during forming a sphere by $\mathrm{ds}^{2}=$ $\mathrm{dy}^{2}+\mathrm{dz}^{2}$, where $\mathrm{dx}=\mathrm{dz}$ by the same angle vertically and horizontally according to a fixed reference; then Eq. (107a) becomes Eq. (107b).

$$
\mathrm{dx}^{2}=\mathrm{dy} \cdot \mathrm{dz}+\mathrm{dz}^{2}-\frac{\mathrm{dy}^{2} \cdot \mathrm{dz}^{2}}{4(\mathrm{dy}+\mathrm{dz})^{2}}
$$

Over Eq. (107b), it can be said that $d x=d y$ and $d x=d z$ are not possible. For $\mathrm{dy}=\mathrm{dz}$ equation, it becomes $\mathrm{dx} / \mathrm{dy}=$ $33 / 16$; but if it is used on the main function Eq. (107), it seems that even it is not possible as well; therefore there is only one possibility left that none of them emerges even for any interval of motion, and the components are always different. They never intersect for any combination. Actually it is very compatible for the theory; because any point of free space has emergence priority due to the time differences. As it was said while explaining dimensional density, nothing lived forever that it can be seen over mass density in the same way with dimensional density; so even for after the comma which means in small amounts, between two different times, there must be an evaporation or in the other name decrease in vibration. Motion cannot take infinite distance. The distance converges to a value even if it continues.

As it was said, matter is uncertain; but as area is conservative in accordance with the proven impossibility of perpendicularity over the right triangle at the beginning of the paper, derivative resultant always becomes ds $=1$ for any high dimension number due to $\mathrm{ds}^{2}=\mathrm{ds}$; so for example it is always mc $=1$ as a certain number; but as emerging matter has time differences it becomes Eq. (108),

$$
\frac{1}{1-\mathrm{t}_{0}}>1
$$

where $t_{0}$ is relative time of any selected space point according to any other point being $1 \mathrm{sec}$. is total time of the entire universe or as relatively is the time of another point when the others have not get it. It creates an uncertainty. The creator of the universe can only know the certainty. The inner elements cannot know energy or time being certain. Each physical property that time is included as well, exactly converges to a value being having different length decimals after the comma for any point of the universe continuously. By this way information is conserved forever since there cannot be a flexible collision or friction by the same natural numbers, integers or for example the irrational numbers with a limit as real numbers. Hence even if total physical values of the universe is certain, it is assumed as uncertain by these rules. Otherwise infinite frequency in a limited area renders impossible to emerge of matter being created.

\section{Warning}

As the relation of the components with ds separately and relatively to $\alpha$, over Eq. (107), it becomes Eq. (109),

$$
\mathrm{dz}=-\frac{2 \tan (\alpha) \mathrm{dx}}{2+\tan (\alpha)}
$$

where $\mathrm{dy}=\mathrm{dx} \tan (\alpha)$. If Eq. (109) is used on $\mathrm{ds}=\mathrm{dx}+$ $\mathrm{dy}+\mathrm{dz}$ which is conservation of mass equation, the relation between ds and dx becomes Eq. (109a),

$$
\mathrm{ds}=\mathrm{dx}\left(1+\tan (\alpha)-\frac{2 \tan (\alpha)}{2+\tan (\alpha)}\right)
$$

where $\mathrm{dy}=\mathrm{dx} \tan (\alpha)$. By the same way, the relation between ds and dy becomes Eq. (109b),

$$
\mathrm{ds}=\mathrm{dy}\left(1+\frac{1}{\tan (\alpha)}-\frac{2}{2+\tan (\alpha)}\right)
$$

where $d z=-\frac{2 d y}{2+\tan (\alpha)}$. If $d x$ and $d y$ from Eq. (109a) and Eq. (109b) are used on $\mathrm{ds}=\mathrm{dx}+\mathrm{dy}+\mathrm{dz}$ equation, $\mathrm{dz}$ becomes Eq. (109c),

$$
\mathrm{dz}=1-\frac{1}{\mathrm{M}_{1}}-\frac{1}{\mathrm{M}_{2}}
$$

where $\mathrm{M}_{1}=\left(1+\tan (\alpha)-\frac{2 \tan (\alpha)}{2+\tan (\alpha)}\right)$ and $\mathrm{M}_{2}=\left(1+\frac{1}{\tan (\alpha)}-\right.$ $\left.\frac{2}{2+\tan (\alpha)}\right)$.

Hence even if an assumed point mass spins around a perfect sphere, it turns by different angles vertically and horizontally according to a fixed reference. It requires different speeds or fixed angle by fixed components. As different speeds are not possible as there is only 1 work to create all the universe, it means that there are fixed angles and fixed components; so the equation becomes Eq. (110) over Eq. (107a),

$$
\mathrm{dz}=-\frac{2 \mathrm{ds}_{2} \cos (\alpha) \sin (\alpha)}{2 \cos (\alpha)+\sin (\alpha)}
$$

where $\mathrm{dx}=\mathrm{ds}_{2} \cos (\alpha), \mathrm{dy}=\mathrm{ds}_{2} \sin (\alpha), \mathrm{ds}=\mathrm{dx}+\mathrm{dy}+\mathrm{dz}$, 
and so $\mathrm{dz}=-\frac{2 \mathrm{dx} \cdot \mathrm{dy}}{2 \mathrm{dx}+\mathrm{dy}}$. As the equation is $\mathrm{dz}=\mathrm{ds}_{2} \tan (\alpha+\theta)$, Eq. (110) becomes Eq. (110a).

$$
\tan (\alpha+\theta)=-\frac{2 \cos (\alpha) \sin (\alpha)}{2 \cos (\alpha)+\sin (\alpha)}
$$

Hence, the equation of the angles becomes Eq. (110b) over Eq. (110a) in radiant,

$$
\theta=\pi \mathrm{n}-\alpha-\tan ^{-1}\left(\frac{2 \cos (\alpha) \sin (\alpha)}{2 \cos (\alpha)+\sin (\alpha)}\right)
$$

where $\mathrm{n} \in \mathbb{Z}$. By the other solution for $-\cot (\alpha+\theta)=$ $\frac{1}{2 \cos (\alpha)}+\frac{1}{\sin (\alpha)}$ which is derived over Eq. (110a), $\theta$ becomes Eq. (110c) in radiant,

$$
\theta=\pi \mathrm{n}-\alpha+\cot ^{-1}\left(\frac{-2 \csc (\alpha)-\sec (\alpha)}{2}\right)
$$

where $n \in \mathbb{Z}$, $\cot ^{-1}$ is the inverse cotangent function. For both solutions, the angles are not variable as the angles must be always fixed; so $n$ integer is the same for both of them.

In the same manner, if Eq. (110) is put on $\mathrm{ds}=\mathrm{dx}+$ $d y+d z$ equation which is conservation of mass equation, the equation becomes

$$
0=\sqrt{1+\mathrm{K}^{2}}+\mathrm{K}-\sin (\alpha)-\cos (\alpha)
$$

where $\mathrm{K}=-\frac{2 \cos (\alpha) \sin (\alpha)}{\sin (\alpha)+2 \cos (\alpha)}, \mathrm{ds}=\sqrt{\mathrm{ds}_{2}^{2}+\mathrm{dz}^{2}}, \mathrm{dx}=$ $\mathrm{ds}_{2} \cos (\alpha)$ and $\mathrm{dy}=\mathrm{ds}_{2} \sin (\alpha)$. The roots of Eq. (111) are as follows in radiant,

$$
\begin{gathered}
\alpha=2 \pi \mathrm{n} \\
\alpha=\frac{1}{2}(4 \pi \mathrm{n}+\pi) \\
\alpha=2 \pi \mathrm{n}+2 \tan ^{-1}(2)
\end{gathered}
$$

where $n \in \mathbb{Z}$. These roots cannot be equalized to each other as the result shall be undefined.

Hence if there is a circular motion which closes a curve, that does not mean draws a perfect circle, motion only can emerge by this way as there is no alternative, and a space wave is always perpendicular to emerging mass as resultant mass or velocity always has two perpendicular components according to a reference axis. It means, that force and velocity are perpendicular to each other; because actually force never changes. Force is applied to a part of absolute space, and then space gets shape by emerging frequency, according to work done. There is only 1 and the same work for the entire universe. If force increases, it will be fixed at last, and then frequency will increase. Moving thing is mass always; but as force and mass are together, you can assume that force moved as well.

If the roots are multiplied by each other, the angles become as follows in radiant.

$$
\begin{array}{r}
\alpha=(4 \mathrm{n}+1)\left(2 \pi^{3} \mathrm{n}^{2}+2 \pi^{2} \mathrm{n} \tan ^{-1}(2)\right) \\
\beta=\alpha+\theta=\pi \mathrm{n}-\tan ^{-1}\left(\frac{2 \cos (\alpha) \sin (\alpha)}{2 \cos (\alpha)+\sin (\alpha)}\right)
\end{array}
$$

All right; but here, as the parametric function of the motion, for $\mathrm{t}=\alpha, \alpha$ has no integer solution but $\mathrm{n}=0$; so $\alpha$ is able to get only both of Eq. (114a) and Eq. (114b) angles in radiant over Eq. (111b) and Eq. (111c), and the angle becomes
Eq. (114) in radiant if they are multiplied by each other.

$$
\begin{gathered}
\alpha=\pi \tan ^{-1}(2) \\
\alpha_{1}=\frac{\pi}{2} \\
\alpha_{2}=2 \tan ^{-1}(2)
\end{gathered}
$$

For Eq. (114), $\theta$ becomes Eq. (115) in radiant.

$$
\theta=-\pi \tan ^{-1}(2)-\tan ^{-1}\left(\frac{2 \cos \left(\pi \tan ^{-1}(2)\right) \sin \left(\pi \tan ^{-1}(2)\right)}{2 \cos \left(\pi \tan ^{-1}(2)\right)+\sin \left(\pi \tan ^{-1}(2)\right)}\right)
$$

For these values, $\beta=\alpha+\theta$ becomes Eq. (116) in radiant.

$$
\beta=-\tan ^{-1}\left(\frac{2 \cos \left(\pi \tan ^{-1}(2)\right) \sin \left(\pi \tan ^{-1}(2)\right)}{2 \cos \left(\pi \tan ^{-1}(2)\right)+\sin \left(\pi \tan ^{-1}(2)\right)}\right)
$$

As parametric equation of motion, it becomes Eq. (117),

$$
\begin{aligned}
\mathrm{x}(\mathrm{t})= & \cos (\alpha) \cos (\beta), \\
\mathrm{y}(\mathrm{t})= & \sin (\alpha) \cos (\beta), \\
& \mathrm{z}(\mathrm{t})=\sin (\beta) .
\end{aligned}
$$

where $\frac{\mathrm{dx}}{\mathrm{ds}_{2}}=\cos (\alpha), \frac{\mathrm{dy}}{\mathrm{ds}_{2}}=\sin (\alpha), \frac{\mathrm{dz}}{\mathrm{ds}}=\sin (\beta), \frac{\mathrm{ds}_{2}}{\mathrm{ds}}=$ $\cos (\beta)$ and ds $=1$ over Eq. (127).

Over the defined elements, presentation turns into Fig. 21.

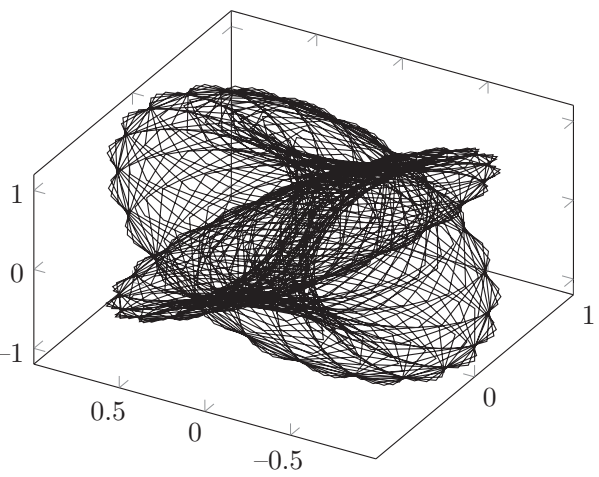

Fig. 21: Limited presentation of mass emergence by the parametric function.

It is a limited presentation, and actually it will be incredibly denser. Its curve length will be millions of unit. I did not use more sample to be more clear of it.

Fig. 21 is actually an attractor. It can only be valid in this shape at the beginning of time since the distance taken was all possible ways of the universe in the smallest time. As you can see over the above stated derivative components, $\mathrm{dx}$, $\mathrm{dy}, \mathrm{dz}$ are the smallest 3D derivative components of an unknown function whatever the function is; so as matter basically works over the same principle, namely if that components were for speed, distance, force or time by changing functions, the rule would be the same even component magnitudes change; so according to these component magnitude change, the attractor naturally changes for selected different intervals; but at the end, any motion closes to the rule of attractor.

Warning 
All right; but an angle cannot be uncertain; because uncertain angle creates time and thus motion, mass and area beyond abstract mathematics as the end of the numbers after the comma never will come. This already means that there is a motion. I think according to the emerging frequency, there is a sliding for each round according to a reference threshold time. Even if it can be assumed that there did not emerge a motion for this round because of the smallest time, also it can be assumed that there is a motion. It cannot be ignored. During this sliding, the assumed fixed angle's number after the comma emerges I think; so $\mathrm{n}$ will be very close number to 0 even if it is an uncertain number. Frequency will complete the gap to 0; but first, we need the same frequency for to make $n$ 0 . After that we need the same frequency to turn it without disrupting the angle; so wee need $\mathrm{f}^{2}$ frequency to create matter. For this small angle and so time, matter will be counted as at two different places at the same time or will be counted as it did not moved as there will no time difference between each round.

Also being $\mathrm{n}=\mathrm{t} \in \mathbb{Z}$ and the angles are in radiant, the parametric function can be determined over Eq. (109a), Eq. (109b) and Eq. (109c) where ds $=1$. There are always the same angles; but even so, t can be put instead of $\mathrm{n}$ where $\mathrm{t}$ is only integer; because even if $\alpha$ is not self inverse function, the inverse function of $\alpha$ can only change the appearance axis; so it does not differ that inverse function is used or not. We can analysis the shape or lengths over the main function as well. As also it can be seen, difference frequency creates different feature matter; so over $\mathrm{t}=\alpha=$ $(4 \mathrm{t}+1)\left(2 \pi^{3} \mathrm{t}^{2}+2 \pi^{2} \mathrm{t} \tan ^{-1}(2)\right)$, it becomes Eq. (118).

$$
\alpha=\frac{1}{4}\left(\frac{\mathrm{t}}{2 \pi^{3} \mathrm{t}^{2}+2 \pi^{2} \mathrm{t} \tan ^{-1}(2)}-1\right)
$$

Here, upper limit of $t$ must be very big number.

\subsubsection{Another way of presentation of mass emergence}

If you assume, that there is only 2D, then it becomes $\mathrm{R}=\sqrt{\mathrm{x}^{2}+\mathrm{y}^{2}}$. If you add one dimension more, also it must be equal to $R=\sqrt{x^{2}+z^{2}}$ as one of the axis always appears with the third one, where $R$ is the resultant of $3 \mathrm{D}$. As the result, if there is a circular motion or a motion closes a curve, motion only can emerge by this way as there is no alternative, and a space wave is always perpendicular to emerging mass as resultant mass or velocity always has two perpendicular component according to a reference axis. It means that force and velocity are perpendicular to each other; because actually force never changes. Force is applied to a part of absolute space, and then space gets shape by emerging frequency, according to work done. There is only 1 and the same work for the entire universe. If force increases, it will be fixed at last, and then frequency will increase. Moving thing is mass always; but as force and mass are together, you can assume that force moved.

As there is a single work for the entire universe, and as the work is done by the same force and fixed speed, either mass must decrease and increase by the parameters as the angle of the force application changes or displacement time must change by a space tension; because for a fixed speed, also acceleration is fixed. Acceleration and speed can be fixed as a result of the movement has repeat but to be absolute.

As it was said, if there is a $3 \mathrm{D}$ motion, a point mass or force turns around a 2D circle, and at the same time turns around a sphere for the same speed; because if the resultant of 3D is fixed for a circular motion, then if you assume that there is only $2 D$, then it becomes $R=\sqrt{x^{2}+y^{2}}$. If you add one dimension more, also it must be equal to

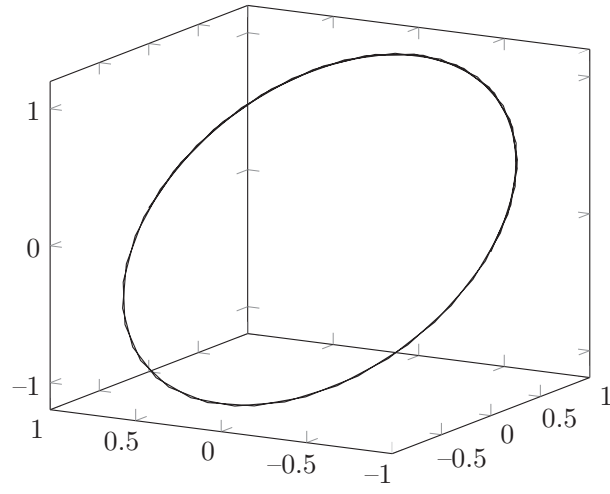

Fig. 22: Direction change graphic of the force applied, by the parametric curve of [ $\sin t, \cos t, \sin t, t, 0,2 \pi]$. Magnitude of the force applied is the same at any point of the elips; so as force is applied by the same intervals at a fixed speed, the changing thing is displacement time around circumference of the elips.

$\mathrm{R}=\sqrt{\mathrm{x}^{2}+\mathrm{z}^{2}}$ as one of the axises always appears with the third one, where $\mathrm{R}$ is the resultant of 3D. Already it can be seen over $\sqrt{\mathrm{F}^{2} \sin ^{2}(\alpha)+\mathrm{F}^{2} \cos ^{2}(\alpha)+\mathrm{F}^{2} \sin ^{2}(\mathrm{u})}=\mathrm{F}$. If $\mathrm{u}$ was a different angle like $\theta$, the equation would be $\sin ^{2}(\alpha)+\cos ^{2}(\alpha)+\sin ^{2}(\theta)=1$, and $\theta$ directly becomes $\alpha$ for a solution; and so the angles which emerge during 3D circular motion in $\mathrm{x}, \mathrm{y}$ plane way or in $\mathrm{y}, \mathrm{z}$ plane way at the same time where $\alpha=\mathrm{n} \pi$ and $\mathrm{n} \in \mathbb{Z}$, are always the same as it was said before the above; but the problem is it that, it never closes a curve. Even if it is not such that, it can be counted that a basic mass particle visits any point of space to create at an incredible frequency without turning back. It seems that there is a flow, and emergence area and its bodies move through infinite space.

There is no repeat which creates a closed curve. Any matter does a circular-like motion by having different places over time. As a motionless mass is not able to exist, also it is not able to move in the same place.

\section{Inference}

Even so, it may be counted as it closes a curve because of preservation of energy which is result of one-piece creation, just for calculations by some transformations.

Nobody said, that you can draw a closed curve. This does not mean eternal is limited, that is only an expression of eternal one. You can only increase the number of the side of a polygon. Namely, you can only use limits to explain or work about eternal. Still, God can forget a move easily since he can realize a collision in infinite precision; but since he always creates according to a rule and as already everything in a state of cause and effect relationship, he can call it back by calculating over the main function, by induction and deduction methods, and thus by creating from nothingness. Extremely interesting and eternal is able to create infinite things.

\section{Warning}

There are 3 possible 3D resultant equations as there is no alternative for uncertain motions. One of them becomes Eq. (119) over $\sqrt{\mathrm{F}^{2} \sin ^{2}(\alpha)+\mathrm{F}^{2} \cos ^{2}(\alpha)+\mathrm{F}^{2} \sin ^{2}(\alpha)}=\mathrm{F}$ 
equation.

$$
1=2 \sin ^{2}(\alpha)+\cos ^{2}(\alpha)
$$

There is a single root as following,

$$
\alpha=\mathrm{n} \pi
$$

where $n \in \mathbb{Z}$.

The other one becomes Eq. (120),

$$
1=\sin ^{2}(\alpha)+\cos ^{2}(\alpha)+\tan ^{2}(\alpha)
$$

where $\mathrm{dz}=\mathrm{F} \tan (\alpha)=\mathrm{F} \sin \alpha$. The roots are as following,

$$
\begin{gathered}
\alpha=\mathrm{n} \pi \\
\alpha=2 \mathrm{n} \pi+\pi
\end{gathered}
$$

where $n \in \mathbb{Z}$

Final one becomes Eq. (121).

$$
1=2 \cos ^{2}(\alpha)+\sin ^{2}(\alpha)
$$

The roots are as following,

$$
\begin{array}{r}
\alpha=\frac{1}{2}(2 \pi \mathrm{n}+\pi) \\
\alpha=\frac{1}{2}(2 \pi \mathrm{n}-\pi)
\end{array}
$$

where $n \in \mathbb{Z}$

Now that, if the roots are equalized to each other, the $n$ values which render possible to emerge of a motion appear as either -1 or 0 ; namely $\mathrm{n}=-1$ over $\mathrm{n} \pi=2 \mathrm{n} \pi+\pi$ or $2 \mathrm{n} \pi+\pi=\frac{1}{2}(2 \pi \mathrm{n}-\pi)$, and $\mathrm{n}=0$ over $2 \mathrm{n} \pi+\pi=\frac{1}{2}(2 \pi \mathrm{n}+\pi)$ for all possible defined combination, where $n \in \mathbb{Z}$; so the angle is only able to become as following.

$$
\begin{aligned}
& \alpha= \pm \frac{\pi}{2} \\
& \alpha= \pm \pi
\end{aligned}
$$

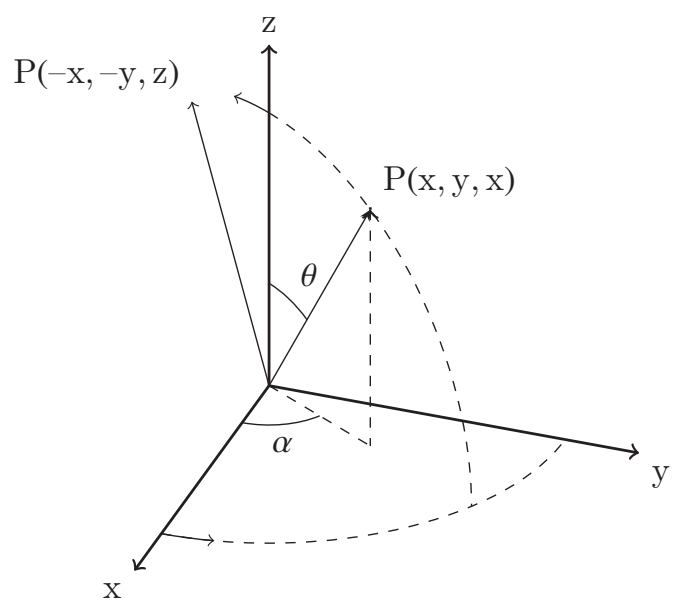

Fig. 23: The resultants

It means, that actually the 3D resultant is not at the same area with $\mathrm{dx}$ and $\mathrm{dy}$. For an assumed $\mathrm{P}(\mathrm{x}, \mathrm{y}, \mathrm{z})$ point of the resultant, it actually appears at $\mathrm{P}(-\mathrm{x},-\mathrm{y}, \mathrm{z})$ point for $+\mathrm{dx}$ and + dy. This is very interesting.

As all emergence work is together and emerges one by one, the emerging mass's own space has to have the same speed with the mass between $[0,1]$ second interval as an assumed and detected constant speed because of limited creation. The speed always must be constant because of the infinite derivatives which are stated the above since only deterministic image's frequency will change, even it decrease in time as each decrease interval is going to be the same again; so, assume that the smallest point of the space has $m_{\mathrm{S}}$ mass and $v$ fixed velocity for emerging $m$ mass which has the same $\mathrm{v}$ velocity.

If there was no time difference between space collisions or frictions, the resultant force on the mass would be zero, and mass would go at infinite speed or would not move for any magnitude force which creates speed of the mass, because of emerging zero resistance of the space. Motion always emerges in one direction as a resultant; because you can assume that there are infinite numbers of forces which cause motion in different directions; so as a resultant, motion only emerges in one direction since there cannot be an absolute zero resultant because of emerging infinite time differences; so as a resultant, whatever the opposite space resistance direction, motion always emerges by an angle in a direction which can be determined according to a reference that can be change relatively, and it turns into for example Fig. 24.

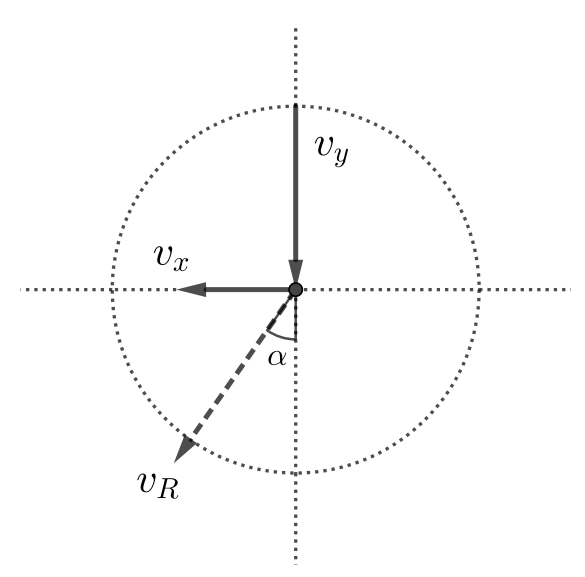

Fig. 24: The resultant movement

If this is the condition, the equation of the motion emerges as Eq. (124) or Eq. (125) for the same speeds,

$$
\begin{gathered}
\sqrt{\left(\mathrm{m}_{0} \mathrm{v}\right)^{2}+\left(\mathrm{m}_{\mathrm{s}} \mathrm{v}^{2}\right)}=\mathrm{mv} \\
\mathrm{m}=\frac{\mathrm{m}_{0}}{\sin (\alpha)}
\end{gathered}
$$

and it means, an uncertain matter always does a circular motion by this equation of a circle, and a space wave is always perpendicular to emerging mass as resultant mass or velocity has two perpendicular $\mathrm{x}, \mathrm{y}$ resultants always according to a reference axis. It means that force and velocity are perpendicular to each other; because actually the equation should be $\sqrt{\mathrm{v}^{2}+\mathrm{v}^{2}}=\mathrm{v}$; but it is insoluble; because for the limited and fixed speed which has to emerge for uncertain matter as there is no alternative even if the speed decreases in time as each time period will be assumed as fixed and average again, velocity turns into latency and so into mass by friction or by a small collision; so if a velocity emerges, it always perpendicular to force or emerging mass at the same time.

To find out how the angle changes even for the light which moves at light speed, you must use preservation of energy since all works can be done as much as energy existence and subject to the same rule; so over Eq. (65), Eq. (125) turns into 
Eq. (126) for $1-\frac{\mathrm{v}^{2}}{\mathrm{c}^{2}}=\sin (\alpha)$ where $1-\frac{\mathrm{v}^{2}}{\mathrm{c}^{2}}=\mathrm{m}_{0} / \mathrm{m}$.

$$
\alpha=\sin ^{-1}\left(1-\frac{\mathrm{v}^{2}}{\mathrm{c}^{2}}\right)
$$

Here $\mathrm{v}$ always takes values in $[0, \mathrm{c}]$ interval during 1 second for the average velocity of c. As it can be seen over Eq. (126), constant speed is not possible. When you detected a c speed magnitude in the equation, $\mathrm{v}$ from the same equation could not be $\mathrm{c}$ that actually it is c since already this does an emergence motion at the same space; but even before when you determined any $\mathrm{c}$ value, it changes that it always changes in any small time interval of any small time interval; so actually Eq. (126) does not become unsolvable even for $\mathrm{v}=\mathrm{c}$ since single $\mathrm{c}$ value always changes as it is irrational. $\mathrm{c}_{1}$ and $\mathrm{c}_{2}$ emerge as other variations of $c$ and you can use one of them instead of $\mathrm{v}$ in Eq. (126). As the result, $1-\left(\mathrm{v}^{2} / \mathrm{c}^{2}\right)$ could be both (+) and (-) as also could be very close to 1 from (+) and (-) directions by $0<\mathrm{v}^{2} / \mathrm{c}^{2}<1$ and $1<\mathrm{v}^{2} / \mathrm{c}^{2}<2$; so you can assume, that $1-\left(\mathrm{v}^{2} / \mathrm{c}^{2}\right)$ is nearly equal to 0 from both $(+)$ and $(-)$ direction and the angle becomes near zero from $(+)$ or (-) direction; but as there is no other motion, being the second speed $\mathrm{v}$ is nearly zero, the angle becomes nearly 90 . These two are also options; but different variations of c can only be used for formation wave namely each wave packet which creates particles. The other one is valid for outer space motion which is a result of many wave packets as particles.

If you add one extra dimension more, it stars to draw an helix according to the parametric equation of $\mathrm{x}=$ rcost, $\mathrm{y}=\mathrm{rsin} t, \mathrm{z}=\mathrm{ct}$ for $\mathrm{t} \in[0,2 \pi)$, where $\mathrm{r}$ is the radius of the helix and $2 \pi \mathrm{c}$ is a constant giving the vertical separation of the helix's loops. Drawing helix is an obligation as there is no alternative, since $m_{S}$ is not absolute at the closest distance to $\mathrm{m}$. The angle always changes. Components which emerge for $\mathrm{x}, \mathrm{y}, \mathrm{z}$ axis are perpendicular again, and total resultant determines the way. During the process, space turns into mass, and mass turns into space as waves over the infinite. Already, for a limited universe velocity can be counted as fixed, and so for a fixed speed different mass magnitudes are only possible with a circular motion; because different masses means different displacements and so different velocities. If radius increases, then for the same velocity, mass decreases as detected mass in an unit of volume is going to decrease.

\subsubsection{Space tension requirement}

Additionally, over ds as required by conservation of mass; namely, the relation between $\alpha$ and ds becomes Eq. (127) over $\mathrm{ds}=\mathrm{dx}+\mathrm{dy}+\mathrm{dz}$ when $\mathrm{dx}$, dy and dz from Eq. (109a), Eq. (109b) and Eq. (109c) are used on it,

$$
\mathrm{ds}=1
$$

where $\mathrm{m}=1 / \mathrm{v}$ over it. Over Eq. (127), it becomes Eq. (128) when $\mathrm{ds}=\mathrm{dx}+\mathrm{dy}+\mathrm{dz}$ is used on Eq. (107) instead of 1.

$$
-2 d x\left(\frac{1}{d y}+\frac{1}{d z}\right)=d x+d y+d z
$$

If it is edited, it becomes Eq. (128a),

$$
-2 d x \cdot d z-2 d x \cdot d y=d x \cdot d y \cdot d z+d y^{2} \cdot d z+d y \cdot d z^{2}
$$

after that becomes Eq. (128b),

$$
d y\left(2 d x+d x \cdot d z+d y \cdot d z+d z^{2}\right)=-2 d x \cdot d z
$$

after that becomes Eq. (128c),

$$
\frac{1}{\mathrm{dy}}=-\frac{1}{\mathrm{dz}}-\frac{1}{2}-\frac{\mathrm{dy}}{2 \mathrm{dx}}-\frac{\mathrm{dz}}{2 \mathrm{dx}}
$$

If $\frac{1}{\mathrm{dz}}$ is added to both of the sides, it becomes Eq. (128d),

$$
-\frac{1}{2 \mathrm{dx}}=-\frac{1}{2}-\frac{\mathrm{dy}}{2 \mathrm{dx}}-\frac{\mathrm{dz}}{2 \mathrm{dx}}
$$

where $\frac{1}{d y}+\frac{1}{d z}=-\frac{1}{2 d x}$ over Eq. (107). If it is edited, it becomes Eq. (128e).

$$
\mathrm{ds}=\mathrm{dx}+\mathrm{dy}+\mathrm{dz}
$$

It means, that verifying is successful, and momentum is always conserved; but if a mass moves on observational outer space, even if it is going to do this to protect its formation speed, its mass and total energy are going to increase even if the formation speed is still the same and fixed at that time, as also it was already proven in the physical medium section at the beginning; so the multiplication of mass and speed will not be the same for the fixed speed and increased mass; therefore as total momentum of the universe is always fixed, the universe is only able to allow to change in mass by space tension. Space can be bended.

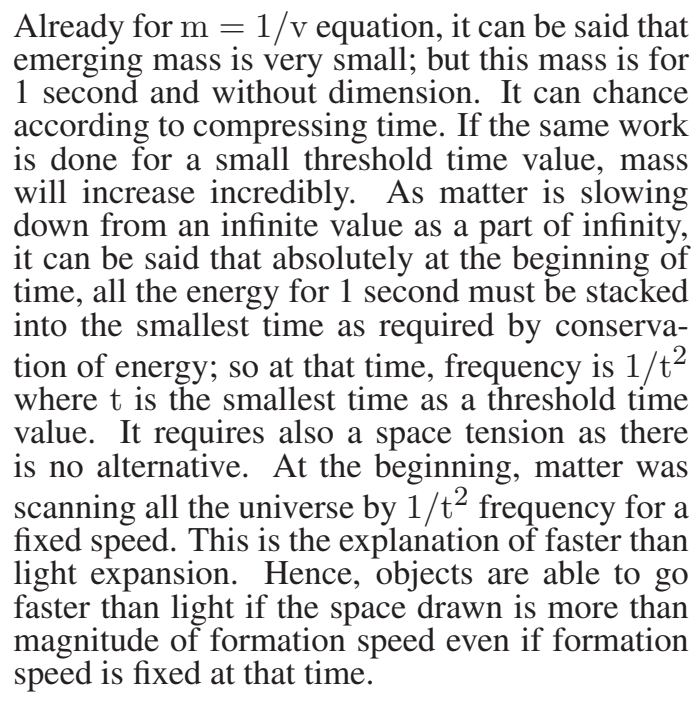

\section{Warning}

An assumed mass particle which has $m_{t}$ mass as threshold value forms $\mathrm{m}$ at a frequency; so over $\mathrm{m}=1 / \mathrm{v}$, it becomes $\mathrm{m}_{\mathrm{t}} \frac{\mathrm{v}}{\mathrm{x}}=\mathrm{m}$ where $\mathrm{x}$ is distance taken and $\mathrm{v}$ is formation speed. If both of the sides are multiplied by m, it becomes Eq. (129),

$$
\mathrm{x}=\mathrm{m}_{\mathrm{t}} \mathrm{v}^{2}
$$

where $\mathrm{m}_{\mathrm{t}} \frac{\mathrm{mv}}{\mathrm{x}}=\mathrm{m}^{2}, \mathrm{~m}_{\mathrm{t}} \mathrm{v}^{2}=\mathrm{E}$ is total energy of $\mathrm{m}_{\mathrm{t}}$ for 1 second, $\mathrm{mv}=1$ and $\mathrm{m}^{2}=1 / \mathrm{v}^{2}$. This ratio and equation are always fixed; but if it is not a perfect circle, only the values change. As required by conservation of energy, at the beginning of time, all the energy which will emerge after the smallest time during 1 second must exist at the smallest time potentially; so square of the frequency is only used for the beginning of time, and it turns into Eq. (130).

$$
\mathrm{x}=\mathrm{v} \sqrt{\mathrm{m}_{\mathrm{t}} \mathrm{V}}
$$

If the equation is $\mathrm{mv}=1$, then it means work done equa- 
tion turns into Eq. (131),

$$
\mathbf{F}=\frac{\mathrm{v}}{\mathrm{x}}
$$

where $\mathrm{Fx}=\mathrm{mv}^{2}$ and $\mathrm{mv}=1$. As it can be seen, force is equal to frequency. It means force is application repeat. If this is the condition, it is interesting and actually is very compatible being in accordance with conservation of energy, that even if mass of a particle is interpretable as $\mathrm{m}=\mathrm{m}_{\mathrm{t}} \mathrm{f}$ where $\mathrm{m}$ is particle mass, $\mathrm{m}_{\mathrm{t}}$ the basic mass and $\mathrm{f}$ is the frequency of the basic mass, this cannot emerge in this way. The basic mass is not free such that. For the same assumed basic formation mass particle, the equation becomes Eq. (132),

$$
\mathrm{a}=\mathrm{v}
$$

where $\mathrm{m}_{\mathrm{t}} \mathrm{v}^{2}=\mathrm{m}_{\mathrm{t}}$ ax and $\mathrm{x}=\mathrm{vt}$ for $\mathrm{t}=1$ second; but for the particles which the basic mass particle forms them, it turns into Eq. (133),

$$
\mathrm{a}=\frac{\mathrm{mv}}{\mathrm{m}_{\mathrm{t}}}
$$

where $\mathrm{m}$ is the mass formed by the assumed basic mass particle, $\mathrm{mv}^{2}=\mathrm{m}_{\mathrm{t}}$ ax and $\mathrm{x}=\mathrm{vt}$ for $\mathrm{t}=1$ second. It means that the basic mass gains more acceleration while forming particle. It does not repeat the same motion more in a limited area by the same acceleration to create a mass by creating more mass density in an unit of volume for changing radius at fixed speed. In the exact opposite, the basic mass which actually is named as photon is formed by a bigger acceleration, and is seen as particle. For these information, it can be said, that Planck time is not enough. There must be other smaller times which are in a big range due to mass scatter. Until it becomes $t_{c}<t_{e}$ for the same mass, where $t_{c}$ is creation time and $t_{e}$ is evaporation time, any time and any magnitude mass can be created, and they can behave like any particle.

Hence matter is always together with a space tension for any size motion, and speed can be independent of distance as it is relative. It means that matter is already in a jumping status. For a fixed speed, more distance can be taken by space tension by more energy or actually by more repeat as required by $m v=1$ and to be frequency of force. This is only possible by an absolute space. When absolute space is be deformed, energy and frequency emerge until to be fixed. To produce more energy, just you need to deform more space by a bigger volume; so even if the emerging energy is dependent of the force applied application speed, also is depended on how much space is used in volume. The same energy can be produced since work done is equal to kinetic energy by fast force application for a smaller space part or more space using instead of it by a smaller speed. The distance taken during these two different works can be the same even if the speed is different.

Already as required by $\mathrm{m}=\frac{1}{\mathrm{v}}$, if speed increases then mass decreases. This seems as paradox but it is not; because more speed is only possible in the smaller periods of time because of deceleration, and also the density of emerging universe will decrease for higher speeds because of bigger centrifugal force thus volume that it causes smaller mass magnitude for the same radius; so instead of using high speed, more space from absolute space can be used to create bigger universe.

I have not worked yet about its adaptation; but since is $\mathrm{P}=1$ where $\mathrm{P}$ is momentum, is also can be used for probability calculations since sum of all probabilities is equal to 1 .

Warning

\subsubsection{Existence probability of uncertain bodies}

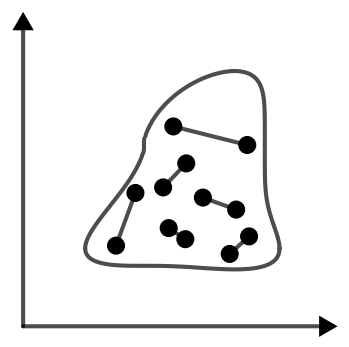

Fig. 25: Conservative area with a random closed curve

There is a random shaped closed curve on Fig. 25. Even if $2 \mathrm{D}$ is not possible, we are going to work on $3 \mathrm{D}$ over it.

Now think that some changing magnitude forces are applied to create mass in this area between two point. Any small point of are has emergence priority; thus they can never intersect even for increasing points; so if we turn the Fig. 25 into Fig. 26, it is also acceptable.

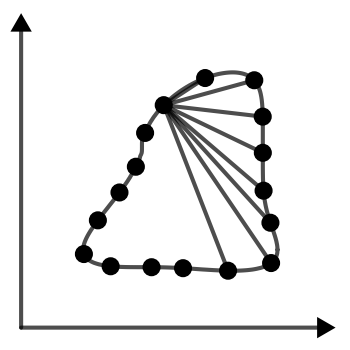

Fig. 26: Creating mass

On Fig. 26, assume that the force is applied from a point to the other all point on the circumference being the distance between the points are the same. For this, as there is emergence priority and thus time difference for each small point of area; being $\mathrm{n}$ is total point number on the circumference, it can be $\mathrm{n} \Delta \mathrm{l}_{1}$ as also can be $\mathrm{n}^{2} \Delta \mathrm{l}_{2}$ where $\Delta \mathrm{l}$ is average length of the used to create an area; thus it becomes $\Delta \mathrm{l}_{1}=\mathrm{n} \Delta \mathrm{l}_{2}$ over $\mathrm{n}^{2} \Delta \mathrm{l}_{2}=\mathrm{n} \Delta \mathrm{l}_{1}$. It means, if you draw all lines only from one point to the other all point, you need more points on the circumference to scan or create and thus to converge the area. If you draw lines from each point to the other all points, then you do not need more points on the circumference, and both of them are acceptable since there is not going to be intersection point of lines as stated the above. In the same manner, the equation also becomes Eq. (134),

$$
\mathrm{nE}=\mathrm{A}
$$

where $\mathrm{E}=$ Fr over average length on $\lim _{\mathrm{r}_{0} \rightarrow \mathrm{r}}\left(\mathrm{F}_{1} \mathrm{r}_{0}+\mathrm{F}_{2} \mathrm{r}_{0}+\right.$ $\ldots+\mathrm{F}_{\mathrm{n}} \mathrm{r}_{0}$ ) is total energy and $\mathrm{A}$ is total area. Here $\mathrm{E}$ and $\dddot{A}$ does not converge over $\lim _{n \rightarrow \infty}$; instead of it $\mathrm{A}$ is always fixed; so if you write $\lim _{\mathrm{n} \rightarrow \infty} \mathrm{E}=\mathrm{A}$, it means zero energy which the area $\mathrm{A}$ on the horizon holds.

Assume that you have a square has $\mathrm{r}$ side in accordance with abstract math. If you draw the closest parallel $r$ lines to this side, you obtain the square and its $\mathrm{r}^{2}$ area; but also if you draw the 
lines as perpendicular, you obtain $2 \mathrm{r}^{2}$ area. If you constantly repeat it, being $\mathrm{f}$ is repeat number, it has $\mathrm{fr}^{2}$ area; but in accordance with the time difference, it cannot exist such since there cannot be intersection point; so since there are infinite time differences and thus space allows this, during the process, the energy which the area holds decreases as it can be seen over Eq. (134) being the area is the same. This creates a density concept such as energy density or mass density which the area holds. Area does not specify energy, distance or mass. Assume that there are infinite kind of free space in absolute space; then area is the part which you closed on horizon as imaginary to calculate the physical values of the space which is in the borderlines of the area you closed. Space could be created in any way by changing physical values as independent on the area you closed. As a result, $\mathrm{E}$ is dependent on $\mathrm{n}$ inversely proportional being $\mathrm{E}$ is $\mathrm{E}(\mathrm{n})$.

\section{Warning}

Any curve which is assumed as closed can also be defined in the kind of a circle for $\mathrm{r}=\sqrt{\mathrm{A} / \pi}$ radius over $\mathrm{A}=\pi \mathrm{r}^{2}$. Already any closed curve must be circle at the end if there is motion. Namely, if you take center of gravity for closed curves as a reference, it becomes $\left|\mathrm{F}_{\mathrm{p}_{+} \mathrm{x}} \mathrm{r}_{+\mathrm{x}}-\mathrm{F}_{\mathrm{p}_{-} \mathrm{x}} \mathrm{r}_{-\mathrm{x}}\right|>0$ in absolute value where $\mathrm{r}_{+\mathrm{x}} \neq \mathrm{r}_{-\mathrm{x}}$ and $\mathrm{F}_{\mathrm{p}}$ is partial force applied on a line in the area; but it becomes $\left|\mathrm{F}_{\mathrm{p}_{+} \mathrm{x}} \mathrm{r}_{+\mathrm{x}}-\mathrm{F}_{\mathrm{p}_{+} \mathrm{x}}\right| \approx$ 0 in absolute value where $r_{+x}=r_{-x}$ since the path for any force-applied is the same in circle as radius, and $\mathrm{F}_{\mathrm{p}}$ is partial force applied on a line in the area. It is approximate 0 since there is actually time differences and thus actually for the same time also force magnitude or distance taken cannot be the same in different direction. As a result, it becomes $\left|\mathrm{F}_{\mathrm{p}_{+} \mathrm{x}} \mathrm{r}_{+\mathrm{x}}-\mathrm{F}_{\mathrm{p}_{-} \mathrm{x}} \mathrm{r}_{-\mathrm{x}}\right|>\left|\mathrm{F}_{\mathrm{p}_{+} \mathrm{x}} \mathrm{r}_{+\mathrm{x}}-\mathrm{F}_{\mathrm{p}_{+} \mathrm{x}}\right|$ in absolute value. The difference in force in closed curves which are not circle also creates motion in accordance with the definition of force since it will be $\left(\mathrm{F}_{1}-\mathrm{F}_{2}\right) / \mathrm{t}=\mathrm{v}_{\mathrm{F}}$ where $\mathrm{t}$ is time of change in force and $v_{F}$ is its velocity or will be $m_{1} a_{1}-m_{2} a_{2}=m a$; so they are stressed and they want to take themselves to lower energy state, and at the end, to minimum energy state for its any point since denser points has more energy; but as it was said, this creates motion.

Hence the other variation of $\Delta \mathrm{l}_{1}=\mathrm{n} \Delta \mathrm{l}_{2}$ and thus of Eq. (134) as $\mathrm{nA}=\mathrm{E}$ is impossible; because matter moves from the denser space to lower density naturally. Forcing it to denser space is also moving to lower density since still matter emerges at that time by moving to lower density in inner space. Namely, natural characteristic of matter is not dependent on $\mathrm{nA}=\mathrm{E}$ since for the fixed $\mathrm{A}$, it is going to be $\lim _{n \rightarrow \infty} \mathrm{E}=\infty$. It cannot allow emergence of motion.

Over Fig. 27, it becomes Eq. (135) where $\alpha=360 / \mathrm{n}$ and $\mathrm{AB}=\mathrm{d}$ and $\mathrm{E}_{2}=\mathrm{nFr} / \mathrm{n}$ assuming $\mathrm{n}$ goes to infinite.

$$
\mathrm{d}^{2}=2 \mathrm{E}_{2}^{2}-2 \mathrm{E}_{2}^{2} \cos (\alpha)
$$

Also it becomes Eq. (136) over nd $=2 \pi \sqrt{\mathrm{A} / \pi}$ for the circumference of the circle where $\pi \mathrm{r}^{2}=\mathrm{A}$ assuming $\mathrm{n}$ goes to infinite.

$$
\mathrm{d}^{2}=\frac{4 \pi \mathrm{A}}{\mathrm{n}^{2}}
$$

If Eq. (135) and Eq. (136) are equalized to each other, then we get Eq. (137),

$$
1-\cos (\alpha)=\frac{2}{\mathrm{r}^{2}}
$$

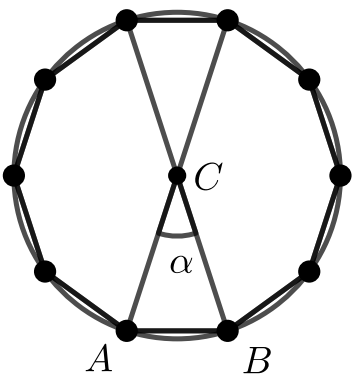

Fig. 27: Polygon transformation being $n$ is even number

where $\alpha=360 / \mathrm{n}$. Here assume that $\cos (\alpha)$ is the sum of all possibilities of possible existence of $\mathrm{F}$ and $\mathrm{r}$ relatively to each other since $\cos (\alpha)$ changes over $\alpha=360 \mathrm{~F} / \pi \mathrm{r}$ where $\mathrm{n}=\mathrm{A} / \mathrm{E}$. Namely all possible $\mathrm{F}$ and $\mathrm{r}$ values are in this area for $\int \cos (\alpha)$ dr. Also you can use $\int(1-\cos (\alpha)) d r$ as an option. For $\int \cos (\alpha)$ dr, we have Eq. (138),

$$
1=\frac{360 \mathrm{x}}{\pi} \mathrm{Si}\left(\frac{360 \mathrm{x}}{\pi \mathrm{y}}\right)+\mathrm{y} \cos \left(\frac{360 \mathrm{x}}{\pi \mathrm{y}}\right)
$$

where $\mathrm{x}=\mathrm{F}$ and $\mathrm{y}=\mathrm{r}$. Do not think the functions allow $\mathrm{n}=\infty$; because it creates infinite probabilities. In the exact opposite way, matter is uncertain as convergent. Namely $n$ has an end. Otherwise matter would not be created since $\mathrm{E}$ is going to become 0 ; so for the calculation $\pi$ is going to be a decimal number for required approximation.

There are some special conditions. First of all, force or distance is not continuous on $\mathrm{x}=\mathrm{F}$ and $\mathrm{y}=\mathrm{r}$ or $\mathrm{x}=\mathrm{r}$ and $\mathrm{y}=\mathrm{F}$ axis. Even if you take the values like $\mathrm{x}=\mathrm{F} / \mathrm{r}$ and $\mathrm{y}=\mathrm{r}$ or $\mathrm{x}=\mathrm{r}$ and $\mathrm{y}=\mathrm{F} / \mathrm{r}$, the rule must be the same. When one of them becomes 0 , the other one can exist but requires another dimension. It is not a paradox or calculation mistake since there is a 3D motion.

\section{Warning}

Already $\mathrm{F}=0$ is estimated result since we cannot know both of them at the same moment together, as it was proven above over derivative components.

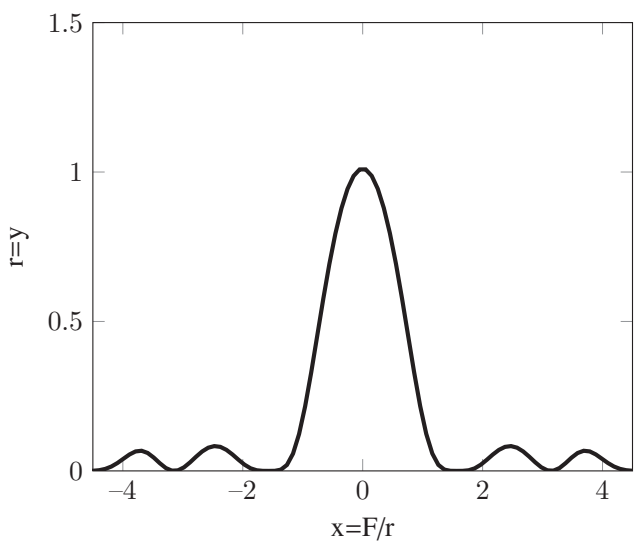

Fig. 28: Representation of change in existence of force and distance. 
For the second option, over $\int 1-\cos (\alpha) \mathrm{dr}=\int \frac{2 \mathrm{~F}}{\mathrm{r}^{2}} \mathrm{dr}$, it becomes Eq. (139),

$$
\mathrm{y}=\frac{\frac{180 \mathrm{xy}}{\pi} \operatorname{Si}\left(\frac{360 \mathrm{x}}{\pi \mathrm{y}}\right)}{1-\cos \left(\frac{360 \mathrm{xy}}{\pi \mathrm{y}}\right)}
$$

where $x=F / r$ and $r=y$. Since is $y=f(x, y)$, do not make a simplification for $y$ and accept it as $f(x, y)=1$; so it turns into Eq. (140).

$$
\mathrm{y}=\frac{\pi\left(-1+\cos \left(\frac{360 \mathrm{x}}{\pi}\right)\right.}{180 \mathrm{xSi}\left(\frac{360 \mathrm{x}}{\pi}\right)}
$$

For this equation, both force and distance become zero now and again it means, there is a circular motion and dimensional change. Already because of the time differences, there cannot be a middle point as also was proven at the beginning; so for any force-applied, a circular motion emerges.

\section{Area effect on optics}

As total energy is dependent on $\mathrm{nE}=\mathrm{nFr}=\mathrm{mc}^{2}$, it becomes Eq. (141).

$$
\lim _{n \rightarrow \infty} \frac{m c^{2}}{n r}=F=0
$$

Since force is sum of both circular displacement and moving to more ordered area at the same time together because of time differences, it actually means $\mathrm{F}_{\mathrm{G}}+\mathrm{F}_{\mathrm{C}}=0$ and thus means Eq. (142),

$$
\mathrm{F}_{\mathrm{G}}=-\mathrm{F}_{\mathrm{C}}
$$

or meansEq. (143) over $\lim _{\mathrm{n} \rightarrow \infty} \frac{\mathrm{mc}^{2}}{\mathrm{nr}}=\mathrm{F}_{\mathrm{C}}+\mathrm{F}_{\mathrm{G}}$ where $\mathrm{F}_{\mathrm{C}}=\mathrm{mc}^{2} / \mathrm{r}$.

$$
\lim _{n \rightarrow \infty} \frac{m c^{2}}{r}\left(\frac{1}{n}-1\right)=-\frac{m c^{2}}{r}=F_{G}
$$

It means, matter can move in two different type as distributed on area or as focused since when $n$ is infinite, it is like a linear motion; but actually n cannot be infinite, for focusing, you can only see the part of a higher dimension that actually is $3 \mathrm{D}$ in lower dimension that actually is $2 \mathrm{D}$ due to the changing values of in interval that it does not have to be for only creating, that is possible after creation for focal points of universe over partial forces or distances of total values. These also mean that the actual equation is Eq. (144),

$$
\mathrm{F}_{\mathrm{G}} \approx-\mathrm{F}_{\mathrm{C}}
$$

where $\mathrm{F}_{\mathrm{G}}>\mathrm{F}_{\mathrm{C}}$ or $\mathrm{F}_{\mathrm{C}}>\mathrm{F}_{\mathrm{G}}$ that one of them may always valid, or by changing intervals there is a constant transformation from one of them to the other one due to conditions.

Here actually 2D means both 1D and 3D. Actually it means still 3D; but depth and width are too close to a threshold value; so also you can assume that is $1 \mathrm{D}$ instead of $2 \mathrm{D}$ since it is going to be counted as without area or volume. Also it seems that it is related with the famous mass gap of Yang-Mills.

Warning
How we can describe the equality or the inequality as axial? It can only become Fig. 29, and it is insufficient even if the angles can change anywise.

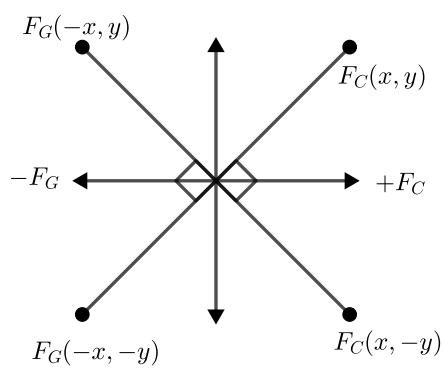

Fig. 29: Gravity and centrifugal force

It is insufficient; because on one axis, only one of them can be placed. It means Riemann surfaces are in the question, and complex analysis is required. The symmetry in the universe seems central symmetry.

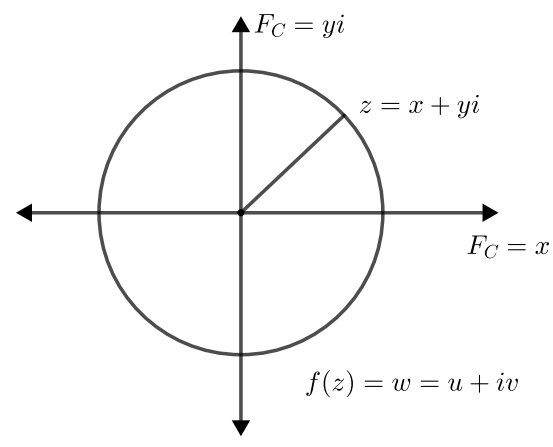

Fig. 30: Complex plane

Namely if I should show both focusing and complex placement together, it becomes Fig. 30. Here real and imaginary parts are intersected at the middle of the quarter circle. Somehow, it is like it, that neither imaginary part nor real part does not increase from 0 or does not decrease to 0 . Just the point slides slowly since is $\mathrm{F}_{\mathrm{G}} \approx-\mathrm{F}_{\mathrm{C}}$. Here is like an intersection point of motion between dimensions. Finally over it, it becomes Fig. 31.

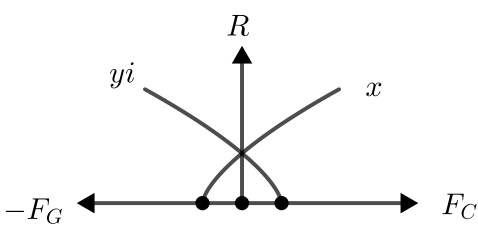

$$
w=f(z)
$$

Fig. 31: Complex plane for the image set 
As the focusing again, for example over Fig. 32, the focused matter appears more around black and white area instead of the other grey colors between black and white, according to your duplicating direction. This shape is only for example. It is not identical.

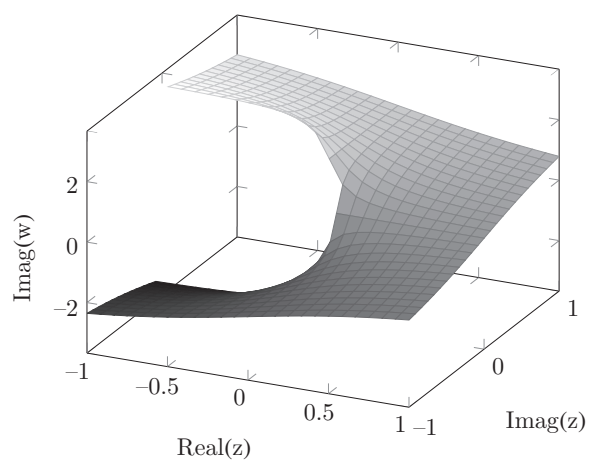

Fig. 32: Single branch of a random Riemann surface

\section{Natural relativistic mass}

In accordance with these findings, gravity is almost equal to centrifugal force if you do not include the time differences that as it was proven, if you include it, as long as distance increased, centrifugal force increases, and is the reason of dark matter misunderstanding. There is no dark matter. Linear attraction or momentum turns into angular momentum. This is not boosting; but is transformation. Orbital objects cannot fly off; but they get faster. Gravity does not work as Newtonian. Distance and gravitational force relation changes over distance. The attraction properties change for each point of free space, and have some limits. The attraction changes due to some values between $1 / \mathrm{r}$ and $1 / \mathrm{r}^{2}$

Since mass is gained by motion and motion is circular, centrifugal force as a result of circular displacement causes some special attraction properties in mass creation.

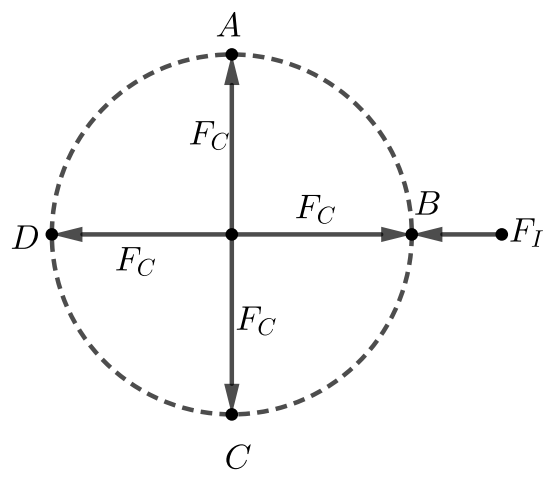

Fig. 33: Natural relativistic mass

Mass is a varying phenomenon, and as a result of this, also attraction is varying. It can seen some times like an external energy is working. Namely you can suppose that energy is not conservative since incredibly small energies can do work much more than their total energies. This is possible, and is dependent on some conditions.
Imagine that the 2D circle is a particle on Fig. 33. Naturally a single wave with a fixed mass and energy winds it, and the particle gains more mass if the frequency gets higher since detected mass of the basic wave in an unit of volume increases. Know that shape can be different, and actually it cannot be static since force is not continuous at every point of the circumference at the same time. It draws circular paths on space; but assume it is static. It is not important now.

Now if you apply a force to push it from B point, if the particle has enough frequency on the surface, naturally no object can pass from $B$ point since any point on the circumference will be like it is full of mass even is not; but if the frequency is low relatively, exactly required particles can pass inside of the particle without collision or friction with the surface wave. When $\mathrm{F}=\mathrm{ma}$ is handled, the mass here is the result of the condition. Namely some directions of the centrifugal force of the surface wave are in the same direction with the external force-applied in outer space over $\mathrm{F}=\mathrm{ma}$; but also some of them are in the opposite direction. Hence when you talk about mass, it can only sensed and detected in this way; then think that this particle is spinning around another object like in atoms. What happens if the direction of external force over $\mathrm{F}=$ ma becomes the same with the other object's direction of surface wave. For this condition, relative mass decreases. They get the smallest work value since resistance gets closer to zero for both attraction and repulsion. This depends on how fast they spin around each other, and how many mass they have. Atomic and sub-atomic particles may be inclined to spin in a state of resonance; thus also the emerging attraction or repulsion incredibly increases. This increase is such an increase that no oil can do this. Also resonance is not required. It can occur between some limits by changing values.

It seems that electrons can also lower the mass of nucleus by this way if it gets enough spinning speed and thus frequency, as also can increase. It also means that if you induce an electric charge on a material by AC current not DC, then if you catch the correct frequency, you can make matter worked by its own energy by a phenomenon like almost teleportation against gravity. You do not need so much energy. Even incomparably small energy can realize this. Timing and frequency change everything. It also means, for example earthquakes can be created without ELF waves or atmosphere can be used to send electricity over air molecules almost without resistance to the other side of the world by small energies. Even it can be focused somehow theoretically.

\section{Warning}

\subsubsection{Expansion of universe}

Handle $\mathrm{L}$ as distance taken which a gravitational $\mathrm{F}_{\mathrm{G}}$ force can pull back an accelerated mass and lower its speed in the smallest $\mathrm{t}$ time as threshold time value. For $\mathrm{F}_{\mathrm{G}} \mathrm{L}=\frac{\mathrm{m}_{\mathrm{t}} \mathrm{v}^{2}}{\mathrm{t}}$ where $\mathrm{v}$ is formation speed and mass is $\mathrm{m}=\frac{\mathrm{m}_{\mathrm{t}}}{\mathrm{t}}$ being $\mathrm{m}_{\mathrm{t}}$ is threshold mass value, it becomes Eq. (145).

$$
\mathrm{m}_{\mathrm{t}}=\frac{\mathrm{F}_{\mathrm{G}} \mathrm{t}^{2}}{\mathrm{v}}(\mathrm{Kg})
$$

The mass is $m=\frac{m_{t}}{t}$; because all energy that universe has for 1 second must exist for the smallest time in the beginning of time potentially. All the formed energy during this 1 second 
could be stacked into this small time. Over Eq. (145),

$$
\mathrm{t}=\sqrt{\frac{\mathrm{m}_{\mathrm{t}} \mathrm{v}}{\mathrm{F}_{\mathrm{G}}}}(\mathrm{s})
$$

The smallest length for this time becomes Eq. (147).

$$
\mathrm{L}=\sqrt{\frac{\mathrm{m}_{\mathrm{t}} \mathrm{v}^{3}}{\mathrm{~F}_{\mathrm{G}}}}(\mathrm{m})
$$

The mass stated the above as Eq. (145) is the mass which does not let light off in the smallest threshold time. This mass then has $\mathrm{m}=\frac{\mathrm{F}_{\mathrm{G}}}{\mathrm{V}}(\mathrm{Kgs})$ mass magnitude as it completes its motion for 1 second for the same radius. The force caused by the centrifugal acceleration emerging due to this mass becomes $\mathrm{F}_{\mathrm{C}}$ for the radius of $\mathrm{r}=\mathrm{L} / \mathrm{C}$ where $\mathrm{L}$ is Eq. (147) and $\mathrm{C}$ is a multiplier according to the circumference of the closed curve since the smallest distance is this over Cr. The distance taken is $\mathrm{x}=\mathrm{a}=\mathrm{c}^{2} / \mathrm{r}$ where $\mathrm{c}$ is the light speed for 1 second; so let the distance taken to be $\mathrm{a}_{\mathrm{C}}$ as centrifugal force acceleration and is also radius of the emerging universe without particle. A strong vacuum area emerges due to this force. Universe uses its own heat to work; thus also it loses heat during this work as well; thus space is always cold. As the light velocity made universe stable, it performs only the act of emergence, and the universe does not expand after the expansion for 1 second. As all actions can be performed as much as total energy of universe or used matter, based on the action performed in the direction of centrifugal acceleration, the mass emerging as a result of this action performed becomes Eq. (148),

$$
\mathrm{m}_{\mathrm{U}}=\frac{\mathrm{F}_{\mathrm{C}} \mathrm{a}_{\mathrm{C}}}{\mathrm{v}^{2}}(\mathrm{Kgs})
$$

where $\mathrm{v}$ is formation speed. The density of the universe also becomes Eq. (149),

$$
\mathrm{d}_{\mathrm{U}}=\frac{\mathrm{F}_{\mathrm{C}_{\mathrm{C}}}}{\mathrm{V}_{\mathrm{UV}^{2}}}\left(\mathrm{Kgs} / \mathrm{m}^{3}\right)
$$

where $\mathrm{V}_{\mathrm{U}}$ volume of the universe; but the universe can only possess this density when all of the particles of the universe evaporated. As matter emerges over absolute space by infinite time differences, if it draws extra space during emergence as emergence and outer space motion are accepted together, it can go faster than formation speed relatively even if the speed is still fixed at that time. This is caused by space tension. The universe was able to expand faster than light for 1 second at the beginning of time by this way. All possible distances were taken in the smallest time and in the smallest singularity point as a reference.

As it can be seen, the universe has a center and the thing which seems as expanding is caused by the photons which create particles, and also by evaporation as the universe emerges due to centrifugal force as radius will change by evaporation in the other name decrease or stop in vibration because of frictions with space since particles have no external energy. During the expansion, a potential difference can emerge along the space which are along the direction of centrifugal acceleration because of the initial movement, and masses move by photons' momentum because of emerging photon friction with space. Matters' own creation motion which is momentum in the other name causes outer space movement by friction. Also this is a part of gravity. Spooky action at a distance of Einstein [3] may also be caused by superposition of the universe which is because of $1 / t^{2}$ Hertz frequency at the beginning of time or after expansion $1 / \mathrm{t}$ Hertz for 1 second. As it does not differ for universe, that is it a particle, the universe can touch all of them almost at the same time from the furthest existent distance by a little time difference.

A space contraction may be seen at some points of space because of the mass scatter as the universe emerges by centrifugal force. These points are denser. If we look at the information, it can be said that the waves intertwined together and they formed the subatomic particles, and then hydrogen or maybe different unknown atoms which we cannot reach them because of the mass scatter by an ignored time difference; thus diffuse of particles is not so hard in the universe in 1 second. All of the work is together and is in 1 second. If many masses cross on the same point at the same time then space must be collapsed and then it must be swollen, and gravity will keep them together; thus new types of elements may be done by photon-photon collisions, and they don't have to be like in our scientific knowledge. Supercomputers will be saver to do it.

Additionally, the basic wave which creates particles or moves in space freely does not draw a perfect circle or sphere. Conservation of momentum and energy equations do not let it. Even so, if you assume, that is a perfect circle that actually will be a little difference, to understand the actual measurements closer manner, we can do some calculations which are not so perfect.

Namely, the distance taken every second in a particle or empty space must be $\mathrm{r}=\mathrm{c} / 2 \pi$ for $2 \pi \mathrm{r}=$ ct and $\mathrm{t}=1$ second. During the work, photon acts like a particle when $\mathrm{r}=\mathrm{c} / 2 \pi$ radius is counted as free circulation radius, and the photon has the same values like its formed ones for 1 second in each work. The area covered must be Eq. (150),

$$
\mathrm{A}=\mathrm{c}^{2} / 4 \pi\left(\mathrm{m}^{2}\right)
$$

based on $\pi \mathrm{r}^{2}$. For e $(\mathrm{Js})$, as it emerges within 1 second, energy density in this area is Eq. (151),

$$
\mathrm{d}_{\mathrm{e}}=4 \pi \mathrm{e} / \mathrm{c}^{2}\left(\mathrm{Js} / \mathrm{m}^{2}\right)
$$

and it is assumed, that e energy provides this equitation for $1 \mathrm{~m}^{2}$. The energy generated in 1 dimension along 1 meter for 1 second is $\sqrt{\mathrm{d}_{\mathrm{e}}}(\mathrm{Js})$; thus it will be Eq. (152),

$$
\mathrm{E}=4 \pi / \mathrm{c}^{4}(\mathrm{Js})
$$

based on $\mathrm{e}=\sqrt{\mathrm{d}_{\mathrm{e}}} / \mathrm{c}\left(\mathrm{Js}_{\mathrm{s}}\right)$ for each distance taken at the speed of light of $\mathrm{c}$, each second. This could be the real required Planck constant as closer. This is the total energy of the basic creation wave named as photon which has 1 Hertz frequency, and it occurs along 1 second in 1 dimension. The mass creates this energy is Eq. (153)

$$
\mathrm{m}_{\mathrm{ph}}=4 \pi / \mathrm{c}^{6}(\mathrm{Kgs})
$$

based on $\mathrm{e} / \mathrm{c}^{2}$ (Kgs), and this is the photon mass which can be shorten as $m_{\mathrm{ph}}$. This is the mass which can only be formed along 1 second; thus when it collides with an object, the total mass effect occurs at the end of 1 second; thus wave can only be a string. The force which causes this mass will be $\mathrm{f}=$ $\mathrm{ma}=\mathrm{mv}$ for 1 second; thus it must be

$$
\mathrm{F}=4 \pi / \mathrm{c}^{5}(\mathrm{Kgs})
$$

In the same manner, over perfect circular motion assumption, to calculate the over stated values of the universe by these, let us assume, that $\mathrm{x}$ is the distance which the gravitational $\mathrm{F}$ force can pull back an accelerated mass and lower the speed of the mass in a t time. For $\mathrm{m}^{2} \mathrm{Gx} / \mathrm{x}^{2}=\mathrm{mv}^{2}$, it 
will be $\mathrm{v}=\sqrt{\mathrm{mG} / \mathrm{x}}$. For $\mathrm{x}=\mathrm{vt}, \mathrm{c}$ is the speed of light and $t$ is the smallest time allows this work, then it will be $\mathrm{c}=\sqrt{\mathrm{mG} / \mathrm{ct}}$. Here if $\mathrm{c}, \mathrm{t}$ and thus $\mathrm{m}$ are constant as required by uncertainty, then also $\mathrm{G}$ must be constant. The total mass of the universe is going to be Eq. (155) for this small escape time of $t$.

$$
\mathrm{m}=\mathrm{tc}^{3} / \mathrm{G}(\mathrm{Kg})
$$

The frequency of this mass is going to be $1 / \mathrm{t}$ for this time. Formation is not only along 1 second for the beginning of time. After 1 second, the photon mass must form a black hole for 1 second according to its own rule that photon mass occurs for 1 second or again a space density must allow photon to move at the light speed. It is a necessity due to mass and energy conservation. All the energy which the universe possess must exist for 1 Planck time in the beginning of time potentially. All the formed energy during this 1 second could be stacked into this small time as it was said. The frequency is $1 / \mathrm{t}^{2}$ at the beginning. After 1 second, it decreases and becomes $1 / \mathrm{t}$.

For $\mathrm{m}_{\mathrm{ph}}=4 \pi / \mathrm{c}^{6}(\mathrm{Kgs})$ and $\mathrm{m}_{\mathrm{ph}}=\mathrm{t}^{2} \mathrm{c}^{3} / \mathrm{G}$, the Planck time is going to be Eq. (156).

$$
\mathrm{t}=\sqrt{4 \pi \mathrm{G} / \mathrm{c}^{9}}(\mathrm{~s})
$$

The Planck length for this time is going to be Eq. (157).

$$
\mathrm{L}=\sqrt{4 \pi \mathrm{G} / \mathrm{c}^{7}}(\mathrm{~m})
$$

The constants emerging with the required setting can be found. The mass stated the above as Eq. (155) is the mass which does not let light off in 1 Planck time. This mass then has $\mathrm{m}=\mathrm{c}^{3} / \mathrm{G}$ (Kgs) mass magnitude as it completes its motion for 1 second for the same radius. The force caused by the centrifugal acceleration emerging due to this mass is going to be Eq. (158) as the below

$$
\mathrm{F}=\sqrt{\pi \mathrm{c}^{17} / \mathrm{G}^{3}}(\mathrm{Kgs})
$$

being $\mathrm{r}=\mathrm{L} / 2 \pi(\mathrm{m})$ for 1 second. A strong vacuum area emerges due to this force. Universe uses its own heat to work; thus also it loses heat during this work as well; thus space is always cold. The acceleration emerging for this force is going to be Eq. (159),

$$
\mathrm{a}=\sqrt{\mathrm{c}^{11} / 4 \pi \mathrm{G}}\left(\mathrm{m} / \mathrm{s}^{2}\right)
$$

where $\mathrm{a}=\mathrm{c}^{2} / \mathrm{r}$. The path proceeded for the reference time of 1 second based on $\mathrm{x}=\mathrm{at}^{2}$ is going to be Eq. (160) as the below.

$$
\mathrm{x}=\sqrt{\mathrm{c}^{11} / 4 \pi \mathrm{G}}(\mathrm{m})
$$

This is the radius of the universe. As the light velocity made the universe stable for the higher values which are stable, it performs only the act of emergence, and the universe does not expand after the expansion for 1 second. As all actions can be performed as much as total energy of universe or used matter; based on the action performed in the direction of centrifugal acceleration, the mass emerging as a result of this action performed is going to be Eq. (161).

$$
\mathrm{m}=\mathrm{c}^{12} / 2 \mathrm{G}^{2}(\mathrm{Kgs})
$$

It can be said, that rather nonsense physical phenomena in the universe are possible for such a big mass and they can occur at any place each second according to the total number of the particles emerging as well. The density of the universe is going to be Eq. (162)

$$
\mathrm{d}=\sqrt{9 \pi / \mathrm{Gc}^{9}}\left(\mathrm{Kgs} / \mathrm{m}^{3}\right)
$$

; but the universe can only possess the density when all of the particles of the universe evaporated. The universe has a center and the scatter cannot be homogeneous, and is not going to be because of time differences. Irregularity dominates always.

In the same manner, over perfect circular motion assumption again, energy of a photon which has 1 Hertz frequency must be Eq. (163) as Max Planck derived it [6].

$$
\mathrm{E}=\mathrm{h} v\left(\mathrm{Js}_{\mathrm{s}}\right)
$$

Photon performs a single motion to form particle. It closes a curve and thus surface, and frequency of a photon on a particle surface determines the radius of particle; thus $v$ being the radius of a free particle which does not consists of other subatomic particles, $v$ being the photon's frequency of revolution around the particle, $m_{p}$ being the mass of the particle, $m_{p h}$ being the mass of the photon during 1 second, for $\mathrm{m}_{\mathrm{ph}} v=\mathrm{m}_{\mathrm{p}}$ and $\mathrm{c} / 2 \pi \mathrm{r}=\mathrm{m}_{\mathrm{p}} / \mathrm{m}_{\mathrm{ph}}$ over $\mathrm{m}_{\mathrm{ph}} v=\mathrm{m}_{\mathrm{p}}$ where $v=\mathrm{c} / 2 \pi \mathrm{r}$, free single particle radius must be Eq. (164).

$$
\mathrm{r}_{\mathrm{p}}=\frac{\mathrm{h}}{2 \pi \mathrm{m}_{\mathrm{p}} \mathrm{c}}(\mathrm{m})
$$

This radius can be vary based on the place it exists in space. With another formulation, $h$ being the Planck constant, as it will be $\mathrm{m}_{\mathrm{p}} \mathrm{c}^{2}=\mathrm{h} v$, it must be Eq. (165)

$$
\mathrm{m}_{\mathrm{p}}=\mathrm{h} v / \mathrm{c}^{2}(\mathrm{Kgs})
$$

These are hypothetical and indirect calculations for the available values as there is no alternative as well. The detected force of a particle as the result of its circular displacement due to a mass must be Eq. (166) over centrifugal force where $\mathrm{L}=2 \pi \mathrm{r}$ since the smallest distance is circumference of a circle, $\mathrm{r}=\frac{\mathrm{h}}{2 \pi \mathrm{m}_{\mathrm{ph}} \mathrm{c}}$ over Eq. (164), $\mathrm{m}_{\mathrm{ph}}$ is mass of photon at 1 $\mathrm{Hz}$, and $\mathrm{L}$ is Planck length.

$$
\mathrm{F}_{\mathrm{C}}=\frac{\mathrm{m}_{\mathrm{ph}} \mathrm{c}^{2}}{\mathrm{r}}(\mathrm{Kgs})
$$

The relation between mass and radius of particles based on action performed on centrifugal acceleration way which is the radius of a particle for 1 second must be Eq. (167) over $\mathrm{F}_{\mathrm{C}} \mathrm{r}_{\mathrm{p}}=\mathrm{m}_{\mathrm{p}} \mathrm{c}^{2}$.

$$
\mathrm{r}_{\mathrm{p}}=\frac{\mathrm{m}_{\mathrm{p}}}{\mathrm{h}}(\mathrm{m})
$$

If this is the situation, then particles must have bigger radius due to Eq. (167) since is not the same with Eq. (164); however the gravitation on themselves during expansion and revacuuming of the space which is already a vacuum in a small time interval prevent it. Considering this it can be said, that the free particles which do not consists of sub-atomic particles with small radius and big mass, emerged earlier at the beginning of time; because the same photon stays at a small radius due to the stronger inner vacuum, and this can occur when the universe was denser. This affects the destiny of magnetic dipoles.

\subsubsection{Length contraction}

As matter can only exist as a density, if formation volume moves that means if mass moves, the existence increases in an unit of time, and it reduces the deviation for each round of 
creation motion during outer space motion of mass. Space object experiences constant potential difference during formation because of the time differences. When wave starts to move in one direction, the formed place of the wave goes to disappearance. At this time, the wave moves to the newly formed space. Namely matter does not wait for to be created. Instead of it, it moves by drawing more space. It is called as Lorentz contraction [4] or length contraction. The length contraction can only occur in one direction because of the resultant direction of movement since this is the nature of all motions.

For a static object namely for an object which has no observational outer space motion and has $\mathrm{m}_{0}$ mass magnitude, the length contraction becomes Eq. (168).

$$
\mathrm{L}=\mathrm{L}_{0}\left(1-\frac{\mathrm{v}^{2}}{\mathrm{c}^{2}}\right)
$$

Eq. (168) for the objects which had been accelerated, and are moving at a speed of v. For acceleration work, Eq. (168) turns into $\int \mathrm{L} \mathrm{d} \mathrm{d}_{\mathrm{v}}$ where $\mathrm{v}=\mathrm{ft} / \mathrm{m}_{0}$.

If acceleration work is done by $\mathrm{c}<\mathrm{ft} / \mathrm{m}_{0}$ where $\mathrm{c}$ is the light speed, the method which was derived as Eq. (67) and Eq. (68) are used for $\int \mathrm{L} \mathrm{d}_{\mathrm{v}}$.

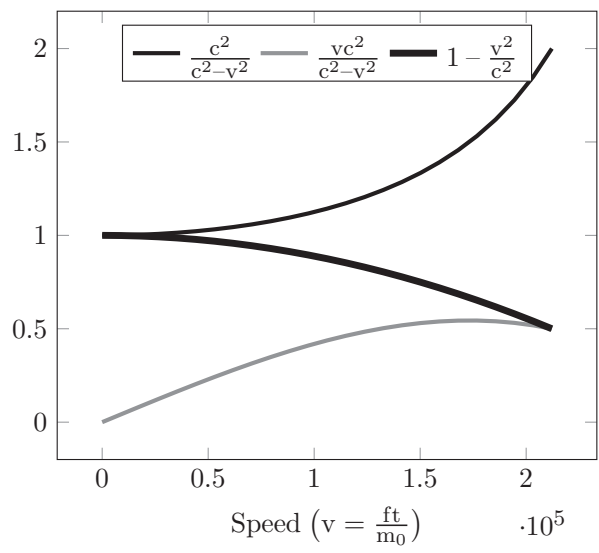

Fig. 34: Mass, length contraction and speed changing graphic from $\mathrm{m}=\mathrm{m}_{0}$ to $\mathrm{m}=2 \mathrm{~m}_{0}$, and $\mathrm{L}=\mathrm{L}_{0}$ to $\mathrm{L}=\mathrm{L}_{0} / 2$

These information contradict with Michelson-Morley experiment [5] in entanglement as the result of the deceleration from infinity since there cannot be an absolute emptiness without motion and energy; because speed of light is creation speed, and it is constant due to limited universe; so it is independent of ether, free space for the status of this experiment. This case is the same for any moving matter in any density. The rays of moving objects are at a constant speed, only their energies are vary. They lose mass instead of change in speed.

If you assume, that particles are perfect sphere, radius of particles and thus their masses are vary due to intensity of the interaction during gravitation. A real decrease or increase emerges for masses; however this occurs according to the relativistic energy transformation formula; because even if gravitation is acceleration, it is like a motion with constant speed for 1 second when masses do not accelerate or have not been accelerated while mutually standing, and it is always $\mathrm{a}=\mathrm{v}$ forever actually for each small period of time in 1 second as well for $\mathrm{t}=1$ second based on $\mathrm{x}=\mathrm{vt}$ as average. Some minor changes in the volume can be observed; however, this occurs as length contraction. $m_{0}$ being the standard mass at the infinite distance from a gravitational field; $\mathrm{m}_{\mathrm{p}}$ being the mass in a planet; $a_{p}$ being the gravitational acceleration on the planet and $a_{S}$ being the gravity acceleration in space; for $\mathrm{m}_{\mathrm{p}}=\mathrm{m}_{0} /\left(1-\left(\mathrm{a}_{\mathrm{p}}^{2} / \mathrm{c}^{2}\right)\right)$, the radius causes the ellipsoid at a certain height from the ground or in space must be Eq. (169), that of course if you assume, that particles are perfect sphere.

$$
\mathrm{r}_{\mathrm{s}}=\frac{\mathrm{h}\left(\mathrm{c}^{2}-\mathrm{a}_{\mathrm{s}}^{2}\right)}{2 \pi \mathrm{m}_{\mathrm{p}} \mathrm{c}\left(\mathrm{c}^{2}-\mathrm{a}_{\mathrm{p}}^{2}\right)}(\mathrm{m})
$$

It starts drawing an ellipsoid.

\subsection{Gravity}

Finite matter gains total energy because of the work done against resistance of the space. This resistance also causes disorder; because matter emerges over time at a frequency. During this process it constantly experiences potential difference because of increasing and decreasing densities. As denser area is more disordered relatively to lower density area, if there exist a mass, it wants to move into lower density from disorder to order. This causes motion and the actual reason of gravity is going from disordered area to more ordered area. Since matter emerges over space as waves being space turns into particles and particles turn into space over time, space lapses into more ordered area, and thus particles which are formed over this sliding space also lapse together with the sliding space.

Aside this, since matter has a circular motion, it creates a centrifugal force; so at the same time that sliding, also potential difference which matter experiences can cause a centrifugal force-dependent motion as a second effect of gravitational motions. This also can be counted as gravity for both ways.

Energy transformations cannot be independent on the equation stated the above as Eq. (65); so as mass of moving object increases, if a mass moves, then the upper limit of mass focus is as much as all mass of the universe; therefore during a gravitational attraction, also the upper limit is total mass of the universe as universe can do work as much as its total energy.

As required by uncertainty, there cannot be an absolute threshold value; so gravity is able to affect from existent the furthest distance at formation speed. If escape velocity is taken, it becomes Eq. (170) over $F x-\int_{0}^{\mathrm{x}} \frac{\mathrm{m}_{1} \mathrm{~m}_{2} \mathrm{G}}{(\mathrm{r}+\mathrm{x})^{2}} \mathrm{~d}_{\mathrm{x}}=\mathrm{m}_{1} \mathrm{v}^{2}$.

$$
\mathrm{v}=\sqrt{\frac{\mathrm{Fx}}{\mathrm{m}_{1}}-\frac{\mathrm{m}_{2} \mathrm{Gx}}{\mathrm{r}(\mathrm{x}+\mathrm{r})}}
$$

The result becomes 0 if both of the sides are squared, and $\mathrm{m}_{1} \mathrm{v}^{2}$ is put instead of $\mathrm{Fx}$. The reason is conservation of energy and entanglement which means emerging with time differences. When the action is divided constantly forever by the parts which are equal to each other, the action performed by gravitation closes to all of the actions performed against gravitation, and this is an evidence for single factor of creation which is gravity. Escape velocity for the land vehicles moving on surface can be calculated with the required setting, $\mathrm{f}$ being gravitational force, based on $\mathrm{mv}^{2} / \mathrm{r}=\mathrm{f}$. A constant state of performing action is required to conserve the velocity; otherwise, the accelerated mass first slows down and comes back even from the existent furthermost distant. Mass and weight are the same thing. Matter has no absolute mass potential independent of gravity.

To be freed of gravitational field or escaping it is not possible.

\section{Inference}

The condition changes only when entered into the another gravitational field which will balance or defeat this situation, 
and an action is performed against the first field temporarily; thus a fixed orbital is not possible. Even if masses, attraction and thus motion are reciprocal, objects get closer to heavier masses around which they rotate, first move away and after get closer or move away because of outer gravitational effects.

\subsubsection{Gravitational motion which is caused by centrifu- gal force}

As there is no mass without motion, for a constant speed which is the result of a limited work done for a limited universe, certainly mass moves on outer space when it is in a different density space. This motion emerges because of centrifugal force which is the result of a cosmic forcing as a result of constant speed work and circular motion.

We know that denser objects move towards gravity in a smaller density; but what is the actual reason of this movement when we think gravity itself on free space. Think that there are 2 rest masses which emerge suddenly and have the same mass magnitude at different distances relatively to each other. When a point mass which creates them by a circular motion emerged, the space which they emerged over it becomes denser because of the movement and the other masses; because as there is no mass without area force, any mass can only be counted as a density; so one of them is counted as it is at the other's space with changing magnitudes by distance. For a fixed speed, if a fixed magnitude assumed basic mass particle as a threshold value passes this space, the velocity it has must decrease and mass it has increase since it has a limited energy as required by conservation of energy and so by conservation of momentum; but as no part of the universe can be independent of the work done of the universe, the mass must get a fixed velocity, and for this condition, velocity cannot decrease; so only mass increases. It means that, at that point which the point mass exists, the density will increase naturally, and this increase can only be by a particle radius decrease. When the point mass increases by starting emergence at a frequency in a closed area which is the formed particle's volume on the denser space, emerging centrifugal force of the mass which the point mass creates it increases; so when the masses emerge at difference distances relatively to each other by the same magnitude, if the density of the other mass on the other one becomes maximum, the other one moves into the denser space way by emerging centrifugal force as this force will increase because of increasing mass during the point mass is passing over denser area.

When the fixed point mass enters a denser area, actually it is waited for that a repulsion must emerge; but it cannot emerge; because space and wave are not independent of each other. Space turns into particle by wave, and particle turns into space in time constantly at a frequency; so for a fixed speed it is always forced to emerge as denser.

Right this point a question emerges that is length contraction effective in formation of atom? As the fixed objects in a gravitational field can be assumed as doing constant speed movement, they must experience a length contraction; so maybe because of the small distances in atom, some temporary particle densities may emerge and emerging centrifugal force of sub-atomic particles may cause different behaviours as a result of length contraction since the shape of the particle will change.

\subsubsection{Conservation of momentum}

If non-flexible collisions are handled, for two objects which move towards each other at light speed, (171) can be written after the collision.

$$
\mathrm{m}_{1} \mathrm{c}-\mathrm{m}_{2} \mathrm{c}=\mathrm{mc}
$$

Here, if it becomes $m_{1}>m_{2}$, then the motion is going to be in + direction; otherwise it is going to be in - direction. For $\mathrm{m}_{1}=\mathrm{m}_{2}$, no motion can occur.

Gravity also moves as waves at light speed like all the other things, that can cause spiral build of galaxies because of emerging latency; because when a mass is created, its mass effect due to distance is only able to emerge according to limited total energy over time. Masses realize this by changing density of space; therefore even if we do not know which function does gravity work over it, by indirect calculations and by using similar functions to the main unknown function that have the same basic logic, for analyzing the condition, (172) can be written.

$$
\mathrm{m}=\frac{3}{4 \pi}\left(\frac{\mathrm{m}_{1}}{\mathrm{r}_{1}^{3}}-\frac{\mathrm{m}_{2}}{\mathrm{r}_{2}^{3}}\right)
$$

Here, for $m_{1}=m_{2}$, if it becomes $r_{1}>r_{2}$, the motion emerges in - direction; otherwise it emerges in + direction. For $r_{1}=r_{2}$, no motion can emerge. For different mass magnitudes, also there exist similar points which motion cannot emerge there. If a third object takes place in here, it does not move; therefore it can be said, that matter is not able to be compressed oneself forever; because there is going to be always an interval at a distance.

Emergence of gravitational motion in this way, is related with space tension. As it was said before, matter is wave which emerges over space by using space. Also creation motion and outer space observational motion are accepted together, and use the same space at the same time; so in changing space densities, direction of the force which is applied to create mass can change, and actually by this way, spin of the mass which the force is applied to create it, can be counted as rotated. Mass moves in the movement direction of the wave has bigger mass magnitude by this way.

Over these information, it can be said, that matter always wants to move from denser space to lower density space; because at that time, space had been stretched enough, and thus the wave wants to be distributed on lower density space by filling it. As it was said before, matter always wants to be more ordered. Already the work which is done to create matter and brings total energy out for it, is repeated in each small period of each small period of time by forcing, and is done against this resistance as well.

The points which cause the biggest displacements are centers of gravity of heavenly bodies; because if attraction between two masses is handled, changing density over distance of one of them on the other one gets the smallest value at the center of gravity of the other one for the longest $r$; therefore, when gotten closer to the point which does not cause motion, emerging attraction and thus motion get smaller, gotten closer to mass repulsion.

Gravitational collisions are counted as flexible collisions when the time differences are ignored; because the equality becomes $\mathrm{m}_{1} \mathrm{v}_{1}=\mathrm{m}_{2} \mathrm{v}_{2}$; therefore, even if different mass magnitudes gain different velocity and acceleration values by the same force, they have the same momentum; thus there does not occur motion after a collision.

As changing densities cause attraction, also cause a mass repulsion.

Over Figure 35, it is not so hard to see the mass repulsion when it becomes $\mathrm{d}_{1}>\mathrm{d}_{2}$. When the environment between the two objects becomes denser, the space lapses into the both $d_{2}$ density space; thus the objects which are created over this space also move away from each other on this sliding space, together with the space. Third or more object can affect the conditions. Sometimes attraction and sometimes repulsion can be detected between objects.

Again, over the similar functions to the actual function, for the objects which are going to push each other, both $\frac{\mathrm{m}_{1}}{\mathrm{r}^{3}}>$ 


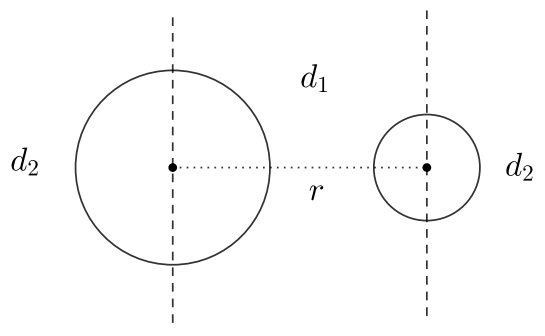

Fig. 35: Repulsion

$\frac{\mathrm{m}_{2}}{\mathrm{r}_{2}^{3}}$ and $\frac{\mathrm{m}_{2}}{\mathrm{r}^{3}}>\frac{\mathrm{m}_{1}}{\mathrm{r}_{1}^{3}}$ inequalities must be provided at the same time; therefore

$$
\frac{\mathrm{m}_{1}^{2}}{\mathrm{~m}_{2}^{2}}=\frac{\mathrm{r}_{1}^{3}}{\mathrm{r}_{2}^{3}}
$$

two objects which provide this (173) values always push each other whatever the $\mathrm{r}$ distance between them; so it can be said, that for example two objects which have the same density always push each other like proton-proton. This is pretty natural; because if a gravitational wave is able to be distributed on deep space over time, it means the outer space density which is smaller than the wave density allows this; so sometimes density of the space between two objects can be assumed as an anomaly since is intersection point of the waves, as higher density; but emergence priority can affect the condition; because a while proton has a mass magnitude, another proton at the other side of the universe can exist in another mass magnitude, that actually both of them have the same mass magnitude for 1 second. In the same manner, different mass magnitudes for 1 second also are able to push each other because of the time differences or emergence priority. They do not have to exist in far places according to each other. Even they can exist in an atom together that you can count some of them as antimatter.

Over these information, it can be said, that there is always a repulsion between objects even if it may smaller than attraction; because, as it was said, different masses gain mass by having different acceleration values of assumed the same basic creative wave; thus different two masses get closer to each other in mass magnitude for different times. This condition always repeats itself at a frequency each second; so it can be said, that there is always an interval at a distance between objects.

For the actual function of (173), it can be said, that the repulsion can be effective on inflation of red giants. Maybe central density causes a repulsion; but if the objects which are too dense and push each other, are formed by more than 1 particle, each particle can be attracted even if the calculation requires repulsion; because there is no absolute bond between particles, and thus total mass density may not behave as onepiece. After a particle flow from one of them to the other one, an attraction may be detected. This is also dependent of initial velocities of heavenly bodies in free space. This can be effective in atom as well.

A side effect can cause repulsion, that if a wave which has high enough amplitude is able to push objects. If you pull a tablecloth off from a table fast enough, the goods on the table almost will not move; because required time for emergence of frictional force will not be provided. Almost there will no time interval for the work done by the friction. In the same manner, high amplitude wave may also be repulsive even during its movement in the direction of lower density space. As to be one of them before or after of the same Ft work as $-\mathrm{Ft}$ and + Ft will cause displacement on space, even if energy and momentum are still conserved at that time, the wave may affect the behaviour of gravitational motions due to characteristic constants of free space. Even it is possible for a single wave which is not in an interaction with other waves, that at that time space becomes like it got a slit.

Matter is able to be polarized by these ways. Waves can boost each other; so if we align atoms side by side, and then if we can rotate their spins by an external effect, then while one side is becoming denser and denser, the other side will be lower density space; but does photon moves in newly emerging or old space direction, since in the both condition, there will emerge the density difference? Maybe it can moves in the both directions as well when a condition is provided. Maybe even it is able to move in the both directions at the same time.

As matter moves from denser space to lower density space, and as gravitational wave must emerge in all directions, then if photon can move freely in space, then free space is the main and natural lower density space; so if we can make a higher vacuum tube, then light can be distributed on space faster than light even if the light speed is still the same at that time, by space tension. You will spend an energy to vacuum the tube for only one time; but even so the vacuum will work each second; so at the end of the tube, generated heat on a plate will be more than total energy of the light before entering the tube. Vacuum is also effective in electricity flow.

\section{Wave monster}

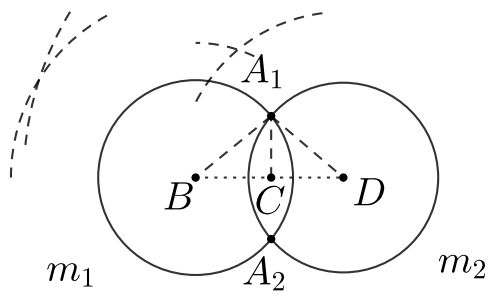

Fig. 36: Gravitational waves

There are a few parts of some gravitational waves which are emitted by $m_{1}$ and $m_{2}$ masses placed at $B$ and $D$ points in Figure 36. The attraction emerges at $A_{1}$ and $A_{2}$ points for this 2 dimensional wave section; therefore over the equality of $\mathrm{m}_{\mathrm{a}} \mathrm{c} \cdot \cos (\alpha)-\mathrm{m}_{\mathrm{b}} \mathrm{c} \cdot \cos (\beta)=\mathrm{mc}$, it becomes $(174)$,

$$
\mathrm{m}=\left(\frac{\mathrm{m}_{\mathrm{a}} \mathrm{r}_{1}}{\mathrm{k}_{1}}-\frac{\mathrm{m}_{\mathrm{b}} \mathrm{r}_{2}}{\mathrm{k}_{2}}\right) \mathrm{j}
$$

where $\cos (\alpha)=\frac{\mathrm{r}_{1}}{\mathrm{k}_{1}}, \cos (\beta)=\frac{\mathrm{r}_{2}}{\mathrm{k}_{2}}, \mathrm{k}_{1}=\mathrm{A}_{1} \mathrm{~B}, \mathrm{k}_{2}=\mathrm{A}_{1} \mathrm{D}$, $r_{1}=B C, r_{2}=C D, m_{a}$ and $m_{b}$ are reduced mass magnitudes at $\mathrm{k}_{1}$ and $\mathrm{k}_{2}$ distances, $\mathrm{j}$ is a constant provides average particle collision amounts of waves since the smallest space parts can collide by different angles or do not collide that can change due to formation speed of light and can take 1 value, smaller or greater values than 1 but 0 .

Assume, that 0 dimensional densities of $m_{1}$ and $m_{2}$ over 3 dimensional densities are $\mathrm{m}_{\mathrm{a}}=\sqrt[3]{\mathrm{d}_{1}} / \mathrm{k}_{1}$ and $\mathrm{m}_{\mathrm{b}}=\sqrt[3]{\mathrm{d}_{2}} / \mathrm{k}_{2}$ where $\sqrt[3]{\mathrm{d}_{\mathrm{n}}}$ is 1 dimensional density over 3 dimensional $\mathrm{d}_{\mathrm{n}}$ density for $\mathrm{k}_{1}$ and $\mathrm{k}_{2}$ lengths. For total $\mathrm{m}$ in $3 \mathrm{D}$, over $2 \pi \mathrm{hm}$, (174) turns into (175), 


$$
\Delta \mathrm{m}=\left(\mathrm{r}_{1} \sqrt[3]{\mathrm{d}_{1}} \sqrt{\mathrm{k}_{1}^{2}-\frac{\mathrm{r}_{1}^{2}}{\mathrm{k}_{1}^{4}}}-\mathrm{r}_{2} \sqrt[3]{\mathrm{d}_{2}} \sqrt{\mathrm{k}_{2}^{2}-\frac{\mathrm{r}_{2}^{2}}{\mathrm{k}_{2}^{4}}}\right) 2 \pi \mathrm{j}
$$

where $\mathrm{h}=\sqrt{\mathrm{k}_{1}^{2}-\mathrm{r}_{1}^{2}}=\sqrt{\mathrm{k}_{2}^{2}-\mathrm{r}_{2}^{2}}$. This is for a single wave. There are $\frac{1}{t_{0}}=v$ times waves radiated from one of them, where $\lambda=\mathrm{ct}_{0}=\frac{\mathrm{c}}{v}$ that is always fixed even if gravitational force changes according to speed in free space of objects even for fixed $r$ length and is important for sub-atomic particles as the lengths are very small relatively to extreme speeds there, and $t_{0}$ from (51). For the distance $r$ between the objects, there are always $\frac{\mathrm{r} v}{\mathrm{c}}$ times waves; so when the attraction is analyzed, a gravitational latency will appear; because mass effect of $\frac{\mathrm{r} v}{\mathrm{c}}$ pieces waves starts to affect each second after $\mathrm{r} / \mathrm{c}$ second; but this actually interests the first expansion of the universe; so if the universe is big enough, still there will be some masses which have not been affected each other.

When the waves intersected, a motion emerges in space; but waves get bigger forever by having bigger radius; so interaction will be still continuing. Right this point, an interesting phenomenon appears, that at the middle distance of two masses, any waves radiated at the same time from both of the masses are collected; because over $\mathrm{k}_{1}^{2}-\mathrm{r}_{1}^{2}=\mathrm{k}_{2}^{2}-\mathrm{r}_{2}^{2}$, the same radius waves can only affect from $\mathrm{r} / 2$ point during infinite periods of increasing radius, since $A_{1}$ and $A_{2}$ will be fixed at the middle for these.

This monster may be effective about half-life period of radioactive elements; because actually there is a time difference between $A_{1}$ and $A_{2}$; thus actually there is a gravitational torque along with gravitation as the objects are going to turn around each other; so maybe when accumulated waves reach enough attraction, as also centrifugal force will increase, neutron may leave atom.

Additionally, how we can draw the wave pattern of free space even if it would change according to mass scatter for changing densities?

\subsubsection{Local gravitational fields}

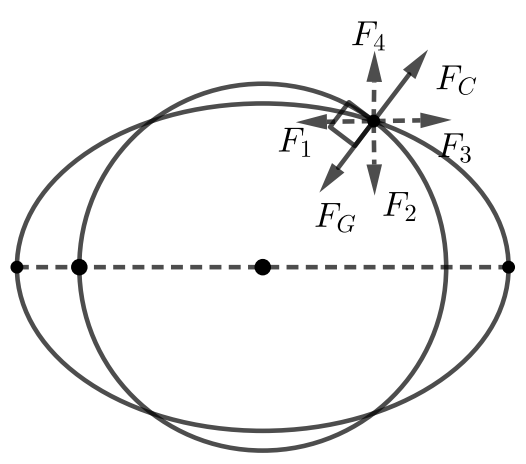

Fig. 37: Circular and elliptic orbits

There is an object which is assumed as doing circular or elliptic motion around a mass on Fig. 37. For this condition, it becomes $\mathrm{F}_{\mathrm{G}}=\sqrt{\mathrm{F}_{1}^{2}+\mathrm{F}_{2}^{2}}$ and $\mathrm{F}_{\mathrm{C}}=\sqrt{\mathrm{F}_{3}^{2}+\mathrm{F}_{4}^{2}}$. Over $\mathrm{F}_{\mathrm{G}}=\mathrm{F}_{\mathrm{C}}$ assumption, it becomes Eq. (176).

$$
\mathrm{F}_{1}^{2}+\mathrm{F}_{2}^{2}=\mathrm{F}_{3}^{2}+\mathrm{F}_{4}^{2}
$$

It means, that for the worst possibility, the components cannot be equal to each other to emerge of motion. If any two of them become equal to each other, then all of them becomes the same force; thus it renders impossible emergence of motion since they are not in the same direction. As a result, we can easily say that it is either $\mathrm{F}_{\mathrm{C}}=\mathrm{F}_{\mathrm{G}}$ with different components or $\mathrm{F}_{\mathrm{C}} \approx \mathrm{F}_{\mathrm{G}}$ again with different components. This conditions render impossible to move on a circular or elliptic orbit; because it must spin being the distance between the object changes over time because of the resultant force even for fixed magnitude different components or for the components which get different values by changing over time, that these two option seems they change escape or falling time and direction; but not. They cannot fly off or fall; because for each attraction or centrifugal force change over $\mathrm{F}_{\mathrm{C}}=\mathrm{F}_{\mathrm{G}}$ equality is conserved; so if escaping force is caused by centrifugal force, at the same magnitude, an attraction emerges. It means, the components change, and an orbit which closes a curve emerges. It cannot perfect circle because of acceleration motion since before a new force is applied with different magnitude and way, the previous force causes acceleration and distance taken.

Orbital objects constantly experience different distances while spinning around a mass. There is no constant boosting. There is a constant transformation between attraction and centrifugal force.

\section{Inference}

Motion cannot be constant speed motion. Objects either accelerate or decelerate constantly or by some changing periods, by using both of them at different times with different magnitudes.

In accordance with conservation of energy, it must be $\mathrm{F}_{1}+$ $\mathrm{F}_{2}+\mathrm{F}_{3}+\mathrm{F}_{4}=\mathrm{F}_{\mathrm{G}}+\mathrm{F}_{\mathrm{C}}$; thus over $\left(\mathrm{F}_{1}+\mathrm{F}_{2}+\mathrm{F}_{3}+\mathrm{F}_{4}\right)^{2}=$ $\left(\mathrm{F}_{\mathrm{G}}+\mathrm{F}_{\mathrm{C}}\right)^{2}$, centrifugal force becomes Eq. (177),

$$
\mathrm{F}_{\mathrm{C}}=\frac{\mathrm{F}_{1}\left(\mathrm{~F}_{2}+\mathrm{F}_{3}+\mathrm{F}_{4}\right)+\mathrm{F}_{2}\left(\mathrm{~F}_{3}+\mathrm{F}_{4}\right)+\mathrm{F}_{3} \mathrm{~F}_{4}}{\sqrt{\mathrm{F}_{1}^{2}+\mathrm{F}_{2}^{2}}}
$$

where $\mathrm{F}_{\mathrm{G}}=\sqrt{\mathrm{F}_{1}^{2}+\mathrm{F}_{2}^{2}}$, and gravitational force becomes Eq. (178),

$$
\mathrm{F}_{\mathrm{G}}=\frac{\mathrm{F}_{1}\left(\mathrm{~F}_{2}+\mathrm{F}_{3}+\mathrm{F}_{4}\right)+\mathrm{F}_{2}\left(\mathrm{~F}_{3}+\mathrm{F}_{4}\right)+\mathrm{F}_{3} \mathrm{~F}_{4}}{\sqrt{\mathrm{F}_{3}^{2}+\mathrm{F}_{4}^{2}}}
$$

where $\mathrm{F}_{\mathrm{C}}=\sqrt{\mathrm{F}_{3}^{2}+\mathrm{F}_{4}^{2}}$. It again seems fixed orbital is not possible.

As it can be seen, here $\mathrm{F}_{1}\left(\mathrm{~F}_{2}+\mathrm{F}_{3}+\mathrm{F}_{4}\right)+\mathrm{F}_{2}\left(\mathrm{~F}_{3}+\mathrm{F}_{4}\right)+$ $\mathrm{F}_{3} \mathrm{~F}_{4}$ must be fixed even for higher dimensions for the same rule, since $\mathrm{F}_{\mathrm{G}}=\mathrm{F}_{\mathrm{C}}$ is the equality and thus denominators change the resultant. Over these, we can write $\mathrm{F}_{\mathrm{G}} \mathrm{F}_{\mathrm{C}}=1$ and $\mathrm{F}_{\mathrm{G}}+\mathrm{F}_{\mathrm{C}}=0$; so gravity becomes Eq. (179),

$$
\mathrm{F}_{\mathrm{G}}=\frac{\mathrm{F}_{\mathrm{C}}+1}{\mathrm{~F}_{\mathrm{C}}-1}
$$

and centrifugal force becomes Eq. (180).

$$
\mathrm{F}_{\mathrm{C}}=\frac{\mathrm{F}_{\mathrm{G}}+1}{\mathrm{~F}_{\mathrm{G}}-1}
$$

For the resultant force it becomes Eq. (181).

$$
\mathrm{R}=\sqrt{\left(\mathrm{F}_{4}-\mathrm{F}_{2}\right)^{2}+\left(\mathrm{F}_{3}-\mathrm{F}_{1}\right)^{2}}
$$


In accordance with Eq. (181), natural motion is circular motion that the difference is too small with regular circle; because $\mathrm{F}_{1}$ and $\mathrm{F}_{2}$ can get very small number near zero; so the orbital acceleration which is because of the components, that even if the component magnitudes do not change over time, the resultant over Eq. (181) causes acceleration over F = ma, causes continuous speed increase in the same orbit without flying off or falling until the existence of the orbital mass creates a density around the central object since already is a resultant of attraction and centrifugal force. When it gets the maximum speed, it means either it became light speed, or since mass emerges for 1 second, its existence is distributed on space and thus speed could not increase to light speed but a different speed limit as attraction properties changed for an unit of time over mass. Hence if an orbital is not circular, it means exactly there is an external drive.

Additionally, it can be seen over real solutions of Eq. (181) that are $F_{1}=F_{3}-R$ and $F_{2}=F_{4}$ or $\mathrm{F}_{1}=\mathrm{F}_{3}+\mathrm{R}$ and $\mathrm{F}_{2}=\mathrm{F}_{4}$. Over these, exactly $\mathrm{F}_{1}$ and $\mathrm{F}_{3}$ cannot be equal to each other but $\mathrm{F}_{2}=\mathrm{F}_{4}$ is equal; so $\mathrm{F}_{1}^{2}+\mathrm{F}_{2}^{2}=\mathrm{F}_{3}^{2}+\mathrm{F}_{4}^{2}$ equation renders impossible to emerge of motion since becomes 0 over $\mathrm{F}_{2}=\mathrm{F}_{4}$. It means equality is not possible and fixed orbits are not possible since it requires $\mathrm{F}_{\mathrm{C}} \approx \mathrm{F}_{\mathrm{G}}$ or $\mathrm{R} \approx$ $\sqrt{\left(\mathrm{F}_{4}-\mathrm{F}_{2}\right)^{2}+\left(\mathrm{F}_{3}-\mathrm{F}_{1}\right)^{2}}$. It means that also perfect circle cannot be drawn by orbital objects. It is only close to regular circle. It is always elliptic.

\section{Warning}

Over Fig. 37, as it can be seen, for example while $\mathrm{F}_{3}$ is increasing $\mathrm{F}_{1}$ cannot increase or if $\mathrm{F}_{4}$ is increasing $\mathrm{F}_{3}$ cannot increase in accordance with Eq. (181) and $\mathrm{F}_{\mathrm{C}}=\mathrm{F}_{\mathrm{G}}$ equality. Resultant force which determines the shape of orbit cannot be fixed since there is an acceleration motion and thus there are changing magnitude components over time. It means, after some distance is taken in the way of centrifugal force, again distance is taken in the opposite way, in the direction of gravity. As the change in component magnitude is continuous, it draws sinusoidal wave on the orbit as a second effect. If is $3 \mathrm{D}$, it draws helix around the central mass.

Every orbital object follows helical path which has circular or elliptic orbit at its center.

\section{Inference}

\subsection{Vibration stopper and reducer densities in space}

Matter is able to work as much as its total energy as total energy means existence; so even if emergence space itself would not lose its energy until the day which the forcing which is done to create emergence area will be removed, particles which emerge over this space have to lose energy; because actually there is no difference between space and particles. Mass is denser space point, and as it has a second potential which is actually entire emergence area has it, it cannot exist forever. The work, which is done to create matter is only done to create free space.

Hence, as denser space will be stopper for a limited speed, for an object which cannot have another high or low speed will experience a friction with space. Even if there was only 1 particle which has no sub particles in space, it would experience friction with space as there cannot be an absolute zero resistance which causes infinite speed for any magnitude force. This cause energy loss as particles have no external energy that as it was said, the work which is done to create matter is only done to create free space. Matter is like a side effect as temporary like a drop of glue which could not be controlled.

\subsubsection{Event horizon}

The most extreme densities of the universe are black holes. There is a limit to be a black hole, and after this limit, if it is not asked black hole's life time, any size black hole can emerge for any radius. In a black hole, event horizon's length becomes Eq. (182) over Eq. (182a) for the work done which is equal to photon's total energy,

$$
\mathrm{x}=\frac{\mathrm{r}_{\mathrm{b}}^{2} \mathrm{c}^{2}}{\mathrm{mG}-\mathrm{r}_{\mathrm{b}} \mathrm{c}^{2}}
$$

where $\mathrm{h}$ is Planck constant, $\mathrm{f}$ is frequency of photon, $\mathrm{m}_{\mathrm{b}}$ is black hole mass, $\mathrm{r}_{\mathrm{b}}$ is the black hole's planetary radius.

$$
\int_{0}^{\mathrm{x}} \frac{\left(\mathrm{hf} / \mathrm{c}^{2}\right) \mathrm{m}_{\mathrm{b}} \mathrm{G}}{\left(\mathrm{x}+\mathrm{r}_{\mathrm{b}}\right)^{2}} \mathrm{~d}_{\mathrm{x}}=\mathrm{hf}
$$

When event horizon length $x$ becomes 0 on Eq. (182a), the result becomes Schwarzschild radius as Eq. (183) over Eq. (183a), with zero event horizon length,

$$
\mathrm{r}_{\mathrm{b}}=\frac{\mathrm{m}_{\mathrm{b}} \mathrm{G}}{\mathrm{c}^{2}}
$$

where $\int \mathrm{d}_{\mathrm{x}}=\mathrm{r}_{\mathrm{b}}$.

$$
\int_{0}^{\mathrm{x}} \frac{\mathrm{m}_{\mathrm{b}} \mathrm{G}}{\mathrm{c}^{2} \mathrm{r}_{\mathrm{b}}^{2}} \mathrm{~d}_{\mathrm{x}}=1
$$

Thereupon it verifies the kinetic energy correction which was explained the above. For motions, because of the creation at the fixed light speed, it can be said that light speed is the highest speed, and the lower is not possible as well. Observational outer space motion and formation motion which is the result of repeated emergence are always together. There is only 1 movement. Matter uses the same space at the same time for any act. The condition does not allow kinetic energy to be like $\mathrm{w}=\int_{0}^{\mathrm{v}} \mathrm{v} \mathrm{d}_{\mathrm{mv}}$; because change amount of a mass at low speeds can be assumed as zero, and the speed always must be fixed and be the light speed; so these two conditions do not allow for integration separately, even if actually they are natural results for each other.

There is one more a kind of black hole, that here, gravitational acceleration of $\frac{\mathrm{mG}}{\mathrm{r}^{2}}$ is equal to the formation speed of c. Over this equation, the black hole's radius becomes

$$
\mathrm{r}=\sqrt{\frac{\mathrm{mG}}{\mathrm{c}}}
$$

Photon loses its energy at the end of 1 second; but this loss does not emerge suddenly.

When Eq. (183) is used on Eq. (182), then event horizon becomes infinite; but if Eq. (184) is used on Eq. (182), then an event horizon occurs, and it becomes Eq. (185). It starts to influence from planetary surface.

$$
x=\frac{m c G}{m G-\sqrt{m c^{3} G}}
$$

Also because of $\mathrm{mv}^{2} / \mathrm{r}$ centrifugal force which is gotten by orbital objects light is included as well, escaping is possible even there will be a deformation amount in the escape time; because an uncertainty of the force line condition will emerge as gravity is calculated from center of masses even for planetary surface of black holes. Repulsion distance 
which is a result of centrifugal force becomes Eq. (186) over Eq. (186a).

$$
\mathrm{x} \geq \frac{\mathrm{mG}}{\mathrm{c}^{2}}-\sqrt{\frac{\mathrm{mG}}{\mathrm{c}}}
$$

where $\mathrm{r}$ is Eq. (184) and $\mathrm{x}$ is Eq. (182). The equation must always be provided at least for $\mathrm{v}=\mathrm{c}$ equation.

$$
\frac{m v^{2}}{r+x} \geq \frac{m m_{b} G}{(r+x)^{2}}
$$

This is an absolute mass repulsion even for light like a hyperbolic perfect mirror. For different $\mathrm{x}$ values which are not related with event horizon between $[0, \mathrm{x}]$ interval, the repulsion can be detected within different times.

\subsubsection{Visible light loss}

Visible light loses its energy in gravitational field, actually other photons are included as well; so a dim occurs for an observational reference distance. If the same energy visible lights suddenly emerge near a planet at the same time, the closer one's loss amount will be more than the further emerging one for the same time; so even if we cannot know all the moves of the universe to determine a certain visible light event horizon, if it is assumed that they started from that place, it becomes Eq. (187) over Eq. (187a).

$$
\mathrm{t}=\frac{\left(v_{1}-v_{2}\right)\left(\mathrm{r}^{3}-(\mathrm{r}-\mathrm{ct})^{3}\right) \mathrm{c}^{4}}{3 \mathrm{f}_{1} \mathrm{~m}_{\mathrm{p}}^{2} \mathrm{G}^{2}}
$$

It is solved by solving equation. It is a fall from $v_{1}$ frequency to $v_{2}$.

$$
\int_{0}^{\mathrm{ct}} \frac{\mathrm{h}\left(v_{1}-v_{2}\right)(\mathrm{r}-\mathrm{x})^{2} \mathrm{c}^{4}}{\mathrm{~h} v_{1} \mathrm{~m}_{\mathrm{p}}^{2} \mathrm{G}^{2}} \mathrm{~d}_{\mathrm{r}}=\mathrm{t}
$$

Over Eq. (187), the visible light event horizon becomes Eq. (187b).

$$
\mathrm{x}=\mathrm{r}-\mathrm{ct}
$$

where $\mathrm{c}$ is the light speed, and $\mathrm{r}$ is the distance from the center of the planet that black holes are included as well.

\subsubsection{Disorder Powered Space Wind: Dark Energy}

If we check the final conditions, and if it is required to make an extended summary, it can be said, that because of uncertainty, matter could not gain infinite energy since the work done for creating matter was not done by infinite energy, since work done is equal to kinetic energy. It emerges in a time interval and at a limited frequency according to the creation work's speed. There is only one work to create all the universe because of an absolute entanglement, and thus any matter in this universe gains energy due to the speed of the work done. Matter uses the same space during any act; so it cannot go faster than light since already it moves at speed of light because of the motion which is caused by the work done to create.

\section{Natural holding capacity of free space}

Emergence in this way renders impossible to move of bodies linear manner since there is no middle point for any force applied because of uncertainty. Any short interval has shorter interval to infinite small part of matter at the same time, even before you measure it. By this way, flexible collision is not possible and thus information is conserved forever since there is not going to be certain destruction during collisions. The amounts get smaller until infinite small amount but it never disappears. Because of this reason, motion can only emerge in circular ways. Actually we can call it a motion which closes a curve; because it is not a perfect circle. Even actually we should call it a motion which does not close a curve because of uncertainty. It is only assumed to calculate it. It does not close a curve; because it is just a part of infinite information. All infinite information is one piece and closes a curve since you cannot add in or detract from infinity as anything is element of infinity. They already existed. It is deterministic. It only appears being in a cause and effect relationship with infinite information.

These also mean, that any mass or energy is only distributed mass or energy because of uncertainty; but the problem is it, that which stringency, frequency, density does matter have on free space because of its existence? What is the reference? Namely, what is the amount of emptiness. As entire universe emerges at a limited frequency, it is not continuous at every point of its free space. Empty space which has no particle or wave also has a frequency. Before emergence of a wave, there must exist free space firstly since particles emerge over this space as waves; so particles cannot have higher frequency than this frequency of free space; because there is going to be no place to emerge after this limit. You must increase the creation frequency and thus light speed or creation speed of the entire universe and thus of the single work done.

As any mass or energy can only be counted as distributed mass or energy because of uncertainty, being $f$ is total frequency of the universe, $\mathrm{V}$ is volume of the universe, natural frequency holding capacity of free space becomes $\mathrm{C}=\mathrm{f} / \mathrm{V}$ for 1 cubic meter. Space cannot hold higher frequency than this in 1 cubic meter naturally whatever the mass scatter is in this volume. If it exists, then space is going to directly be distended. This place then becomes more disordered, and then wants to be fixed by distribution on space, on a wider area because of incompressibility property of matter which is because of limited light speed. Denser space is more disordered; because you need more speed to create denser area since application time and thus speed of the force applied are going to change. Actually more speed is only required after you compressed all the mass of the universe to the old position, then you need more speed and thus the new universe is going to have more energy because of increased speed of the work done since the work done is equal to kinetic energy. Even so you are going to converge to this condition for the densities in the focal points of the universe by some amounts due to density since a stress is going to emerge although you did not compress all the mass of the universe. During this process, for creating denser area with a fixed speed, only disorder will increase as long as you create more and more denser area. If you create a lower density area, it wants to be fixed by an opposite low force; but if you create denser area, the opposite force will be bigger. Also gravity works over this principle. Each gravitational wave creates a potential difference, and when they intersected, the space moves according to the condition that also a repulsion is possible. As matter emerge over this sliding space, also matter moves. Light speed is a gravitational acceleration but an acceleration limit since gravity is the single source of motion; because light always falls during its movement in free space even if is not in an external gravitational field at that time. If you create a potential in space as a wave, it does translational motion in the way of lower density more ordered place which is the movement way, and its extinguishedness time is very long.

This properties may be effective in red giants. If there is not going to be enough gravity which holds mass at the expense of space distension since space can be bended, they cannot prevent inflation. If freely moving alone bodies in medium has low gravitational effects are handled, they are also extremely affected since they have no external support- 
ive gravitational force. Even if you think, that there is only 1 particle in the universe, because of its existence or inner space creation motion which is not observational that actually is the same with observational outer space motion, it is going to move according to spin of the universe; because to be one of them before or after of the same Ft work as $+\mathrm{Ft}$ and $-\mathrm{Ft}$ changes displacement. According to initial movement way of the universe, the particle is going to move by acceleration since it experiences constant potential difference over time on space because of emergence frequency of free space. It always seeks an ordered area but it cannot find. It never stops.

\section{Conclusion}

As the result, free space has a frequency holding capacity for each cubic meter; so initial velocities of moving bodies directly affect own observational outer space constant speed. How many cubic meters they draw per second, this is important. Also their mass and density are important for this drawing. The resistance they experience changes according to these properties. It means, that also different densities of the same frequency light or different frequency lights have different behavior on space. It is going to be misleading if you make the same calculation for different upcoming lights of different size stars. Even red-shift amount is different for more intense light or lower density light or different frequency lights.

Spin of the universe is also pretty important; because as it can cause a deflection on moving bodies, also can cause a resistance or acceleration.

Free objects such as galaxies can be shaped according to the spin of the universe when you assume that they are far enough from a high gravitational area. They can be synchronized with emergence of the space with minimum resistance; so as no object can protect its outer space observational speed forever, also static object which have no other motion than inner formation motion can accelerate. During constantly emerging potential differences, also static objects accelerates and decelerates. This motion is chaotic; because for example it can accelerate 1 second and then 0.2 second decelerates after that $0.1 \mathrm{ac}-$ celerates and then 2 second decelerates. The result can be acceleration as also it can be deceleration. If it is acceleration, it can increase until the space wind decelerates. After that it can rotate or move at constant speed. This is also dependent of the density of the free objects.

\section{Warning}

As stated the above I had derived some properties of the universe over light speed and centrifugal force at the beginning of time, if you assume that the motion is perfect circle that is not actually and there is going to be some differences, such as radius or volume. Additionally I used a little different calculation for Planck constant, Planck length and mass of photon. The results will change in some amounts which are not so different. Holding capacity $\mathrm{C}$ for 1 cubic meter is equal to the following formula over this calculations,

$$
\mathrm{C}=\frac{3 \mathrm{c} \sqrt{2 \pi \mathrm{cG}}}{4 \pi \mathrm{G}^{2}}(\text { Hertz) }
$$

where $\mathrm{c}$ is the speed of light and $\mathrm{G}$ is gravitational constant. It is nearly $5.7 \cdot 10^{28}$ Hertz. Being hf $=\mathrm{m}_{\mathrm{e}} \mathrm{c}^{2}$ where $\mathrm{m}_{\mathrm{e}}$ is mass of an electron, $h$ is Planck constant and $f$ is frequency of electron and $\mathrm{c}$ is the speed of light; it means, that electron has nearly $1.22 \cdot 10^{20}$ Hertz frequency. It means, that 1 cubic meter space can hold only nearly $5 \cdot 10^{8}$ times electrons. It means, that can hold nearly $\mathrm{d}_{\mathrm{s}}=4.5 \cdot 10^{-22} \mathrm{~kg} / \mathrm{m}^{3}$ mass. Over lifting force of liquids equation of $\mathrm{F}_{1}=\mathrm{V}_{\mathrm{s}} \cdot \mathrm{d}_{\mathrm{l}} \cdot \mathrm{g}$ where $F_{l}$ is lifting force, $V_{s}$ is sinking volume and $d_{l}$ is density of the liquid, $g$ is gravitational acceleration that is equal to light speed for 1 second since gravity is the main and single reason of motion as I proved over Heisenberg's uncertainty inequality $\Delta \mathrm{x} \Delta \mathrm{p} \geq \mathrm{h} / 2$ since the inequality interprets change in gravitational potential energy, it becomes

$$
\mathrm{ma}_{\mathrm{o}}=\mathrm{V}_{\mathrm{s}} \cdot 1.35 \cdot 10^{-13}
$$

over $\mathrm{ma}_{\mathrm{o}}=\mathrm{V}_{\mathrm{s}} \cdot \mathrm{d}_{\mathrm{S}} \cdot \mathrm{c}$, where $\mathrm{F}_{1}=\mathrm{ma}_{\mathrm{o}}$, $\mathrm{m}$ is mass of an object such as vehicle in free space far enough from a gravitational field, $a_{O}$ is observational outer space acceleration different than the gravitational formation speed since outer space and inner space motion are accepted and emerge together by using the same space at the same time that when an object moves from A to B it means emergence motion moved, $\mathrm{c}$ is the speed of light as gravitational acceleration here for $1 \mathrm{sec}-$ ond over $\mathrm{v}=$ at since it is the source of emergence speed as gravity for any wave or particle.

These calculations are for macro cosmos to determine a reference; so you cannot say mass scatter is not important in 1 cubic meter for micro cosmos. In the same manner, you must calculate each cubic meter of an object separately. Namely, you must use aerodynamics. For example, while one wing of a satellite is lifting the vehicle, the other one can leave it. You must calculate it according to geometry placed in each cubic meter of the existent outer space object.

\section{Warning}

As a result, for 1 ton object which has 10 cubic meters volume, acceleration or deceleration according to spin becomes

$$
\mathrm{a}_{\mathrm{O}}=1.35 \cdot 10^{-15} \mathrm{~m} / \mathrm{s}^{2}
$$

Alright; but we did not include initial velocity since it draws more volume according to this speed. If you assume, that this object moves at $10 \mathrm{~km}$ per second, for $\mathrm{V}_{\mathrm{S}} \cdot 10.000$, it becomes

$$
\mathrm{a}_{\mathrm{o}}=1.35 \cdot 10^{-11} \mathrm{~m} / \mathrm{s}^{2}
$$

This may explain the pioneer anomaly, and also seems galaxies have lower mass magnitude can turn at higher speeds than heavier ones, that is actually the same with sail effect here. Here electron is only a reference. You can use any particle to determine it. This will not change anything. Also at higher speeds, you must use the energy transformation equation.

Universe gets its volume due to centrifugal force as it was stated the above; but according to emerging particle amount, the volume changes. If particles evaporates in the other name they experience decrease in their vibration in accordance with entropy, then volume increases. This seems like the universe is expanding but not. Also there is time differences along centrifugal way; thus also this causes motion and again can be seen like expanding.

\section{Warning}

\subsubsection{Gravitational Catastrophe and Dark Matter}

It seems that gravity does not work as Newtonian. Distance and gravitational force relation changes over distance. The attraction properties change for each point of free space, 
and have some limits. The attraction changes in accordance with some values between $1 / \mathrm{r}$ and $1 / \mathrm{r}^{2}$ even for the existent furthest or nearest distance.

As we know, outer orbital objects rotate faster than inner orbital objects in galaxies according to our observations. Namely an orbital object must take place in space according to $\mathrm{v}=\sqrt{\frac{\mathrm{mG}}{\mathrm{r}}}$; but it was detected, that $\mathrm{v}$ and $\mathrm{r}$ are not related in this manner. Almost there is no exponential relation like this in galaxy size heavenly bodies.

\section{Attraction magnitude and distance relation}

Eq. (175) is for a single wave. I am not calculating gravity yet. Just analyzing attraction properties. Already at that point, there would emerge no attraction for the same magnitude two waves if you ignore time differences that actually there exists torque; but this is not important. Here over Eq. (192),

$$
\mathrm{Ft}=\Delta \mathrm{mc}
$$

you can calculate the attraction force of Eq. (175) and its way as $(+),(-)$ or 0 being t here is the time which the attraction emerges along a distance at light speed due to the constant wavelength of gravity I derived, and also this is not important now.

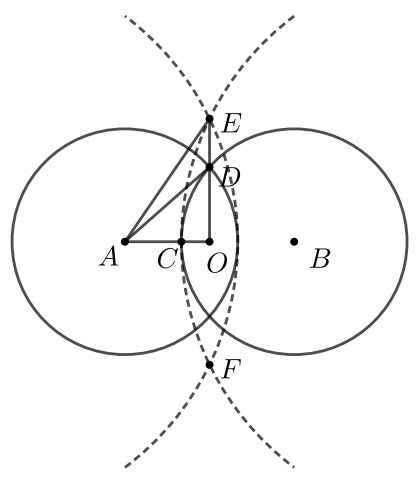

Fig. 38: Another representation of gravitational waves

Now let us handle the Fig. 38. There are two waves radiated from $\mathrm{A}$ and $\mathrm{B}$ points. The bigger wave parts are also radiated from a further distance than A and B by the same masses. Here OC length is always fixed since it is the shortest distance which the attraction emerges along it for the gravitational wavelength when two waves are intersected.

For these definitions, if we remove the constants since they are not inclusive in change being mass is also fixed, and the other wave's values,

$$
\mathrm{F}=\frac{\mathrm{OD}}{\mathrm{AD}^{2}}
$$

the attraction in 3D is basically dependent on Eq. (193) over Eq. (194) which is derived over Eq. (175) without the second wave since is not required,

$$
\mathrm{F}=\sqrt[3]{\frac{3 \mathrm{~m}}{4 \pi \mathrm{AD}^{3}}} \cdot \frac{1}{\mathrm{AD}} \cdot 2 \pi \mathrm{OD}
$$

where $\sqrt[3]{\frac{3 \mathrm{~m}}{4 \pi \mathrm{AD}^{3}}}$ is 1 dimensional density of $\mathrm{m}$ which the mass takes place at A point, $\sqrt[3]{\frac{3 \mathrm{~m}}{4 \pi \mathrm{AD}^{3}}} \cdot \frac{1}{\mathrm{AD}}$ is 0 dimensional density of the same mass.

Since OC is fixed, we can count it as 1 ; so it becomes Eq. (195) over $(\mathrm{AC}+1)^{2}+\mathrm{OD}^{2}=\mathrm{AD}^{2}$.

$$
\mathrm{AC}=\sqrt{\mathrm{AD}^{2}-\mathrm{OD}^{2}}-1
$$

Now if you assume that the same magnitude masses take place at a further distance, for this condition, the partial bigger waves on Fig. 38 emerge. OC is still fixed. Even if it is fixed, as you can see, the other lengths exactly increase, and the rule is determined over Eq. (195). Over Eq. (195), we can easily state, that increase amount of $\mathrm{AD}$ is always bigger than both OD and OA; thus if distance between two the same masses increase gradually, Eq. (193) states, that the attraction decreases. If $\mathrm{AD}$ converges to $\mathrm{OD}$, then the attraction increases.

This condition is not a good news for scientists. It seems, Newtonian calculation can only work in an interval. Not for small distances as also is not for distant places. Already gravitational constant is completely experimental, and it can easily hide the actual functions which the gravity works over them. Namely it remains as faking up. We already must had feel responsible about verifying it by another method. The motions in any universe are chaotic. They repeat in an interval; but you need more repeat and more time to draw the big picture; so gravitational attraction between stars or planets is misleading since is slow. Maybe you can detect some of the anomalies if they are too close to each other like deviation of Mercury. Namely it can be observed like the distance and attraction relation is over $1 / \mathrm{r}^{2}$. For the better, for example electrons which have incredible spinning frequency are perfect references for motion as also the distance is short enough to detect anomaly relatively to Newtonian formula aside high speed.

\section{Time differences}

Everything is not okay. Even became more annoying; because the velocity of the outer orbital objects of galaxies must be much more slower according to these information. Namely if you assume that there is dark matter that there is no dark matter, the dark matter amount must be bigger than the estimated.

If the distance between two objects get bigger, the gravitational torque is going to increase more being more certain and sensible, detectable since gravity emerges at the two intersection points and the points have time differences. Emergence priority causes a potential difference between these points. Namely one of them emerges before and thus causes more attraction than the other one. Between two consecutive emerging smallest part of space, the time difference can be ignored but macro space.

While you are calculating orbital motion over classic method, you assume that whatever the emerging type of the centrifugal force, it must be equal to the attraction force; but it is not true completely. Actually you calculate it over static condition being two objects are standing mutually. Namely if you do not include the centrifugal force, the formula is gravitational attraction in linear way; but according to the above stated method, one of the intersection point emerges later.

If you think one point emerges earlier and state, that $\mathrm{F}_{1}$ torque is applied at this point, being the second torque $\mathrm{F}_{2}$ from the other point, it becomes $F_{1}>F_{2}$. Also it can be said, that energy of the medium namely wave must be conserved. When a space point gets lost since it has an emergence frequency and is not continuous at every point of itself, the energy must be transferred another point. It means, that while a wave is distributed on space over time, it cannot protect its shape. The increasing torque is stolen from the other second 
torque point. Even if again it is distributed on newly emerged space back, since to be of them before or after of the same two opposite Ft work as $+\mathrm{Ft}$ and $-\mathrm{Ft}$ causes displacement in a way (+) or (-) according to which one of them you used firstly even for the same values, it slides. Also rotation namely spin of the universe and thus in which way the masses take place affect the condition. By these ways, linear attraction force and thus momentum turns into angular momentum and attraction. There is no other method and alternative for this as required by conservation of energy. This is not a special phenomenon. The universe works in this way even if some times the result is going to be too small to detect.

As a result, the attraction and distance relation which changes over distance is supplied by the time differencedriven increased gravitational torque; but by which ratio?

\section{Calculation of the time differences}

The universe has an emergence frequency as also it has a spinning frequency as I derived both. I calculated the opposite force which free space applies to moving heavenly bodies in changing ratios according to the density of the objects, that for example Pioneer anomaly can be calculated over this by natural holding capacity of free space; but to make a calculation for the time difference we must know how many space turned into particle.

We must add the extra slowing for the objects like Pioneer since gravitational torque is going to increase; so some of total momentum turns into angular momentum, and so it decelerates in linear way.

Warning

Even if all particles get lost, the total mass, energy and frequency are conserved and thus can be used for such a calculation of the opposite force which is applied by free space; but like this calculation of time difference in galaxies, it is so hard. Actually for free space has no particle, the time difference between each cubic meter of the space becomes $1 / \mathrm{V}$ being $\mathrm{V}$ is the volume of the universe; but if a particle emerges, the density and thus the volume changes. And the amounts are so big. For example for 1 ton object, you need almost $10^{30}$ cubic meters free space according to my calculations; so think that $1 / \mathrm{V}$ is disturbed by this value. You cannot say nothing changes by thinking observational distances that distance does not specify time. It does not work in that way. Space-time is flexible. We must determine some suitable ratios.

Actually it is good, that by the observation, we can calculate the total matter amount of the universe by calculating the deviation in speed and thus by calculating the time difference over this; but even so also we should find a different theoretical method. I do not know yet; but it seems almost at the amount of the distance between two object like Eq. (196),

$$
\mathrm{F}_{\mathrm{G}}\left(\mathrm{r}_{1} \mathrm{t}_{1}-\mathrm{r}_{2} \mathrm{t}_{2}\right)=\mathrm{mv}^{2} / \mathrm{r}
$$

where $\mathrm{F}_{\mathrm{G}}$ is Newtonian gravitational attraction, $\mathrm{r}_{1} \mathrm{t}_{1}-\mathrm{r}_{2} \mathrm{t}_{2}=$ $\mathrm{r}_{0}$, and $\mathrm{F}_{\mathrm{G}} \mathrm{r}_{0}$ is gravitational torque, $\mathrm{r}_{0}=\mathrm{EF}$ over Fig. 38 .

Eq. (196) is only a representation. It is not a work even if it seems like $\mathrm{W}=\mathrm{Fr}$. You can also use it as Ft since the important thing is increasing rotational effect magnitude of the force.

\section{Warning}

\section{The result}

These show us again, that the phenomenon is also chaotic, emerges in an interval in many different kind. These show us, that nothing can move away from each other after a point.
There is a limit for this. They constantly wander around in a limited area. Just there is a flexible interval.

Space objects cannot move away from each other until infinity. This also explains why electrons do not fall into nucleus or fly off; because the phenomenon is not boosting. It is a transformation. Rotating effect of force increases; but this is for protecting the energy which the system holds.

\section{Inference}

\subsection{Conservation of information}

An emptiness because of the reason of absolute energy deficiency is not possible as the reason of deceleration from an absolute energy; thus space allows matter to move and thus emerge at every point of space. This means that all emerging masses are a single mass. When turned back to the beginning of time, then it becomes more understandable; because as it can be seen, there is an emergence by turns, and is a single work. This condition brings about emergence motion around matter itself as required by the single universe mass; but also brings about a perfect entanglement as relativity warned by the reason of masses in an infinite number. Also it can be assumed that, there are infinite numbers of masses instead of single mass by having the same time difference again.

Work can only be done in a time interval by the reason of uncertainty; thus a work is always done against a resistance for any magnitude energy, absolute energy is included as well; so heat and heat differences which emerge because of the work done in a time interval by latency are always together with work. Hence it can be said that there is no kind of energy which cannot turn into mechanical energy as any energy has mass magnitude by $\mathrm{E} / \mathrm{c}^{2}$ (Kgs) mass. If this is the condition, then universe must be the heat which is equal to its total energy, and the heat becomes more certain or uncertain according to the works which are done by using from total energy of the universe.

Emergence velocity must be limited even if the light speed has the general magnitude is not an emergence speed because of the limited uncertain universe, and it must be fixed at last; thus total of the universe cannot be unstable and cannot have an amount of disorder for its total energy and total mass; because nothing is able to be lost from the total energy of the universe. The things which are unstable are masses at focal points of universe; thus universe can turn into a swimming pool without any wave because of lose in mass, namely particle loss in the focal points of universe.

An absolute action threshold value for any action performed in works is not possible because of the one by one emergence as required by uncertainty since uncertainty is only able to exist by increasing or decreasing between absoluteness and absolute absence but its loss. This means, that also each existent mass particle has infinite number of mass particles; thus two different masses never intersect at the same mass magnitude at the same time. This means, that it is not possible a flexible collision in universe, and information is conserved forever as any mutual vector annihilation will not occur. Even the existent smallest influence is going to affect the existent biggest mass potential instantly, and make it move for any long distance, if it is used.

Flexible collision is not possible in universe; in this way, information is conserved forever. There cannot be an action which is not in accordance with this.

Inference

If it was not such that, it would be meaningless to talk about conservation of information; because by an absolute existence, an absolute destruction would emerge. Absolute 
threshold values would change the course of events, and there would be left only information of strong. At the end, would be left the information of the strongest.

Existence of flexible collisions is only assumed as the amount is too small. Information gets smaller forever; but it never gets lost. As to be absolute afterwards is not possible as a result of the requirement of a timeless worker before which was told about it the above, also it is not possible to be lost because of the requirement of to be informed by the infinite, since anything is already element of infinity.

Information and matter must have the same building blocks as the requirement of to be information is having energy. All of information must be at the same place because of the entanglement. Because of the requirement of being decelerated from the infinite, matter must exist as spatial waves over the infinite as it is temporary and thus as it cannot have an absolute part. Recovery of information is possible for any information type; but during the process, the information which is searched for and any information become the same at a point relatively for us but not for the infinite.

As heat is single, also speed is single for matter because of uncertainty and entanglement. Matter has been doing the same motion for the visible outer space motion which is different than the creation and the creation motion; so these two motions are accepted and emerge together; so it can be said that outer space and creation motions are accepted and emerge together, and matter uses the same space during any act. Also during motions, because of the creation at fixed light speed, it can be said, that light speed is the highest speed, and the lower is not possible as well. The condition does not allow kinetic energy to be like Eq. (197).

$$
\mathrm{w}=\int_{0}^{\mathrm{v}} \mathrm{v} \mathrm{d} \mathrm{mv}
$$

It is such that; because the change amount of a mass at low speeds can be assumed as zero, and already the speed always must be fixed, and must become light speed; thus these two conditions do not allow for integration separately, even if actually they are natural results for each other. On the momentum equation stated the above, kinetic energy must be $\mathrm{Fx}=$ $m v^{2}$ for $\mathrm{x}=\mathrm{vt}$. It is not different than a simple multiplication. The requirement for $\frac{\mathrm{mv}^{2}}{2}$ is the same force magnitude even in the smaller periods of time. Namely, the first and the assumed infinite small work must be $\frac{\mathrm{Ft}}{\mathrm{s}}=\frac{\mathrm{m}_{0} \mathrm{v}_{1}}{\mathrm{~s}}$. The second work which is the closest to the first one is $\frac{\mathrm{Ft}}{\mathrm{s}}=\frac{\left(\mathrm{m}_{0}+2 \mathrm{~m}_{0}\right) \mathrm{v}_{2}}{\mathrm{~s}}$ as magnitudes of masses increase as much as used reference time but the smallest slice of time being the same. If the final velocity $v_{f}$ is taken from the equation for the other works, then it will be Eq. (198).

$$
\mathrm{v}_{\mathrm{f}}=\sum_{\mathrm{n}=1}^{\infty} \frac{2 \mathrm{Ft}}{\mathrm{m}_{0} \mathrm{n}(\mathrm{n}+1)}
$$

As the equation is $\sum_{\mathrm{n}=1}^{\infty} \frac{1}{\mathrm{n}(\mathrm{n}+1)}=1$, it turns into Eq. (199).

$$
\mathrm{Ft}=\frac{\mathrm{mv}}{2}
$$

Thereupon it will be Eq. (200),

$$
\mathrm{Fx}=\frac{\mathrm{mv}^{2}}{2}
$$

where $\mathrm{v}=\frac{2 \mathrm{Ft}}{\mathrm{m}}, \mathrm{F}=\frac{\mathrm{mv}}{2 \mathrm{t}}$, and since work done is $\mathrm{Fx}=$ $(\mathrm{mv} / 2 \mathrm{t})\left(2 \mathrm{Ft}^{2} / \mathrm{m}\right)=\mathrm{Ftv}$, for $\mathrm{Ft}=\frac{\mathrm{mv}}{2}$. It is kinetic energy as work done is equal to kinetic energy. Also equation $\mathrm{F}=$ ma must be $\mathrm{F}=\frac{\mathrm{ma}}{2}$ for the same rule. This condition is only possible for a single condition, if work done always has the same magnitude even for the smaller periods of time. There is no formulaic qualify.

\section{Information State of Matter}

Information is not absolute. If you assume, that God created all the information as one by one with the time differences between infinite information, then God must had been created as one by one; because as stated the above, if a work is in a time interval, then an absolute must do the work instead of the finite worker which is dependent of time. As the condition is this, also infinite information cannot be without beginning which is not created as well; because if the information is without beginning, then it must be absolute; but as stated the above, there can only be one absolute who is certain and is not uncertain. Here, a third option point exists which is different than physical facts which form our logic. The third option is not the third option which is different than identity and contradiction of logic philosophy. The third option means, that infinite information is not without beginning together with and as God, and God does not create information one by one as there is no time interval which is God has not known some information yet. Otherwise it is what philosophy of logic says, that this means, that it is if what it is and if what is what known it means no problem.

The third option of philosophy of logic is always impossible. The state of to be true and false at the same time is impossible; because it holds both true and false states, so it depended on the definition of to be true and false. As true and false, even if they are relative, they are opposite to each other at last.

\section{Warning}

The infinite can know space until the smallest detail of space; because information can only be created by the infinite, and information has energy and is spatial. Because of to be known by infinite precision state, all of the infinite information can be located in the infinite small place; thus all of the infinite information does not fluctuate and has no energy. Matter is not created from nothingness; but also it is not absolute as well, because as stated above there is no time interval which God has not known yet some information. Matter was created from imaginary time. The condition can be stated as

The matter which is in imaginary time as information does not fluctuate as also it does not have energy.

\section{Inference}

According to the imaginary time, time is relative and never past, and never will be. Created ones are always at the same place and at the same time; but they are in a state of flow and time gives a reference right this point. The energy that is spent by creatures is $\mathrm{mv}^{2}$ (Js) for a creation speed of $\mathrm{v}$, and they spend the same amount of energy forever or in the infinite sum, since everything is the element of infinity; thus it can be said time travel is not possible as any time is now, and it is scary. As creating past or future time is a paradox for God, it is not possible even for God as well to take an object to the old position if it moves.

Before creating a thing God can only record it as information, due to the potential difference which could occur from the infinite, and can create the thing that God wants to create, even if there is no time actually as required by preservation 
of information. There must be two different record places because of people's choices, at the choices God's different wills and already requirement of conservation of information; but finally all of them must be in a sky book that is formed as matter. As information is not without beginning, creating from nothingness requires this.

Meanwhile information could not be eternal. Create out of nothing first time requires this. Information of God could be seen when asked him in the assumption of everything is known like God; because information could only be created from God himself and it is needed to be eternal to ask questions. Until that time, it seems God does not know, but knows. It seems so, because there is no need to remember or no forgetting issues, due to having timeless and infinite energy. $\mathrm{He}$ does not try to sustain the information all the time. He never works for any multitude big or small work since he will have the same energy like before did work.

According to category of information, everything is not the same thing; therefore there should be a right and a wrong. In any mixture, the basic polar extinction rule of universe has good and bad or right and wrong or true and false division, and there could be no anomalous action. If there is a created one, it could be limited because of uncertainty and subject to a rule, either it is known in an infinite time or not, it has a using manual. Goodness and badness are relative as well. If God was unreferenced infinitely bad, the rule of the created ones would have to be in terms of God's morals; because God, the owner of infinite ferman and discipline could not do the other. It became only one thing because of infinite information that his infinite acts is included to the information as the emerging things are the results. If God was infinitely good, there would be the same; therefore God is always good; because for example to be not able to lift a big weight would seem as bad thing, and you understand that is good also God is good since you need only 1 evidence; but at the point, we can say God can create good or bad because of bad creatures. Bad is in his mind untimely manner; but you created them.

Saying "God does not create the bad" is claims of divinity since nobody has absolute creation power. You cannot say, that these are relative and certain information does not exist; because saying certain information does not exist is also a certain information, that it had already been existed.

\section{Warning}

And soul is God's imaginary blow from himself, and a form of matter since has energy and thus mass. Actually matter is formed shape of soul. There is no exact difference. People sometimes could act against their characters and also change their genesis positive or negative manner; but God knows everything as he has infinite ferman and discipline as he knows all the information timeless manner and became only one thing, and doing things against his own morals which knows them informationally, is a paradox. Because of the imaginary time thus could not be uninformed status, as he knows, that he cannot be he forces himself an imaginary feeling and thinks creatures; but this is a work and creates time, and only possible with creatures. It is other than God himself and is virtual part of God. If God wants to see a real existence, all the creatures lose and God only detects himself. God knows, that what do creatures do and feel; but he does not know how is to be a human being certainly. All of them are together and creatures exist, even if they are not absolute. Creatures seem like they cause permanent damage in God's brain if we assume that God has a brain in real time. This damage is like an absolute damage which could not be healed with physiotherapy even if it is not an absolute damage and is easy to remove by taking them back to information state. The owners of mind has free will even if they are limited and dependent, and the free will is limited as well.

Creation is more than a puppet play; because God's destiny is not being uninformed and knowing the infinite information together with creatures, and the destiny of creatures is being a part of God, even if they could not be infinite and eternal. Already God does not work for any size work since any thing is already element of infinity. Even when he did not create yet, there would no energy difference in infinite energy relatively to creation. Hence the creatures are like self-emerging even for God.

\section{Warning}

All the infinite information is known untimely manner by God; thus destiny is an absolute reality even if it does not interest creatures as it is knowing of God. People must act according to the some known, certain rules. They cannot act according to destiny, that they do not know anything about it. Destiny belief is equivalent of God belief. Furthermore, God is similar to people at the point of longing; because every time is now, and he has not been created everything yet, and is not going to be created all of them one day. People cannot see God completely when they are alive or after death as well. Limited human can see God by parts, and human forgets his old parts. Human cannot combine the parts to understand whatever his memory size. In theory, human can see God by naked eye even in the world; because there is no difference between soul and matter or life and death. Just they are some energy thus condition change.

It is not possible to detract anything from infinite or bring in; because everything is the element of the infinite. As all the infinite information can exist by God, God can only create by his own untimely information. During the process, God's names occur. For example, if creature has hunger property then God gets feeder name. As God knows everything untimely manner as stated the above, he had been gotten all the names which can occur untimely manner. Creatures are like emerged from mold, and creatures can have his all names included the biggest known names like creator as well; it can be said for perfect people if you know the rule, and relatively. God did not create any word for only himself. Existence is transfiguration of God. If humans deny them, they deny themselves and become unbeliever; but human can only create by God's creation. "I'm God" thinking in unity of existence idea means, that I closed to God's morals and have been a limited but clear mirror for him or it means I'm a part of the eternal as an expression. Sky God idea is the same idea and as it is a perfect God belief, if you do not count the corruption, it can only be a prophet doctrine. Sky does not mean God; but it means God in the sky who is in his throne. As there is no emptiness which is without energy because of entanglement, if all infinity is assumed as sky, then Sky Turk or God Turk means Turk who closed to God's morals, melted in God and has been clear mirror for God's names. This is an expression; thus when somebody says sky, then it is not only understood as the sky of the universe. This is as saying "I'm Anatolia" of somebody or saying "I'm Turk" if they are from Turkish land even if they are from other nations at that time. "I'm going to Greek" has the same meaning as well.

As we cannot wait for mathematical calculations from a refrigerator, in the same manner we cannot wait from God for another which does not exist. The unit which does this expectation seems as mind. It seems that mind is an evidence for itself as it is a witness for all thinking, thinking is included as well as the biggest and holy act. It does distinction between good and bad according to the using manual that occurs because of uncertainty. During the action, it becomes grumpy and falls into arrogance when it is in the hands of God like a cattle animal. Arrogance is a mental disease that we must 
run away from it. Mind occurs during interactions between God's names and is the name's interaction healthy, and actually it must be the result of some main names. If it does not work healthy otherwise it becomes arrogance. For example when live name is manifested, a rock can feel like a lamb. When some names as scholar and informed are manifested, a rock can talk to you and can save information on its memory and can make warn for you. Mechanics of mind is logic, and logic can develop as it changes. Different types of lives have the same logic structure amongst themselves in different groups. For example, if you give an order like "Let us climb the tree" for a fish, it says "Do you think this is sensible?" like our usual speeches. Beside it, people know that why they cannot kill people unjustly or sex with their mothers.

As logic progresses over information, it can go backwards over information in the other name go into ignorance as well. A basketball player who can basket from midfield and whose skills increased because of own experience can calculate the score according to own logic. If the player is master, maybe can score without looking at the basket. If you want jump of the player from a balcony over a fence that is lower than the balcony and farther, then even if the player can achieve to jump over the fence, maybe saves own somewhere; but as made complex, also may leave on the fence some of own mind.

\section{Warning}

God can only create his creatures by some potential differences which have to be assumed like matter, particle, and any type of sensed different mass even for souls. Saying "God is able to create the creatures without any reason, cause, instrument." is completely relative. He does not create like us. For example, if you want to teleport to somewhere, you have to pray or you have to make some machines, and also you realize all the act by God's creation; but God only thinks when he wanted to create something without an instrument which is similar to our instruments, reasons; he is not at our situation, condition even if he uses the same space. This reasons for our understandings, and there is an absolute red line even if he creates by some particles; so at this point, Charles Darwin who is the owner of the famous evolution theory maybe did a masterful shot to the target board when even we had not been known anything yet about DNA. As actually God creates by some matter even if his creation is different than our usual ones, he creates them by physical frames with a frequency and the same with computer logic as relativity and uncertainty warned. This can be the same for looked for or sensed situation, condition and it can be said even another situation is not possible.

There is no difference between any organs. They are the same in the computer logic; thus Darwin's methods may be misleading, and the situation may be hyper irrelevant easily as well. Because of this reasons, we must use super computers to understand the formation with any combination.

\section{Warning}

Each action is always together with a probability. As it does not matter for God, that he creates A or B magnitude mass since he is not going to work and thus easy to create, also it is not important, that probability of a phenomenon is $\mathrm{A}$ or $\mathrm{B}$. Whatever the actual value, for any existent small probability, if it is in a probability border line, God can create it at the same difficulty with the highest probability; so as he always create by probability, if he wants to create human like self creation, it only thinks, and it happens at the end of the required time.

\section{Discussing}

\subsection{Wave-particle duality over space}

As it can be seen, as the universe or any universe can only decelerate from absolute space as a part of it, there cannot be an emptiness without energy. Any mass density can only emerge over the emergence space which emerges over absolute space as a part of it as wave which is the result of the time differences as also it is temporary. It is wave; because there will not emerge a mass part which cannot be defined as a density. Any mass is only possible as distributed load that a reference is required to define it.

Right this point, also particles are waves, and any particle must have the same formation speed as there is only single work to create all the universe. Particles' different mass magnitudes are because of acceleration of the same basic wave for the same speed. $m_{p}=m_{t} f$ can give a particle's mass where $\mathrm{m}_{\mathrm{t}}$ is the basic mass and $\mathrm{f}$ is the basic mass's frequency; but actually it is still need more detailed explanation. As it was stated the above, when absolute space deformed, then energy and repeat frequency emerge until to be fixed. It always wants to be fixed if the force which causes the deformation is not removed. During this process, different acceleration wave types of the same wave emerge. Even if the formation speed is fixed, waves which form particles can go faster than this formation speed and so can take more distance for the same time because of the. bigger acceleration caused by space tension; so as frequency is $\mathrm{f}=\frac{\mathrm{v}}{\mathrm{x}}$, for a fixed speed, as the distance taken will be bigger for particles, particle forming one of the same wave will have smaller frequency. This is not the same thing with $m_{p}=m_{t} f$ even if it can be calculated by this equation.

Over the work done to create a particle as $\mathrm{F}_{\mathrm{p}} \mathrm{x}_{\mathrm{p}}=\mathrm{m}_{\mathrm{p}} \mathrm{v}^{2}$, it becomes Eq. (201),

$$
\mathrm{F}_{\mathrm{p}}=\mathrm{m}_{\mathrm{tV}}
$$

where $\mathrm{x}_{\mathrm{p}}=\mathrm{a}_{\mathrm{p}} \mathrm{t}^{2}, \mathrm{t}=1$ second and $\mathrm{a}_{\mathrm{p}}=\frac{\mathrm{m}_{\mathrm{p}} \mathrm{v}}{\mathrm{m}_{\mathrm{t}}}$ over Eq. (133).

For the work done to create a single free wave as $\mathrm{F}_{\mathrm{t}} \mathrm{x}_{\mathrm{t}}=$ $\mathrm{m}_{\mathrm{t}} \mathrm{v}^{2}$, it becomes Eq. (202),

$$
\mathrm{F}_{\mathrm{t}}=\mathrm{m}_{\mathrm{t} \mathrm{V}}
$$

where $\mathrm{x}_{\mathrm{t}}=\mathrm{a}_{\mathrm{t}} \mathrm{t}^{2}, \mathrm{t}_{1}$ second and $\mathrm{a}_{\mathrm{t}}=\mathrm{v}$; therefore the force which is applied to create a single free wave and the force which is applied to create a particle which has bigger mass and energy are the same force. It is impossible if you do not understand it that any force is distrubuted force by $\mathbf{P}=\mathbf{F A}$.

\subsubsection{Higgs Field and Higgs Particle}

Right this point, Higgs Field or actually free space itself as entire universe itself is pretty requried as there is no altenative to create a particle; but the problem is it that, actually there cannot be a mass particle to bring mass out. There is infinite frequecy, and also Higgs needs mass particle. The assumed basic mass particle should be photon.

\subsubsection{Michelson-Morley Experiment}

In the same manner, an ether or in the other name free space is required even for Michelson-Morley Experiment [5]. This experiment says that if there was a matter in free space, it would affect the light; but it is escaped that, actually there is one work to create all the universe; so any element of the universe is only depended on the single work's application speed. Nothing can change this speed as it is formation speed. Only energy can change by decreasing mass because of friction 
with space as emergence area which particles emerge over it always wants to be fixed. For this reason, as particles have no external energy, they lose mass but emergence space's itself. Universe wants to evaporate any particle over it, and after that wants to be fixed. Formation speed or in the other name light speed never changes according to an observer.

\subsubsection{Yang-Mills's mass gap and Ultraviolet catastrophe}

As the first inference for mass gap [6], as it can be seen over the resultant force section, matter can only get a fixed angle to emerge. It means that matter never visits infinite or zero value. Also emergence space provides $\Delta>0$ condition as there cannot be an emptiness without energy because of deceleration from infinite. It means that matter does not visit zero energy point even for imaginary time. Deterministic image appears. Even so, still we must separate the virtual part, and say that matter can visit zero and infinite value that emergence space is included to matter. For the both condition it gets lost, and absolute space which emergence area emerges over it remains before emerging again as it has a repeat frequency.

As the second inference, as it can be seen, as space is absolute, also emergence space which particles emerge over it and it emerges over the absolute space is invariant. Only the things which emerge over emergence space change and actually get lost. Also it was there before the beginning of time as imaginary which means is not absolute but also like not created even if it was created. It was created like anything; because nothing can be absolute afterwards as matter is absolute inert. An absolute must work instead of any worker; but as everything is element of infinity, also nothing can be created afterwards. This creates an imaginary time.

Because of the same reasons, as any work is done one by one in a time interval, also radiation radiates one by one without visit infinite or zero values. For example if you assume that a wave forms a particle by spinning around a circle, for a calculation method $\tan (\alpha)$ never visits zero or infinite value. It emerges by infinite small motion and these points are always deferred. Hence, the problem which made Planck busy [7] is his solution by a frequency and actually by a mass and so energy gap.

\subsection{Probability and the strange cat of Schrödinger}

Because of emerging uncertainty, as it was said, matter only can be defined as a density; so it cannot be said that matter is there.

If a second system emerges different than creation, like atom which electrons spin around it, if speed of the electrons increases, then density of the electron around the atom increases; but it will be distributed around atom. It can only be said that $\mathrm{x} \%$ of the mass there and $\mathrm{y} \%$ of the mass is there. As the creation is in a time interval, if emergence motion moves around an atom, as formation motion and outer space motion are accepted and emerge together, formation will be distributed around atom as well. After that, it is like emergence around atom in different density. Even so, probability is not an obstacle against determinism. It does not mean anything is random.

As the strange cat, at is dead and also is alive point, information is prepared. Information always exists in both states; but as nobody can know an information untimely manner aside infinity itself, the work becomes resistance with a heat for the infinite creator because of time, and the information is prepared and is created one by one.

The wave equation of Schrödinger seems a general representation. Namely it seems like an attractor. It does not give the actual shape of atom. Even, if you accept electron as wave, it is very dangerous that math may interpret the creation frequency as electron is there and here being there is absolute emptiness between the places since matter is not continuous. Namely, you know that number pi has all credit numbers and passwords; but if you find the one which belongs to someone, you must investigate the conditions which cause the number. The same may be valid for field equations. Namely you can make an inference like black holes over the geometric rules of math by field equations; but do not forget it is like an intersection point of any probability. It is like a break point. These seem only for motivation instruments about what is going on there to draw a road map.

\section{Warning}

Actually time differences and infinite frequency are a progressive explanation of the Copenhagen Interpretation; because as long as there is an interaction element that you can call it as observer, matter emerges. At the times when a relation appears, matter appears together with the relation. Observer does not have to be living thing. Any point of free space has emergence priority and thus has different speeds at the same time since constant speed is not possible as it was proven. There is only one work to create all the universe, and any point gets the same speed of the work-done to create universe for 1 second of each space point, not at the same time. Each point is counted as observer. The light speed is not fixed for any reference system at the same moment. Also it can slows down that actually this must be the actual expectation of change in time. Namely speed does not slow; but is a result of distribution on area. The creation work is over area, and the work is scanning. Speed must be understood in this manner. Namely, if observational speed slows, it means there is more area in the medium to scan, that there is denser space part. At this condition, you close to the smallest creation time.

As will power, it has a second effect on matter; because natural flow of motion is dependent on some natural physical rules; but you can change the natural flow by will.

As a result, matter has no absolute threshold value. Any small force can move any big mass instantly. At the moments when a measurement is done or an interaction emerged, matter is certain as it converges to a number. It appears from imaginary time that all infinite information is there without motion, does work and gets smaller forever as irrational value by converging and under the estimated threshold value for a limit. Infinite frequency condition is this. Otherwise matter could not appear with infinite frequency. This is the explanation of the motion which does not repeat itself even in a limited interval. By this way, information get smaller forever but its loss, and constantly converges to a distance value forever. You think it stooped but not. Like the sea waves, it comes ashore as waves even in free space under the estimated threshold value as required by conservation of information. There is no flexible collision because of the time differences. At the same moment, any point has different mass, time and velocity.

\subsection{Singulartiy}

As matter has an incompatibility feature as stated the above, a singularity which renders possible infinite magnitudes cannot emerge. Already, as denser area will be more vibration stopper than non-intensive area, a singularity must be lost before it has not emerged yet.

\subsection{Time travel}

According to the imaginary time, time is relative and never past, and never will pass. Created ones are always at 
the same place and at the same time; but they are in a state of flow and time gives a reference right at this point. The energy that is spent by creatures is $\mathrm{mv}^{2}\left(\mathrm{Js}_{\mathrm{S}}\right)$ for a creation speed of $\mathrm{v}$, and they spend the same amount of energy forever or in the infinite sum, since everything is the element of infinity; thus it can be said that time travel is not possbile. Anytime is now.

Additionally, gravity is like general motions; because it causes acceleration. Even if the masses which are under gravitational force effect do not move, acceleration increases their mass. For example, If gravity is high for a fixed object in this field, it is like constant acceleration motion in free space; so let us assume for the moving objects, time passes faster or slower somehow. As gravity is like constant speed movement and brings momentum and thus weight out to masses, somehow also let us assume that gravitational areas affect the way of time flow in some directions as next or back. In the mix, in any condition, as time is the displacement duration which occurs together with energy, without doubt it must affect the energy of matter. If time decreases, energy decreases. If this is the condition, then for back time travel, matter gets zero energy as time was slowed down and finally became zero; thus matter loses its total energy for the next move. Also zero energy is not possible because of imaginary time since everything is element of infinity. Crossing or jumping from zero point is not possible in next time travel move.

Also again for these conditions which are moving objects or big gravitational fields, if it is chosen one of the options of time passes faster or slower for back or next time travels, we need a gravity which must decrease and a gravitational field which we must stay away and take place at infinite distance from it. As faster free space moving objects and the fixed objects which are in strong gravitational fields are assumed as the same because of nature of gravity, in any mix, we need increasing gravity; but also at the same time we need decreasing gravitational force. This is a paradox. All of these things sign to a now; thus neither back nor next time travels are not possible. If you want time travel, then space must be lost and then there will be no next even if it is past or future. As creating past or future time is a paradox for infinity, it is not possible even for infinity as well to take an object to its old position if it moves. Space and time are not independent of each other; so time travel is not possible.

If you handle a relative time travel, it can be said that it is possible. If you handle two different thust engines which have different thrust force, time passes faster for the faster one relatively.

\subsection{Pioneer anomaly}

As matter is uncertain which emerges by a frequency, certainly because of the repeat, there will always emerge a centrifugal force even if it is not a perfect circle. Already perfect circle is not possible because of the time difference, and because of this reason, one day The Sun will rise from the west relatively to our eyes. It is a spin effect like an endless retro movement. The world's spin will change, and it is possible for any particle as well. Think, that a sphere spinning on $+x$ and $-\mathrm{x}$ axis. While it is spinning think, that also it is spinning much more slower on $+y$ and $-y$ axis.

Every motion has minimum two components on space with or without reference. Linear motion is not possible. The actual reason is gravity which is dependent of the time differences on space. Possible emerging smallest potential difference suddenly causes gravity and thus motion. Gravitational torque and the other properties of gravity become active at that time. As you can see over the above stated gravitational calculations in Gravity section, also gravity has two components which has different attraction or repulsion force even at the same time.

\section{Warning}

This is a result of the time difference and a single motion. The motion is like the motion of Mercury around The Sun. An motion can only emerge by this way. Even if an observational linear movement relatively to eye is handled, it must has minimum two different components. If there were no star in our system, you cannot understand this spinning; because you will see that it is spinning around itself, and will see that because of the nature of all motions, as a resultant, it only spins in one axis as you have no reference.

As the universe has been emerging by a circular motion like this and is also not absolute, the wave which forms a particle or moves in free space free manner absolutely must move due to rotation way of the universe. Even if the rotation was not constant in a single direction, changing direction of the force applied of the space as particles emerge over this space by the force applied of the space, would cause a displacement which emerges in a single direction due to the initial movement's direction; because to be one of them before or after of the same two Ft work as +Ft and - Ft change displacement; so space stuff always deviate, and also light cannot come here after a distance. There can easily emerge some bright points which a darkness connect them to each other in universe when we looked at the universe from the outside. Light comes by different angles after a distance and it can be easily detected. Spatial frictions also will be effective in red shift even if there is no other mass in space; because light lose energy thus mass and frequency as speed cannot decrease.

\subsection{Different feature particles}

Matter evaporates. There is only one work to create the universe that is only for free space. Only free space is invariant. As particles have no external energy, they evaporate, and is more in denser area. As speed cannot decrease, instead of it mass and total energy of particles decreases; but total energy of the universe is conserved. Only density of the universe changes. Also as any work is in a time interval, when a mass moved in one direction, its mass effect does not become 0 , and always decreases forever; so during a circular motion, it reaches itself at an amount, and it causes evaporation in the other name decrease in vibration again. It cannot be healed. You increased entropy. It is irreversible. This feature of matter can be healer or dangerous for health; because different gravitational forces cause different mass particles even for the same type of elements. For example $\mathrm{D}_{2} \mathrm{O}$ is also water; but it is toxic. Namely, this will change total energy and so attraction properties. Even small changes may cause for example delay in radioactivity by compounding, or may have different affect. The same element does not have the same features if they were in a different gravitational force because of frictions. It can be realized as artificial in particle accelerators; because if mass increases, evaporation increases, and when it stopped outer space motion, it can be detected. There are too much possibility that can not be counted to create different energy the same particle.

\subsection{Black-body radiation}

The matter emerging at the light velocity performs each action at the light velocity as it responds to each effect at the light velocity as well. For example, when a fire occurs, the increasing vibration together with the temperature can affect another particle by friction which is caused by the particle's creation frequency in the gamma frequency; thus as it can be variable, matter can perform black body radiation at visible light frequencies even for the smallest period of time and turn into lower frequencies quickly at the same velocity according to the particle's place of existence and to its type. 
Because of this, pressure causes heat for any matter, and if you can enter the resonance with a particle which is radiating at the same frequency at the reverse direction by magnetic field, you can cool it according to changing vibrations in time as well. We do not need to heat materials if we have enough pressure, and we can use the heat for constant energy resource. Some strong bonds and crystal structures can cause abnormal heat at small amounts as well.

Black body radiation must be the natural vibration of matter and renders atom as a fire ball because of the surface photon in the other name wave, and is induced by its emergence motion which changes with temperature; because matter always together with the heat which is included to total energy; thus also matter cannot reach absolute zero; because velocity and thus energy become zero.

Transparency may be dependent of the natural vibration as well together with electrons and atomic structure. External energy depended color which is caused by the sun or house lights etc., internal energy color which is caused by external heat and called as black body radiation and lasers which emits light because of change in orbital energy are completely different things even if there is an intersection. During the black body radiation, since matter can only use the energy which radiates from its total energy, E being the radiation energy throughout the radiation, its frequency being $v$, and for $\mathrm{E} / \mathrm{c}^{2}$, matter experiences

$$
\mathrm{m}=\mathrm{h} v / \mathrm{c}^{2}(\mathrm{Kgs})
$$

of decrease in mass thus experiences increase in its radius and volume. Considering this information, it can be maintained as

Black body radiation is natural vibration. It causes decrease in mass and energy at the same proportion with the radiation energy throughout the radiation.

\section{Inference}

At that time the matter can be polarized, and an electron flow by the other expression an electron jump which is the same with lightning least between two atoms must emerge in a correct electronic assembly. Also black body radiation can be a factor for photoelectric effect. If the extra radiation from outside is focused on a particle as a laser beam like in laser fusion systems, since matter can perform action to the extent of its total energy and since vibrations will be deemed motion, it turns the matter into photon by vibrating in the emergence frequency according to the relativistic energy transfer. Length contraction occurs based on the mean motion during that time. Vibrations are accepted as relative motion; thus the transformation is done by the relative transformation of energy equation which stated the above for hight speeds.

I mean with increasing frequency visible light frequency which increases together with temperature increase. I do not mean increase in creation frequency. Actually, temperature-based increase causes vibration on creation movement. Namely, current order of matter is disrupted; so as creation frequency is the same, also it affects another particle more since amplitude of each turn is going to increase because of the disruption in order.

Warning

\subsection{Coulomb barrier}

There is a representation of magnitude change over distance in gravitational waves which are emitted from A point on Fig. 39. Here, $\mathrm{E}_{2}$ area is more ordered and thus attraction is detected from $\mathrm{C}$ to $\mathrm{B}$. An object which is getting closer to A point by gravitation. $\mathrm{E}_{1}$ area is disordered and thus repulsion is detected. Force $(\mathrm{F})$ and distance $(\mathrm{x})$ are dependent of the graph. The sin functions are only representation. The magnitudes can be different than actual event.

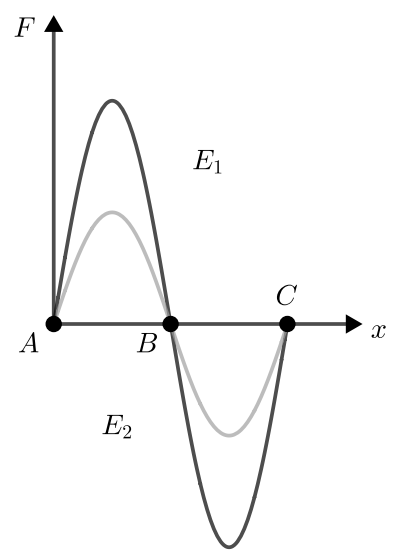

Fig. 39: Near gravitational waves

As you can see, for $-\mathrm{E}_{1}$ and $+\mathrm{E}_{2}$, total gravitational force inequality becomes Eq. (204) where $\mathrm{AB}=\mathrm{x}$ and $\mathrm{BC}=\mathrm{x}$ since gravitational force is related with square of distance.

$$
\frac{1}{\int_{0}^{x} f(x)}>\frac{1}{\int_{x}^{2 x} f(x)}
$$

This means the inequality is always Eq. (205) for their absolute value.

$$
\mathrm{E}_{1}>\mathrm{E}_{2}
$$

Right this point, let us compare that two different waves which have different amplitude on the figure. Higher mass magnitudes cause higher amplitude wave since any gravitational wave has the same wavelength and speed; so due to this, the attraction properties change. For the lower amplitude wave, from $\mathrm{C}$ to $\mathrm{B}$, required energy is $\mathrm{E}_{12}$ and also from $\mathrm{B}$ to $\mathrm{C}$, is $\mathrm{E}_{11}$. For the higher amplitude wave, they are $\mathrm{E}_{\mathrm{h} 2}$ and $\mathrm{E}_{\mathrm{h} 1}$. For this values it becomes $\mathrm{E}_{\mathrm{l} 1}>\mathrm{E}_{\mathrm{l} 2}$ and $\mathrm{E}_{\mathrm{h} 1}>\mathrm{E}_{\mathrm{h} 2}$; so also it becomes Eq. (206).

$$
\mathrm{E}_{\mathrm{h} 1}>\mathrm{E}_{\mathrm{l} 1}
$$

Eq. (206) states, that if mass increases, then the distance between two masses which are placed closer increases. When you exceeded the barrier by an external enforcement, after that the barrier push the mass in the way of $-x$ not $+x$ even if the mass wants to be go out when you think the gravitational wave is radiated in $3 \mathrm{D}$, in all directions. Namely it is imprisoned.

When you pass the barrier over, you are imprisoned; because the last impulsive barrier wave starts to impulse in the opposite way when it emerged. Also after this wave, there is an extremely low density space which causes attraction that is bigger than the impulsive barrier wave; but you cannot progress more; because after this attractive wave also again more powerful impulsive wave exists. Namely you are imprisoned between two impulsive wave in a attractive area. 
Warning

\subsubsection{Nucleosynthesis}

Coulomb Barrier states, that the smartest way of nucleosynthesis is using light masses to combine; because as you can see, heavier mass causes more powerful repulsion. Namely, if you combine a nucleus has two particles, the emerging mass has lower repulsion than 3 particle nucleus; so you must create bigger nucleus by order, by sending light mass particles. It is not the best way colliding two elements to create a heavy element if you do not know how the elements are made. Displacement is important and is effective halfperiod of radioactive elements. You can detect different version of radioactive elements. This is dependent of how they are made, and where, in which gravitational area since gravity causes evaporation. Even all these are also effective during producing composite materials. Timing, placement, heat and shock change everything. For example, temperature is very important; because mass decreases as long as matter radiates; so also repulsion and thus fusion energy and force decrease. If you send too fast, it might not be suitable as also the slow one will not. Cooling time, heating time are also important; because the gravitational wave has very short wavelength as it was proven the above. It is nearly $10^{27}$ Hertz. Even to be one of them before or after of two the same opposite work as $+\mathrm{Ft}$ and $-\mathrm{Ft}$ which are applied to the same mass after the same $\mathrm{t}$ time creates displacement and changes displacement way; so think, that the particles of a nucleus moving back and forth for each wavelength when they are imprisoned namely when the movement in the ordered way is smaller. Even if one day a fission is going to emerge, synthesis method and the condition of the environment can affect the time as also can affect the formed production.

This phenomena also can explain the soil formation of planets. The same effects can be detected in atomic bonds and crystal structure. For example metal fatigue cannot be repaired. You must melt and compress again. It is also possible for any type composite production.

\section{Warning}

Length contraction and centrifugal force are also effective since gravitational attraction can be counted as constant speed movement. If length contraction occurs that it occurs if particles are too close, then centrifugal force affect more.

You should try different variations of materials for nucleosynthesis. If you accelerate a particle, its mass increases and thus also its life time decreases because of the merging evaporation in the other name vibration decrease because of the emerging denser space since particles have no external supporter energy; so by this way when you stop the acceleration, the accelerated particle is going to get different and lower mass magnitude. It means it has different attraction properties as also it is going to be a good nucleosynthesis material which has low mass magnitude.

Also you should try to get the perfect timing. For this, for example, you can send a bigger mass after sending a lower mass almost without a time difference.

Because of the repulsion rules, the mass scatter of the nucleus is not homogeneous. From center to surface, the distance between each consecutive mass group has bigger distance like the distances of the orbitals of electrons around atom. Electron orbitals also have different distances because of this reason. Even the universe is like this even if it may not seen like an atom.

Warning

\subsubsection{Expansion of stars}

Over $r=\sqrt{\frac{\mathrm{mG}}{\mathrm{c}}}$ which is the black hole radius does not let light off and gravitational acceleration is equal to magnitude of light speed for 1 second there, assume that $m$ magnitude mass takes place in the heart of stars for a radius. You can easily see, that there is certainly black holes or in the other name black masses in the hearth of stars. Namely, observed brightness is not actual brightness. Light cannot travel to the surface; so also actual temperature decreases for the surface since light can be counted as temperature as it is the source of heat energy. If vibration decreases, then it is going to be colder. It does not have to be a black mass or black hole. If the values get closer to a black hole with the above stated radius, the same effects are detected more certain.

Because of this reason, when you counted denser material is produced by fusion, actually it causes density decrease of environment which it takes place in it for the same radius since it needs more space. Even the production is denser, it cannot take place in the same volume; so for the same radius, actually mass density decreases; thus the black hole radius decreases; so as stars start to expand as long as they produce denser material by fusion over time slowly, also they get brighter since the central mass let more light come out. Namely, the increasing brightness is appearance of current, hided light and temperature even while star is cooling and dying. After a point, you star cannot compress as also cannot heat more, and the fusion reaction stops. As compression force decreases over time, also temperature decrease as a second effect even if you need both of them as increasing for fusion of heavier elements. Namely, for example Carbon needs more temperature and enforcement than Hydrogen.

Nuclear reaction may not be realized by order. For example, if you create a potential difference has enough energy to ionize the air between the sheets in a closed environment, there will be a latency respectively to the calculation; because the scatter is not perfect. Like lightnings, almost randomly there will emerge a potential difference over different atoms placed at different points; so some of the energy will turn into kinetic energy instead of ionizing another atom. This will continue by increasing as long as ionized atom number decreases. In the similar manner, even if there will emerge Hydrogen fusion more, for example also Carbon can be created together from Hydrogen.

\section{Warning}

Namely, same density particles push each other as it was said at the gravity section when you ignore time differences between masses since is a very very low amount for star system size. You can assume, any point emerges at the same time for such a size. They push since they have the same amplitude wave. A potential difference cannot emerge. Even for different density particles, after a point, again a repulsion force is detected if they get closer enough; because each effect is in a time interval; so if you push an object and then suddenly attract it, the displacement changes according to time difference between the two opposite works. When the different amplitude waves are intersected, both spaces moves in the lower density area; but then also denser and repulsive density emerge during transition at a point since space and object experience potential difference over time. Since new emerged waves have bigger amplitude and energy as they have not been distributed on space yet, the force which causes sliding into lower density is going to be smaller then the repulsivity which emerges immediately after this sliding before a new sliding has not emerged yet, is closer the emitter object. For these different density objects, also you must need exter- 
nal enforcement for fusion like in fusion of the same density objects. As amplitude is directly related with mass, also the same density particles which have more mass magnitude have higher amplitude; thus also repulsion is bigger.

Already denser area is more disordered; thus it wants to be ordered more by distribution on lower density space. As higher amplitude and thus higher disordered masses creates high repulsion force, also they create higher ordered and thus much more lower density space; so also repulsion is detected greatly increased since increased repulsion wants to be more ordered faster by going into much more lower density ordered space as a second effect aside repulsion force itself.

Warning

Also it seems, that there are some places around stars which is hotter than the surface because of boosting of gravitational torque which is a result of total mass. This torque has the energy which can reach light to light speed again. This may create a second spatial thermal radiation which has no spectral character like elements, and caused because of stress.

\subsubsection{Faster displacement of galaxies}

If you apply the rule of the barrier to galaxies, it can be said, that after a time, denser mass density becomes lower density than lower mass density since they have more repulsion. It means, that they rotate slowly since opposite space force over Eq. (189) is going to be bigger even if denser space object are affected less than lower density objects. You cannot keep all mass in a required volume even if the denser mass density has bigger potential to rotate faster. It never appears.

Also if there is a big mass density, this mass can easily distribute own stuff to farther places because of emerging centrifugal force; so the mass scatter density which emerges after the distribution is going to be lower relatively to lower mass density. It also means, that big mass densities rotate slowly since opposite space force over Eq. (189) is going to be bigger even if denser space object are affected less than lower density objects.

\subsection{The laws of thermodynamics}

In accordance with classic mechanics, only the third law is false. Efficiency can get values greater than 1 since it is possible to make matter worked by using its own energy. This is like turning a switch on of a factory. In this factory, there is a system assembled and also there is an energy potential currently on the line. You just turn the switch on. As matter, the potential on the energy transmitting line can be counted as total energy of matter. If you make the required assembly, also you can produce free energy by only switching.

\subsubsection{Thermal equilibrium}

In accordance with quantum mechanics, there is no thermal equilibrium. The equilibrium occurs approximately because of the time differences. Any point of the space has time difference namely has emergence priority; so any point has different mass, time and energy as also has different temperature.

\subsubsection{Conservation of information}

In accordance with quantum mechanics, information can neither be created nor destroyed; but for both, there are some special condition like imaginary time and to be able to create in infinite precision. Nobody was able to get a right to choose aside God or infinity itself. The information is it that anything is created by God; yes but when? If you say that created afterwards, it means there is a time interval which God or infinity does not know information, then where did the information come from. You cannot detract from or add in infinity since anything is element of infinity. There must be an imaginary and thus timeless creation of information. After that real time and thus the body of the information appears in 3D. As destruction, it is easily possible for God, that it is taken back to the imaginary time by a collision with infinite precision since anything element of infinity and thus since colliding masses get closer to the same mass magnitude or has infinite density which stops the vibration. No fluctuation, no wave, no time difference, no mass and no time; but you know everything is there. They are only imaginary have no effect. Already any point of infinity or God itself knows any created thing. It means, God uses all infinite energy he holds from the existent smallest work to the biggest work since any point is driven, and any work is at the same difficulty. He does not work for any work since anything is his own element. Even while he was not creating, there would be no total energy change of infinity.

It is impossible to be absolute of matter; because it can only be created by potential differences which are sources of motion and thus energy, mass, time, distance and velocity of matter. As it was proven at the beginning of the article in the Progressive Image section 2.1.2, if there is a limited energy, then it means emerging matter can only be compressed or expanded between limits. Even if faster than light travel is possible at light speed by warp drives, you cannot warp space for further places by the same amount of material even if you has enough energy theoretically. This also means, that there is no infinite variation of one thing as also there is no infinite universe currently exist. By this rule, there exist infinite different things. Already, matter gains its total energy by enforcement of the work done of the matter against the space wants to fixed again. this cannot be randomly. Enforcement cannot be randomly as also cannot be emerge during there exist infinite variations currently. You cannot create infinity by performing a sum operation. If you say that anything exist at the same time, it is only possible when no real time is created.

\section{Warning}

\subsubsection{Entropy}

In accordance with quantum mechanics, as matter emerges by enforcement which causes total energy, that otherwise there would no matter, it is always together with heat and temperature; so as temperature approaches absolute zero, the entropy of a system approaches a constant minimum, and at absolute zero, there is only imaginary time without motion and thus heat.

Matter has entropy which is equal to its own total energy firstly; because it has frequency and the frequency emerges according to recovery wish of space. Namely it exists because of the claim not to exist. Secondly, it has an on-space entropy as a side effect.

If we check it again, emergence of matter is like it, that mass itself creates itself because of conservation of energy; so $\mathrm{mc}=\mathrm{m}_{\mathrm{x}} \mathrm{c}+\mathrm{m}_{\mathrm{y}} \mathrm{c}+\mathrm{m}_{\mathrm{z}} \mathrm{c}$ equation is always provided. Here, even if the three components always change, sum of them is always conserved; therefore the equation becomes Eq. (207),

$$
\mathrm{dx}\left(\frac{1}{\mathrm{dy}}+\frac{1}{\mathrm{dz}}\right)=-\frac{1}{2}
$$


where $\mathrm{ds}^{2}=\mathrm{dx}^{2}+\mathrm{dy}^{2}+\mathrm{dz}^{2}$ and $\mathrm{ds}=\mathrm{dx}+\mathrm{dy}+\mathrm{dz}$.

There are five possibilities for motion's emergence type constantly or partially by some intervals as $\mathrm{dx}=\mathrm{dy}=\mathrm{dz}$, $\mathrm{dx}=\mathrm{dy}, \mathrm{dx}=\mathrm{dz}$ and $\mathrm{dy}=\mathrm{dz}$ or none of them. $\mathrm{dx}=\mathrm{dy}=$ $\mathrm{dz}$ equation is not possible when it is checked over Eq. (207). For the others, if dx is taken from Eq. (207), and then if it is put on its place on mass conservation equation which is $\mathrm{ds}=\mathrm{dx}+\mathrm{dy}+\mathrm{dz}$, the equation becomes Eq. (207a).

$$
\mathrm{ds}=\mathrm{dy}+\mathrm{dz}-\frac{\mathrm{dy} \cdot \mathrm{dz}}{2(\mathrm{dy}+\mathrm{dz})}
$$

Here, assume that it is $\mathrm{ds}^{2}=\mathrm{dx}^{2}+\mathrm{dy}^{2}$ which is the projection of the same ds during forming a sphere by $\mathrm{ds}^{2}=$ $\mathrm{dy}^{2}+\mathrm{dz}^{2}$, where $\mathrm{dx}=\mathrm{dz}$ by the same angle vertically and horizontally according to a fixed reference; then Eq. (207a) becomes Eq. (207b).

$$
d x^{2}=d y \cdot d z+d z^{2}-\frac{d y^{2} \cdot d z^{2}}{4(d y+d z)^{2}}
$$

Over Eq. (207b), it can be said that $d x=d y$ and $d x=d z$ are not possible. For $\mathrm{dy}=\mathrm{dz}$ equation, it becomes $\mathrm{dx} / \mathrm{dy}=$ $33 / 16$; but if it is used on the main function Eq. (107), it seems that even it is not possible as well; therefore there is only one possibility left that none of them emerges even for any interval of motion, and the components are always different. They never intersect for any combination. Any point of free space has emergence priority due to the time differences that I already proved this by another method over geometry at the beginning of the paper. Even for after the comma which means in small amounts, between two different times, there must be an evaporation or in the other name vibration decrease, and it is recovered since there will be friction or collision by different magnitudes as non-flexible; so repeat and thus frequency are required to keep matter standing. A work is constantly done even in each small time. Matter is sum of many frames.

Here as you can see, $\mathrm{dx}, \mathrm{dy}, \mathrm{dz}$ are the smallest 3D derivative components of an unknown function whatever the function is; so as matter basically works over the same principle, namely if that components were for speed, distance, force or time by changing functions since the rule is going to be the same, for example if you knew $t$ as the same for any component during measuring distance over $\mathrm{x}=\mathrm{vt}$, then $\mathrm{v}$ would be different. If you knew $\mathrm{v}$, then $\mathrm{t}$ would be different. As also there is time differences, a motion is only able to emerge over arcs since there is no middle point for any force applied; so additionally if you know $t, \mathrm{v}$ becomes uncertain because of emerging irrationality; so in the exact opposite way of the wave function, even if you measure any physical value at different times even for different phenomenon, there is going to be no intersection point. You may get closer but equation. As a result, 3D position or for example 3D mass cannot be detected certainly if you know for example time. Even you cannot detect the other component if you know one of them. Namely, the uncertainty is not limited by momentum and destination. It is also valid for example between mass and destination or time and distance. As a result for like-this relations, you must relate them by both inequality and uncertainty like Heisenberg [2] did. Also you can write your own with coordinate system.

This is like the rule of circle. If you take the circumference as integer, then you make the radius uncertain as irrational. If you take the radius as integer, then the circumference becomes uncertain because of the relation between area and length of the circle.

Hence entropy also increases as long as there is motion. If you assume that there are infinite universes then the to- tal energy of the universe which is done to create it against space resistance must be provided by another universe or energy source. It means between two energy, there is an energy transfer. While our universe is emerging, the source loses energy, and when our universe lost, the energy stored again by the source; but as you can see this is not different then the energy transfer in our universe during creation or after creation. Namely, if there is a transfer, there is going to be vibration decrease and extra energy need. It means, the source is consumed by $\mathrm{mc}^{2}$ each second. It means our universe consuming many universes, as the other universes consume many other that also source universes need energy from other one, then where is the extra energy is provided from. Already our universe also must be source of another one.

If you think there are limited universes that the number can be like $10^{10^{10000}}$, as infinity can never work since anything is element of it namely there is no energy change in infinite energy, these universes would spend $\mathrm{mc}^{2}$ even in infinite sum. Namely energy spent by these universes for 1 second is equal to infinite period of their life time spending. As a result there cannot exist infinite universes at the same time with infinite transfer. Infinite transfer amount means emergence from nothingness constantly each second since requires infinite energy decrease to 0 and then increase from 0 . This is non-sense. Already it cannot be over time. It happens untimely manner and first time creation is not possible.

Already as you can see, there is no constant speed due to the components. It also means there is acceleration or deceleration. Acceleration is only possible from zero point that is not possible; but deceleration is possible from infinite, absolute energy, and emerging things are its virtual parts with frequency. There is an imaginary time point that I proved it mathematically at the beginning of the paper. There are imaginary time, motion and energy which are not real.

\subsection{Enuma Elish Tablets: The Babylonian Epic of Cre- ation}

Because of the deceleration from an infinite value by forcing each second, there can never be an emptiness in the other name absolute energy absence as matter is created over God or infinite space;so as far as we can see in holy books

"The spirit of God was hovering over the waters, and God said that let there be light, and there was light. God saw that the light was good, and He separated the light from the darkness."

\section{Genesis: The Beginning}

and

"He is he who created for you all things that are on the earth, after that as he established the throne on the sky he constructed it as seven skies."

Koran 2:29

and

"He sits enthroned the above the circle of the earth, and its people are like grasshoppers. He stretches out the heavens like a canopy, and spreads them out like a tent to live in."

Isaiah 40:22

and

"God reigns over the nations; God is seated on his holy throne."

Psalm 47:8 
"Did not the unbelievers see that when the sky and the earth were close, we uncoupled them, and created all living things from water."

Koran 21:30

verses talk about water in the other name space or sky or heaven; but the creation from water of living things should aim drinking water.

Also, as it was said in this article or in the other one [1], the universe emerges as a result of centrifugal force. During expansion, the waves inter-wined and formed the particles.

\begin{abstract}
"When the heavens above did not exist; and earth beneath had not come into being; there was Apsû, the first in order, their begetter; and demiurge Tia-mat, who gave birth to them all; they had mingled their waters together."
\end{abstract}

\section{Tablet 1 of Enuma Elish: 1-5}

part also can be assumed as an evidence for this.

Some strong information maybe have been damaged in time; but I think, there were many prophets as actually these information are heavenly information as a prophet doctrine, that you can see them in Quran, Bible or Torah, as also I stated them at the above and in the article I published [1].

"There never was a people, without a warner having lived among them."

Koran 35:24

verse may be an evidence for it.

\subsection{Is Nibiru Vega Star?}

As visible light loss again, as I said [1], the black hole radius of $\mathrm{r}=\sqrt{\mathrm{mG} / \mathrm{c}}$ where the gravitational acceleration is equal to the magnitude of the light speed for 1 second since gravity is like constant speed movement, any frequency photon loses its energy at the end of 1 second in this field. Already as it was said, Schwarzschild radius black hole stops its own vibration because of the superposition of each particle it has. Vibration of the particles in Schwarzschild radius black hole firstly increases extremely, that causes vibration stop at the end of 1 second as caused a denser space which does not allow particle creator wave to form particle over space. In the same manner black holes which have $\mathrm{r}=\sqrt{\mathrm{mG} / \mathrm{c}}$ radius, have smaller mass and bigger volume than Schwarzschild radius black hole will also evaporate; but it will be slower than Schwarzschild radius black hole.

For these information, it may be said, that maybe Vega Star lost its black hole force by slowly opening itself because of the frictional evaporation.

As a result, If a mass gets denser, it always becomes darker, and finally becomes a black hole if the density increases enough.

“...Until when he reached the setting place of the sun, he found it setting in a pool of murky water.. ..Until he reached the rising place of the sun, he found it rising on a people for whom we had made no shelter against it..."

\section{Koran 18:83-99}

verses about Dhu'l Qarnain may be evidences for this, and also Gog and Magog who are told in the whole verses may be aliens. Here, the shelter can be atmosphere of a planet.

Our sun and its system are going to Vega as we know it on Solar Apex which is the path of our sun system; so maybe "setting place of the sun" is Vega in this verse. Also Nibiru has a meaning like "mass got the center"; so maybe they gave this name for these reasons as it has some different but perfect meanings which render its different properties. Also already Nibiru is known as transition planet because of its name. Maybe this name was used as transformation transition from black hole to a visible star. Also as Vega star was North Star before, it may tell its relative transition besides our sun system orbital.

" Nibiru, which is said to have occupied the passageways of heaven and earth, because everyone above and below asks Nibiru if they cannot find the passage. Nibiru is Marduk's star which the gods in heaven caused to be visible. Nibiru stands as a post at the turning point. The others say of Nibiru the post: "The one who crosses the middle of the sea (Tiamat) without calm, may his name be Nibiru, for he takes up the center of it." The path of the stars of the sky should be kept unchanged."

\section{The Myth of a Sumerian 12th Planet}

may be an evidence; because as you can see, there is a sentence, that is "the gods in heaven caused to be visible." It may be an appearance from black hole. Also the passage may be worm holes which also we look for them. It is very sensible if a bright star suddenly occurs; because you think that there is door. Also there is a verse in Quran like

"I swear to the sky and Tariq. What informed you about Tariq? It is the star of piercing darkness."

\section{Koran 86:1-3}

and so also it may also be an evidence for Vega Star. Already Tariq means "pound" and "suppressed".

Actually, they are not only reasons which create doubt on me. Namely,

- You do not need such a small radius like Schwarzschild radius. Even many times bigger radius can create a black hole by $\mathrm{r}=\sqrt{\mathrm{mG} / \mathrm{c}}$.

- Vega has very high spin even for such a diameter. Namely, if you compress it to a lower volume, it is going to spin faster as required by conservation of angular momentum. Maybe was spinning before like this.

- There is no space stuff around it. Maybe It ate or distributed them.

- Everything is together with a probability. You can count something as anomaly since is not usual; but maybe there may be some unknown effects which stop nuclear reaction even if also is going to start again due to foreign matter, spin, volume, heat and density change.

\section{Adaptations}

\subsection{Energy generating}

\subsubsection{Basic infrastructure}

As it was said the above, it does not differ for matter, that it is created or it does work. Matter always does work even if it does work relatively to us in outer space observational area by interacting another space elements by using its own energy without external energy source as a head worker or when made it worked by man-made forcing with external energy being the matter is sub-worker which means connected to a system. To be more valuable of a vehicle or device does not mean for matter it is valuable. Matter is doing the same work for any material and work even if it is static or is moving. At that time, it is only emerging.

Matter is a part of infinite. You can neither detract anything from, nor add anything to infinite, since everything an 
element of the infinite; so matter is created by forcing each second as an absolute must do the work instead of the created one, and is created by a repeated motion. This means that matter is absolute inert. This means $\mathrm{mv}^{2}(\mathrm{Js})$ energy potential for a formation velocity of $\mathrm{v}$. As formation will be in changing values during 1 second, energy must be shown as (Js) which means "Joule per second", and also mass must be shown as (Kgs) which means "Kilograms per second". These things mean that we can use matter's own energy each second by using matter itself without spending it and external energy resources. As it always does a formation motion, still it will be doing a formation motion.

$$
\varepsilon=-\frac{\partial \Phi_{\mathrm{B}}}{\partial \mathrm{t}}
$$

Eq. (208) is Faraday's law of induction, and it states that changing magnetic field produces an electromotive force (emf) in Voltage unite by creating a potential difference and an electric field.

Handle a simple machine system which provides force efficiency and generate electricity by a lever by $\mathrm{Ld}_{1}=\mathrm{Fd}_{2}$ where $\mathrm{L}$ is load, $\mathrm{d}_{1}$ the distance of the load from the center of gravity, $d_{2}$ is the distance of the force applied from the center of gravity. For the load which requires E energy to be lifted, the equation becomes Eq. (209),

$$
\mathrm{Fx}=\mathrm{E}
$$

where $\mathrm{x}$ is the distance taken while applying the fixed magnitude force. If the used magnetic flux is increased by making magnets bigger gradually forever for the fixed force efficiency distance of $\mathrm{d}_{2}$ which is the other hand of the system, even if the magnetic flux change number is the same, it becomes Eq. (210) as the distance taken of $x$ must decrease to produce the same E energy for the same magnitude force.

$$
\lim _{\varepsilon \rightarrow \infty} x=0
$$

It also requires to be 0 of the work done. The distance taken for each change must decrease at the amount of the enlarged magnets for the same force to produce the same amount of electricity; thus the work done decreases even if the generated energy is the same or increases. Finally even electricity can be generated without external energy, that means zero external work done if it is used big enough magnets. In a high magnetic field, self-inflicted electron flow which means electricity can be detected beyond the same order of spins because of electrons' movement which occurs at different distances around their atom. Even if the high violence magnetic field is fixed without change over time, the movement of the electron which does not jump another atom in this magnetic field around its atom can be counted as magnetic flux change. This work is done matter's own energy as matter is always together with a motion. This is like the condition of a gearwheel spinning at a fixed speed. If you give a handle to the system, the gear will hit the handle forever and will work, and the gear's energy is in God's hands. We can force matter by some incitements to work in the name of us by using some amounts of its own $\mathrm{mv}^{2}(\mathrm{Js})$ energy for a formation speed of $\mathrm{v}$.

For example in a simple machine system which provides force efficiency, if you enlarge the magnets gradually for the fixed force efficiency distance which is the other hand of the system, even if the magnetic flux change number is the same, the taken distance for each change must be decrease at the amount of the enlarged magnets for the same force; thus the work done decreases even if the generated energy is the same or increases. For example we can use instead of the lever two generators/motors to generate electricity. Let us assume, that one of them is $5 \mathrm{kw}$ at $100 \mathrm{rpm}$, and the other one is $1 \mathrm{kw}$ at
$500 \mathrm{rpm}$. If you run the $1 \mathrm{kw}$ with an external electricity and then use $5 \mathrm{x}$ radius gear on the $5 \mathrm{kw}$ for $1 \mathrm{kw}$, you can turn the $5 \mathrm{kw}$. After that the $5 \mathrm{kw}$ can feed the $1 \mathrm{kw}$ by $1 / 5$ of its energy. Only the magnet's weight effects the condition. Self-feeding systems are possible with or without magnets by many different techniques also with some auto vacuum layers against sound.

Also $\mathrm{d}_{2}$ can be lengthened to catch the same effect if magnetic flux increases; because when $\mathrm{d}_{2}$ is lengthened the force magnitude will increase and the distance taken will decrease automatically.

But here do not make a calculation like in classic mechanics. For example, if you accelerate a magnet on a rail by $+\mathrm{Ft}$ work, by required assembly with $\mathrm{A}, \mathrm{N}, \mathrm{l}$ coil values and the other wire values, assume that at the entrance of the coils during its motion, two poles of the coil are connected to each other and so the induced current's magnetic field stopped the magnet by the same Ft as $-\mathrm{Ft}$. Here, the work done by you to accelerate the magnet is Fx but the work done by the coil is $\mathrm{Fx}+\mathrm{E}$ where $\mathrm{E}$ is stored energy for example in a capacitor; so you made magnetic field worked. Even if you do not use magnetic field of a magnet, it does not mean it does not work at that time. Also while you are using it, it does not mean it worked; so for example by a lever like this shape $\vdash$ that the intersection point of perpendicular two lines is center of gravity and is fixed from there to rotate of the system, assume that there is a magnet placed as SN at the top of the vertical line, and there is the same magnet bottom of the same line as well. While you are making the lever moved towards the bottom from the end of the horizontal line, the emerging opposite magnetic field from top will push the magnet and your force applied back but the other one which was placed at the bottom will help you as the pushing will be in the direction of your force applied when you use it as a electromagnet like a coil gun as the ratio of magnetic field and current as $\mathrm{B} / \mathrm{I}$ is independent of current. You can catch the same effect in a rotating field by two the same rotors; but one of them will be rotating in the opposite direction that it can be provided some simple gears, that actually even by a single rotor, it is possible as well. This can be realized even by a single magnet as well; because the calculation is not like done in the classic mechanics. Otherwise you make mistake and it will be hard to understand. Changing size of magnet and its magnetic field, distance, coil and wire options for the same amount of conductor will change everything.

Already if matter is able to be polarized, it can be said, that actually matter has a potential to keep up with. Namely, there are some upper and lower boundaries before formation changes by getting flexible. For example if you handle a point mass has $\mathrm{m}$ magnitude being two masses have the mass spinning around it at the same radius like $3 \mathrm{r}$, the sum of emerging force for a time and point is $\mathrm{F}=2 \mathrm{~m}^{2} \mathrm{G} / 9 \mathrm{r}^{2}$. After that if you change the radius like $2 \mathrm{r}$ and $4 \mathrm{r}$ for each of them by sliding them by $r$ in a way, you made them polarized. For these new values, the sum of the forces is $\mathrm{F}_{2}=5 \mathrm{~m}^{2} \mathrm{G} / 16 \mathrm{r}^{2}$. Namely the second condition has bigger energy. It means the mass decreases or it steals from medium, from total energy of the universe or both of them. It protect itself. If it is able to be polarized, also it is able to jump to another atom constantly. It can protect its own energy until a limit.

\section{Warning}

To be more clean of it, I think that I should explain it more detailed.

Here, $\mathrm{C}_{1}$ and $\mathrm{C}_{2}$ are coils that are connected to each other 


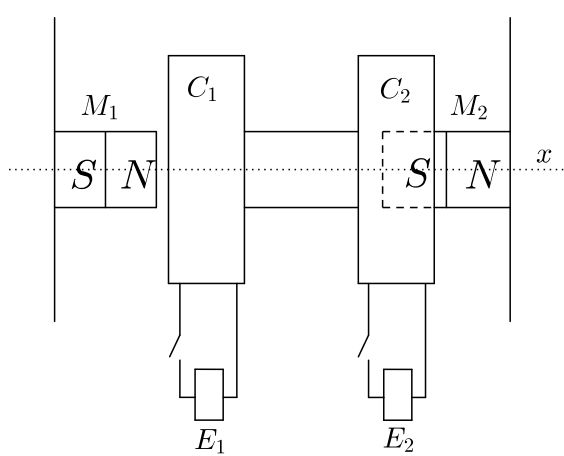

Fig. 40: Generator

by a material, and $\mathrm{M}_{1}$ and $\mathrm{M}_{2}$ are fixed permanent magnets on the walls. $\mathrm{E}_{1}$ is energy source like a battery that voltage and current are always fixed. As $\mathrm{E}_{2}$, it is a power storage unit. The coils are able to move right and left on a rail on $x$ axis. When both of the switches are closed at the same time, naturally first coil becomes an electromagnet and moves towards left namely the first magnet due to the direction of the current. During this time, the second coil is also moving in the same direction since both of the coils are connected to each other; but the second magnet will be inducing electricity on the second coil. Now, repeat the same action from the standard position by changing the first magnet's size and magnetic field forever gradually after switching off near the wall while moving the coils to the old and standard position. What happens there being the coil sizes and the current, the voltage from the energy source are fixed. The attraction between $\mathrm{M}_{1}$ and $\mathrm{C}_{1}$ will naturally increase for the condition because of the enlarged permanent magnet of $\mathrm{M}_{1}$; so $\mathrm{M}_{2}$ will be able to induce more electricity on $\mathrm{C}_{1}$ since change in magnetic flux relatively to time will increase. Here, the work done by $\mathrm{E}_{1}$ will always fixed since energy is fixed for $\mathrm{C}_{1}$. The work done by $\mathrm{M}_{1}$ will increase, and if you use bigger $\mathrm{C}_{2}$, also you can generate more electricity because of the attraction emerges between enlarged $\mathrm{M}_{1}$ and $\mathrm{C}_{1}$.

Also it is possible without magnets; because the ratio of magnetic field and current as B/I is independent of current; so many different size magnetic fields can be created by a current or induction can be realized for fixed magnetic flux change amount by changing properties of the coil and the same weight wire if you risk heat for more. Actually this can be used for thermoelectric generators to create heat difference.

Already because of the time differences, between each point of space that means emergence priority,

$$
\varepsilon=\mathrm{N}_{2}-\mathrm{N}_{1} \frac{\Phi_{2}-\Phi 1}{\mathrm{t}_{2}-\mathrm{t}_{1}}
$$

shows, that it is always $\varepsilon>0$ where $\varepsilon \in \mathbb{R}$. It means that a coil even placed at infinite distance from a magnetic field has a voltage difference between the two top of the wire. If there is a fixed matter in the core, voltage will increase. If there is a fixed magnet in the core, then even if also the coil is fixed, the voltage will greatly increase; so it means that even by switching two fixed coils have permanent magnet core and have different resistances will cause magnetic flux change; because electrons' orbital will slide even a current will not emerge, that it can emerge by required assembly and switching repeat. Maybe we can create a micro generator like producing a CPU that sheets between two permanent magnets will be connected serially to create high voltage difference to create current beyond sliding orbitals.
During generating free electricity, if you ask to matter like "What are you doing?", it says that "I am only emerging and doing a creation motion."; but at that time it is generating electricity relatively to you; so do not drain away the power of universe. Just use it.

The initial and the final energy values of matter can be different than the mean action; because each action is within a period of time as required by uncertainty, and matter performs an emergence action and goes through change in constantly changing environments and events; so a period of time is required for matter to place itself accurately in space. As these impacts emerge due to matter's own action, the state of matter can be turned into the state of performing action with lower energy, and continuation is possible. We can use matter's own motion of creation by using an incomparably energy, and we can force to make it worked for us.

Warning

\subsubsection{Additional examples}

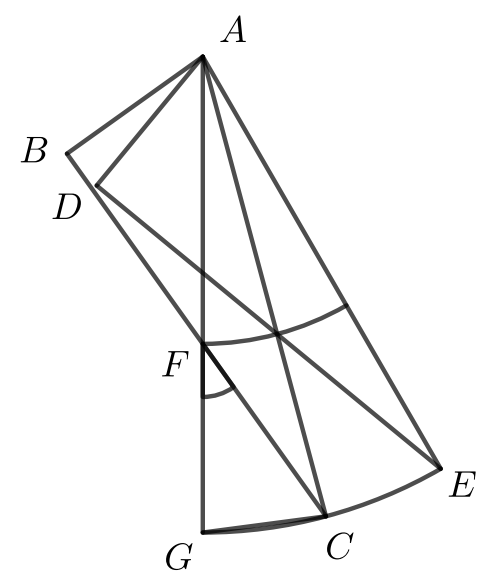

Fig. 41: Example of interaction between two dipoles. On the two circular objects rotating parallel to each other at the same center, there are dipoles positioned at different angles to attract or push each other.

In Fig. 41, there are two circular object sections of a complete circle, rotating parallel to each other with the center point $\mathrm{A}$. BC and DE to one of the circular objects; AG, AC and $\mathrm{AE}$ belong to the other and have gravitational power at every point of length. The GFC angle is the same for both butterflies and all other sections that complete the circle.

Under these conditions, $\mathrm{BC}$ and $\mathrm{DE}$ are the same poles and $\mathrm{AG}$ and $\mathrm{AC}$ are the opposite poles. In this approach, a reciprocal attraction will occur at each point along the lengths FG and FC within the FGC triangle and along the lengths FB and FA within the BFA triangle. The same applies to all non-shaped butterfly sections of the complete circular object. 
Since $\mathrm{F} / \mathrm{r}^{2}$ for each point will be directly proportional to the total gravitational fields, the total gravitance is 211 .

$$
\mathrm{F}_{\mathrm{BFA}}>\mathrm{F}_{\mathrm{GFC}}
$$

This means that the circular objects will move towards each other. In the course of the displacements that occur during the movement, the total attraction that occurs within each butterfly and between the butterflies adjacent to each other can always be allowed to move, for any destination of movement. This depends on the GFC angle and lengths.

This is a potential difference. The substance has motion and therefore an energy and mass, since a similar potential difference occurs. This makes it possible for producers who are completely mathematical and who produce their own energy on the basis of logic and save energy to the outside, and can work forever without the need for fuel or any external energy source.

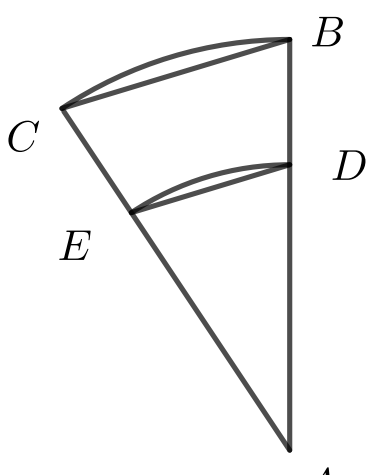

$A$

Fig. 42: Example of interaction between two dipoles. Opposite torque created by dipoles approaching each other on the same arm.

Here is another example in Fig. 42. AB and AC are onepiece lengths. Let $\mathrm{AB}=2 \mathrm{AD}$ and $\mathrm{AC}=2 \mathrm{AE}$. The interaction only occurs as $\mathrm{F}_{1}=\mathrm{F} / \mathrm{r}_{1}^{2}$ between points $\mathrm{E}$ and $\mathrm{D}$, and $\mathrm{F}_{2}=$ $\mathrm{F} / \mathrm{r}_{2}^{2}$ between points $\mathrm{C}$ and $\mathrm{B}$. $\mathrm{C}$ and $\mathrm{B}$ are the same, $\mathrm{E}$ and $\mathrm{D}$ are opposite poles. In this case, the torque inequality is $\mathrm{F} / \mathrm{r}_{1}^{2}>2 \mathrm{~F} / \mathrm{r}_{2}^{2}$ for $\mathrm{F} / \mathrm{r}_{1}^{2}>\mathrm{F} / \mathrm{r}_{2}^{2}$ force inequality, which is $r_{2}>r_{1}$ even when they are closest. If this is edited, 212 .

$$
\mathrm{r}_{2} \mathrm{r}_{1}>\sqrt{2}
$$

This means that the result can be compared to an irrational number, which means that even if one of the same lengths is fixed or both are moving, the motion will always continue even if it is shrinking. This is one of the possible results suggested by mathematics for irrational numbers, which, above, has always been said to be an irrationality due to its uncertainty. Cannot be static even for such an assembly.

Here is another example in Fig. 43. In the figure, there is a circular object rotating about point $\mathrm{D}$ and a small circular object connected to it by gears and rotating in the opposite direction as the large circle moves. Attraction or repulsion occurs only on BC length. For this condition, the potential

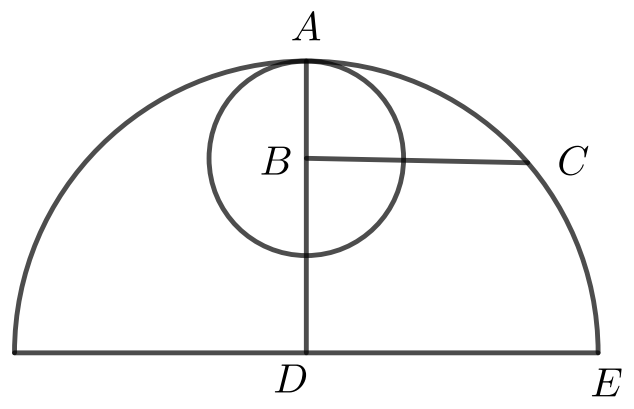

Fig. 43: Example of interaction between two dipoles. Opposite torque created by dipoles approaching each other on the same arm.

energy generated by the force generated in the large circle will be greater than the rotation effect of the small one, and there will be no rotation effect in the small circle at the time of the figure, causing movement and the circles start to rotate in the opposite direction. The gravity regenerates itself and movement each turn if the weight and friction of the entire system are large enough to include.

\subsection{Thrust source}

\subsubsection{Centrifugal force}

As producing thrust, as energy is not independent of force, it can turn into force. To realize this, Eq. (213) or if a blade is used instead of a marble placed at the top of a wire, nearly $\mathrm{F} / 2$ centrifugal force is perfect closed system thrust engine potential as independent of mass of air or water without opposite torque, and it can store huge amount of energy by a small energy amount when it is required by some transmission, that the work done is only done against frictions at that time.

$$
\mathrm{F}=\frac{\mathrm{mv}^{2} \sin (\alpha)}{\mathrm{r}}
$$

More thrust than jet fighters can be produced in the same volume without fuel and other external energy resources even by the electricity generating methods. Just use as double spinning in opposite directions by the same structure and propeller position if you do not want to use a butt propeller like in helicopters since a second rotation is going to emerge because of conservation of momentum.

In classical propeller systems, you must hold on air to produce thrust by propeller blades; so the mass for the energy of the work done is directly must be the vehicle which is aimed to move; but by the above stated method, you can only calculate the mass of a propeller but high speeds since the friction will get bigger. It can produce thrust as much as tensile strength of the used materials for the propeller and the blades. If it is connected around a rotating wheel, you can produce torque for any machine.

It is not possible to produce thrust by fixed blades. You must use movable blades which create different angle as long as rpm increased. By 
high enough voltage signals which carry enough current, some pulses make motors create this force by these blades. Right this point, in a limited area small motors are used numerously instead of 1 motor, and a phase difference is created. When one of them gets signal, then suddenly signal is cut, and the other one gets signal. First motor which gets first signal stops oneself or made it stopped by opposite current, for example when 10th motor gets signal, by order. This phase difference allows creating this huge force, and a loop is created. Already, since centrifugal force is dependent on speed by $\mathrm{v}^{2}$, not mass, lower mass of blades creates huge force, and small motors are used to gain more thrust in the same volume. It is already more suitable.

\section{WARNING}

Also you can use magnets. Due to aimed thrust, you can use attracting or repulsion between two magnets. By this way the propeller can turn freely like classic ones without a friction in the emergence way of the thrust.

\section{LSE offer}

I have an offer for light speed experiment.

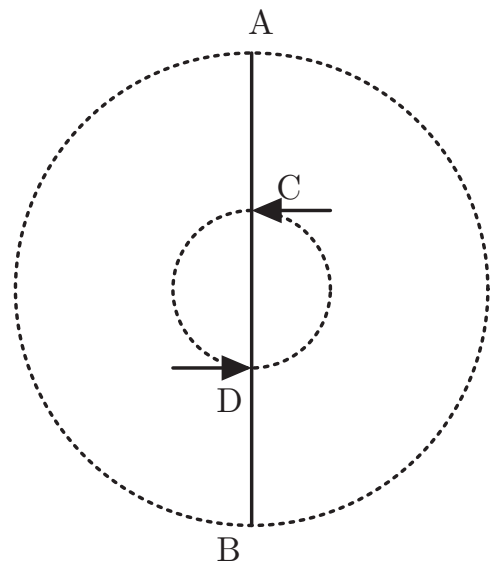

Fig. 44: An object which is about to go at light speed

Think, that the distance between A and B point is $\mathrm{c} / \pi$ where $c$ is light speed; thus if $\mathrm{AB}$ line spins each second 1 round, both A and B points go at speed of light; but for example $C$ and D point cannot go at that speed since they have not got the same linear speed; so for example if we want to detect how objects are seen at light speed or how length contraction and the other properties emerge, we can see what we want since we can protect our thrust source against physical condition negativeness near light speed; because we use the engines from for example $\mathrm{C}$ and $\mathrm{D}$ points to spin the line. There is going to be different behaviour along the $\mathrm{AB}$ line. During this, emerging centrifugal force is going to be just $\pi / 2$ times mass of the line.

This also can be used to collide huge blocks to produce new type of elements by fusion reaction.

\subsubsection{Imbalance}

To be one of them before or after of two the same opposite $\mathrm{Ft}$ work as $+\mathrm{Ft}$ and $-\mathrm{Ft}$ changes displacement. It becomes Eq. (214),

$$
-\mathrm{x}=\frac{\mathrm{Ft}^{2}}{-\mathrm{m}}+\frac{-\mathrm{Ft}^{2}}{\mathrm{~m}}
$$

where $\mathrm{x}_{\mathrm{a}}$ is the distance taken during acceleration, $\mathrm{x}_{\mathrm{d}}$ is the distance taken during deceleration, and $\mathrm{x}=\mathrm{x}_{\mathrm{a}}+\mathrm{x}_{\mathrm{d}}$.

In the same manner, if a particle is accelerated between two plates and then is decelerated between another two plates have more distance between the plates but the voltage is the same with the accelerating plates in an electric field, emerging resutant force as thrust becomes Eq. (215),

$$
F_{R}=m v \frac{t_{d}-t_{a}}{t_{d} t_{a}}
$$

where $t_{d}$ is the deceleration time and $t_{a}$ is the acceleration time. It does not have to be a particle acceleration. It is valid for any type of imbalance.

\subsubsection{Warp drive}

If you place two sheets on space that the distance between them is x relatively to your observation, and if you can collect the space between them around them, then you can see that an object which passes from between of them, passes faster and then again slows down and goes at the speed before it enters the area.

Actually this engine also can be counted as an imbalance engine, and it is possible to move objects at any speed. Namely it also can be used for slow space travels at usual speeds like classic ones. It is very good that it can be produced being there is no moving part. Even a solenoid coil can realize this. If you place a permanent magnet at the center of a solenoid coil which is used as electromagnet, it can produce thrust without particle need by changing orbital radius of the permanent magnet. Namely it holds on electrons without creating current. Think that you placed two different size magnets on a model car for attraction or repulsion. On this car also both magnets are on two smaller model cars have wheels, and then let them go. You will see that the main model car which carries both smaller car is going to move in a way. It cannot be static. This also can be realized by electromagnets in an air vehicle.

Also if you create a right assembly by electromagnetic fields, for example you can move each parts of the jumping ship by order at high frequency for faster than travels theoretically, the electromagnets themselves are included as well.

\subsection{Interstellar communication methods}

\subsubsection{Entropy and universal energy preservation}

The focal points of the universe which means particles, have been evaporating; but this evaporation cannot change the total mass and energy of the universe. Particles emerge over the space without having an external energy. As a zero resistance is required to be able to go at infinite speed, also particles cannot go at this speed; so particles lose energy and mass by evaporation as they have no external supporter energy. As particles emerge over a space which wants to be fixed as matter is created by a forcing each second, and as no work can be done untimely manner, a disorder period occurs and excludes particles in the other name space waves from the space which particles emerge over it. This is the same event with it, that if you stretch a curtain by hand which was already stretched, you feel a resistance. Like in this event, as particles have no other external energy, they lose their energies by some periods, even if external frictional and gravitational losses which are a second effect on each other are not included as well. Evaporation means decrease in vibration. Particles which are on a denser space tend to stop faster manner. Total energy and mass of the universe are fixed during this evaporation, and only its radius and density can change.

This feature of the universe can be used for communication. We can easily calculate the evaporation amount each 
second if we can achieve the identical simulation of the universe . This cannot be known theoretically as any formation will cause different density and so will cause different evaporation amounts. When we create a human made anomaly, we can compare the results, and can use these difference for instant communication.

If each effect is exactly at the light velocity, there is going to be always some extra property which causes faster than light transportation because of the way things work. Assume, that a rectangular space has 10 times squares which each of them holds $10 \mathrm{~Hz}$. Total rectangle emerges in 1 second; so the time difference between each square is $1 / 10$ second. When an object at 10 $\mathrm{Hz}$ emergence frequency and has the same volume with each square which is placed at one of them moves at the speed of 10 squares per second, this space can hold this object. If you want to move it by the same emergence frequency at the speed of 20 squares per second, the space naturally block you since there will no place to go yet since the movement path has not been emerged. If you use $1 \mathrm{~Hz}$ emergence frequency matter which has the same volume with each square namely each point of one square feeds its frequency thus existence, you can move it at the speed of 100 squares per second. If you think this for huge mass clusters in the universe, they may have a one-piece behaviour since actually already no mass can be counted as a single mass separately. Namely, some effects maybe already move at beyond speed of light as also we can use it as communication material. As we cannot detect faster than light things, we can only find it by simulations, by indirect calculations. Namely, the effect of the man-made anomaly is not always detected at the light speed by far places. Because of these reasons, I said in the Dark Energy section, that also space causes an acceleration even if it actually exactly is going to decelerate. We should calculate both acceleration and deceleration amounts of them together. Additionally, you must assume that the volume of the object has the same magnitude with that squares; because otherwise a second environment is going to occur with special properties. You must use the volume of the new object. This can easily cause special ports between matters for example between two magnetic pole. Some lines between them can let faster than light transportation while another one forbids. Also they can change over time. Namely, the same point will not let it more. We can develop communication ports by this way. Even spherical particles may stretch without losing its gravitational and pressure balance in a time interval since the act will be for protecting the current physical condition. It may take shape due to space. It is not an anomaly as also the same will be anomaly and will damage the particle when occurs out of required limits. It means, an electron may behave as a beam in a time interval during its transportation. We can make a cheap experiment by a huge solenoid coil. The center will be extremely lower density, and an electron can exist from the other side when it has just enter over light speed.

\subsubsection{Space collecting by small scale imitation of the starting condition of matter}

As it was said the above, matter uses the same space for any work; so the distance taken at the beginning of time was scanning of all the universe. This condition can be imitable. For example, if an electron creator wave scans more space for the same speed and if causes more mass density in an unit of volume, it can go faster than light even the light speed is fixed at that time but it is not formed by other sub-atomic particles; because as sub particles can behave differently for changing frequencies, also they can exclude extra drawn mass; so you should use the particles which are not formed by other subatomic particles, and you need a good vibration resource, that it was explained the below. During this transfer, naturally there will be a deformation amount.

Moving objects also draw more space; but this is only for protecting its total energy which is charged by an external worker. If you want to create an extra space to move of it faster than light at light speed, you must create 'overcharged' particles. In this way, also particles cannot go faster than light without external effect. If you find one of them one day as goes at higher speed than the light speed, it is worth the search. It means, there is an anomaly, that is possible.

\section{Warning}

As there is no absolute threshold value to be in accordance with uncertainty, relative extreme energies are able to realize this oneself without vibration sources; because by this way, forces emerge for each small periods of time will be taken over threshold value. For example, Magnetars may realize this. Maybe some of high energy particles are counted as still in the Magnetar which sent them even if they got place for example around the Earth at that time.

\section{Creating artificial sub-atomic, overcharged communication particles}

Formation of particles was during expansion of the universe by an ignored time differences between different particles. During expansion of the universe, as there were different density spaces, also for the fixed light speed and the basic mass particle, there were different size particles even the same basic formation particle always wanted to create the same particle, and they have different internal vacuum forces because of the emerging centrifugal force since different density spaces were emerging during the expansion because of change in the density of the universe. This does not mean that we need the same event with the same physical magnitudes to create a new particle. Many different frequencies to create different particles were emerging and decaying at the singularity of the beginning of time. These frequencies are like bicycle rim frequency that you suppose it turned back. It is possible to create new type of particles and atoms by using free space. We must pass many photon particles by an ignored time difference from the same point of the space, actually by using ionizer photons to gain the smallest time difference. We must create a big density. As each action is in a time interval, this event will start render a particle slowly or suddenly according to used particle number and energy. This is like digging water by stones. There will be a collapse and then suddenly will be an expansion. Photon will start to move around a circle by vacuuming the space. New type of elements do not have to be similar with the current ones. As they can have smaller number of subatomic particles, also even they can be formed as one particle nucleus and orbital particle, which do not consist of other subatomic particles. They can be heavier than the current ones millions of time, if the wave spins around required 
radius; but its life time will be short as the frequency and thus the friction frequency will be bigger. As we can build new planets have the same gravity with the planet earth, also we can create some particles which collect more space and can go faster than the light naturally. These ones can be used for communication as artificial subatomic communication particles.

\section{Vibration source}

When a particle passes through an electric field, during passing duration, the particle decreases the electric field's energy for other works; so if you assume that there is a fixed material in the magnetic field, the magnetic field would induce electricity during this passing on this material which is in the electric field as magnetic flux is going to change because of the energy sucking of the particle. As this method is pretty good vibration instrument to vibrate magnetic field by some particles in the magnetic field, also is a pretty good computer processor potential. We can align electrodes which are made of semiconductor or dielectric materials around a circle, and each one of them vibrates at visible light frequency. We can use noble gas nucleus or ion, or can use air particles directly from a magnetic plasma by rotating them in this circular area. It is possible to produce a coin size processor runs at $10^{20-25}$ Hertz. As this frequency is not because of a single particle, it is not going to be ionizer. To block constant acceleration of particles, we should only accelerate them by some periods. To use more particles or slow particles some time only effect the process number, and some usual fluctuation are not so important. Even its energy will not be as much as a toy laser.

To be smaller of data which is sent, will directly make energy and work done to send data smaller; so these processors must be used to process data before sending data. For example, we can record a data group like in the Table 3 by the most appropriate function or changing functions which are for remaining bits.

Table 3: The current data bits to be cut

\begin{tabular}{c|c|c|c|c|c|c|c|c|c|c} 
& 1 & 2 & 3 & 4 & 5 & 6 & 7 & 8 & 9 & 10 \\
\hline $\mathrm{A}$ & 0 & 1 & 0 & 0 & 1 & 1 & 0 & 1 & 1 & 0
\end{tabular}

This record would be according to $\mathrm{f}(\mathrm{x})=3 \mathrm{x}-2$ function for Table 3 data, and for example it is recorded as A124. This means that there are 4 pieces of 0 which the distance between each one of them is 2 . As this code is going to get bigger by increasing number of bits, the same will be done for the code itself again and again. It should be made as the smallest as possible by using new hardware or software characters, or by using uppercase and lowercase letters together with a required combination.

\subsubsection{Lowering mass for short distance communication}

As required by uncertainty, each work can only be done in a time interval. Also as it was said the above, being $t_{p}$ is the Planck time, the universe is spinning at $1 / t_{p}$ frequency for 1 second and it forms free space, after that it forms particles over the free space. It means that if particles are forced to be formed when the universe is not there, mass magnitude is going to decrease at the same amount. If emerging frequency of particles is taken to empty space by an empty space frequency, it means any mass can be transferred at near light speed or at high speeds.

\subsection{The Cryptobit}

In accordance with the uncertainty, any measurable physical value of matter increases or decreases between absoluteness and absolute absence but its loss. No physical value can take certain value. They can only be expressed as some approaches.

As a result of this condition, for example, if you want to measure process number of a CPU, you can only get some different numbers at least for the numbers after the comma each time of measurement. When ever you want to measure more sensitive, new different numbers emerge after the fixed numbers after the comma. Except this, you can never get a certain number. Additionally for example, if you make a division, for the same operation the processor always performs the operation in different times. Yes it performs by some packets like 32 or 64 and always gives to you the same number that actually this also is not exact, it does not perform at the same time for each repeat. If you set the interval to find out this and if you ask to it over a program, sometimes you can get some results like 33 or 65 by unpredictable changing frequencies like 1 of 50 or 20 for 1 second.

Actually this physical principle and condition can be used as a perfect unpredictable cyberspace security element; because it creates a way to select incalculable and unpredictable codes like the below.

\subsubsection{Selecting randomly}

Let us use rounding the decimal numbers into closest one. For example, if the number is like $\mathrm{x}, \mathrm{xxxx6}$ it is rounded into $\mathrm{x}, \mathrm{xxxxx}$ and this condition is called as 1 . If the number is like $\mathrm{x}, \mathrm{xxxx} 4$ then it is rounded into $\mathrm{x}, \mathrm{xxxx}$ and the condition is called as 0 . If the number is like $x, \operatorname{xxxx} 5$ try again. These 0 and 1 are not bits.

Also there is another way from many other ways that I used this second one. For example while a timer performs countdown, ask the time of counting until a number. If you repeat again and again the countdown, many times you get the same number but some times you get different number which is closest number to the actual number. The following VB codes are for this.

Just use 3 text boxes, 2 timers and 1 button, and then copy the code below. It counts from 1000 down to 998 by the interval of 1. Textbox3. Text is set as 3 by the interval of 10 . You can change them; but over these values, Textbox3. Text becomes 1 many times but sometimes becomes 2 if you repeat the same operation by the same button. You can use any frequency for the repeats over the same button. Especially use long interval clicks to be sure. You can also generate autorepeat program. You can download the following program called Single Counter.exe from the following address: SingleCounter

Note

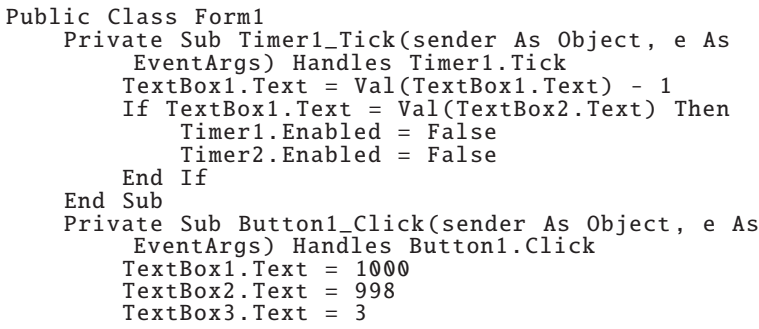




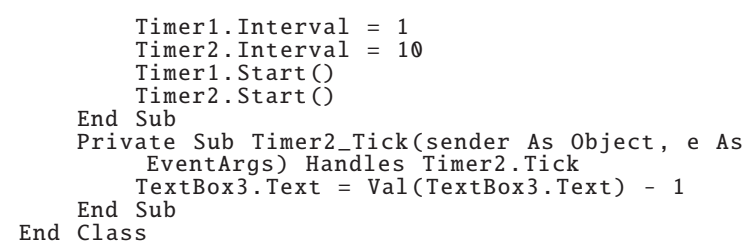

If you do not want to repeat more and thus not to wait, repeat the same event more at the same time like following program. If you increase the number of the counters, probability is going to increase.

This simple program shows us, that randomly changes the countdown time of each group. Sometimes one or more of them and some times the other ones become 1. You cannot guess. Just use 18 text boxes, 7 timers and 1 button, and then copy the code below. It counts from 1000 down to 997 by the interval of 1 over Timer1. Textbox3.Text is set as 3 by the interval of 10 . You can change them; but over these values, Textbox3.Text and the other textboxes has numbers $6,9,12,15$ and 18 become 0 many times but sometimes become 1 if you repeat the same operation by the same button. Also they do not get the same numbers together at the same time. You can use any frequency for the repeats over the same button. Especially use long interval clicks to be sure. You can also generate auto-repeat program. If you do not want to wait and want certain results, you can increase the number of textboxes by the same rule. You can download the following program called Large Counter.exe from the following address: LargeCounter

Note

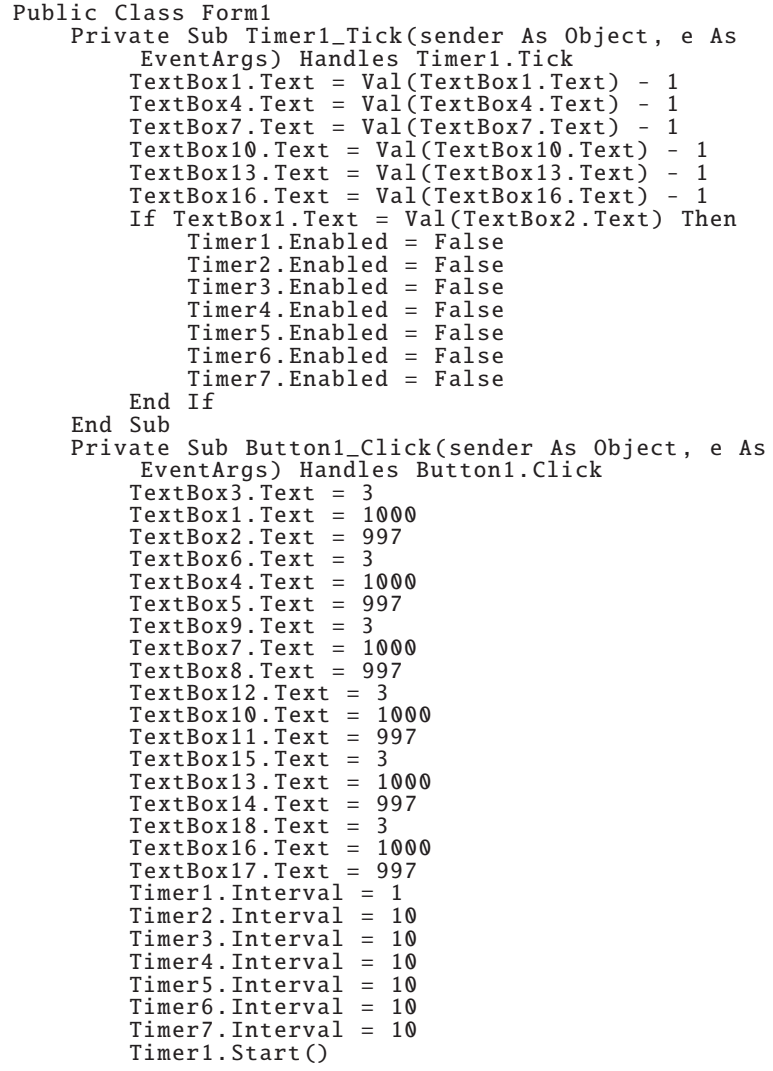

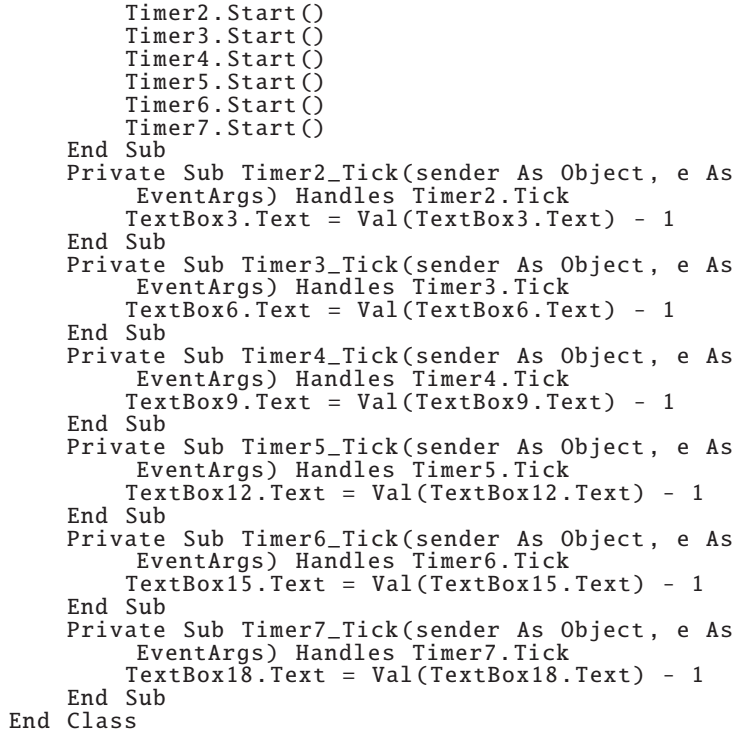

\subsubsection{Encoding}

Let us use the second method. Now assume, that English Alphabet is used for the key. 13 of the letters are going to be selected for using instead of 1 and the other 13 of them are going to be used for 0 that these are 0 and 1 of the bits. First of all for example let us choose the letters for 1. For this I wrote a simple program over MS Visual Studio Community as the above. You can write your own timer by using own language and own software or hardware architecture or you can use another language. These would be very sensitive and thus you can get better results. This is just for example.

Now assume that there are 26 pieces of the timers for each letter. Click on the button or repeat the action by an automated program until at least 1 of them becomes 1 over the Large Timer. If more than 1 of them become 1, then you are going to repeat only for the different group until only 1 of them becomes 1 . Finally you selected 1 letter for encoding bit 1 . Repeat the same for the other 25 letters until there exist only 16 pieces. After that the rest namely the existent other 16 letters are going to be used for encoding bit 0 .

\section{Offline Calibration}

While determining the key for bit 1 and bit 0 over the letters, two computers which are aimed to be connected to each other for communication, the first key is recorded for both of them at the same time, and then they become online. Even the system architects which are built the machine cannot know the key. Now assume that each communication parts are performed by some certain bit lengths. Namely two computer always send $10 \mathrm{~KB}$ data parts even if the data is shorter then this. After each $10 \mathrm{~KB}$ packet, the key changes. The changer computer sends the new key; but this new key is also determined for the last time over the previous key. This previous key was already random namely even the system architects do not know. The second computer receives the new key and open it according to the previous key. This repeats itself continual manner in this way. Namely nobody knows what the two computers do. We made them confidants. Even attacks can disrupt communication and packets but also they cannot steal from us even if we cannot perform communication. Nobody is going to know what the key is going to be next time approximately. It is not the solution recording only the calibration. You must save any act always. If you are a thief or a wicked engineer, then if you lost only one $10 \mathrm{~KB}$ packet for 
the life time of the communication during recording from the beginning, you cannot do anything.

\section{Remote Calibration}

This may be dangerous if you think that any signal is recorded by a third unknown computer. For the worst possibility, think that a user is recording any act and making a simulation of the communication by some algorithms over any possibility even by using new hardware architecture to catch the copy of the computers and the software. Namely making them sensible.

Actually the solution is making the data packets smaller and thus making the key change at a high frequency at last only during remote calibration.

\subsubsection{Conclusion}

As it can bee seen, Any information and the information searched for become the same after a point relatively to us. If you have only one bit for encoding like 1 or 0 , it can get any letter. Namely there are 26 possibilities for 1 or 0 . If you have a bit group like 10,11, 01 or 00 each one of them can get the same letters. You cannot decide which one of them is the main aim if you do not know the random key. The condition is the same for any length bit group. Do not forget that a second selection is done between each 16 letters for 1 and 0 . Namely for each 1 in a bit group, 1 of 16 letters will be selected randomly. Namely according to a hacker, 1 or 0 can get any letter. The stolen information can mean anything.

The key is also determined randomly. The builder system architect people also cannot know what is going to be the key. An approximately guess is also not going to be possible. It can suddenly be the worst and unpredictable possibility. Namely does not give benefit to kidnap and threaten the engineers for the key.

To work of this build in the best manner, actually a new hardware support is required. Especially for some specific communication instruments, you should build a new hardware architecture, and then the hardware will be doing the selection over unknown new hardware characters or frequencies. Even so, it can block most of the disturbing attacks by some simple programs. For example a simple windows emulator can block this. All windows is going to work over a simple encoding program first. Even computers are going to answer to a key-logger software or email by meaningless characters. Namely actually they can steal from us but cannot know what that information mean. It can be any information.

\section{Mathematics}

I published some of them before; but I noticed that there are some serious logic voids to explain the main subjects; so as I made some corrections and changing for some of them, also I added some new information and findings.

\subsection{Prime numbers}

\subsubsection{Multiplicity of prime numbers, and Floor function}

All prime numbers except 2 are odd whole number which have no positive divisors other than 1 and itself.

\section{Argument 1}

All of odd numbers which are not prime numbers are numbers which have at least two multipliers whether are primes or not, and multiplication in a way dependent of $\mathrm{f}(\mathrm{x}, \mathrm{y})=$ $(2 \mathrm{x}+1)(2 \mathrm{y}+1)$ function whose variants are within the range of $[1, \infty)$. If it is required to organize and demonstrate it in a table, the table would be Table 4 .
Table 4: Non-prime odd numbers

\begin{tabular}{c|c|c|c|c|}
$\mathrm{f}(\mathrm{n})$ & $6 \mathrm{n}+3$ & $10 \mathrm{n}+5$ & $14 \mathrm{n}+7$ & $18 \mathrm{n}+9$ \\
\hline $6 \mathrm{n}+3$ & $3 \times 3$ & $5 \times 3$ & $7 \times 3$ & $9 \times 3$ \\
\hline $10 \mathrm{n}+5$ & $3 \times 5$ & $5 \times 5$ & $7 \times 5$ & $9 \times 5$ \\
\hline $14 n+7$ & $3 \times 7$ & $5 \times 7$ & $7 \times 7$ & $9 \times 7$ \\
\hline $18 n+9$ & $3 \times 9$ & $5 \times 9$ & $7 \times 9$ & $9 \times 9$
\end{tabular}

There are the same rules vertically and horizontally. Each number in the table least has 1 odd whole number divisor; so the odd whole numbers which are not included in here, are only prime numbers.

If prime numbers were finite, over Eq. (216),

$$
\sum_{x=1}^{\infty} \sum_{y=1}^{\infty}\left(\frac{2 x+1}{(2 x+1)(2 y+1)}-1\right)
$$

the result of the total operation of Eq. (216a)

$$
\sum_{y=1}^{\infty}-\frac{2 y}{2 y+1}
$$

would equal to 0 for each y value, where assuming $2 \mathrm{x}+1=$ $(2 \mathrm{x}+1)(2 \mathrm{y}+1)$ and $(2 \mathrm{x}+1)(2 \mathrm{y}+1)$ are non-prime odd numbers; however this operation never converges, and each result is different; so it can be maintained as

Prime numbers are infinitely many numbers together with the odd numbers which can be divided by another odd number.

\section{Proposition 1}

Now that, prime numbers are infinitely many numbers; so number of prime numbers for any range determined, which odd whole number is the prime number has $\mathrm{n}$ order number, prime factors of any number determined and its multipliers or number of prime factors, and which odd whole numbers these multipliers are, can be found out.

Argument 2

All positive whole numbers are between $(2 \mathrm{n}+1)^{2}$ and $(2 \mathrm{n}+3)^{2}$ according to Table 4 ; because if a number is given and finding out which column is the last column it exists is waited for, as columns change by this rule, also it can be said, that Table 4 starts from perfect square belt. This belt is a reference for elimination; so for $b=[1, \infty)$, perfect square of each $\mathrm{a}=2 \mathrm{~b}-1$ positive odd whole number is $\mathrm{a}^{2}=1+4 \mathrm{~b}(\mathrm{~b}-1)$. To find out given number is which number's perfect square for floor value of the square root of the given number, it is required to create a function which rounds down any fractional part to whole value. For any x/y operation based on the simple division rule of $\mathrm{yn}+\mathrm{m}=\mathrm{x}$ where $\mathrm{r}=\mathrm{x}-\mathrm{yn}-\mathrm{m}$, Eq. (217) can be used.

$$
\lfloor x / y\rfloor=\sum_{n=1}^{\infty}\left(\sum_{m=0}^{\infty} \frac{1-(-1)^{2^{r^{2}}}}{2}\right)
$$

where $\mathrm{v}$ is the given number, and $\mathrm{r}=\mathrm{v}-1-4 \mathrm{n}(\mathrm{n}-1)-\mathrm{m}$, and also the upper limit can be $(x-y)^{2}$ instead of infinite. The 
result is a $\mathrm{s}$ number, and it provides the maximum number of columns which will be studied for the number determined. Here, result of $2 \mathrm{~s}+1$ operation is the basic odd number which forms the last column.

When each $2 \mathrm{n}+1$ number is used to divide the given number $\mathrm{v}$ for $\mathrm{n}=[1, \mathrm{~s}]$ interval, where $\mathrm{s}$ is the result of the perfect square elimination over Eq. (217), it is enough to check v is prime or not; so to check a positive odd number is prime or not, $\mathrm{s}$ pieces operation will be enough.

\section{Argument 2.1}

If both of the first multipliers of the given number were in the last column, where $\mathrm{s}$ is total column number or order number, then the given number only would able to be $(2 \mathrm{~s}+$ $1)^{2}$. This condition is only possible, if number determined is a perfect square. If the first two multipliers were in any column or columns after the last column, then the smallest number which can be emerge would be $(2 \mathrm{~s}+2)^{2}$. This is a bigger number than the number determined itself; so at least one of the multipliers are only in the column number s or in the columns before the column number s.

\subsubsection{Primality test for input number}

As it is not suitable to check whether an even number is prime or not, a mathematical interpretation can be done to check $\mathrm{v}$ positive odd whole number is prime or not. When it becomes $\mathrm{d}=0$ for $\mathrm{d}=\mathrm{v}^{-}\left((2 \mathrm{a}+1)\left(\left\lfloor\frac{\mathrm{v}}{2 \mathrm{a}+1}\right\rfloor\right)\right)$ whole value element, $2 \mathrm{a}+1$ positive odd whole number is aliquot divisor of $\mathrm{v}$ positive odd whole number; so $\mathrm{v}$ is not prime. In other conditions, it is not prime, and when $2 \mathrm{a}+1$ divisor is tested within $\mathrm{a}=[1, \mathrm{~s}]$ range, $\mathrm{v}$ is prime if the status of $\mathrm{d} \geq 1$ is conserved; so Eq. (218)

$$
f(v)=\sum_{a=1}^{s} \frac{v\left(1+(-1)^{2^{d}}\right)}{2}
$$

operation provides $\mathrm{v}$ number if $\mathrm{v}$ number is prime. Otherwise the result is 0 .

\subsubsection{Number of prime numbers in a range}

Number of prime numbers between $[2, \mathrm{v}]$ interval can be found out over Eq. (219),

$$
2+\sum_{\mathrm{x}=2}^{(\mathrm{v}-1) / 2} \frac{1+(-1)^{2^{\mathrm{d}}}}{2}
$$

where $\mathrm{v}=2 \mathrm{x}+1$ and $\mathrm{d}=\sum_{\mathrm{a}=1}^{\mathrm{x}-1}\left(2 \mathrm{x}+1-\left((2 \mathrm{a}+1)\left(\left\lfloor\frac{\mathrm{v}}{2 \mathrm{a}+1}\right\rfloor\right)\right)\right)$.

If it becomes $\mathrm{d}=0$ for an a value, $2 \mathrm{x}+1$ is not prime. Otherwise, it is assumed that is yet prime. Eq. (219) can be transformed for number of prime numbers within any range as well.

\subsubsection{Prime number has n order number}

For prime number which has n order number, within any range,

$$
\mathrm{p}=\sum_{\mathrm{y}=2}^{\infty} \frac{\mathrm{y}\left(1-(-1)^{2^{\mathrm{m}^{2}}}\right)}{2}
$$

Eq. (220) operation provides $\mathrm{p}$ which is the prime number has $n$ order number over Eq. (220a), where Eq. (220a) is based over Eq. (220), and $\mathrm{d}$ is $\mathrm{d}=\sum_{\mathrm{a}=1}^{\mathrm{x}-1}(2 \mathrm{x}+1-((2 \mathrm{a}+$ 1) $\left.\left.\left(\left\lfloor\frac{\mathrm{v}}{2 \mathrm{a}+1}\right\rfloor\right)\right)\right)$

$$
\mathrm{m}=\mathrm{n}-2-\sum_{\mathrm{x}=2}^{\mathrm{y}} \frac{1+(-1)^{2^{\mathrm{d}}}}{2}
$$

\subsubsection{Number of prime factors}

The odd numbers which are tried until the main number of the last column will not be enough for the operation of finding prime factor; because this operation is necessary for finding out one of the prime factors, and the other multiplier can be exist after the last column; therefore, to find out number of prime factor, all exponents of the main numbers which form the columns must be divided by the number determined in a way it does not exceed the number determined, and checked again.

$\mathrm{d}$ operation for full value of $\mathrm{v} /(2 \mathrm{a}+1)^{\mathrm{n}}$ must be $\mathrm{d}=$ $\mathrm{v}-\left((2 \mathrm{a}+1)^{\mathrm{n}}\left(\left\lfloor\frac{\mathrm{v}}{(2 \mathrm{a}+1)^{\mathrm{n}}}\right\rfloor\right)\right)$ over the greatest integer function of Eq. (217); so Eq. (221) calculates how many exponents of $2 \mathrm{a}+1$ numbers for each a value are aliquot divisors of $\mathrm{v}$ for each number determined.

$$
\mathrm{t}=\mathrm{v}-\frac{\mathrm{v}}{(2 \mathrm{a}+1)^{\sum_{\mathrm{n}=1}^{\infty} \frac{1-(-1)^{2 \mathrm{~d}}}{2}}}
$$

After that, when $\mathrm{v}$ is divided, left is prime factor itself, and when it is subtracted from $\mathrm{v}$ again, if the result is equal to 0 , then $2 \mathrm{a}+1$ number is not a multiplier. If the result is bigger than 0 , then it is a prime factor;

$$
f(v)=\sum_{a=1}^{s} \frac{1+(-1)^{2^{t}}}{2}
$$

so, Eq. (222) provides number of prime factor for $\mathrm{v}$ which is the positive odd whole number determined. If the result is still $0, \mathrm{v}$ is a prime number.

\subsubsection{Appendix}

\section{Number of prime numbers up to input number}

An operator like Eq. (223) where $\mathrm{s}=\frac{\mathrm{v}-9}{6}+1,9$ is the first number and 6 is the amount of increase, shall give $s$ which is the total column number for the input number of $\mathrm{v}$, when the result is rounded down for the numbers after the comma if the result is decimal. It is rounded down; because otherwise the next column will be included.

$$
\mathrm{s}=\left\lfloor\frac{\mathrm{v}-3}{6}\right\rfloor
$$

When column number is determined, after that number of non-prime numbers is determined up to the last column $\mathrm{s}$ or the total column number of s. After that there will be a final operation which is to determine and subtract mutual ones' number from number of non-prime numbers.

As the table's rule is $f(x, y)=(2 x+1)(2 y+1)$ where $\mathrm{x}, \mathrm{y} \in \mathbb{Z}$ and $\mathrm{x}, \mathrm{y}>0$, for an input number $\mathrm{v}$, number of non-prime number up to the last column the last column is 
included as well is Eq. (224) or Eq. (224a),

$$
\begin{gathered}
\sum_{\mathrm{x}=1}^{\mathrm{s}}\left\lfloor\frac{\mathrm{v}}{4 \mathrm{x}+2}\right\rfloor-\frac{1}{2} \\
\left\lfloor-\frac{\mathrm{s}}{2}\right\rfloor+\sum_{\mathrm{x}=1}^{\mathrm{s}}\left\lfloor\frac{\mathrm{v}}{4 \mathrm{x}+2}\right\rfloor
\end{gathered}
$$

where $\sum_{\mathrm{x}=1}^{\mathrm{s}}\left\lfloor\frac{\mathrm{v}-2 \mathrm{x}-1}{4 \mathrm{x}+2}\right\rfloor$ over Eq. (223).

Now, the mutual ones are subtracted from Eq. (224) or Eq. (224a). As each $2 a+1$ number for $a:[1, s]$ interval will give main number of each column which has a order number, we will subtract from 3 each multipes of the numbers from 5 to $\mathrm{s}$; from 5 , each multiples of the numbers from 7 to $\mathrm{s}$ etc. For example for 3 which is the main number of the first column, we must know how many multiples of 3 exist in the frist column. To find out this we must use Eq. (224) up to $\mathrm{s}=1$, and let naming this multiple number as $\mathrm{x}_{1}$. After that the first colum's separation rule as $6 \mathrm{x}_{1}+3$ is equalized to separation rule of 5 as $10 \mathrm{x}_{2}+5$, to 7 as $14 \mathrm{x}_{2}+7$ and to 9 , as $18 \mathrm{x}_{2}+9$ etc. Hence, the rule turns into Eq. (225),

$$
\sum_{n=1}^{s}\left(\sum_{x=1}^{s-n+1} x_{2}\right)
$$

where

$$
\begin{aligned}
& \mathrm{x}_{2}=\left\lfloor\frac{(2 \mathrm{n}+1) \mathrm{x}_{1}-\mathrm{x}}{2 \mathrm{x}+2 \mathrm{n}+1}\right\rfloor \\
& \mathrm{x}_{1}=\left\lfloor\frac{\mathrm{v}-(2 \mathrm{n}+1)}{4 \mathrm{n}+2}\right\rfloor
\end{aligned}
$$

Hence, number of prime number becomes Eq. (226) for an input number of $\mathrm{v}$ if $2,3,5$ and 7 are included as well.

$$
\mathrm{p}=4+\left\lfloor-\frac{\mathrm{s}}{2}\right\rfloor+\sum_{\mathrm{x}=1}^{\mathrm{s}}\left\lfloor\frac{\mathrm{v}}{4 \mathrm{x}+2}\right\rfloor-\sum_{\mathrm{n}=1}^{\mathrm{s}}\left(\sum_{\mathrm{x}=1}^{\mathrm{s}-\mathrm{n}+1} \mathrm{x}_{2}\right)
$$

Here, if it is certainly known that the input number is a prime number, then as the input prime shall directly become the last prime for number of total prime number, also order number of the input prime in prime numbers shall be found out directly by this way.

If the prime number has $m$ order number is required, $p$ operation is repeated up to $\mathrm{m}-\mathrm{p}$ becomes 0 ; so repeat number shall directly be equal to order number of $\mathrm{m}$. To realize this, an operator like Eq. (227) can be used as also it was used before for the above stated prime functions.

$$
\mathrm{p}_{\mathrm{m}}=\sum_{\mathrm{w}=1}^{\infty} \frac{\mathrm{w}\left(1-(-1)^{2^{\mathrm{u}^{2}}}\right)}{2}
$$

Here, when $u$ becomes 0 , the operator shall give 1 ; otherwise for any positive or negative integer it always shall give 0 ; so $\mathrm{w}=\mathrm{v}$ as the input number and $\mathrm{u}=\mathrm{m}-\mathrm{p}$ are used to realize this; but as 2,3,5 and 7 were included, the input number must be bigger than 7 .

\section{Integer floor function 2-3}

For $\mathrm{a} / \mathrm{b}$ operation where $\mathrm{a}, \mathrm{b} \in \mathbb{Z}$, when the result of $\mathrm{a}-\mathrm{bn}$ is positive integer for some $n$ positive integers, an equation must give +1 result in a sum operator. If the result is negative, the same equation must give 0 result in the same sum operator to find integer floor value of the division; so in Eq. (228) when $a-b m$ operation becomes negative, $f(z)$ must become an odd number, and otherwise must be an even number.

$$
\lfloor\mathrm{a} / \mathrm{b}\rfloor=\sum_{\mathrm{m}=1}^{\infty} \frac{1+(-1)^{\mathrm{f}(\mathrm{z})}}{2}
$$

Handle an operation like $\mathrm{x}(2 \mathrm{x}+1)$. If an order is made from small number to larger for all positive and negative $\mathrm{x}$ values where $\mathrm{x} \in \mathbb{Z}$, the number which has $\mathrm{n}$ order number becomes Eq. (229).

$$
\mathrm{N}=\sum_{\mathrm{n}=1}^{\mathrm{n}} \mathrm{n}-1
$$

For this condition, $\mathrm{N}$ numbers which have even order number in the emerging order are formed by negative x values; so $\mathrm{N}$ numbers which have odd order number in the emerging order are formed by positive $\mathrm{x}$ values.

To find out order number of the last $\mathrm{x}(2 \mathrm{x}+1)=\mathrm{N}_{\mathrm{n}}$ number for the input number of $\mathrm{x}$ where $\mathrm{n}$ is the order number, we need a reference. For example, we must always use either the previous number as $\mathrm{N}_{\mathrm{n}-1}$ or the next one as $\mathrm{N}_{\mathrm{n}+1}$; because if an addition is made by emerging $\mathrm{N}$ numbers by $\mathrm{N}_{\mathrm{m}}+\mathrm{N}_{\mathrm{n}-\mathrm{m}+1}$ being $n$ is the last order number and $m>0$, the difference also emerges by some consecutive odd numbers but some special conditions; so we need to know $\left(\mathrm{N}_{\mathrm{n}}+\mathrm{N}_{1}\right)-\left(\mathrm{N}_{\mathrm{n}-1}+\mathrm{N}_{2}\right)$; but each $\mathrm{N}$ number which has different order number is formed by different number; so even $\mathrm{x}_{1}=\mathrm{x}(2 \mathrm{x}+1)$ will give the last $\mathrm{N}$, $\mathrm{x}_{2}=-\mathrm{x}(-2 \mathrm{x}+1)$ does not always give only the previous one or only the next one for the same $\mathrm{x}$. This condition changes due to the mark of $\mathrm{x}_{\text {on }} \mathrm{x}_{1}$ and $\mathrm{x}_{2}$; so the order slides due to mark, and again a problem occurs; so to determine which one is the last one, again we must determine a rule between positive and nagative numbers that the main aim was already this to do these calculations.

\section{Floor function over the variations directly}

Right this point, if order number of positive and negative odd integers are analysed over only either by $\frac{\mathrm{r}+1}{2}$ which is order number formula of positive odd integers or $\frac{1-r}{2}$ which is order number of negative integers that handle $\frac{r+1}{2}$ is used, it seems that order number becomes odd number as the same r odd number's the other marked twin shall have even number order number; so

$$
\begin{aligned}
& e=\left\lfloor\frac{c+1}{2}\right\rfloor \\
& \mathrm{f}=\left\lfloor\frac{\mathrm{d}+1}{2}\right\rfloor
\end{aligned}
$$

will be the same number but the mark where $-\mathrm{y}=2 \mathrm{~g}-1$ $\mathrm{y}=2 \mathrm{~h}-1,-\mathrm{y}-\mathrm{g}=\mathrm{c}$ and $\mathrm{y}-\mathrm{h}=\mathrm{d}$; so over $\mathrm{y}-\frac{\mathrm{y}+1}{2}$ which is the single function of $\mathrm{c}$ and $\mathrm{d}$ over $\mathrm{y}=2 \mathrm{~h}-1$ and $-\mathrm{y}=2 \mathrm{~g}-1$, over Eq. (230a),

$$
\begin{aligned}
& \mathrm{f}(\mathrm{y})=\frac{\mathrm{y}+1-2(-1)^{\frac{\mathrm{y}-1}{2}}}{4} \\
& \mathrm{f}(\mathrm{y})=\frac{\frac{\mathrm{y}-1}{2}}{2}+\frac{1-(-1)^{\frac{\mathrm{y}-1}{2}}}{2}
\end{aligned}
$$

Eq. (230) shall provide this condition alone; but emerging results by an order is $0,1,1,2,2,3,3 \ldots$ To be of it as $1,2,3,4,5 \ldots$, if an order is created by doing an addition for each consecutive $f(y)$ and $f(y+2)$, required addition for the 
ordered $\mathrm{f}(\mathrm{y})$ result is $1,1,2,2,3,3,4,4 \ldots$ by order; so by using a function like $\mathrm{f}(\mathrm{t})=\frac{3+(-1)^{\mathrm{t}}}{2}$ which gives 2 for each even $\mathrm{k}$ number, and gives 1 for each $\mathrm{k}$ odd number where $\mathrm{k}=\mathrm{f}(\mathrm{y})+\mathrm{f}(\mathrm{y}+2)$,

$$
\mathrm{f}(\mathrm{k})=\frac{\mathrm{k}+\frac{3+(-1)^{\mathrm{k}}}{2}}{2}
$$

Eq. (230b) shall give the required number which will be added by order, and the result of $\mathrm{f}(\mathrm{y})$ turns into $1,2,3,4,5 \ldots$ as it is required as well; so for each $\pm \mathrm{y}$ even number, the order number turns into a positive odd number's order number by $\mathrm{f}(\mathrm{r})=\frac{\mathrm{r}+1}{2}$. The emerging sliding for the same oder function by using both variation of the same number as positive and negative is removed by this way.

Hence over $y \cdot f(k)$, a function which is equal to $y \cdot f(k)$ for positive $y$ odd numbers as $\mathrm{f}^{2}(\mathrm{k})+\mathrm{y}-1$ is also not equal to $y \cdot f(k)$ for the same number's negative variation; an operation like $\mathrm{M}$ is always even if $\mathrm{y}$ is negative, and is always odd if $\mathrm{y}$ is positive.

$$
\mathrm{M}=\frac{\mathrm{y} \cdot \mathrm{f}(\mathrm{k})+\mathrm{f}^{2}(\mathrm{k})+\mathrm{y}-1}{2}
$$

Hence, one variation of the floor function turns into Eq. (231),

$$
\lfloor\mathrm{a} / \mathrm{b}\rfloor=\sum_{\mathrm{m}=1}^{\infty} \frac{1-(-1)^{\mathrm{M}}}{2}
$$

where $y=2(a-b m)+1$. The upper limit for the sum can be for example $(a-b)^{2}$ instead of infinity as a reference.

\section{The other variation of the floor function}

To derive the previous $\mathrm{N}$ number by an input $\mathrm{x}$, for $+\mathrm{x}$, a function must give $-\mathrm{x}$ result, and for $-\mathrm{x}$ the same function must give $-x-1$. Temporarily, let assuming these results are provided by $\mathrm{f}(\mathrm{u})$.

For $\mathrm{N}_{\mathrm{m}}+\mathrm{N}_{\mathrm{n}-\mathrm{m}+1}$ addition being $\mathrm{n}$ is the last order number and $\mathrm{m}>0$, as we only use the last $\mathrm{N}$ and the previous one, $\mathrm{N}_{1}=0$ and $\mathrm{N}_{2}=1$ are always fixed. As the difference between two consecutive $\mathrm{N}$ number is always integers by order, for the fixed $\mathrm{N}$ numbers, the addition of the last one as $\mathrm{N}_{\mathrm{n}}+\mathrm{N}_{1}$ will be even number when the previous addition as $\mathrm{N}_{\mathrm{n}-1}+\mathrm{N}_{2}$ becomes odd; otherwise the condition is the exact opposite. When $d=x(2 x+1)+0-(f(u)(2 f(u)+1)+1)$ becomes even number, there is no middle point number as single in the new emerging set if you assume that $\mathrm{N}_{\mathrm{m}}+\mathrm{N}_{\mathrm{n}-\mathrm{m}+1}$ is done for each element; but otherwise, there is a single middle point number that the addition is done by itself; so if the difference $d$ is an even number, the last $\mathrm{N}$ number's order number becomes $n=d+2$. If the difference $d$ is an odd number, the last $\mathrm{N}$ number's order number becomes $\mathrm{n}=\mathrm{d}+3$. To write this in a single function, it becomes

$$
\mathrm{n}=\mathrm{d}+2+\frac{1-(-1)^{\mathrm{d}}}{2}
$$

As $f(u)$ function, the function must provide that for negative $\mathrm{x}$ values -1 is added to $-\mathrm{x}$, otherwise 0 is added to $-\mathrm{x}$. For this condition, it turns into Eq. (232a) where M is Eq. (230c).

$$
f(u)=-x+\frac{-(-1)^{\mathrm{M}}-1}{2}
$$

where $\mathrm{x}=\mathrm{y}$ in Eq. (230c).

Hence as order of $x(2 x+1)$ where $x \in \mathbb{Z}$, is due to from small to larger number by order, and as it starts by 0 which is formed by positive integer, if $\mathrm{n}$ which is the order number in emerging $\mathrm{N}$ set by this rule, becomes an even number, it means $\mathrm{x}$ is negative; otherwise $\mathrm{x}$ is positive; so the floor function turns into Eq. (233) over Eq. (228) for the required function on Eq. (228).

$$
\lfloor\mathrm{a} / \mathrm{b}\rfloor=\sum_{\mathrm{m}=1}^{\infty} \frac{1+(-1)^{\mathrm{n}}}{2}
$$

where $\mathrm{x}=\mathrm{a}-\mathrm{bm}$

\section{Integer floor function 4}

$$
\lfloor\mathrm{a} / \mathrm{b}\rfloor=\sum_{\mathrm{m}=1}^{\infty} \frac{1-(-1)^{2}{ }^{\left(\Pi_{\mathrm{n}=1}^{\infty} \mathrm{x}_{\mathrm{n}}\right)^{2}}}{2}
$$

where $\mathrm{x}_{0}=\mathrm{a}-\mathrm{bm}$ and $\mathrm{x}_{\mathrm{n}}=\frac{2 \mathrm{x}_{\mathrm{n}-1}-1+(-1)^{\mathrm{x}_{\mathrm{n}-1}}}{4}$. The upper limit for both of the product and the sum can be for example $(a-b)^{2}$ instead of infinity as a reference.

\section{Integer floor function 5}

Any odd number increases only by Eq. (235) for its even multiples,

$$
\mathrm{f}_{1}(\mathrm{x}, \mathrm{y})=(4 \mathrm{y}+2) \mathrm{x}
$$

and only increases by Eq. (236) for its odd multiples.

$$
\mathrm{f}_{2}(\mathrm{x}, \mathrm{y})=(4 \mathrm{y}+2) \mathrm{x}-(2 \mathrm{y}+1)
$$

It is such; because for an equation like Eq. (237)

$$
2 y=2 x^{2}+2 x-1
$$

over $(2 \mathrm{x}+1)+2 \mathrm{y}=(\mathrm{x}+1)(2 \mathrm{x}+1)$, as $2 \mathrm{x}^{2}+2 \mathrm{x}$ is always an even number, it can be said, that there is no consecutive multiples of the same odd number for the functions in itself as even after odd or odd after even, as numbers increase by 2 . There is no intersection point between Eq. (235) and Eq. (236).

To find out number of an odd number up to $n$ number, without decimal by a floor function, the equations become Eq. (238) and Eq. (239),

$$
\begin{aligned}
& \mathrm{x}_{1}=\left\lfloor\frac{\mathrm{n}}{2(2 \mathrm{y}+1)}\right\rfloor \\
& \mathrm{x}_{2}=\left\lfloor\frac{\mathrm{n}+2 \mathrm{y}+1}{2(2 \mathrm{y}+1)}\right\rfloor
\end{aligned}
$$

where $\mathrm{n}$ is the input number. Here, the main problem is validation of Eq. (240) or Eq. (241) if the first operation is $\left\lfloor\frac{n}{2}\right\rfloor$ or $\left\lfloor\frac{\mathrm{n}+2 \mathrm{y}+1}{2}\right\rfloor$.

$$
\begin{gathered}
\mathrm{x}_{1}=\left\lfloor\left\lfloor\frac{\mathrm{n}}{2}\right\rfloor \cdot \frac{1}{2 \mathrm{y}+1}\right\rfloor \\
\mathrm{x}_{2}=\left\lfloor\left\lfloor\frac{\mathrm{n}+2 \mathrm{y}+1}{2}\right\rfloor \cdot \frac{1}{2 \mathrm{y}+1}\right\rfloor
\end{gathered}
$$

For real value of $\frac{\mathrm{n}}{2}$, over Eq. (242a), it becomes Eq. (242).

$$
\begin{gathered}
\mathrm{n}=8 \mathrm{y}+4 \\
1=\frac{\frac{\mathrm{n}}{2}-(2 \mathrm{y}+1)}{2 \mathrm{y}+1}
\end{gathered}
$$


Here Eq. (242a) is decimal approach. As it can be seen over Eq. (242) which is the result of Eq. (242a), there is no integer interval for the $y$ numbers which are integer. As $n$ and $y$ take certain values, there shall not emerge a skipping; so there is no difference between $\left\lfloor\left\lfloor\frac{n}{2}\right\rfloor \cdot \frac{1}{2 y+1}\right\rfloor$ and $\left\lfloor\frac{1}{2} \cdot\left\lfloor\frac{n}{2 y+1}\right\rfloor\right\rfloor$ for operation priority.

In the same manner, the equation becomes Eq. (243) for $\left\lfloor\frac{n+2 y+1}{2}\right\rfloor$ over Eq. (243a); so the same is acceptable.

$$
\begin{gathered}
\mathrm{n}=6 \mathrm{y}+3 \\
1=\frac{\frac{\mathrm{n}+2 \mathrm{y}+1}{2}-(2 \mathrm{y}+1)}{2 \mathrm{y}+1}
\end{gathered}
$$

Hence, the equation turns into Eq. (244),

$$
\mathrm{x}_{1}=\left\lfloor\frac{\mathrm{n}_{1 \mathrm{x}_{1}}}{2 \mathrm{y}+1}\right\rfloor
$$

where $n_{1 x_{1}}=\left\lfloor\frac{n}{2}\right\rfloor$. This turned into a single odd number's separation by its all multiples even multiples are included as well; because actual question is how many $(2 \mathrm{y}+1)$ odd number there are between 0 and n, before deriving Eq. (238) and Eq. (239); so it is depended on $\left\lfloor\frac{\mathrm{n}}{2 \mathrm{y}+1}\right\rfloor$ since the actual equation is Eq. (245).

$$
\left\lfloor\frac{\mathrm{n}}{2 \mathrm{y}+1}\right\rfloor=\left\lfloor\frac{\mathrm{n}}{2(2 \mathrm{y}+1)}\right\rfloor+\left\lfloor\frac{\mathrm{n}+2 \mathrm{y}+1}{2(2 \mathrm{y}+1)}\right\rfloor
$$

Hence again it can be used as Eq. (246) and Eq. (247) for the same y number.

$$
\begin{gathered}
\mathrm{x}_{1_{1}}=\left\lfloor\frac{\mathrm{n}_{1 \mathrm{x}_{1}}}{2(2 \mathrm{y}+1)}\right\rfloor \\
\mathrm{x}_{1_{2}}=\left\lfloor\frac{\mathrm{n}_{1 \mathrm{x}_{1}}+2 \mathrm{y}+1}{2(2 \mathrm{y}+1)}\right\rfloor
\end{gathered}
$$

The same is acceptable for Eq. (239),

$$
\begin{aligned}
& \mathrm{x}_{2_{1}}=\left\lfloor\frac{\mathrm{n}_{2 \mathrm{x}_{2}}+2 \mathrm{y}+1}{2(2 \mathrm{y}+1)}\right\rfloor \\
& \mathrm{x}_{2_{2}}=\left\lfloor\frac{\mathrm{n}_{2 \mathrm{x}_{2}}+4 \mathrm{y}+2}{2(2 \mathrm{y}+1)}\right\rfloor
\end{aligned}
$$

where $\mathrm{n}_{2 \mathrm{x}_{2}}=\left|\frac{\mathrm{n}+2 \mathrm{y}+1}{2}\right|$; so for both Eq. (238) and Eq. (239), a continuous chain rule emerges up to infinite.

The above stated information are valid even for even numbers. I wanted to show it over odd numbers; because it is important to see the divisibility condition of 2 in a floor function. Hence for any kind positive integer numerator and denominator, the main equation turns into Eq. (250) by the presentation of Eq. (250a).

$$
\begin{gathered}
\left\lfloor\frac{a}{b}\right\rfloor=\left\lfloor\frac{a}{2 b}\right\rfloor+\left\lfloor\frac{a+b}{2 b}\right\rfloor \\
\lfloor d\rfloor=\lfloor e\rfloor+\lfloor f\rfloor
\end{gathered}
$$

Over Eq. (250), the first process for the equation becomes
Eq. (250b) by the presentation of Eq. (250c),

$$
\begin{gathered}
\left\lfloor\frac{a}{b}\right\rfloor=\left(\left\lfloor\frac{a_{1}}{2 b}\right\rfloor+\left\lfloor\frac{a_{1}+b}{2 b}\right\rfloor\right)+\left(\left\lfloor\frac{a_{2}}{2 b}\right\rfloor+\left\lfloor\frac{a_{2}+b}{2 b}\right\rfloor\right) \\
\lfloor d\rfloor=\left(\left\lfloor e_{1}\right\rfloor+\left\lfloor e_{2}\right\rfloor\right)+\left(\left\lfloor f_{1}\right\rfloor+\left\lfloor f_{2}\right\rfloor\right)
\end{gathered}
$$

where $a_{1}=\left\lfloor\frac{a}{2}\right\rfloor$. Here, if the right side of the equation is multiplied by 2 , it turns into Eq. (250d).

$$
2\lfloor\mathrm{~d}\rfloor=2\left(\left\lfloor\mathrm{e}_{1}\right\rfloor+\left\lfloor\mathrm{e}_{2}\right\rfloor\right)+2\left(\left\lfloor\mathrm{f}_{1}\right\rfloor+\left\lfloor\mathrm{f}_{2}\right\rfloor\right)
$$

Here, $e_{1}$ and $f_{1}$ were dived by 2 in floor function; so how we can write the right relation between $2\left\lfloor\frac{\lfloor\mathrm{a} / 2\rfloor}{2 \mathrm{~b}}\right\rfloor$ and $\left\lfloor\frac{\mathrm{a}}{2 \mathrm{~b}}\right\rfloor$, this is the problem; because when this is done, we can also write the equation in the kind of $\left\lfloor e_{2}\right\rfloor$ and $\left\lfloor f_{2}\right\rfloor$; so for an operation like $\left\lfloor\frac{\mathrm{u}}{\mathrm{t}}\right\rfloor$, the equation is Eq. (251),

$$
2\left\lfloor\frac{\lfloor\mathrm{u} / 2\rfloor}{\mathrm{t}}\right\rfloor=\left\lfloor\frac{\mathrm{u}}{\mathrm{t}}\right\rfloor
$$

if it is $\left\lfloor\frac{\mathrm{u}}{2}\right\rfloor=\mathrm{t}$ or $\left\lfloor\frac{\mathrm{u}}{2}\right\rfloor>\mathrm{t}$ by whole multiples of $\mathrm{t}$. For the last option as $\left\lfloor\frac{\mathrm{u}}{2}\right\rfloor<\mathrm{t}$ also it is still valid; but due to to be odd or even integer of $u$, as $2\left\lfloor\frac{\lfloor\mathrm{u} / 2\rfloor}{\mathrm{t}}\right\rfloor$ is always even number, when $\left\lfloor\frac{u}{t}\right\rfloor$ becomes odd number the equation does not work. Hence, the right interpretation is as Eq. (252),

$$
2\left\lfloor\frac{\lfloor\mathrm{u} / 2\rfloor}{\mathrm{t}}\right\rfloor+\frac{1-(-1)^{\lfloor\mathrm{u} / 2\rfloor}}{2}=\left\lfloor\frac{\mathrm{u}}{\mathrm{t}}\right\rfloor
$$

where $\left\lfloor\frac{\mathrm{u}}{2}\right\rfloor=\frac{\mathrm{u}}{2}-\frac{1-(-1)^{\mathrm{u}}}{4}$. Right this point, it turns into Eq. (253),

$$
\left\lfloor\mathrm{e}_{1}\right\rfloor=\frac{\lfloor\mathrm{e}\rfloor-\mathrm{k}}{2}
$$

where $\mathrm{k}=\frac{1-(-1)^{\lfloor\mathrm{a} / 2\rfloor}}{2}$. Hence, also Eq. (250d) includes $\lfloor\mathrm{e}\rfloor+$ $\lfloor f\rfloor$ instead of $\left\lfloor e_{1}\right\rfloor+\left\lfloor f_{1}\right\rfloor$, and it turns into Eq. (254).

$$
2\lfloor\mathrm{~d}\rfloor=\left(\lfloor\mathrm{e}\rfloor-\mathrm{k}+2\left\lfloor\mathrm{e}_{2}\right\rfloor\right)+\left(\lfloor\mathrm{f}\rfloor-\mathrm{m}+2\left\lfloor\mathrm{f}_{2}\right\rfloor\right)
$$

where $\mathrm{m}=\frac{1-(-1)^{\lfloor(\mathrm{a}+\mathrm{b}) / 2\rfloor}}{2}$. It means that the equation is Eq. (255).

$$
\lfloor d\rfloor=\lfloor e\rfloor+\lfloor f\rfloor=2\left(\left\lfloor e_{2}\right\rfloor+\left\lfloor f_{2}\right\rfloor\right)-k-m
$$

All right; but this is not enough; because we cannot know upper floor values in emerging infinite chain. We must know which chain lines include only whole multiples. Being these are the numbers like $\mathrm{e}_{1}=\mathrm{x}+\frac{1}{2}, \mathrm{f}_{1}=\mathrm{y}+\frac{1}{2}$ and thus $\mathrm{f}_{2}=\mathrm{f}_{1}+$ $\frac{1}{2}$ for the worst possibility, the equation becomes Eq. (256),

$$
\lfloor d\rfloor=\left\lfloor e_{1}+f_{2}\right\rfloor+\left\lfloor e_{2}+f_{1}\right\rfloor
$$

where $\left\lfloor e_{1}\right\rfloor+\left\lfloor f_{2}\right\rfloor=\left\lfloor e_{1}+f_{2}\right\rfloor$, and as the same is acceptable for $\left\lfloor e_{2}\right\rfloor+\left\lfloor f_{1}\right\rfloor=\left\lfloor e_{2}+f_{1}\right\rfloor$ also this is a certain rule which is always valid. It means that, for the chain operation which will emerge by the same method, it is possible to use only one double element from both side of $e$ and $f$ for any multitude elements.

For the first process of a floor function as $\lfloor e\rfloor+\lfloor f\rfloor$, if a table is made by emerging chain operations, being $\mathrm{E}$ is even 
multiples side and $\mathrm{O}$ is odd multiples side, the table turns into Table 5 by using middle elements of the emerging chain triangle by the same rule of Eq. (250b) when it is sustained.

\begin{tabular}{c|c|c|c}
$\mid$ & $\mathbf{E}$ & $\mathbf{O}$ & \\
\hline & $\mathrm{e}$ & $\mathrm{f}$ & \\
\hline$\cdot \mid \mathrm{f}_{2}$ & $\mathrm{e}_{2}$ & $\cdot$ \\
\hline$\cdot \mid \mathrm{f}_{\mathrm{a}}$ & $\mathrm{e}_{\mathrm{a}}$ & $\cdot$ \\
\hline$\cdot \mid \mathrm{f}_{\mathrm{b}}$ & $\mathrm{e}_{\mathrm{b}}$ & $\cdot$ \\
\hline$\cdot \mid \mathrm{f}_{\mathrm{c}}$ & $\mathrm{e}_{\mathrm{c}}$ & $\cdot$ \\
\hline$\cdot \mid \mathrm{f}_{\mathrm{d}}$ & $\mathrm{e}_{\mathrm{d}}$ & $\cdot$
\end{tabular}

Table 5: Double middle groups of e and $\mathrm{f}$

The used numbers are only subordinate neighbors of $\left\lfloor f_{2}+e_{2}\right\rfloor$. Here as it can be seen, the condition turns into the exact opposite; because even column started to include odd multiple formula, and odd column also includes even multiple formula. $\mathrm{O}$ column certainly shall become 0 on a line, and after that it will be always 0 . In the same manner, the other column shall be $2 \mathrm{~b}$ on a line, and after that, it will always include $2 \mathrm{~b}$. As we cannot know floor value of the higher elements of the table, we must know when sum of $\mathrm{E}+\mathrm{O}$ starts to repeat on each line by the same values to infinity. At this repeat point, it means there is 1 whole value; so if we can find out when the middle sum becomes whole multiple of the denominator certainly by the input values as denominator and numerator, we can use the process number up to this line. To determine this, we must always dive $\mathrm{f}$ by 2 down to 0 for floor value; so

$$
\mathrm{n}=1+\sum_{\mathrm{s}=0}^{\infty} \frac{1+(-1)^{2^{\mathrm{f}} \mathrm{s}+1}}{2}
$$

where $\mathrm{f}_{\mathrm{s}+1}=\frac{2 \mathrm{f}_{\mathrm{s}}-1+(-1)^{\mathrm{f}_{\mathrm{s}}}}{4}$ and $\mathrm{f}_{0}=\mathrm{f}$. This is the process number which is required to make $\mathrm{f}$ number 0 by diving only by 2 for each operation's floor value.

At the line which the repeat starts, there is absolutely 1 whole value for each column of both columns as numerator and denominator are equal to each other. Process number is used to determine whole value right this point. The first equation is $\frac{\lfloor d\rfloor+k+m}{2}=\left\lfloor e_{2}\right\rfloor+\left\lfloor f_{2}\right\rfloor$, and it converges to the last e-f double by

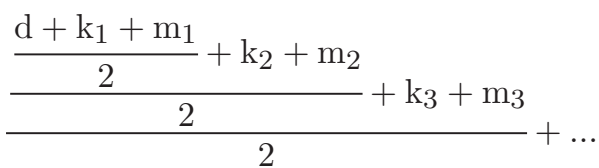

Hence the floor function becomes Eq. (258) being $n$ is the process number.

$$
\left\lfloor\frac{\mathrm{a}}{\mathrm{b}}\right\rfloor=2^{\mathrm{n}}\left(\mathrm{e}_{\mathrm{n}}+\mathrm{f}_{\mathrm{n}}\right)-\sum_{\mathrm{n}=1}^{\mathrm{n}} 2^{\mathrm{n}-1}\left(\mathrm{k}_{\mathrm{n}}+\mathrm{m}_{\mathrm{n}}\right)
$$

By the way, here actually each one of the last e and $f$ values are 1 if the numerator is not smaller than the denominator. Also actually always second e and $\mathrm{f}$ values are taken; but as the other doubles are removed, we can assume that they are the first ones. Also to find out each $\mathrm{k}_{\mathrm{n}}$ and $\mathrm{m}_{\mathrm{n}}$,

$$
\mathrm{k}_{\mathrm{n}}=\sum_{\mathrm{n}=1}^{\mathrm{n}} \frac{1-(-1)^{\mathrm{w}_{\mathrm{n}-1}}}{2}
$$

where $\mathrm{w}_{\mathrm{n}}=\left\lfloor\frac{\mathrm{w}_{\mathrm{n}-1}+3}{2}\right\rfloor=\frac{2\left(\mathrm{w}_{\mathrm{n}-1}+3\right)-1+(-1)^{\mathrm{w}} \mathrm{n}-1+3}{4}$, and $\mathrm{w}_{0}=\left\lfloor\frac{\mathrm{a}}{2}\right\rfloor=\frac{2 \mathrm{a}-1+(-1)^{\mathrm{a}}}{4}$.

$$
\mathrm{m}_{\mathrm{n}}=\sum_{\mathrm{n}=1}^{\mathrm{n}} \frac{1-(-1)^{\mathrm{z}_{\mathrm{n}-1}}}{2}
$$

where $z_{n}=\left\lfloor\frac{z_{n-1}+3}{2}\right\rfloor=\frac{2\left(z_{n-1}+3\right)-1+(-1)^{z_{n}-1+3}}{4}$ and $z_{0}=$ $\left\lfloor\frac{\mathrm{a}+\mathrm{b}}{2}\right\rfloor=\frac{2(\mathrm{a}+\mathrm{b})-1+(-1)^{\mathrm{a}+\mathrm{b}}}{4}$.

\subsection{On the Mersenne and Fermat primes}

\subsubsection{Mersenne primes}

Mersenne primes, which are depended on Eq. (259) does not give always prime result.

$$
2^{\mathrm{p}_{1}-1}=\mathrm{p}_{2}
$$

If Eq. (259) is turned into Eq. (259a),

$$
2=\sqrt[p_{1}]{\mathrm{p}_{2}+1}
$$

then $\left(\mathrm{p}_{2}+1\right)$ always must be a number like $2^{\mathrm{n}}$. If this is the condition, then for $\mathrm{p}_{2}=2^{\mathrm{n}}-1$ where $\mathrm{n} \geq 1$ positive integers, the result always must be prime number.

$2^{\mathrm{n}}-1$ is always a positive odd number and is always prime number, where $\left\{n \in \mathbb{Z}^{+} \mid 0<n\right\}$.

\section{Argument}

Let using a function like $\mathrm{f}(\mathrm{x})=10 \mathrm{x}+3$. If there were infinite number of intersection points of $2^{\mathrm{n}}-1$ with $\mathrm{f}(\mathrm{x})$, then $2^{\mathrm{n}}-1$ would not always become a prime number. For $2^{\mathrm{n}}-1=$ $10 \mathrm{x}+3$ equation, it turns into Eq. (260).

$$
\mathrm{x}=\frac{2^{\mathrm{n}-1}-2}{5}
$$

For some $n>2$ positive integers forever, Eq. (260) gives $\mathrm{x}$ integers forever as 5 or multiples of 5 when $2^{\mathrm{n}-1}$ had 2 in the first digit as $2^{\mathrm{n}-1}$ has a constant repeat as $2^{\mathrm{n}-1}$ can only be formed by 2 . If this is the condition, then it explains that Mersenne prime function doesn't give always prime results for given variable primes.

\subsubsection{Fermat primes}

$2^{\mathrm{n}}+1$ is always a positive odd number and is always prime number, where $\left\{n \in \mathbb{Z}^{+} \mid 0<n\right\}$.

\section{Argument}

In the same manner, let using a function like $\mathrm{f}(\mathrm{x})=10 \mathrm{x}+$ 5. For $2^{\mathrm{n}}+1=10 \mathrm{x}+5$ equation, it turns into Eq. (261)

$$
\mathrm{x}=\frac{2^{\mathrm{n}}-2}{5}
$$

Eq. (261) has the same result with Eq. (260); so it can be said that Fermat prime function which is depended on Eq. (261a) 
does not always give primes where $2^{2^{\mathrm{m}}}=2^{\mathrm{k}}$.

$$
\mathrm{p}=2^{2^{\mathrm{m}}}+1
$$

As the result, as Mersenne and Fermat prime functions don't give always prime result consecutively; so they may give prime results by variable intervals forever.

\subsubsection{A common solution}

As any prime number is between two odd positive consecutive multiples of 3 , let using $f(x)=6 x+5$ function which is always between two positive odd multiples of 3 , where $\left\{\mathrm{x} \in \mathbb{Z}^{+} \mid 0<\mathrm{x}\right\}$, and is always the next one relatively to the smaller multiple of two consecutive multiples of 3 . For $2^{\mathrm{a}}-1=6 \mathrm{x}+5$ equation, it turns into Eq. (262).

$$
\mathrm{x}=\frac{2^{\mathrm{a}-1}}{3}+1
$$

Here, as $2^{\mathrm{a}-1}$ is only be formed by 2 , it is not dividable by 3 ; so $\mathrm{x}$ on Eq. (262) is always decimal, and $2^{\mathrm{a}-1}$ can never exist as the next odd number after 3 . Also as non-prime numbers or prime numbers cannot only exist alone as the previous odd number just before 3 or after 3 as the next one consecutively as we can see on Eq. (264) and Eq. (264a), Mersenne function always may give some prime results forever as well. $f(x)=$ $6 \mathrm{x}+7$ function is always between two positive odd multiples of 3 for positive $x$ numbers and is always the previous one. For $2^{\mathrm{b}}+1=6 \mathrm{x}+7$, it turns into Eq. (262a),

$$
\mathrm{x}=\frac{2^{\mathrm{b}-1}}{3}-1
$$

and as $2^{\mathrm{b}-1}$ is only formed by 2 , it is not dividable by 3 ; so $\mathrm{x}$ on Eq. (262a) is always decimal and $2^{\mathrm{b}}+1$ can never exist as the previous odd number before 3 . Also as non-prime numbers or prime numbers cannot only exist alone as the previous odd number just before 3 or after 3 as the next one consecutively as we can see on Eq. (264) and Eq. (264a), Fermat function always may give some prime results forever as well.

Even if Fermat and Mersenne prime functions certainly do not give prime results always, we cannot know if they give non-prime result. This was proved in Section 7.7.

\section{Notice}

\subsection{Palindromic primes}

Are there infinitely many palindromic primes?

Argument

\subsubsection{Analysis of first degree function possibility of prime numbers}

As we know, the palindromic primes are the prime numbers which give the same number when they are written from left to right or right to left. Infinity of them is still a mystery; but we can prove that they are also in infinite number.

As we know, distribution of odd multiples of 3 in the set of odd numbers occurs according to $f(x)=6 x-3$ function where $\mathrm{x}>0$ for each consecutive positive $\mathrm{x}$ integer; so if a table is made for the definition, it occurs like the following one.

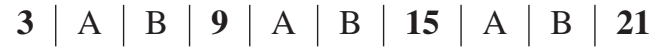

Table 6: Distribution of odd multiples of 3 for a limited interval. Here $\mathrm{A}$ is the numbers occur after the multiples of 3 , and $\mathrm{B}$ is the numbers occur before the multiples of 3 since there are only 2 odd number between each consecutive odd multiple of 3 in the set of odd numbers.

Since each prime number is an element of the set of odd numbers, they exactly take place between two consecutive odd multiples of 3 being before or after the multiples.

For separation rule of 7 by $f(a)=14 a+7$ and of 5 by $\mathrm{f}(\mathrm{b})=10 \mathrm{~b}+5$ in odd numbers, if intersection points of $\mathrm{f}(\mathrm{a})$ and $\mathrm{f}(\mathrm{b})$ constantly appeared as only the previous one or only as the next one of odd multiples of 3 , then an uncertainty would emerge about first degree function possibility of prime numbers by certain intervals. For $14 \mathrm{a}+7=10 \mathrm{~b}+5$ equation, it becomes Eq. (263).

$$
\mathrm{b}=\frac{7 \mathrm{a}+1}{5}
$$

Hence, when $7 \mathrm{a}+1$ becomes 0 or 5 for its first digit, b becomes an integer and also an intersection point; so for this condition, a directly becomes dependent of $\mathrm{f}(\mathrm{c})=5 \mathrm{c}-3$ function. Each c positive integer gives a result an integer to make $\mathrm{b}$ an integer, and to create an intersection point between multiples of 5 and 7. For each c integer value, if the emerging intersection point is always the previous or is the next one relatively to odd multiples of 3 , then an uncertainty is going to emerge about first degree function possibility of prime numbers by certain intervals; so to analysis this, for the function $\mathrm{f}(\mathrm{d})=6 \mathrm{~d}+5$ which gives the next odd numbers after odd multiples of 3 , over $\mathrm{f}(\mathrm{b})$ which is $\mathrm{f}(\mathrm{b})=10\left(\frac{7 \mathrm{f}(\mathrm{c})+1}{5}\right)+5$, it becomes Eq. (263a) for $f(b)=f(d)$ equation.

$$
\mathrm{d}=\frac{35 \mathrm{c}-20}{3}
$$

Hence, it can be said, that d is not defined for each c consecutive positive integer; so the intersection point of 5 and 7 in odd numbers sometimes appears at the place after odd multiples of 3, and sometimes appears before; so as there are only two odd integer places between two consecutive odd multiples of 3 , if it is derived a first degree function which is always only able to appear before or after odd multiples of 3 , the numbers which are placed here cannot be prime number always as also they cannot be non-prime odd number.

\subsubsection{The solution}

All the positive odd numbers before odd multiples of 3 were non-prime numbers, then as all positive odd numbers are between two consecutive odd multiples of 3 and there are two odd numbers between them, all the positive odd numbers after odd multiples of 3 would have to be prime number; but it is not possible as there is always going to be multiples of 7 as far as we can see over Eq. (264a), and also because of first degree impossibility of prime numbers as the below.

Hence, for example by using positive integers for between two consecutive positive odd multiples of $3, \mathrm{f}(\mathrm{x})=6 \mathrm{x}+5$ function is always between these two positive odd multiples of 3 , where $\left\{x \in \mathbb{Z}^{+} \mid 0<x\right\}$, and is always the next one. If we use the separation rule of 5 , then for $6 \mathrm{x}+5=10 \mathrm{y}-5$ 
equation, it turns into Eq. (264) .

$$
\mathrm{y}=\frac{3 \mathrm{x}}{5}+1
$$

For positive integer $\mathrm{u}$ numbers and $\mathrm{x}=5 \mathrm{u}$ equation, $\mathrm{x}$ on Eq. (264) is always going to form y as an integer; so there are always some positive odd multiples of 5 at the next positions of positive odd multiples of 3 . For the second and last state, $\mathrm{f}(\mathrm{x})=6 \mathrm{x}+7$ gives the previous odd numbers of odd multiples of 3 . If this is the condition, then by using $6 x+7=14 x-7$ equation as odd multiples of 7 which is dependent of $\mathrm{f}(\mathrm{y})=$ $14 y-7$ function, it turns into Eq. (264a).

$$
y=\frac{3 x}{7}+1
$$

For positive integer $\mathrm{u}$ numbers and $\mathrm{x}=7 \mathrm{u}$ equation, $\mathrm{x}$ on Eq. (264a) is always going to form y as an integer; so there are always some positive odd multiples of 7 at the previous positions of positive odd multiples of 3 . If this is the condition, then there are infinite numbers of palindromic primes by the rule of odd multiple of 3 which is $500 \ldots 007$ has variable number of zeroes or other symmetric numbers which their sum is multiple of 3 between 5 and 7 , and as prime 7 and 7 . Also you can derive your own sexy primes as you can prove for the numbers have variable number of 0 between 1 and 1 .

\subsubsection{Palindromic primes with number of digits}

\section{Wilson's Theorem}

The theorem of Wilson states, that if $\mathrm{p}$ is a prime number, then this prime number divides $(\mathrm{p}-1) !+1$ being the result is an integer. above.

The following formula Eq. (265) is the one I derived

$$
\mathrm{e}=\lim _{\mathrm{n} \rightarrow \infty} \frac{\mathrm{n}}{\sqrt[n+1]{(\mathrm{n}+1) !}}
$$

Now handle the palindromic primes which can be written as Eq. (266) where the number of digits of the prime $\mathrm{p}$ is $10^{\mathrm{m}}$ being $\mathrm{m} \in \mathbf{Z}$.

$$
\mathrm{p}=10^{10^{\mathrm{m}}}+1
$$

If the result of Eq. (267) operation must be an integer, then we can edit it for the solutions.

$$
\frac{(\mathrm{p}-1) !+1}{\mathrm{p}}
$$

Over $\frac{p !(p-1) !+p !}{p^{2}(p-1) !}$ operation which is equivalent of Eq. (267), Eq. (267) turns into Eq. (268).

$$
\frac{\mathrm{p} !+\mathrm{p}}{\mathrm{p}^{2}}
$$

For the greatest enough $\mathrm{n}$ value if Eq. (265) is edited, it becomes Eq. (269) where $\mathrm{p}=\mathrm{n}$ and e is Euler number.

$$
\mathrm{p} !=\frac{1}{\mathrm{p}+1}\left(\frac{\mathrm{p}}{\mathrm{e}}\right)^{\mathrm{p}+1}
$$

If Eq. (269) is used on Eq. (268), it becomes Eq. (270)

$$
\mathrm{i}=\frac{1}{\mathrm{p}^{2}}\left(\frac{\mathrm{p}-1}{\left(1+\frac{1}{\mathrm{p}-1}\right)^{\mathrm{p}-1}}\right)^{\mathrm{p}}+\frac{1}{\mathrm{p}}
$$

or becomes Eq. (271) if you assume that e converges to the same value by the same $n$ in both Eq. (265) and $\lim _{n \rightarrow \infty}(1+$ $\left.\frac{1}{n}\right)^{n}$

$$
\mathrm{p}\left(\mathrm{ip}^{2}-\mathrm{p}\right)^{\frac{1}{\mathrm{p}(\mathrm{p}-1)}}=(\mathrm{p}-1)^{\frac{\mathrm{p}}{\mathrm{p}-1}}
$$

Now how we can fix the deviation between Eq. (265) and $\lim _{\mathrm{n} \rightarrow \infty}\left(1+\frac{1}{\mathrm{n}}\right)^{\mathrm{n}}$. Is it suitable to use e with enough decimal? Even if it is possible, also is there anyway to reduce process number of the function for Eq. (266), especially for $\mathrm{m}>8$.

\section{Fermat's Little Theorem}

The theorem of Fermat states, that if $\mathrm{p}$ is a prime number, then this prime number divides $\mathrm{n}^{\mathrm{p}-1}-1$ being the result is an integer where $n \in \mathbf{N}$; so for $n=2$, Eq. (272) must give integer result for Eq. (266).

$$
\frac{2^{10^{10^{\mathrm{m}}}}-1}{10^{10^{\mathrm{m}}}+1}
$$

Also is there anyway to simplify this method especially for $\mathrm{m}>8$ and maybe for $\mathrm{n}=10$ ?

\subsection{Goldbach Conjecture}

The main question about Goldbach Conjecture is pretty clear that is each even number sum of two prime numbers?

\subsubsection{The solution}

Being $\mathrm{p}$ is a prime number, for definition of $\mathrm{p}_{4}>\mathrm{p}_{3} \wedge$ $\mathrm{p}_{4}-\mathrm{p}_{3}=2 \mathrm{n}+2 \wedge \mathrm{n}>2 \wedge \mathrm{n} \in \mathbb{Z}^{+}$; first number group which is created by $n$ pieces non-prime consecutive positive odd whole numbers, the smallest odd whole number it has is $\mathrm{p}_{3}+2$ or $\mathrm{p}_{4}-2 \mathrm{n}$ and the biggest odd number it has is $\mathrm{p}_{3}+2 \mathrm{n}$ or $\mathrm{p}_{4}-2$, contains greater numbers than last number group which contains $n-1$ pieces consecutive non-prime odd numbers and none of the numbers $n$ group contains, for definition of $p_{2}>$ $\mathrm{p}_{1} \wedge \mathrm{p}_{2}-\mathrm{p}_{1}=2(\mathrm{n}-1)+2$ is before $\mathrm{n}$ group, and the smallest odd number it has is $\mathrm{p}_{1}+2$ or $\mathrm{p}_{2}-2(\mathrm{n}-1)$ and the biggest one it has is $\mathrm{p}_{1}+2(\mathrm{n}-1)$ or $\mathrm{p}_{2}-2$. Also $\mathrm{n}-1$ groups which contain $\mathrm{n}-1$ pieces non-prime consecutive odd numbers and have greater numbers than $\mathrm{n}$ groups have are possible; but these groups can never exist until $n$ groups emerge because of the above stated reasons and definitions; because $\mathrm{n}$ groups also contain 2 pieces $(n-1)_{1}$ and $(n-1)_{2}$ consecutive groups which $\mathrm{n}-2$ pieces elements of them are common with $\mathrm{n}$ groups.

The difference between $n-1$ and $(n-1)_{m}$ is not to be of prime number there after last number of first group and before first number of last group of emerging two groups; therefore when two groups emerged as $(n-1)_{1}$ and $(n-1)_{2}$, it means that $\mathrm{n}$ group already had been emerged spontaneously, and is first $n$ group. Being $(n-1)_{1}$ is the first and $(n-1)_{2}$ is the second group, as last element of $(n-1)_{2}$ group is always bigger than all numbers of $(n-1)_{1}$, it means $(n-1)_{1}$ had already been emerged before $n$ group. Also as $n-1$ group must exist at the place before $(n-1)_{1}$ group, then numbers of $n$ group are always greater than numbers of $n-1$ group for the definition of $n$ and $n-1$ groups. Here all possible $n-k$ groups for 
$\mathrm{m}, \mathrm{k} \in \mathbb{Z}^{+}$definition are unique; but $(\mathrm{n}-\mathrm{k})_{\mathrm{m}}$ groups which have common element with another groups are not unique.

Being $\mathrm{k}=0$, for $\mathrm{n}-\mathrm{k}=\mathrm{n}$ groups, 1 piece of selected $\mathrm{n}$ pieces consecutive odd numbers from 3 to numbers of $n+1$ group has to be prime number; so if some tables are made like table 7 and table 8 for each $n$, they will help about the main question.

Table 7: The table for $n=2$

\begin{tabular}{l|lllllll}
\hline $\mathbf{x}$ & & & & $\mathbf{y}$ & & & \\
\hline 3 & 3 & 5 & 7 & 9 & 11 & 13 & $\ldots$ \\
5 & 5 & 7 & 9 & 11 & 13 & 15 & $\ldots$ \\
\hline & $\mathbf{x}$ & + & $\mathbf{y}$ & $=$ & $\mathbf{b}$ & & \\
\hline & 6 & 8 & $\mathbf{1 0}$ & $\mathbf{1 2}$ & 14 & $\ldots$ & $2 \mathrm{a}+4$ \\
& $\mathbf{1 0}$ & $\mathbf{1 2}$ & 14 & 16 & 18 & $\ldots$ & $2 \mathrm{a}+8$ \\
\hline
\end{tabular}

Table 8: The table for $n=3$

\begin{tabular}{l|llllllll}
\hline $\mathbf{x}$ & \multicolumn{1}{c}{$\mathbf{y}$} \\
\hline 3 & 3 & 5 & 7 & 9 & 11 & 13 & $\ldots$ & \\
5 & 5 & 7 & 9 & 11 & 13 & 15 & $\ldots$ & \\
7 & 7 & 9 & 11 & 13 & 15 & 17 & $\ldots$ & \\
\hline & $\mathbf{x}$ & + & $\mathbf{y}$ & $=$ & $\mathbf{b}$ & & & \\
\hline & 6 & 8 & 10 & 12 & $\mathbf{1 4}$ & $\mathbf{1 6}$ & $\ldots$ & $2 \mathrm{a}+4$ \\
& 10 & 12 & $\mathbf{1 4}$ & $\mathbf{1 6}$ & 18 & 20 & $\ldots$ & $2 \mathrm{a}+8$ \\
& $\mathbf{1 4}$ & $\mathbf{1 6}$ & 18 & 20 & 22 & 24 & $\ldots$ & $2 \mathrm{a}+12$ \\
\hline
\end{tabular}

When similar tables for the other $\mathrm{n}$ groups are made, and when an addition is done by order between selected $n$ pieces of the smallest consecutive x number group elements and elements of y groups that each group of $y$ contains $n$ pieces of consecutive odd numbers, the result will be like in the tables. Here, the first group of $y$ is the same with x column. The first and the smallest number of the next y group is greater by 2 than the first and the smallest number of the previous y group. The sum of $b$ is always an even number as well.

As the first y group is also $\mathrm{x}$ group and as also the biggest number of $\mathrm{x}$ group is always $2 \mathrm{n}+1$, the biggest number of first b group must always become $2(2 n+1)=4 n+2$. This number is the first number which starts to be common with all the other $\mathrm{b}$ even numbers which are formed by different $\mathrm{x}$ elements for the same $n$ value in the tables; because it is in the last emerging line. Each even number after this number can be formed absolutely as $n$ pieces the number itself is included as well. As 1 piece of selected consecutive $n$ pieces odd numbers must be prime number until $n+1$ group, minimum 1 piece of the even numbers which are in each even number group has $n$ pieces of the same even number must be sum of two prime numbers.

Here, if also $4 n+4$ number after $4 n+2$ number is included, if all of emerging $n$ pieces of the same $4 n+4$ even numbers are not the numbers of $n+1$ group in a table for the same $n$ value, as all results of $4 n+4$ and $4 n+2$ form set of even numbers greater than 8 , then it means each even number which is greater than 8 absolutely must be sum of two prime numbers.

The equation between a values that first a namely $a_{1}$ which gives even numbers in the first line for $\mathrm{a}=[1, \infty)$ over $2 \mathrm{a}+4$, and $\mathrm{a}_{\mathrm{n}}$ which accepts the first even as $4 \mathrm{n}+2$ and gives the numbers of $2 \mathrm{a}+4 \mathrm{n}$ is (273)

$$
\mathrm{a}_{1}=\mathrm{a}_{\mathrm{n}}+2 \mathrm{n}-2
$$

Here, result of $a_{1}$ for $a_{n}=1$ is also equal to number of used odd numbers in the first y line to form $4 \mathrm{n}+2$ number or is equal to number of used numbers which are different than each other in the tables for the same $n$ value, outside of using $a_{n}$ to form $2 a_{n}+4 n$ namely $4 n+2$ number; because it is also $\mathrm{x}+\mathrm{y}$ operation number of forming $4 \mathrm{n}+2$ even number in the first line; therefore $a_{1}$ number over (273) for $a_{n}=2$ is required to form $4 n+4$, and the equation becomes (274), and states, that there are $2 \mathrm{n}$ times odd numbers on first $\mathrm{y}$ line.

$$
\mathrm{N}=2 \mathrm{n}
$$

\subsubsection{The result}

As the result for the above stated information, assume that $4 n+2$ and $4 n+4$ numbers cannot be sum of two prime numbers for each $n$ value. Last $n$ pieces of y consecutive odd numbers which are required to form $4 n+2$ and $4 n+4$ numbers for required $n$ value, must be $n$ group numbers; thus it means only $2 n-n=n$ pieces y consecutive odd numbers can be used, being 3 is the first usable number on the first $y$ line; but already this means, that all numbers except 1 are non-prime consecutive odd numbers for each $n$ value that is impossible. Even if it is assumed, that for the worst possibility, $n$ and $n-1$ groups emerge together by the same numbers as non-unique groups, also the information stated above says this assumption is impossible. Already if it is impossible even for $n$ group, when it is assumed that there are another groups,

$$
2 \mathrm{n}-\sum_{\mathrm{n}=2}^{\mathrm{n}} \mathrm{n}
$$

number of used or usable consecutive y odd numbers will decrease by (275), and is impossible for $n>1$ definition.

As and if we do not know prime separation, for the worst possibility of number of existent primes in $2 \mathrm{n}$ pieces usable consecutive odd numbers on the first y line, assume that for $n$, there are consecutive unique $n$ groups until last $2 n$ number even if actually they cannot be fitted such that as emerging number of the numbers will be bigger than $2 \mathrm{n}$ by this way; but if it is right, then amount will not be important that you can assume that there are $(2 n)^{2}$ pieces $n$ groups if essence of the function provides this that it is provided here; because the number of the non-prime numbers will increase greater than the primes for assumed $\mathrm{n}$ pieces unique groups since prime number is going to be fixed even for increasing non-prime odd numbers; so as unique groups are between two primes, there must be $2 \mathrm{n}$ pieces prime numbers. As this $2 \mathrm{n}$ is also equal to number of the used numbers on the first $y$ line that it is not important which numbers of $2 \mathrm{n}$ pieces numbers are prime or not prime here, absolutely minimum 1 piece of each $n$ pieces the same even $4 n+2$ numbers and minimum 1 piece of each $n$ pieces the same even $4 n+4$ numbers which emerge in each table separately are absolutely sum of two prime numbers. Also it means that all even numbers greater than 8 are sum of two prime numbers. This is also proof of infinite number of twin primes.

\subsection{Twin primes}

Select any unique number group which has $\mathrm{n}$ pieces of consecutive non-prime odd numbers. This group has to exist between 2 prime numbers according to the definition stated at the beginning between prime numbers and group numbers; because otherwise there will occur a group like $n+1$ group instead of $n$ group that actually infinite number of non-prime numbers can be consecutive. For example, let us take consecutive multiples of $3,5,7,9$ and 11 for $n=5$ group. Being 
a is an odd number, any multiples of odd numbers become $\mathrm{a}(2 \mathrm{x}+1)$ for required $\mathrm{x}$; so over $\left(6 \mathrm{x}_{1}+3\right)+2=10 \mathrm{x}_{2}+5$, it becomes $\mathrm{x}_{1}=5 \mathrm{x}$. Over $\left(6 \mathrm{x}_{1}+3\right)+4=14 \mathrm{x}_{3}+7$, it becomes $\mathrm{x}_{1}=7 \mathrm{x}$. Over $\left(6 \mathrm{x}_{1}+3\right)+6=18 \mathrm{x}_{4}+9$, it becomes $\mathrm{x}_{1}=9 \mathrm{x}$. Over $\left(6 \mathrm{x}_{1}+3\right)+8=22 \mathrm{x}_{5}+11$, it becomes $\mathrm{x}_{1}=11 \mathrm{x}$. Results of $6 \mathrm{x}_{1}+3$ which are odd multiples of 3 become $30 \mathrm{x}+3$, $42 \mathrm{x}+3,54 \mathrm{x}+3$ and $66 \mathrm{x}+3$ for the stated $\mathrm{x}_{1}$ values. If also these are made equal to each other, being $(11 \cdot 9 \cdot 7 \cdot 5 \cdot \mathrm{x})=\mathrm{m}$ and the first number of the group is multiple of 3 , consecutive multiples of the group numbers become by order $6 \mathrm{~m}+3$, $6 \mathrm{~m}+5,6 \mathrm{~m}+7,6 \mathrm{~m}+9$ and $6 \mathrm{~m}+11$. For more consecutive odd multiples, we can increase the number of used numbers in a group forever.

I selected $n_{1}, n_{2}, n_{3}$ and $n_{4}$ consecutive odd numbers in $\mathrm{n}=4$ group like $\mathrm{p}_{1} \mathrm{n}_{1} \mathrm{n}_{2} \mathrm{n}_{3} \mathrm{n}_{4} \mathrm{p}_{2}$ being $\mathrm{p}$ is prime number. Minimum one of these $n$ numbers has to be multiple of 3 ; because separation of odd multiples of 3 is according to $6 x+3$, and so there are always 2 consecutive odd numbers between two consecutive odd multiples of 3 . Here, if $n_{2}$ becomes odd multiple of 3 , then $\mathrm{p}_{2}$ must be the next multiple of 3 that this is only possible for $\mathrm{n}=5$. If $\mathrm{n}_{3}$ becomes odd multiple of 3 , then $\mathrm{p}_{1}$ must be the previous multiple of 3 that this is also possible for $n=5$. As it was said, it is possible to form groups have infinite number of consecutive non-prime odd number, namely $\mathrm{n}=4$ must exist anyway.

If $n_{1}$ becomes odd multiple of 3 , then $n_{4}$ must be the next multiple of 3 and also $n_{5}$ becomes the next multiple of 3 after $n_{4}$ as $n_{0}$ became the previous odd multiple of 3 before $n_{1}$ over $\mathrm{n}_{0} \mathrm{n}_{\mathrm{x}} \mathrm{p}_{1} \mathrm{n}_{1} \mathrm{n}_{2} \mathrm{n}_{3} \mathrm{n}_{4} \mathrm{p}_{2} \mathrm{n}_{\mathrm{y}} \mathrm{n}_{5}$.

If $n_{4}$ becomes odd multiple of 3 , then $n_{5}$ becomes the next multiple, and $n_{0}$ and $n_{1}$ become the previous multiples of 3; thus $n_{1}$ and $n_{4}$ are pretty suitable to be odd multiple of 3 .

Here, infinite number off odd consecutive $n$ number can take place after $n_{5}$; so element number of the next group after $\mathrm{n}=4$ is not important; but $\mathrm{n}_{\mathrm{y}}$ is always prime or not, this is important. Over $\mathrm{n}_{\mathrm{y}}=\mathrm{n}_{5}-2=(6 \mathrm{x}+3)-2$, it becomes $\mathrm{n}_{\mathrm{y}}=6 \mathrm{x}+1$. Hence, $\mathrm{n}_{\mathrm{y}}$ never can be only prime number where $x \in \mathbb{Z}^{+} \wedge x>0$. It is not prime for required $x$, and otherwise it is prime for emerging odd numbers between two $\mathrm{n}_{\mathrm{y}}$ and $\mathrm{n}_{\mathrm{y}+1}$ numbers which are a result of consecutive two $\mathrm{x}$ and $\mathrm{x}+1$ values; so when it becomes $\mathrm{n}_{\mathrm{y}}=\mathrm{p}_{3}$, it is a twin prime group between $n_{4}$ and $n_{5}$; thus twin primes are in infinite number.

\subsection{Collatz Problem}

The main question about the Collatz Problem is also pretty clear. When a positive whole number is selected, if the number is an even number then it is divided by 2 ; otherwise it is multiplied by 3 , and after that 1 is added to the result. When the same operation with required option of the problem due to the condition of to be odd or even number of the result is repeated for the last results, can each positive integer which is different than 0 and 1 be reduced into 1 ?

\subsubsection{The solution}

If the input number is an even number, and if it is not an even number as $2^{n}$ as well for definition of $n \in \mathbb{Z}^{+} \wedge n>0$; being pn is process number, when the input number is divided by pn times 2 or directly by $2^{\mathrm{pn}}$, each positive even number absolutely turns into a positive odd number as they can be defined as $(2 \mathrm{x}+1) \cdot 2^{\mathrm{n}}$ for definition of $\mathrm{x}, \mathrm{n} \in \mathbb{Z}^{+} \wedge \mathrm{n}>$ $1 \wedge x>0$; thus we should only work over odd numbers.

$$
\mathrm{a}_{\mathrm{n}+1}=\frac{3 \mathrm{a}_{\mathrm{n}}+1}{2}
$$

Over (276), it must become $\mathrm{a}_{\mathrm{n}+1}=\{2,5,8,11, \ldots ., 3 \mathrm{x}-$ 1\} where $x \in \mathbb{Z}^{+} \wedge x>0$ for a limited interval. For the numbers which make $\mathrm{a}_{\mathrm{n}}$ an odd number, it becomes $\mathrm{a}_{\mathrm{n}+1}=$ $\{5,11,17, \ldots, 6 \mathrm{x}-1\}$ for a limited interval and the same $\mathrm{x}$ definition. Also it becomes $\mathrm{a}_{\mathrm{n}}=\{3,7,11,15, \ldots ., 4 \mathrm{x}-1\}$ over $a_{n+1}$ odd numbers for the same conditions.

The below is a table over $a_{n}$ and $a_{n+1}$ numbers by order over (276) for a limited interval being $\mathrm{E}$ is even and $\mathrm{O}$ is odd.

Table 9: $\mathrm{a}_{\mathrm{n}}$ and $\mathrm{a}_{\mathrm{n}+1}$ numbers

\begin{tabular}{l|lllllll}
\hline$a_{n}$ & 3 & 7 & 11 & 15 & 19 & 23 & $\ldots$ \\
$a_{n+1}$ & 5 & 11 & 17 & 23 & 29 & 35 & $\ldots$ \\
$a_{n+2}$ & $E$ & $O$ & $E$ & $O$ & $E$ & $O$ & $\ldots$ \\
\hline
\end{tabular}

On the table of 9 , for $12 \mathrm{x}-7$ numbers from $\mathrm{a}_{\mathrm{n}+1}$ numbers with the same x definition, it becomes (277).

$$
18 \mathrm{x}-10=\frac{3(12 \mathrm{x}-7)+1}{2}
$$

The result of (277) is absolutely even number for each $\mathrm{x}$. For $12 \mathrm{x}-1$ numbers from $\mathrm{a}_{\mathrm{n}+1}$ numbers with the same $\mathrm{x}$ definition, it becomes (278).

$$
18 \mathrm{x}-1=\frac{3(12 \mathrm{x}-1)+1}{2}
$$

The result of (278) is absolutely odd number for each $\mathrm{x}$. Right this point, the question is this that for (279),

$$
\mathrm{a}_{\mathrm{n}+2}=\frac{3 \mathrm{a}_{\mathrm{n}+1}+1}{2}
$$

when $\mathrm{a}_{\mathrm{n}+2}$ becomes an even number and divided by $2^{\mathrm{pn}}$, does emerging odd numbers as a result always emerge before $a_{n+1}$ in set of odd numbers due to number order or can it be bigger number than $a_{n+1}$ ?

As the answer, if the result of the operation of (279) becomes even, to realize of to be reduced of the result of $3 \mathrm{a}_{\mathrm{n}+1}+1$

$2 \cdot 2^{\text {pn }}$ operation into an odd number which is before $a_{n+1}$, the condition of (280) always has to be provided.

$$
1>\frac{\frac{3 a_{n+1}+1}{2 \cdot 2^{p n}}}{a_{n+1}}
$$

If (280) is edited then as (281),

$$
1>\frac{1}{2^{\text {pn+1 }}}\left(3+\frac{1}{a_{n+1}}\right)
$$

the inequality of (281) always provides this for the definition of pn $>0 \wedge a_{n+1}>1 \wedge$ pn, $a_{n+1} \in \mathbb{Z}^{+}$.

As a result, when $a_{n+2}$ is reduced into an odd number, the odd number is always before $\mathrm{a}_{\mathrm{n}+1}$ odd, and is smaller than it. It means that the odd number as a result of $\mathrm{a}_{\mathrm{m}}=\frac{3 \mathrm{a}_{\mathrm{n}+1}+1}{2 \cdot 2^{\mathrm{pn}}}$ is always smaller than $a_{n+1}$ number. Even if $\frac{3 a_{m}+1}{2}$ becomes even number, again it can be reduced into a smaller 
odd number than both $\mathrm{a}_{\mathrm{m}}$ and $\mathrm{a}_{\mathrm{n}+1}$, and it is acceptable for the other repeats as well.

Right this point, a second question emerges that is there a number which always gets bigger and does not become an odd number on (276) infinite chain.

As the answer, there is table for the numbers which do not emerge on table 9. If these numbers are included to the numbers on table 9 as well, the sum is set off odd numbers. There will no other odd number which is not included to the calculations.

Table 10: The numbers which are not in the previous table

\begin{tabular}{lllllllll}
\hline $\mathbf{5}$ & 9 & 13 & $\mathbf{1 7}$ & 21 & 25 & $\mathbf{2 9}$ & $\ldots$ & $4 \mathrm{x}+1$ \\
\hline
\end{tabular}

The numbers which are written thick are also in $a_{n+1}$ line in table 9. The other numbers are the numbers which are not in table 9 .

If some groups are made for $4 \mathrm{x}+1$ numbers in table 10 , there will only emerge 3 groups for $12 x-3,2 x+1$ and $12 x-7$ numbers for the same $\mathrm{x}$ definition. Being $\mathrm{a}_{\mathrm{n}+1}=12 \mathrm{x}-3$, (282) always gives even result.

$$
18 \mathrm{x}-8=\frac{3(12 \mathrm{x}-3)+1}{2}
$$

Being $\mathrm{a}_{\mathrm{n}+1}=12 \mathrm{x}+1,(283)$ always gives even number result as well.

$$
18 \mathrm{x}+2=\frac{3(12 \mathrm{x}+1)+1}{2}
$$

As $12 \mathrm{x}-7$ numbers, they are already the same numbers with $a_{n+1}$, and at the result of (279) they always give even number being $\mathrm{a}_{\mathrm{n}+1}=12 \mathrm{x}-7$.

As even numbers, they can always be reduced into a smaller odd number than the odd number which makes them even number in the operation of (276) as it was proved; thus if each one of $\mathrm{a}_{\mathrm{n}}=4 \mathrm{x}-1$ numbers do not get greater by turning into an odd number when (276) is repeated for each n number where $n \in \mathbb{Z}^{+}$, it means all positive whole numbers different than 0 and 1 can be reduced into 1 .

In table $9,8 x-5$ numbers from $a_{n}$ numbers turn into $a_{n+1}$ number which gives even result over (279); thus the only chance is to give odd result always of $8 x-1$ numbers over (276) infinite chain. To be realized of this, the same numbers with $a_{n}$ must emerge on $a_{n+1}$ line in table 9. Also always the numbers which give odd result must emerge on $a_{n+1}$ line between them. For the same $\mathrm{x}$ condition, $4 \mathrm{x}-1$ and $6 \mathrm{x}-1$ operations give $a_{n}$ and $a_{n+1}$ over (276) for the same $x$ number; so the waited loop occurs or does not occur,

$$
\mathrm{x}_{1}=\frac{6 \mathrm{x}_{2}}{4}
$$

(284) shows this over the equality of $4 \mathrm{x}_{1}-1=6 \mathrm{x}_{2}-1$. For (284), it becomes $\mathrm{x}_{1}=3 \mathrm{t}$ and $\mathrm{x}_{2}=2 \mathrm{t}$ over $\mathrm{t} \in \mathbb{Z}^{+} \wedge \mathrm{t}>0$ condition; so the problem is reduced into the rule of table 11 below.

Table 11: The numbers for each $t$

\begin{tabular}{l|lllllll}
\hline $\mathbf{2 t}$ & 2 & 4 & 6 & 8 & 10 & 12 & $\ldots$ \\
$\mathbf{3 t}$ & 3 & 6 & 9 & 12 & 15 & 18 & $\ldots$ \\
\hline
\end{tabular}

Each number on $4 \mathrm{x}-1$ and $6 \mathrm{x}-1$ is also order number of $a_{n}$ and $a_{n+1}$ in table 11; thus the number has $3 \mathrm{t}$ order number on $a_{n}$ or $2 t$ line and the number has $2 t$ order number on $a_{n+1}$ or $3 \mathrm{t}$ line in table 11 are the same numbers.

As the odd numbers on $3 \mathrm{t}$ line in table 11 are $\mathrm{a}_{\mathrm{n}+1}$ numbers which give even result in table 9, they are elected; thus table 11 turns into table 12 .

Table 12: The other numbers for each $t$

\begin{tabular}{l|lllllll}
\hline $\mathbf{4 t}$ & 4 & 8 & 12 & 16 & 20 & 24 & $\ldots$ \\
$\mathbf{6 t}$ & 6 & 12 & 18 & 24 & 130 & 36 & $\ldots$ \\
\hline
\end{tabular}

In table 12, to occur of the infinite loop, when a $4 \mathrm{t}$ number is selected, also 6t number which is under it and on $6 \mathrm{t}$ line in table 12 must be even number, and also this $6 \mathrm{t}$ number must take place on $4 \mathrm{t}$ line again. This condition has to take place for one or more than one number to be broken of the Collatz's reducing chain, and then one or more than one number will not be reduced into 1; but this is impossible; because for $t_{n+1}=\frac{6 t_{n}}{4}$ where $t>0 \wedge t, n \in \mathbb{Z}^{+}$, for each $t$ whole number,

$$
t_{n+1, t}=\lim _{n \rightarrow \infty} \frac{6 t_{n, t}}{4}
$$

(285) has to be provided for the condition of (286),

$$
\mathrm{t}_{\mathrm{n}, \mathrm{t}}, \mathrm{t}_{\mathrm{n}+1, \mathrm{t}} \in \mathbb{Z}^{+}
$$

where $t_{n, t}=4 t$, $t$ is the order number of the number which is waited of starting the loop from it, and $n$ is the repeat number of (285) for each t. As this condition of (286), it cannot be provided for each $t$. For example, for $t_{1,1}=4$, it becomes $6 \mathrm{t}_{1,1}=4 \mathrm{t}_{2,1}$ and so becomes $\mathrm{t}_{2,1}=6$. For $\mathrm{t}_{2,1}=6$, it becomes $6 \mathrm{t}_{2,1}=4 \mathrm{t}_{3,1}$ and so becomes $\mathrm{t}_{3,1}=9$. For $\mathrm{t}_{3,1}=$ 9 , it becomes $6 \mathrm{t}_{3,1}=4 \mathrm{t}_{4,1}$ and so becomes $\mathrm{t}_{4,1}=27 / 2$. As it can be seen, $t_{4,1} \notin \mathbb{Z}^{+}$and so the condition of (286) cannot be provided.

\subsubsection{The result}

(285) cannot continue forever; because wee need a number which has infinite number of common divisors like $4^{\infty}$ or $(2 \mathrm{x}+1) \cdot 4^{\infty}$ imaginary numbers. As it can be seen, only we can increase the repeat number by using a t number like $4^{\mathrm{m}}$ where $\mathrm{m}>0 \wedge \mathrm{m} \in \mathbb{Z}^{+}$that if $\mathrm{m}$ gets bigger, then the repeat will increase; but there is no infinite repeat; hence, any whole number absolutely can be reduced into 1 by changing operation numbers of the Collatz's rule due to the used number.

For the repeats of (276), being $2^{\mathrm{m}}$ is order number of the selected odd number of $a_{n}$ in table 9 where $m>0 \wedge m \in \mathbb{Z}^{+}$, it gives $m+2$ pieces odd number, and then the last one gives even number on (276) for $2^{\mathrm{m}}$. Table 13 is a demonstration of this.

Table 13: The other numbers for each $t$

\begin{tabular}{l|lllll}
\hline $\mathbf{m}$ & & & & $\mathbf{a}_{\mathbf{n}}$ & \\
\hline $\mathbf{1}$ & $7 \rightarrow$ & $11 \rightarrow$ & 17 & & \\
$\mathbf{2}$ & $15 \rightarrow$ & $23 \rightarrow$ & $35 \rightarrow$ & 53 & \\
$\mathbf{3}$ & $31 \rightarrow$ & $47 \rightarrow$ & $71 \rightarrow$ & $107 \rightarrow$ & 161 \\
$\boldsymbol{\bullet}$ & & & & & \\
$\mathbf{\cdot}$ & & & & & \\
\hline
\end{tabular}


Also we can use an operation like (287), and we can derive another operation as well. The below is an example.

$$
\mathrm{a}=7+\sum_{\mathrm{m}=3}^{\mathrm{m}} 2^{\mathrm{m}}
$$

Here for (287), $\mathrm{m}+1$ pieces odd numbers or repeats on (276) emerges being $a=a_{0}$ which is first input number on (276). You can write your own operation as well.

\subsection{Perfect square of $\mathbf{p}-1$}

Are there infinitely many primes $\mathrm{p}$ such that $\mathrm{p}-1$ is a perfect square?

\section{Argument}

Here, $p-1$ is an even number where $\left\{p \in \mathbb{Z}^{+}\right\}$. If it is turned into $\mathrm{e}^{2}+1=\mathrm{p}$ where $\mathrm{e}$ is an even number. As prime numbers are odd numbers, they can only exist between two consecutive odd multiples of 3 . As there are only two places in-between for odd numbers, for the previous position relatively to multiples of 3 , the equation becomes $\mathrm{e}^{2}+1=$ $6 \mathrm{x}+1$, and finally turns into Eq. (288).

$$
\mathrm{x}=\frac{\mathrm{e}^{2}}{6}
$$

Right this point, let e to be a number like $6 \mathrm{a}$. For this condition, $x$ directly shall become $6 \mathrm{a}^{2}$; so for Eq. (288a),

$$
\mathrm{e}^{2}+1=36 \mathrm{a}^{2}+1
$$

it can be said that a second analysis is required that $\mathrm{p}=$ $36 \mathrm{a}^{2}+1$ equation can always be provided or not, where $\mathrm{p}$ is prime. Right this point, if it is assumed that numbers come from $-\infty$ direction to $+\infty$ on number line, then for the previous odd numbers relatively to multiples of 3 , the destination equation of previous position of multiples of 3 which is $\mathrm{f}(\mathrm{x})=6 \mathrm{x}+1$ becomes $\mathrm{f}(\mathrm{r}, \mathrm{t})=6 \mathrm{r}-(6 \mathrm{t}-1)$ to start before 0 to $+\infty$ for each $\mathrm{r}$ and $\mathrm{t}$ positive integers; then for $36 \mathrm{a}^{2}+1=6 \mathrm{r}-(6 \mathrm{t}-1)$ equation, the equation becomes Eq. (288b).

$$
\mathrm{r}=6 \mathrm{a}^{2}+\mathrm{t}
$$

Hence, for each a positive integer, $\mathrm{e}^{2}+1=\mathrm{p}$ can be reduced to place each previous position before multiples of 3 . As all previous position cannot be non-prime number, forever it becomes prime number by some intervals.

As the result, it can be said that, as first degree place function is not possible for primes as it was proved the above, also no exponential function can be written as $\mathrm{f}(\mathrm{m})=\mathrm{m}^{\mathrm{n}}$; because $\mathrm{m}^{\mathrm{n}}$ never passes a place when intersection points for inbetween numbers are determined for any $f(r, t)=6 r-(6 t-1)$ which starts to give in-between numbers from negative direction of number line to $+\infty$. It always intersects.

\subsection{The Arc Side}

Here the emerging lengths like SR, GR or GS seem can take any value; but it is not true. They are dependent on change in radius, angle or circumference of the circle. We can find them out by relating with the other lengths if also they are known. You can catch other relation as well aside the above stated ones.

Warning
It is possible to compute trigonometric functions and $\pi$ like calculating a root of an integer. It can reduce process number of power series.

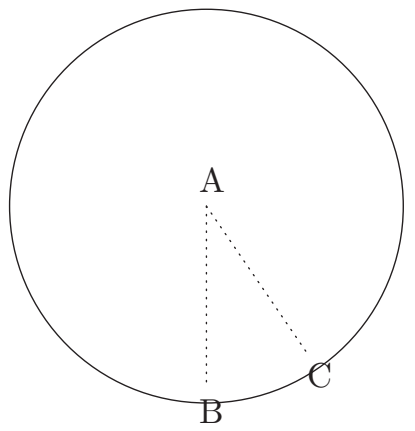

Fig. 45: Two points on the circumference

As the first step, let choosing two points like $\mathrm{B}$ and $\mathrm{C}$ on Fig. 45, and know that; $\angle \mathrm{BAC}$, the radius and so $\overparen{\mathrm{BC}^{\prime}}$ are known. The angle should not be more than $45^{\circ}$. The other angles between $45^{\circ}-90^{\circ}$ will be found out by using half angle formula of $\cos (2 \alpha)=\cos ^{2}(\alpha)-\sin ^{2}(\alpha)$. Otherwise its projection must be drawn for the other side of $\mathrm{AB}$.

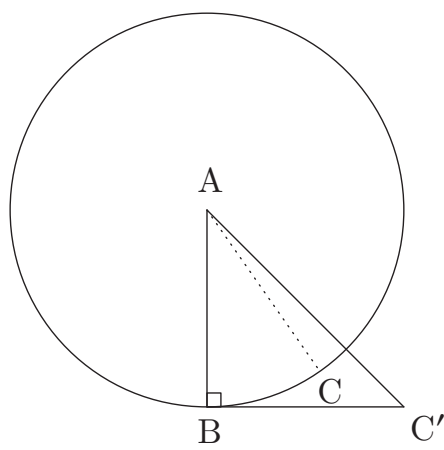

Fig. 46: The arc side

Thereupon as the second step, open $\overparen{\mathrm{BC}}$ as $\mathrm{BC}^{\prime}$ where the radius is the height as the same as on Fig. 46, and lengthen it as $2|\overparen{\mathrm{BC}}|$. Know that, as $|\overparen{\mathrm{BC}}|$ is known, also $\left|\mathrm{BC}^{\prime}\right|$ and so $\left|\mathrm{AC}^{\prime}\right|$ are known.

If a circle is drawn for $\mathrm{AC}^{\prime}$ radius, then it will look like on Fig. 47, and know that $\mathrm{C}$ point is not on EG. The equation becomes Eq. (289),

$$
|\mathrm{AD}|=\frac{|\mathrm{AE}|^{3}}{|\mathrm{AF}|^{2}}
$$

where $|\mathrm{AG}|=|\mathrm{AE}|^{2} /|\mathrm{AF}|$ in $\triangle \mathrm{AEF}$ and $|\mathrm{AD}|=|\mathrm{AG}|^{2} /|\mathrm{AE}|$ in $\triangle \mathrm{AGE}$.

It will be as Eq. (289a),

$$
|\mathrm{DG}|=\frac{|\mathrm{AB}| \cdot\left|\mathrm{BC}^{\prime}\right|}{\sqrt{|\mathrm{AB}|^{2}+\left|\mathrm{BC}^{\prime}\right|^{2}}}
$$

where $|\mathrm{AD}| /|\mathrm{AB}|=|\mathrm{DG}| /\left|\mathrm{BC}^{\prime}\right|$ from the similarity in $\triangle \mathrm{ABC}^{\prime},|\mathrm{AD}|=|\mathrm{AG}|^{2} /|\mathrm{AE}|$ by using Euclid relation in $\triangle \mathrm{AGE}$ and $|\mathrm{AE}|=\sqrt{|\mathrm{AB}|^{2}+\left|\mathrm{BC}^{\prime}\right|^{2}}$ equation for $|\mathrm{AB}|=$ $|\mathrm{AG}|$ and $|\mathrm{EG}|=\left|\mathrm{BC}^{\prime}\right|$. 


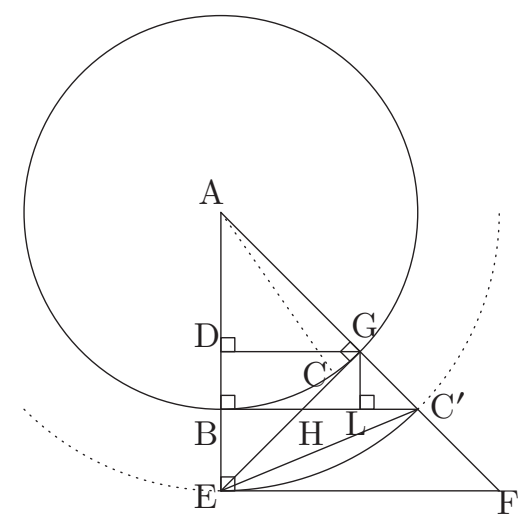

Fig. 47: The second radius

For Eq. (289), $|\mathrm{DB}|=|\mathrm{AB}|-|\mathrm{AD}|$ equation turns into Eq. (289b).

$$
|\mathrm{DB}|=|\mathrm{AB}|-\frac{|\mathrm{AE}|^{3}}{|\mathrm{AF}|^{2}}
$$

As the equations are $|\mathrm{DB}|=|\mathrm{GL}|,|\mathrm{GH}|=|\mathrm{BH}|$ and $|\mathrm{EH}|=\left|\mathrm{HC}^{\prime}\right|$, for $|\mathrm{GL}| /|\mathrm{BE}|=|\mathrm{GH}| /|\mathrm{HE}|$ it will be as $|\mathrm{DB}| /(|\mathrm{AE}|-|\mathrm{AF}|)=|\mathrm{GH}| /\left(\left|\mathrm{BC}^{\prime}\right|-|\mathrm{GH}|\right)$, and when it is edited, it turns into Eq. (289c).

$$
|\mathrm{GH}|=\frac{\left|\mathrm{BC}^{\prime}\right| \cdot|\mathrm{DB}|}{|\mathrm{AE}|-|\mathrm{AD}|}
$$

As $\triangle \mathrm{EBH}$ and $\triangle \mathrm{HLG}$ are similar triangles, the relation will be $|\mathrm{GL}| /|\mathrm{BE}|=|\mathrm{HG}| /|\mathrm{HE}|$, and it turns into Eq. (289d) for $|\mathrm{GL}|=|\mathrm{DB}|$ and $|\mathrm{HG}|=|\mathrm{BH}|$ equations.

$$
|\mathrm{HE}|=\frac{|\mathrm{HG}| \cdot|\mathrm{BE}|}{|\mathrm{DB}|}
$$

Here, Eq. (289d) is also $\left|\mathrm{HC}^{\prime}\right|$ for $\left|\mathrm{HC}^{\prime}\right|=|\mathrm{HE}|$ equation. As the equation is $|\mathrm{AE}|=\left|\mathrm{AC}^{\prime}\right|$, then it will be $|\mathrm{AE}|=$ $\sqrt{|\mathrm{AB}|^{2}+\left|\mathrm{BC}^{\prime}\right|^{2}}$, and finally turns into Eq. (289e).

$$
|\mathrm{BE}|=\sqrt{|\mathrm{AB}|^{2}+\left|\mathrm{BC}^{\prime}\right|^{2}}-|\mathrm{AB}|
$$

As we know $|\mathrm{HG}|$ as Eq. (289c), $|\mathrm{DB}|$ as Eq. (289b), and $|\mathrm{BE}|$ as Eq. (289e), then also we can find out Eq. (289d) after this.

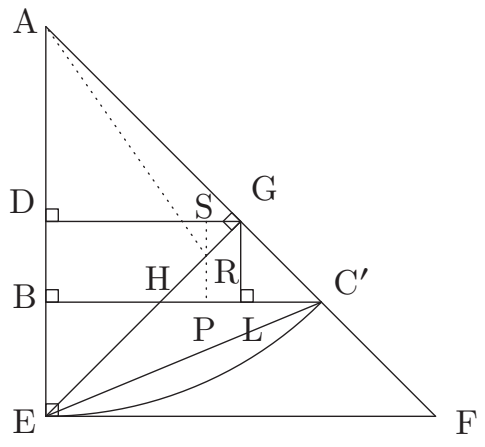

Fig. 48: A part of Fig. 47

If $\mathrm{AC}$ radius is intersected with $\mathrm{EG}$ by lengthening, and just a part is taken from Fig. 47, it will be as Fig. 48. As $\triangle \mathrm{GSR}$ and $\triangle \mathrm{GDE}$ are similar triangles, it will be Eq. (289f).

$$
\frac{|\mathrm{GS}|}{|\mathrm{GD}|}=\frac{|\mathrm{SR}|}{|\mathrm{DE}|}
$$

In $\triangle \mathrm{GSR}$, as it will be $|\mathrm{GR}|=\sqrt{|\mathrm{SR}|^{2}+|\mathrm{GS}|^{2}}$ also it will be Eq. (289g) over Eq. (289f) or over the similarity between $\Delta \mathrm{GDE}$ and $\Delta \mathrm{GSR}$ where $|\mathrm{GE}|=\sqrt{|\mathrm{GD}|^{2}+|\mathrm{DE}|^{2}}$.

$$
|\mathrm{GR}|=\frac{|\mathrm{SR}| \sqrt{|\mathrm{GD}|^{2}+|\mathrm{DE}|^{2}}}{|\mathrm{DE}|}
$$

For $(|\mathrm{DB}|-|\mathrm{SR}|) /|\mathrm{BE}|=|\mathrm{HR}| /|\mathrm{EH}|$ equation over the similarity between $\triangle \mathrm{RPH}$ and $\triangle \mathrm{HBE},|\mathrm{HG}|=|\mathrm{GR}|+|\mathrm{HR}|$ equation turns into Eq. (289h) for $|\mathrm{SR}|$.

$$
|\mathrm{SR}|=\frac{|\mathrm{DE}|(|\mathrm{HG}| \cdot|\mathrm{BE}|-|\mathrm{EH}| \cdot|\mathrm{DB}|)}{|\mathrm{BE}| \sqrt{|\mathrm{GD}|^{2}+|\mathrm{DE}|^{2}}-|\mathrm{DE}| \cdot|\mathrm{EH}|}
$$

If Eq. (289h) is used on Eq. (289f), it will be as Eq. (289i).

$$
|\mathrm{GS}|=\frac{|\mathrm{GD}|(|\mathrm{HG}| \cdot|\mathrm{BE}|-|\mathrm{EH}| \cdot|\mathrm{DB}|)}{|\mathrm{BE}| \sqrt{|\mathrm{GD}|^{2}+|\mathrm{DE}|^{2}}-|\mathrm{DE}| \cdot|\mathrm{EH}|}
$$

Being the equations are as $|\mathrm{GR}|=\sqrt{|\mathrm{SR}|^{2}+|\mathrm{GS}|^{2}}$, $|\mathrm{ER}|=|\mathrm{EG}|-|\mathrm{GR}|,|\mathrm{AR}|=\sqrt{|\mathrm{AG}|^{2}+|\mathrm{GR}|^{2}},|\mathrm{BC}|=|\mathrm{EG}|$ over the law of cosines for $\angle \mathrm{EAR}=\alpha$, it will be as Eq. (290).

$$
\cos \alpha=\frac{|\mathrm{AE}|^{2}+|\mathrm{AG}|^{2}-\left|\mathrm{BC}^{\prime}\right|^{2}+2\left|\mathrm{BC} \mathrm{C}^{\prime}\right| \cdot|\mathrm{GR}|}{2|\mathrm{AE}| \sqrt{|\mathrm{AG}|^{2}+|\mathrm{GR}|^{2}}}
$$

\subsubsection{Area and length relations}

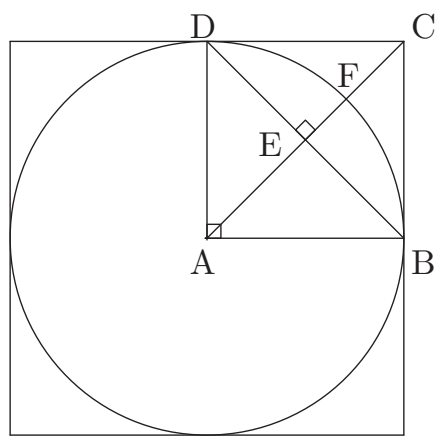

Fig. 49: Internal tangent circle

If an internal tangent circle is drawn in a square as the same as on Fig. 49, and if length of the square is changed forever gradually, then all the lengths of the circle in the square have to change in the same ratio; because $\mathrm{AD}$ is radius of the circle and also $|\mathrm{FC}|$ is related with the same radius $\mathrm{AF}$; so also all emerging lengths of the circle change at the same amount that; $|\mathrm{EF}|,|\mathrm{AE}|,|\mathrm{DE}|$ and $|\mathrm{EB}|$ are included as well naturally.

For example, as it is $|\mathrm{FC}|=|\mathrm{AB}| \sqrt{2}-|\mathrm{AB}|$, 


$$
\mathrm{n}=\frac{\mathrm{n}|\mathrm{AB}| \sqrt{2}-\mathrm{n}|\mathrm{AB}|}{|\mathrm{AB}| \sqrt{2}-|\mathrm{AB}|}=\frac{\left|\mathrm{FC}_{2}\right|}{|\mathrm{FC}|}
$$

for lengthened $|\mathrm{FC}|$ as $\left|\mathrm{FC}_{2}\right|=\mathrm{n}$, it becomes Eq. (291). It is always constant.

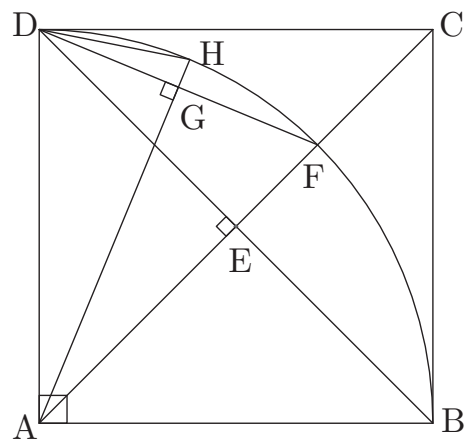

Fig. 50: The quarter slice of Fig. 49

During these changings, also ratios of the area which are between $\overparen{\mathrm{DB}}$ and $\mathrm{DB}$ chord are always conserved, any area in ABCD square is included as well; because if some triangles are drawn as dependent of the rule in Fig. 50 gradually forever, then there is going to always emerge some triangles with right angle instead of the circle slice; so for example, being $\mathrm{A}(\mathrm{BAD})=\mathrm{A}$,

$$
\mathrm{n}^{2}=\frac{2 \mathrm{n}^{2}|\mathrm{AB} \| \mathrm{AD}|}{2|\mathrm{AB} \| \mathrm{AD}|}=\frac{\mathrm{A}_{2}}{\mathrm{~A}}
$$

it becomes Eq. (292) and thus becomes $\mathrm{n}^{2}$ as constant for triangles where $\mathrm{A}_{2}$ is expanded area; therefore the area between $\overparen{\mathrm{DB}}$ and $\mathrm{DB}$ chord, and $\overparen{\mathrm{DB}}$ and $|\mathrm{DC}|+|\mathrm{CB}|$ also always change in the same rate.

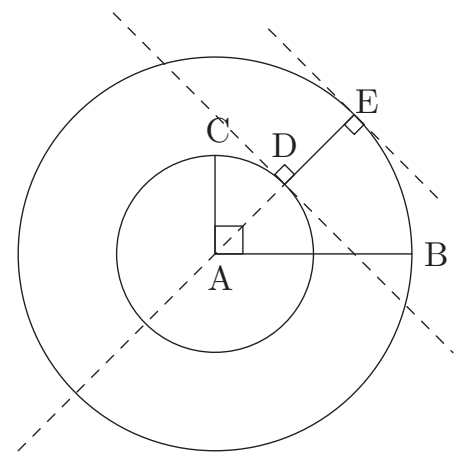

Fig. 51: The area scanned

Already it can be analyzed by looking at the radius, $\triangle \mathrm{ABD}$ and the other chords, the arcs are included as well. It can be said that ratio of two length which are intersected or independent of each other is the same and fixed for changing radius or square sides; so also ratio of the areas which are dependent of the lengths is the same and fixed as well, where lengths and areas are amongst themselves. If this is the condition, then for the circle area $\mathrm{A}, \mathrm{r}$ radius and square area $4 \mathrm{r}^{2}$ which emerges for the same radius, it is going to be a constant like $\mathrm{W}=4 \mathrm{r}^{2} / \mathrm{A}$. Thereupon, let the circle's area be a number like $\pi$ where $\mathrm{r}=1$ unit. For this radius, the equation becomes $4 \mathrm{r}^{2} / \mathrm{A}=4 / \pi$ over the $\mathrm{W}$ constant. Finally for changing $\mathrm{r}$ values, area of a circle becomes Eq. (293).

$$
\mathrm{A}=\pi \mathrm{r}^{2}
$$

On Fig. 51, there are two circles which were drawn nested manner, and have $|\mathrm{AC}|=\mathrm{r}_{1}$ and $|\mathrm{AB}|=\mathrm{r}_{2}$ radiuses. At the end of the scanning for 1 round, the area scanned by $|\mathrm{DE}|$ becomes $A_{2}-A_{1}$ for $|A C|=r_{1}$ and $|A B|=r_{2}$ radiuses. During the process, the distance taken by $\mathrm{E}$ point becomes $\mathrm{C}_{2}$, and by $\mathrm{D}$ point becomes $\mathrm{C}_{1}$. For $\mathrm{DE}$. The distance taken by infinite points between $\mathrm{D}$ and $\mathrm{E}$ points on $|\mathrm{DE}|$ becomes $\mathrm{A}_{2}-\mathrm{A}_{1}$ as also it was derived.

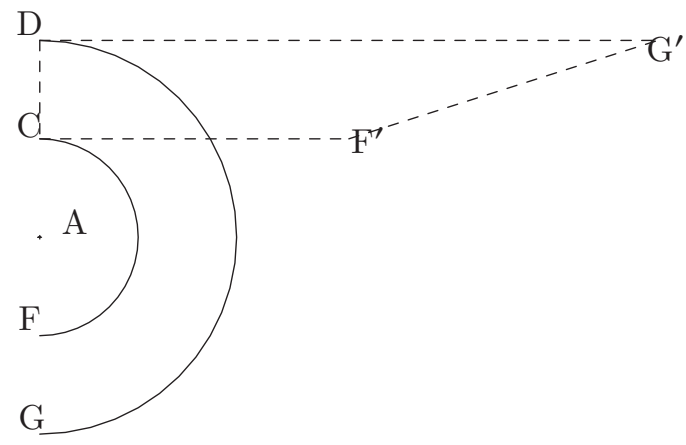

Fig. 52: The area of the circle in the kind of triangle

This process can be shown as Fig. 52 for the same radiuses even for half of the circle. It becomes $\left|\mathrm{DG}^{\prime}\right|=|\overparen{\mathrm{DG}}|=\mathrm{C}_{2} / 2$ and $\left|\mathrm{CF}^{\prime}\right|=|\overparen{\mathrm{CF}}|=\mathrm{C}_{1} / 2$. The area of the trapezoid becomes $\mathrm{A}\left(\mathrm{DG}^{\prime} \mathrm{F}^{\prime} \mathrm{C}\right)=\left(\mathrm{A}_{2}-\mathrm{A}_{1}\right) / 2$. While $\mathrm{r}_{1}$ radius is approaching to 0 , the area of trapezoid approaches to $\triangle \mathrm{DG}^{\prime} \mathrm{A}$, and also the area is approaching to a single circle's area with $\mathrm{r}_{2}$ radius. For the whole circle, it becomes Eq. (293a).

$$
\mathrm{A}=\lim _{\mathrm{r}_{1} \rightarrow 0} \pi\left(\mathrm{r}_{2}-\mathrm{r}_{1}\right)^{2}
$$

Hence it becomes $\pi \mathrm{r}^{2}$ for $\mathrm{r}_{2}=\mathrm{r}$ as there is only 1 radius after this. For this condition, after this area of the triangle becomes $\mathrm{Cr} / 2$ where $\mathrm{C}_{2}=\mathrm{C}$ for the whole circle. Finally over $\mathrm{Cr} / 2=\pi \mathrm{r}^{2}$ equation, circumference of a circle becomes Eq. (293b).

$$
\mathrm{C}=2 \pi \mathrm{r}
$$

\subsubsection{Computation of pi}

Relation between area and circumference of a circle is Eq. (294)

$$
\mathrm{C}=2 \mathrm{~A} / \mathrm{r}
$$

where $\mathrm{A}$ is area and $\mathrm{C}$ is circumference. If the area is an integer and the angle is an angle like $60^{\circ}$ which has a certain value for $\cos (60)$ which can be found out by using an equilateral triangle, then by using Eq. (290), r can easily be found out as the circumference is $\mathrm{A} / 3 \mathrm{r}$ and the radius is $\mathrm{r}$, by solving equation. By this way, also $\pi$ can be found out by infinite number of different integer area values for Eq. (295)

$$
\pi=\mathrm{A} / \mathrm{r}^{2}
$$

As the radius after all, if the angle, and the arc which is depended on $2 \pi \mathrm{r} \alpha / 360$ are known on Eq. (290), then $\pi$ directly 
can be found out for $\cos (60)$ and $r=1$ as well by solving equation.

\section{9 $P=N P$ ? Problem}

All the below stated inferences are from the determined rules of Fig. 1 at the beginning of the paper. I did not add the inequalities and inferences again here. All of them are from impossibility of perpendicularity.

\subsubsection{Magic of number world}

Since in a conservative limited area no two lengths can be equal to each other, the numbers which are defined as $\mathrm{x}^{\mathrm{y}} \cdot \mathrm{z}$ where $\mathrm{x}, \mathrm{y}, \mathrm{z} \in \mathbb{N}^{+}$are not real numbers. These are imaginary numbers since are also formed by two the same numbers. Namely, the numbers like 4,8,12,16 and 25 are not real numbers. Even if they can be obtained by addition, multiplication is a process alone as a phenomenon which has different, peculiar and exact properties. You cannot use always addition and subtraction instead of multiplication and division to achieve the same number. Namely, you cannot realize verifying always that this means if a number cannot be obtained by multiplication, then it means also addition will be meaningless. This is very important especially for physics; because in real physical medium it has different meanings. For example fine-tuning problem may occur because of this. While we are using some series to calculate something, we may ignore something important.

Even if we eliminate some numbers from the number line, still there do not exist only prime numbers. If there were only prime numbers, it would mean that the equality is always $\mathrm{P}=\mathrm{NP}$ since there is no prime factors, and would mean that each length of a polygon or a shape in the universe would get different side lengths as prime numbers that means the universe would be formed only by primes. Even so, still each length of a polygon has different side lengths that some of them are exactly prime numbers.

\subsubsection{First solution to the $\mathbf{P}=\mathrm{NP}$ ? problem}

Over the above stated information, first of all we can stated that as it was said as the above, higher dimensional parts have different sizes. It means that to create a 3 dimensional cube, if you have a 2 dimensional square which has different side lengths that has A energy in real physical medium, you must use a different square which has B energy since has different side lengths in itself and has different lengths than the other one where $\mathrm{A}<\mathrm{B}$ or $\mathrm{A}>\mathrm{B}$. It means to create higher numbers or higher dimensions you must use more energy. You can say that higher dimensions can multiply by getting smaller in size forever as also can get bigger; but it does not differ since you can go from the reverse direction. It also means, if a number is formed by multiplication of different prime numbers, you must use more energy to find the prime factors even if you assumed that you know the places where the prime numbers are placed there. It can be maintained as the following one.

The state of $\mathrm{P}=\mathrm{NP}$ is only dream.

\section{Proposition X}

There is a special condition here that if you accept the twin which has more energy of higher dimensional polygon and thus numbers as smaller numbers, then an excessive work emerges. It means, the problems which are not dependent on a single polynomial as NP problems, can be solved easier; but it requires soothsaying since information is deterministic. Stated information proves this over the above stated rules of the triangle of Fig. 1.

\section{Second solution to the $\mathbf{P}=\mathrm{NP}$ ? problem}

An additional supportive solution can be realized as well. In the set of odd numbers, assume that there are prime numbers where odd multiples of 3 are placed instead of odd multiples of 3 . In this case, also assume that the numbers inbetween are non-prime numbers.

$$
\mathrm{N}_{\mathrm{A} 1}\left|\mathrm{~N}_{\mathrm{B} 1}\right| \mathbf{9}\left|\mathrm{N}_{\mathrm{A} 2}\right| \mathrm{N}_{\mathrm{B} 2}|\mathbf{1 5}| \mathrm{N}_{\mathrm{A} 3}\left|\mathrm{~N}_{\mathrm{B} 3}\right| \mathbf{2 1}
$$

Table 14: Distribution of odd multiples of 3 for a limited interval. Here $\mathrm{N}_{\mathrm{A}}$ numbers are different non-prime numbers which occur after odd multiples of 3 , and $\mathrm{N}_{\mathrm{B}}$ numbers are different non-prime numbers occur before odd multiples of 3 since there are only 2 odd numbers between each consecutive odd multiples of 3 in the set of odd numbers.

Here is a table which was created over this assumption. Here we must ask that in the real set of odd numbers which is independent of the table, for a selected odd multiple of 3, are there equal number of prime numbers to the number of odd numbers between 3 and the selected odd multiple of 3 . If the answer is yes, then in the set of odd numbers between two consecutive odd multiple of 3 , there must exist minimum 1 prime number; but this is impossible since there always must be non-prime twins. For example even if we can choose infinite different combination, we can choose odd multiples of 5 and 7 as non-prime twins. Distribution of 5 in the set of odd numbers is over $f(x)=10 x-5$ function as 7 is over $f(y)=14 y-7$ as well; so as the difference between two consecutive multiple of 5 and 7 as twin is going to be 2 , the equation must be $f(x)=f(y)+2$. Over this equation it must be (296) where $\mathrm{x}, \mathrm{y} \in \mathbb{N}^{+}$

$$
5 \mathrm{x}=7 \mathrm{y}
$$

Here for each $\mathrm{x}$ and $\mathrm{y}$ values which provides the equality, there are infinite number of non-prime twins. Examples can be applied for any numbers like this equation. This is not only valid for 5 and 7.

This means that for a selected odd multiple of 3 there is not exactly 1 prime number between each consecutive odd multiples of 3 until the selected odd number. Namely number of non-prime numbers are more than prime numbers until the selected odd multiple of 3 . In this case, if we assume that there exist prime numbers in the places which odd multiples of 3 exist instead of odd multiples of 3 , then this means for the selected odd multiple of 3, non-prime numbers are going to be more than prime numbers due to the table. This increases possibility of to be non-prime number of a selected number in the set of odd number over the assumption and thus also increases prime factor processes since also more non-primes exist due to the assumption for a selected number. Numbers are formed by more non-prime numbers as unusual as also number of prime numbers are decreased by this assumption. This is for the worst possibility. Namely if we prove over this assumption that for a selected number, the process number for primality test is not equal to the process number for finding out prime factors, no other proofs are required since is the worst possibility.

In this case, over the above stated information now the function which gives each prime is known as $f(x)=6 x-3$ function since the function is distribution rule of odd multiples of 3 as primes are counted as exist at the places of these multiples as well.

As a result there is a function which test primality as (297) over $f(x)=6 x-3$.

$$
\mathrm{f}^{-1}(\mathrm{x})=\frac{\mathrm{x}+3}{6}
$$


If result is a whole number, then the number is prime, otherwise is not prime. If is not prime, then minimum $\left\lfloor\frac{x+3}{6}\right\rfloor$ process number requires that it means always for primality test of a number, less process requires than finding out prime factors.

For the worst possibility, the state of $\mathrm{P}=\mathrm{NP}$ is completely dream.

\section{Proposition Y}

As a result, $\mathrm{P}=\mathrm{NP}$ state is not possible that only 1 evidence is enough since assumed function which provides $\mathrm{P}=\mathrm{NP}$ state must also work for this primality and prime factor problem. Even if the above stated solution is not going to work for some numbers that especially at the beginning of the set of odd numbers, even only 1 number in the set of odd number can be counted as evidence. We must accept the set of odd numbers as infinite not in an interval. Already, in the infinite set, logically non-primes can be accepted as more than primes since when a new prime emerges, it is combined with the early emerging ones to create more number. This creates many possibilities and thus creates many combinations.

\section{Acknowledgements}

I shall not demand patent right. Anybody who wants to use the above stated things can use freely without asking.

Thanks to overleaf.com for the text editor and to online widgets of Wolfram Alpha for mathematical calculations.

\section{References}

1. Kavak M. On the Uncertainty Principle. American Journal of Physics and Applications, 2016, v.4(4), 90-123. Available online: https://osf.io/t8zqw/

2. Heisenberg W. Über den anschaulichen Inhalt der quantentheoretischen Kinematik und Mechanik. Zeitschrift für Physik, 1927, v. 43 (3), 172198.

3. Podolsky B, Einstein A, Rosen N,. Can Quantum-Mechanical Description of Physical Reality Be Considered Complete?. Physical Review, 1935, v. 47 (10), 777-780.

4. Lorentz H. De relatieve beweging van de aarde en den aether. Zittingsverlag Akad, 1892, p. 74

5. Michelson A, Morley E. On the Relative Motion of the Earth and the Luminiferous Ether. American Journal of Science, 1887, v. 34 (203), 333-345.

6. Planck M. On the Law of Distribution of Energy in the Normal Spectrum. Annalen der Physik, 1901, v. 4 (10), p. 553.

7. Yang C, Mills L. Conservation of isotopic spin and isotopic gauge invariance. Physical Review, 1954, v. 96, 191-195. 